University of Tennessee Health Science Center

UTHSC Digital Commons

\title{
Multiscale Genomic Analysis of the Corticolimbic System: Uncovering the Molecular and Anatomic Substrates of Anxiety- Related Behavior
}

Khyobeni Mozhui

University of Tennessee Health Science Center

Follow this and additional works at: https://dc.uthsc.edu/dissertations

Part of the Mental and Social Health Commons, Nervous System Commons, and the Neurosciences Commons

\section{Recommended Citation}

Mozhui, Khyobeni , "Multiscale Genomic Analysis of the Corticolimbic System: Uncovering the Molecular and Anatomic Substrates of Anxiety-Related Behavior" (2009). Theses and Dissertations (ETD). Paper 180. http://dx.doi.org/10.21007/etd.cghs.2009.0219.

This Dissertation is brought to you for free and open access by the College of Graduate Health Sciences at UTHSC Digital Commons. It has been accepted for inclusion in Theses and Dissertations (ETD) by an authorized administrator of UTHSC Digital Commons. For more information, please contact jwelch30@uthsc.edu. 


\title{
Multiscale Genomic Analysis of the Corticolimbic System: Uncovering the Molecular and Anatomic Substrates of Anxiety-Related Behavior
}

\begin{abstract}
Genetic diversity generates variation at multiple phenotypic levels, ranging from the most basic molecular to higher-order cognitive and behavioral traits. The far-reaching impact that genes have on higher traits is apparent in several neuropsychiatric conditions such as stress and anxiety disorders. Like most, if not all, neural phenotypes, stress, anxiety, and other emotion-related traits are extremely complex and are defined by the interplay of multiple genetic, environmental, experiential, and epigenetic factors.
\end{abstract}

The work presented in this dissertation is a multi-scalar, integrative analysis of the molecular and neuroanatomic substrates that underlie emotion-related behavior. The amygdala is a principle component of the limbic system that controls emotionality. Using BXD recombinant inbred (RI) mice as model organisms, the anatomy and cellular architecture of the amygdala-specifically, the basolateral amygdala (BLA) - was examined to assess the level of structural variation in this brain region. Quantitative trait locus (QTL) analysis was done to identify genetic loci that modulate the neuroanatomical traits of the BLA. The BXD RI mice were also tested using a variety of behavioral assays, and this showed a significant association between the BLA size and emotion-related behavior. The effect of chronic stress on subsequent behavior and endocrine-response was also examined in several genetically diverse inbred mice.

Finally, to explore the molecular mediators of stress and anxiety, microarrays were used to assay gene expression in three key corticolimbic brain regions-the prefrontal cortex, amygdala, and hippocampus. Several large transcriptome data sets were also analyzed. These expression data sets brought focus on an interval on mouse distal chromosome 1 that modulates diverse neural and behavioral traits, and also controls the expression of a plethora of genes. This QTL rich region on mouse distal chromosome 1 (Qrr1) provides insights into how the information in the DNA sequence is conveyed by networks of coregulated genes that may in turn modulate networks of inter-related phenotypes.

\section{Document Type}

Dissertation

Degree Name

Doctor of Philosophy (PhD)

\section{Program}

Anatomy and Neurobiology

\section{Research Advisor}

Robert W. Williams, Ph.D.

\section{Keywords}

anxiety, complex traits, expression genetics, QTL, stress

\section{Subject Categories}

Anatomy | Medical Sciences | Medicine and Health Sciences | Mental and Social Health | Nervous System | Neurosciences 


\title{
MULTISCALE GENOMIC ANALYSIS OF THE CORTICOLIMBIC SYSTEM: UNCOVERING THE MOLECULAR AND ANATOMIC SUBSTRATES OF ANXIETY-RELATED BEHAVIOR
}

\author{
A Dissertation \\ Presented for \\ The Graduate Studies Council \\ The University of Tennessee \\ Health Science Center

\begin{abstract}
In Partial Fulfillment
Of the Requirements for the Degree

Doctor of Philosophy

From The University of Tennessee
\end{abstract}

By

Khyobeni Mozhui

2009 
Chapter 2 (c) 2007 by Springer Netherlands Chapter 3 (C) 2008 by Nature Publishing Group Chapter 5 (C) 2008 by Mozhui et al. All other material (C) 2009 by Khyobeni Mozhui All rights reserved 


\section{ACKNOWLEDGEMENTS}

My heartfelt gratitude to my research supervisor and mentor, Dr. Rob Williams, for the guidance and inspiration I have received from him. I am thankful for the many opportunities he has provided to develop my scientific faculties. Beyond his role as my research adviser, he has also instilled in me a sense of confidence and independence. I consider him a rare and brilliant teacher.

I also thank all the members of my committee, Drs. John Boughter, Eldon Geisert, Kristin Hamre, and Jeff Steketee, for their advice and their critical and constructive comments. I feel most fortunate to have this group to guide me during the course of my research.

I must also thank Drs. Susan Brasser and Chris Lemon. They were my guides during the early stages of my studies. And I express my most sincere gratitude to Dr. Matt Ennis for giving me courage and counsel, and to Dr. Bill Armstrong for his support during unexpected and trying times. And to Dr. David Smith, I remain thankful.

I extend my gratitude to Dr. Lu Lu, Dr. Daniel Ciobanu, Arthur Centeno, and all the other members of the Williams' Lab. Their help have been invaluable. I am also thankful to Dr. Andrew Holmes for being such a beneficial collaborator.

I acknowledge all the funding sources that have sponsored my graduate studies (NIAAA INIA grants grants U01AA13499, U24AA13513, and U01AA014425; NIDA, NIMH, and NIAAA grant P20-DA 21131; NCI NMHCC grant U01CA105417).

Finally, to my dear ones, I am truly grateful. Apvü and Apo for their ceaseless love, their blessings, and their fervent prayers. Dear Sis, for the courage and strength I draw from her. And Ashley, for her cherished company, her encouraging words, and her excitement over my research and dissertation. 


\begin{abstract}
Genetic diversity generates variation at multiple phenotypic levels, ranging from the most basic molecular to higher-order cognitive and behavioral traits. The far-reaching impact that genes have on higher traits is apparent in several neuropsychiatric conditions such as stress and anxiety disorders. Like most, if not all, neural phenotypes, stress, anxiety, and other emotion-related traits are extremely complex and are defined by the interplay of multiple genetic, environmental, experiential, and epigenetic factors.

The work presented in this dissertation is a multi-scalar, integrative analysis of the molecular and neuroanatomic substrates that underlie emotion-related behavior. The amygdala is a principle component of the limbic system that controls emotionality. Using $\mathrm{BXD}$ recombinant inbred (RI) mice as model organisms, the anatomy and cellular architecture of the amygdala — specifically, the basolateral amygdala (BLA) — was examined to assess the level of structural variation in this brain region. Quantitative trait locus (QTL) analysis was done to identify genetic loci that modulate the neuroanatomical traits of the BLA. The BXD RI mice were also tested using a variety of behavioral assays, and this showed a significant association between the BLA size and emotionrelated behavior. The effect of chronic stress on subsequent behavior and endocrineresponse was also examined in several genetically diverse inbred mice.

Finally, to explore the molecular mediators of stress and anxiety, microarrays were used to assay gene expression in three key corticolimbic brain regions - the prefrontal cortex, amygdala, and hippocampus. Several large transcriptome data sets were also analyzed. These expression data sets brought focus on an interval on mouse distal chromosome 1 that modulates diverse neural and behavioral traits, and also controls the expression of a plethora of genes. This QTL rich region on mouse distal chromosome 1 (Qrrl) provides insights into how the information in the DNA sequence is conveyed by networks of co-regulated genes that may in turn modulate networks of inter-related phenotypes.
\end{abstract}




\section{TABLE OF CONTENTS}

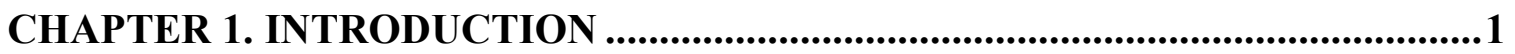

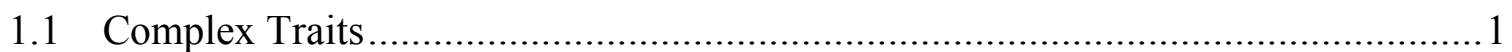

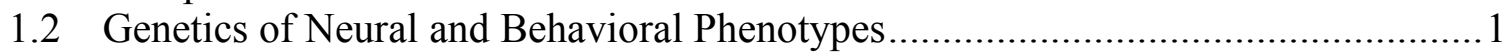

1.3 Neuroanatomy of Stress and Emotion-Related Behavior ........................................2

1.4 Mapping Loci that Modulate Complex Traits.....................................................

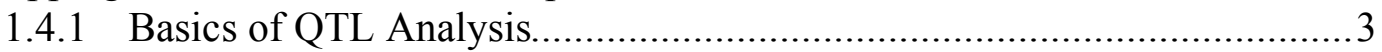

1.4.2 Inbred and Recombinant Inbred Mice..................................................

1.5 Systems Genetics Approach to Complex Traits.......................................................

1.5.1 Gene Expression as an Intermediate Phenotype .....................................6

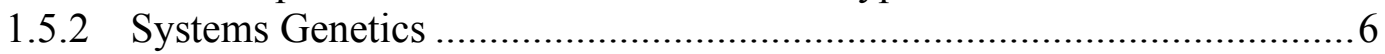

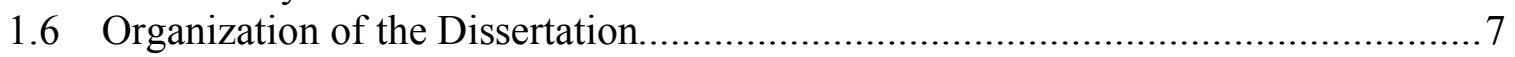

\section{CHAPTER 2. GENETIC AND STRUCTURAL ANALYSIS OF THE} BASOLATERAL AMYGDALA COMPLEX IN BXD RECOMBINANT

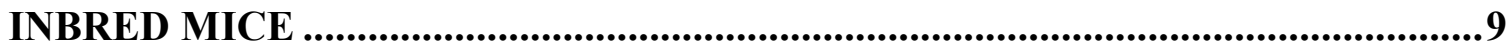

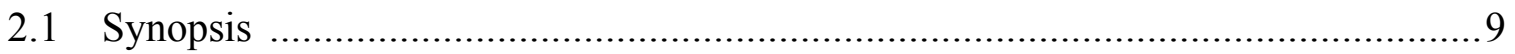

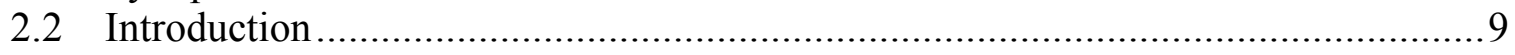

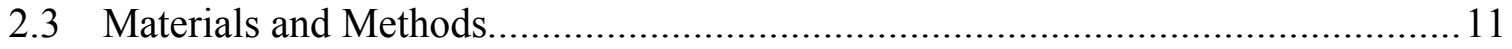

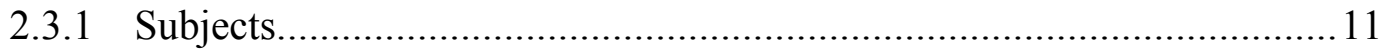

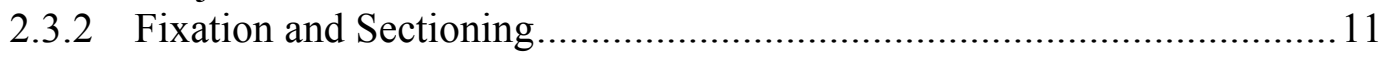

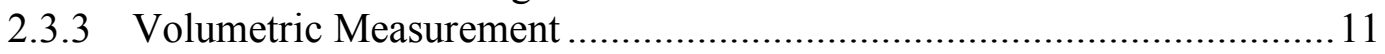

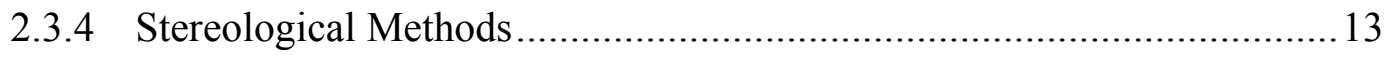

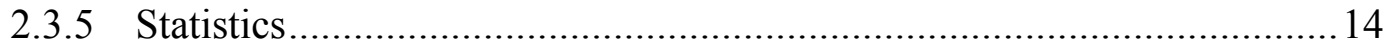

2.3.6 Genotyping and QTL Mapping......................................................... 14

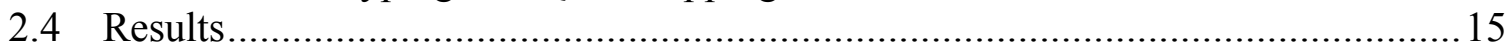

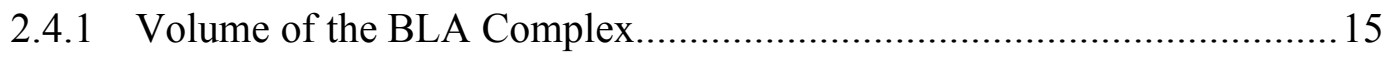

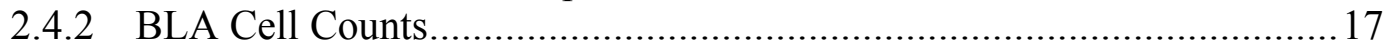

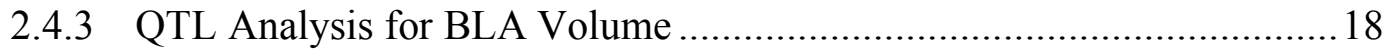

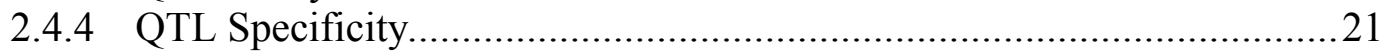

2.4.5 Correlations between BLA Volume and Cell Populations........................21

2.4.6 Neuroanatomical Correlates of BLA and Comparative QTL Analysis ....24

2.4.7 Behavioral Correlates of BLA and Comparative QTL Analysis..............26

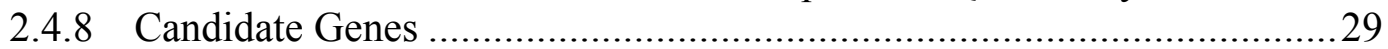

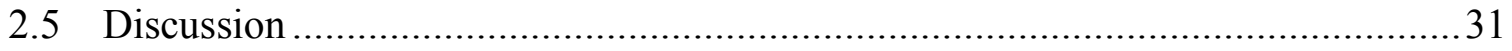

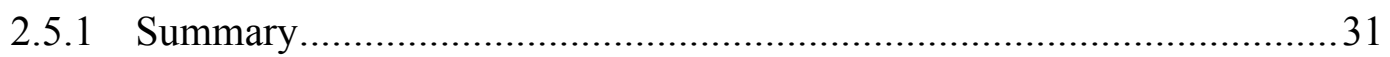

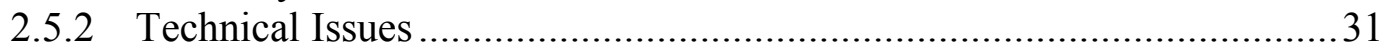

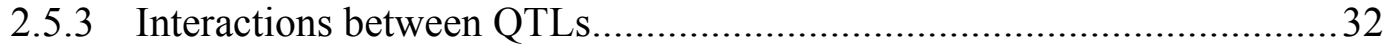

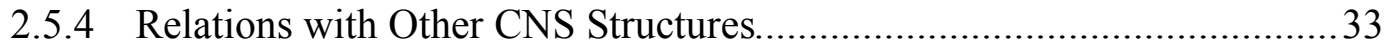

2.5.5 Implications for Behavioral Phenotypes ................................................ 33

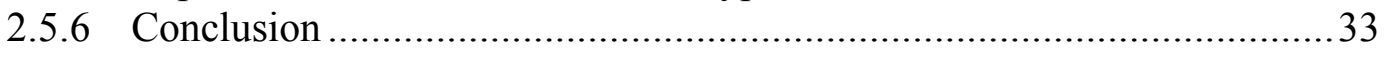




\section{CHAPTER 3. VARIATION IN MOUSE BASOLATERAL AMYGDALA VOLUME IS ASSOCIATED WITH DIFFERENCES IN STRESS REACTIVITY AND FEAR LEARNING ........................................................35}

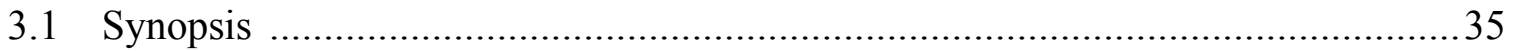

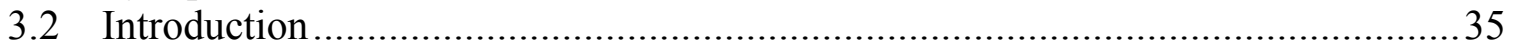

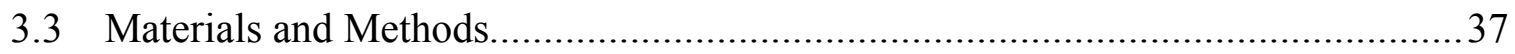

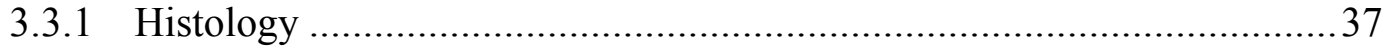

3.3.2 Selection of BXD RI Lines and Assignment to BLA Volume Groups..... 37

3.3.3 Animal Husbandry and Behavioral Phenotyping ...................................39

3.3.4 Pavlovian Fear Conditioning ......................................................... 40

3.3.5 Depression-Related Behavior and Glucocorticoid Responses to Stress ... 40

3.3.6 Anxiety-Related Behavior .............................................................. 41

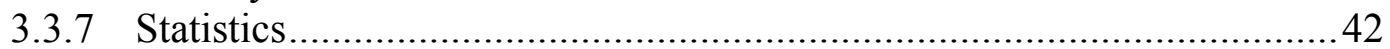

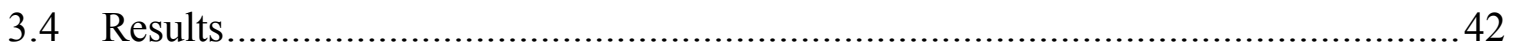

3.4.1 Pavlovian Fear Conditioning ....................................................... 42

3.4.2 Depression-Related Behavior and Glucocorticoid Responses to Stress ... 42

3.4.3 Tests for Anxiety-Related Behavior............................................... 42

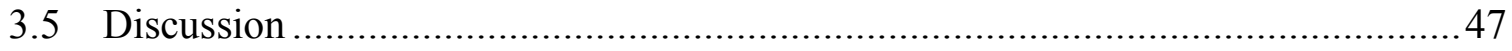

\section{CHAPTER 4. GENETIC VARIATION IN STRESS SUSCEPTIBILITY IS ASSOCIATED WITH DIVERGENT CORTICOLIMBIC GENE

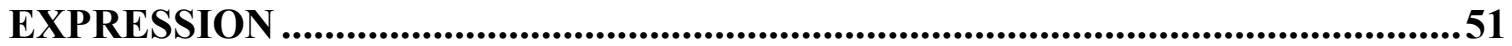

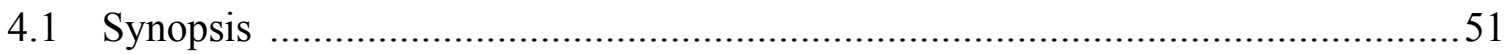

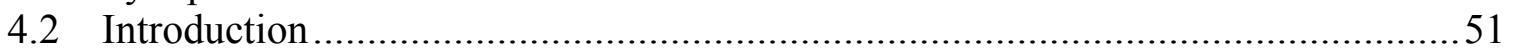

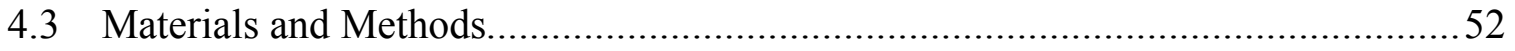

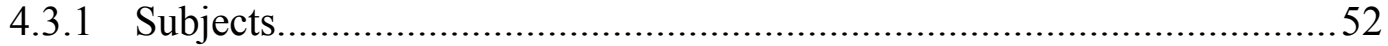

4.3.2 Repeated Restraint Stress ...............................................................53

4.3.3 Effect of Repeated Restraint on Body Weight.....................................53

4.3.4 Effect of Repeated Restraint on Anxiety-Related Behavior.....................53

4.3.5 Effect of Repeated Restraint on Depression-Related Behavior................54

4.3.6 Effect of Repeated Restraint on Swim Stress Activation of HPA-Axis ... 54

4.3.7 Sample Preparation for Expression Analysis ......................................54

4.3.8 Microarray Data Analysis..........................................................55

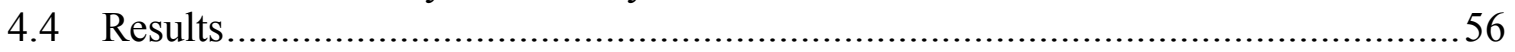

4.4.1 Effect of Repeated Restraint on Body Weight....................................56

4.4.2 Effect of Repeated Restraint on Anxiety-Related Behavior....................56

4.4.3 Effect of Repeated Restraint on Depression-Related Behavior................57

4.4.4 Effect of Repeated Restraint on the HPA-Axis ..................................57

4.4.5 Corticolimbic Gene Expression in B6 and D2....................................61

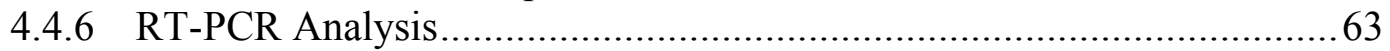

4.4.7 Cadidate Genes in BLA Volume QTL .................................................64

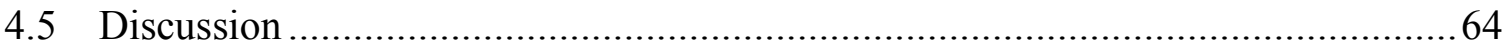




\section{CHAPTER 5. DISSECTING A QTL HOTSPOT ON MOUSE DISTAL CHROMOSOME 1 THAT MODULATES NEUROBEHAVIORAL PHENOTYPES AND GENE EXPRESSION ....................................................71}

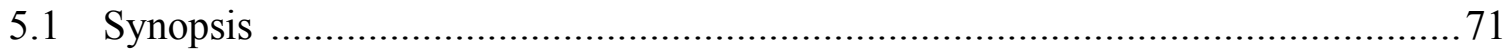

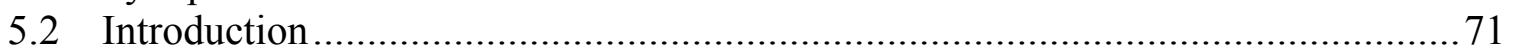

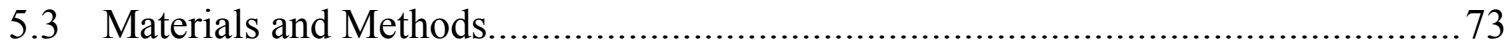

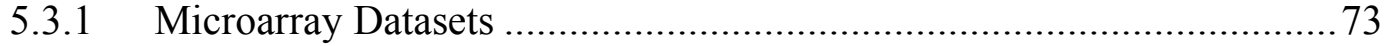

5.3.2 Mouse Strains and Genotype Data................................................... 76

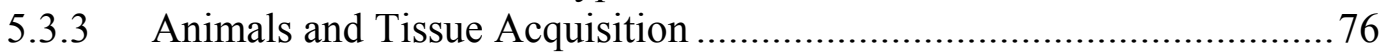

5.3.4 RNA Isolation and Sample Preparation......................................... 76

5.3.5 Microarray Probe Set Annotation ................................................... 77

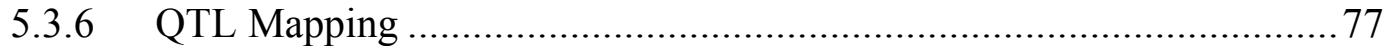

5.3.7 Screening Local QTLs...................................................................... 77

5.3.8 Analysis of Allele Specific Expression Difference.............................. 78

5.3.9 SNP Analysis in Multiple Crosses ................................................ 78

5.3.10 Partial Correlation Analysis............................................................. 78

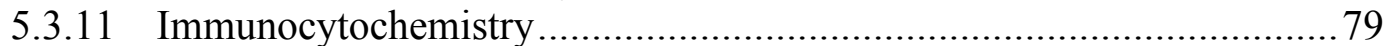

5.3.12 Microarray Analysis of Fmn2-Knockout Mice..................................... 79

5.3.13 Bioinformatics Tools ................................................................. 80

5.3.14 Control for Non-Syntenic Association and Paralogous Regions ............80

5.4 Results.

5.4.1 Enrichment in Expression QTLs in Neural Tissues .............................81

5.4.2 Replication of Trans-QTLs in Multiple Data Sets ............................. 82

5.4.3 Candidates in Qrr1....................................................................... 84

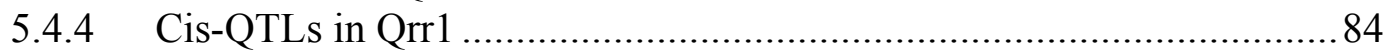

5.4.5 High-Resolution Cis-QTL Mapping ............................................ 87

5.4.6 An Aminoacyl-tRNA Synthetase Trans-QTL in Distal Qrr1 .................91

5.4.7 Trans-QTLs for Transcripts Localized in Neuronal Processes...............91

5.4 .8 tRNAs in Qrr1 .....................................................................93

5.4.9 Sequence Analysis of Multiple Crosses .........................................93

5.4.10 High-Ranking Candidates Based on Cross-Specificity of Cis-QTLs .....95

5.4.11 Partial Correlation Analysis............................................................. 95

5.4.12 Effect of Fmn2-Deletion on Gene Expression...................................96

5.4.13 Sub-Cellular Localization of FMN2 in Hippocampal Neurons..............96

5.4.14 Linking Expression and Classical QTLs: Szs1 ................................96

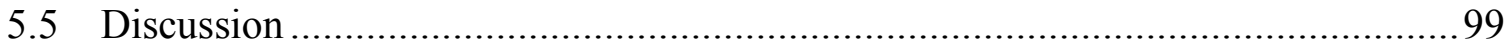

5.5.1 Kcnj9 vs. Kncj10 and Seizure Susceptibility ................................. 101

5.5.2 Multiple Loci in a Major QTL Interval............................................ 101

5.5.3 Candidates in Qrr1d and Possible Link with Local Protein Synthesis.... 102

CHAPTER 6. SUMMARY AND CONCLUDING REMARKS...........................103

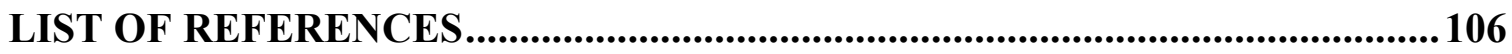

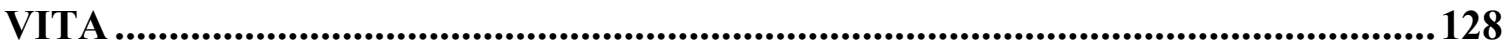




\section{LIST OF TABLES}

Table 2-1 Basolateral amygdala volume and cell number..................................... 16

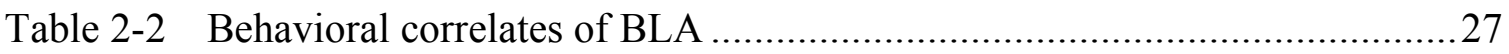

Table 3-1 Genetic correlations between BLA, striatum, hippocampus, cerebellum

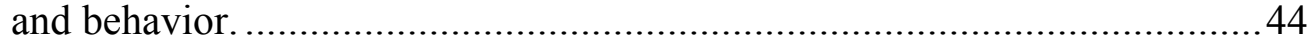

Table 4-1 Restraint-stress induced upregulation of clock genes.............................63

Table 4-2 Fold-difference scores for microarray and quantitative PCR .....................65

Table 5-1 Expression QTLs in Qrr1 in different crosses and tissues ........................74

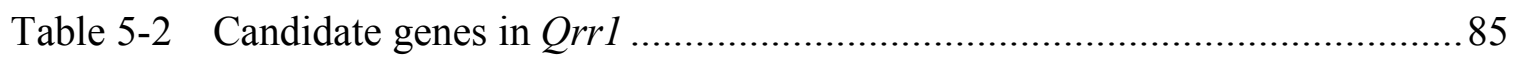

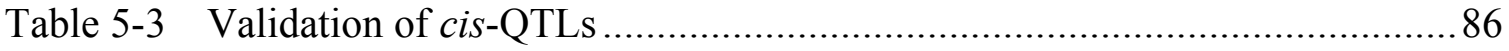

Table 5-4 Aminoacyl-tRNA synthetases that have trans-QTLs in Qrr1 ....................92

Table 5-5 Genes differentially expressed between Fmn2 $2^{+/+}$and Fmn2 $2^{-/-}$...................97

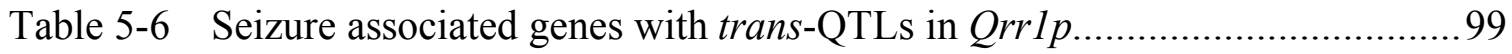




\section{LIST OF FIGURES}

Figure 1-1 Genetic segregation in F2 intercrosses and RI strains..............................

Figure 2-1 Neuroanatomy of the basolateral amygdala ............................................ 12

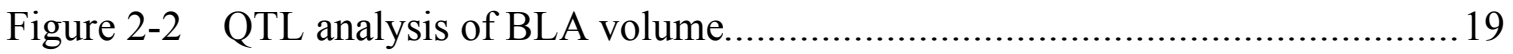

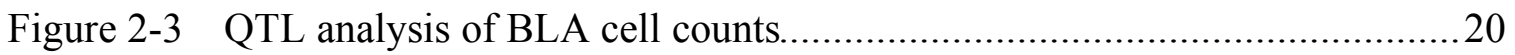

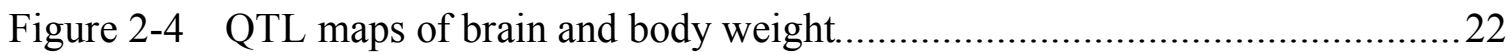

Figure 2-5 Correlations between neuroanatomical traits of the BLA ...........................23

Figure 2-6 Correlations between BLA volume and other brain structures....................25

Figure 2-7 Correlations between BLA and behavioral phenotypes ............................28

Figure 2-8 Correlations between NeuroD2 and BLA traits......................................... 30

Figure 3-1 Basolateral amygdala volume differences in BXD RI lines.........................38

Figure 3-2 Pavlovian fear conditioning in mice differing in BLA volume ....................43

Figure 3-3 Depression-related behavior and glucocorticoid responses to stress in

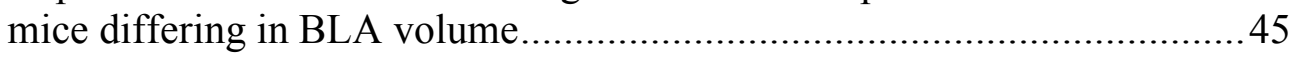

Figure 3-4 Exploratory- and anxiety-like behaviors in mice differing in BLA volume .46

Figure 4-1 Differences in repeated restraint-induced changes in body weight .............56

Figure 4-2 Differences in anxiety-like behavior in emergence test ..............................58

Figure 4-3 Differences in depression-related behavior ...........................................59

Figure 4-4 Differences in corticosterone responses to swim stress .............................60

Figure 4-5 Hierarchical cluster analysis of expression data .........................................61

Figure 4-6 Differences in corticolimbic gene expression...........................................62

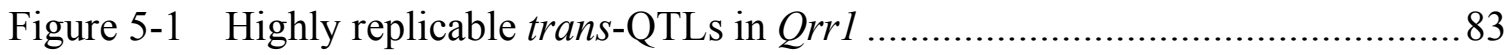

Figure 5-2 Haplotype maps of Qrrl recombinant BXD strains...................................8

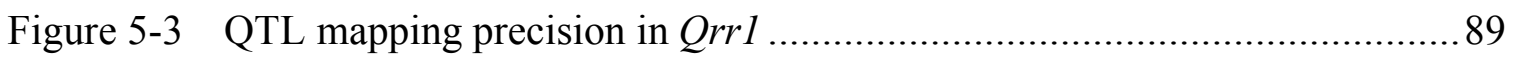

Figure 5-4 QTL for aminoacyl-tRNA synthetase in distal Qrrl ................................90

Figure 5-5 SNP comparison of multiple crosses........................................................ 94

Figure 5-6 Expression of FMN2 protein in hippocampal neurons ..............................98

Figure 5-7 Expression pattern of seizure-related genes with QTLs in Qrr1 ............... 100 


\section{CHAPTER 1. INTRODUCTION}

\subsection{Complex Traits}

A major goal of genetics is to understand how variation in DNA sequence gives rise to the myriad of individual characteristics that influence traits such as vulnerability to diseases, physiological makeup, susceptibility to substance abuse and addiction, and even personality and behavior. There are few cases in which the link between genotype and phenotype is simple enough that a trait can be traced to a single causal mutation. For example, sickle cell anemia, the first disease identified to have a genetic basis, is caused by a single point mutation in the $\beta$-globin gene (Pauling et al., 1949; Ingram, 1958). However, unlike such a monogenic trait, most traits and diseases are complex and result from the interplay of multiple genetic, epigenetic, and environmental factors. Complex diseases with polygenics origins include metabolic disorders like hypercholesterolemia and diabetes, and neuropsychiatric disorders like schizophrenia, bipolar disorder, and stress and anxiety disorders (Riley \& Kendler, 2006; Hovatta \& Barlow, 2007; Sklar et al., 2008).

Even in cases in which a single gene is strongly associated with a particular trait, the consequence on the phenotype may vary depending on the type of allelic variation. For example, a rare functionally deleterious knockout mutation in monoamine oxidase A (MAOA), a gene involved in neurotransmitter degradation, has been shown to cause violent aggressive behavior in males (Brunner et al., 1993). While this starkly demonstrates the profound influence genetic variation can have on human behavior, a more subtle effect of MAOA is seen when the DNA sequence variation is in the promoter region and effects variation in transcription efficiency rather than protein function (Caspi et al., 2002). This results in either high or low mRNA levels in individuals carrying the different alleles. The low expressing variant is associated with violent behavior but this behavioral trait develops only in males who experienced childhood mistreatment (Caspi et al., 2002). This underscores the fact that the path from the DNA to phenotype is rarely straight forward, and the penetrance of a phenotype often depends on environmental conditions.

\subsection{Genetics of Neural and Behavioral Phenotypes}

The brain is arguably the most complex organ in the human body. A large fraction of the mammalian genome, possibly over $75 \%$ of all genes, is recruited in the development and maintenance of the brain (Sutcliffe et al., 1988; Peirce \& Williams, 2006). Buttressed by this complex genetics, the brain has an intricate cytoarchitectur, composed of multiple morphologically discreet regions. It is the neurophysiology within each brain region and the circuitries between them that ultimately mediate the manifold functions of the brain and give rise to complex psychological traits and behavior. 
A fundamental question is - how do structural and physiological differences in the neural structures affect function and behavior? And what are the genetic factors that contribute to these variations? Psychological traits such as emotional temperament and behavioral phenotypes show significant inter-individual variations. In addition to environmental factors and life experiences, multiple genetic factors play important roles in defining the emotionality and behavior of an individual (Gershenfeld \& Paul, 1998). The heritability of personality traits has been estimated to be as high as $30-60 \%$ (Bouchard, 1994; Gershenfeld \& Paul, 1998). It is the genetic differences that make one individual more vulnerable than another to stressful experiences, and confer risk factors for different neuropsychiatric conditions such as depression, anxiety disorders, and addiction (Charney \& Manji, 2004; Goldman et al., 2005; Hariri \& Holmes, 2006; Leygraf et al., 2006).

The mouse has been extensively used as a model organism to study the genetics of behavior (Gershenfeld \& Paul, 1998; Willis-Owen \& Flint, 2006). Studying behavior in mice affords several advantages that are lacking in human studies. These include the ability to strictly control the environment of the animal, to carry out directed mating, and the availability of several inbred strains with diverse phenotypic profiles. Different behavioral measures have been used as indicators of cognition and emotionality in rodents, e.g. open field activity, elevated plus maze, light/dark environment preference, fear conditioning, forced swim test etc. These studies have uncovered complex genetics underlying behavior with multiple loci modulating stress responsiveness, fear, and anxiety levels in mice (Willis-Owen \& Flint, 2006). Significant concordance between human and mouse traits also exists, e.g., the gene Rgs 2 was shown to modulate anxiety in mice, and was subsequently found to be associated with anxiety in human (Yalcin et al., 2004; Smoller et al., 2008).

\subsection{Neuroanatomy of Stress and Emotion-Related Behavior}

The amygdala is a neural structure central to the experience of emotions and expression of emotional behavior. Sensory inputs with emotional components are transmitted to the amygdala where they are processed and further relayed to other regions to modulate autonomic and behavioral responses, and to form emotional memories (LeDoux, 2000; Rosen, 2004). As a neural substrate of emotionality, many neuropsychiatric disorders have been associated with structural changes in the amygdala. Individuals with genetically predisposed susceptibility to anxiety and depression have been shown to have higher amygdala reactivity and smaller amygdala volumes (Pezawas et al., 2005). Structural changes in the amygdala have also been associated with traumatic stress disorder, bipolar disorder, and aggressive behavior (Hayek et al., 2005; Pezawas et al., 2005; Meyer-Lindenberg et al., 2006). In addition to the amygdala, abnormalities in structures such as the prefrontal cortex, hippocampus and other components of the limbic system have also been associated with several neuropsychiatric disorders (Drevets, 2000; Hajek et al., 2005; Vermetten et al., 2006). 
Such associations between neuroanatomy and disease state however do not clarify whether the structural abnormalities are the cause or effect of neuropathological conditions. Some studies are beginning to indicate that the neuroanatomical traits may indeed be predisposing factors for certain disorders. For example, twin studies show that smaller hippocampal volume, rather than being a consequence of stress, may be a genetically determined phenotype that predisposes an individual to a higher risk for developing stress-related disorders (Gilbertson et al., 2002). Structural abnormalities in the amygdala and prefrontal cortex may also be preexisting risk factors for bipolar disorder (Hajek et al., 2005). These observations imply that some genetic factors may have pleiotropic influence on both neuroanatomy and behavior. The genetic analysis of relevant neural structures may therefore be invaluable in identifying the genes that modulate behavior.

Studies in mice also show that neuroanatomical phenotypes such as the volume and cellular composition of the amygdala are highly variable traits with complex underlying genetics (Mozhui et al., 2007). And such natural variations in the anatomical phenotypes of the amygdala serve as significant predictors of stress-reactivity and fearrelated behaviors (Yang et al., 2008). These studies highlight the inextricable link between genetic factors and phenotypes at the structural, physiological, and behavioral levels, and calls for an integrative approach to analyze complex traits.

\subsection{Mapping Loci that Modulate Complex Traits}

\subsubsection{Basics of QTL Analysis}

The large majority of biological traits are polygenic and show quantitative (or continuous), rather than qualitative (or discreet) variations. For example, body weight, brain size, levels of anxiety etc., are traits that show quantitative differences among individuals. The genetic regions that harbor the gene variants that contribute to such quantitative variations are referred to as quantitative trait loci or QTLs (Lynch \& Walsh, 1998). Both QTLs and Mendelian mutations are, simply put, genetic polymorphisms that lead to phenotypic variations; the main distinction between the two types of loci lies in the effect size, i.e. the amount of phenotypic variance generated by each locus. In contrast to Mendelian phenotypes, complex traits are shaped by the action of multiple QTLs, each QTL contributing to only a portion of the variance. In case of behavioral traits, a single QTL is estimated to usually contribute to less than $10 \%$ of behavioral variance (Flint, 2003).

Identifying the genetic loci that modulate a trait based on correlation between variation in phenotype and variation in genotype is the essence of genetic mapping. This first involves systematically genotyping a genetically diverse population using microsatellite or SNP markers. The phenotype of interest is then measured and its variability in the population assessed. A statistical test is then carried out to identify chromosomal regions that segregate with the trait and show linkage with the trait, i.e., 
identify genetic regions that have the same genotype among individuals with similar trait values but differ between individuals with dissimilar trait values. The statistical significant of linkage of a QTL with the trait is expressed as the logarithm of odds ratio (LOD) or likelihood ratio statistic (LRS) scores with associate p-value.

\subsubsection{Inbred and Recombinant Inbred Mice}

An invaluable resource in the genetic dissection of complex traits is the availability of inbred strains of mice that can be used to generate experimental populations. At the initial step, two inbred strains with divergent phenotypes are selected as parental strains. To illustrate, $\mathrm{C} 57 \mathrm{BL} / 6 \mathrm{~J}(\mathrm{~B} 6)$ is a relatively seizure resistant strain while DBA/2J (D2) is seizure susceptible (Seyfried et al., 1980). Both B6 and D2 have been densely genotyped. In order to carry out a complex trait analysis of seizure susceptibility, the two strains can be used as parental strains to derive populations in with the phenotype and causal gene variants segregate. Firstly, B6 and D2 are crossed to generate heterozygous F1 hybrids. These hybrids are generally expected to show traits intermediate between the two parents. A population of F2 intercrosses can then be derived from the F1s (Figure 1-1). The F2s represent a genetically segregating population and will show varying levels of seizure susceptibility depending on the combination of alleles inherited from each parent. QTL analysis can be carried out using such a collection of F2.

By inbreeding the F2 mice for over 20 generation, inbred mice that are recombinants of the parental genotype can be generated (Figure 1-1). An extensively used set of recombinant inbred (RI) strains is the BXD RI mice that were derived by inbreeding F2 progenies of B6 and D2 parents (Taylor, 1989; Taylor et al., 1999; Williams et al., 2001b). About a 100 different BXD RI strains, including advanced RI strains (inbred strains derived from progenitor BXD strains) have now been created for this mapping panel (Peirce et al., 2004). Such RI mice provide several advantages. Unlike F2 intercrosses, which are unique and studies with them irreproducible, RI strains are immortal colonies. The first obvious advantage is that multiple cases of the same RI strain can be phenotyped and the values averaged to control for environmental noise and technical error. Studies using RI strains can also be carried out many times under different environmental conditions or using different experimental protocols. Several different phenotypes have been measured from the BXDs over the years (http://www.genenetwork.org) and this allows comparison and correlation across data sets. For example, amygdala volume data that was collected in 2006 can be correlated with behavioral data collected in the 1990s to show significant association between the size of the amygdala and open field behavior (Jones et al., 1999; Mozhui et al., 2008). 

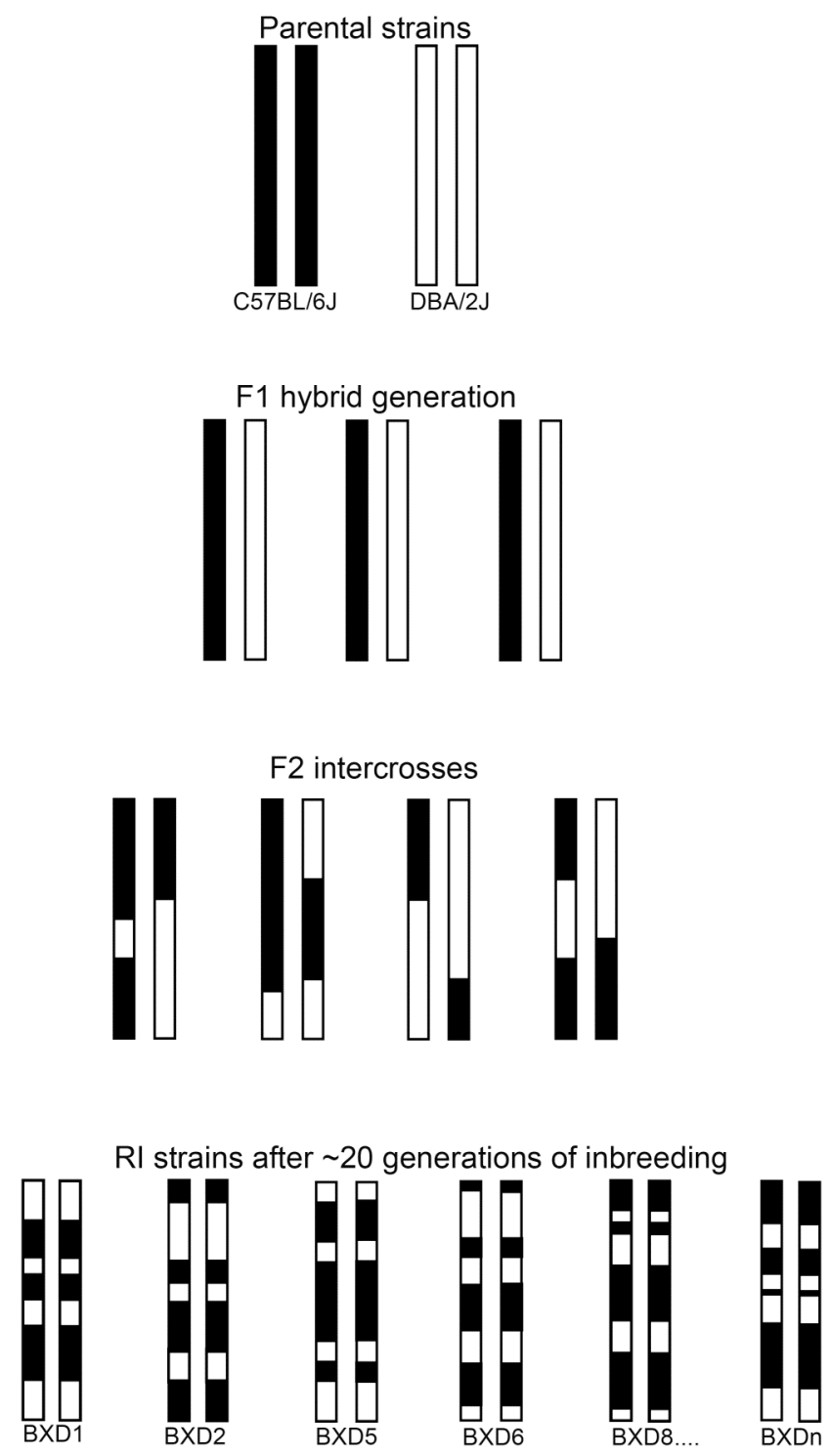

Figure 1-1 Genetic segregation in F2 intercrosses and RI strains

All pairs of autosomal chromosomes (chromosomes 1 to 19) in inbred mice are homozygous and carry identical maternal and paternal alleles. This figure illustrates one such pair of autosomal chromosome. F1 hybrid crosses are made by mating two parental inbred strains, in this case C57BL/6J (B6) and DBA/2J (D2). All mice in the F1 generation are isogenic and inherit one allele from B6 and the other from D2. Because of reciprocal crossover events, the $\mathrm{F} 1$ gametes contain chromosomes that are recombinants of the two parental chromosomes and mating the F1 mice generates a genetically segregating F2 generation. The F2 mice can then be inbred to homozygosity over several generations to form strains of inbred mice that are recombinants of the parental chromosomes, and hence termed recombinant inbred (RI) strains. 


\subsection{Systems Genetics Approach to Complex Traits}

\subsubsection{Gene Expression as an Intermediate Phenotype}

Genetic variation can cause marked differences in gene expression among individuals. By setting the rate of transcription and mRNA degradation, and coding for allelic variants and alternative splicing, the DNA sequence essentially directs the amount and type of RNA and protein produced by each gene. The quantitative and qualitative differences in mRNAs and proteins can be considered as phenotypes that are at the most basic molecular level (Damerval et al., 1994; de Vienne et al., 1994). Since it is from this milieu of molecules that all higher phenotypes emerge, understanding how genetic variation modulates gene expression is fundamental to understanding the molecular origins of complex traits.

Microarray technology has been a boon in the study of gene expression. This technology has made it possible to efficiently profile the expression level of numerous genes in genetically diverse populations of organisms, including human and mice. Such transcriptomic data have been generate for a wide variety of cell types and tissues, including several different neural tissues from experimental mouse populations (Hitzemann et al., 2004; Chesler et al., 2005; Peirce et al., 2006; Yang et al., 2006). The expression level of an mRNA species can be highly variable and heritable in nature (Brem et al., 2002; Schadt et al., 2003; Chesler et al., 2005). Similar to organism-level phenotypes (now termed as 'classical phenotypes' to distinguish them from molecular phenotypes), expression traits can exhibit Mendelian or multigenic inheritance patterns and are therefore amenable to QTL analysis.

One aspect of expression traits that differs from classical phenotypes is that each mRNA and protein species has a corresponding genetic locus that harbors the parent gene. Because of this, a QTL that modulates gene expression can either be located close to the parent gene or at a distance from the parent gene. A QTL that maps close to the parent gene indicates $c i s$-acting regulatory mechanisms ( $c i s-\mathrm{QTL}$ ), and implicates sequence variations within or close to the gene (e.g. in regulatory elements such as transcription factor binding sites) as being responsible for the variation in transcript level. A QTL that maps at a distance from the parent gene indicates trans-acting regulatory mechanisms controlling the expression of the gene (trans-QTLs).

\subsubsection{Systems Genetics}

The information in the DNA sequence is transmitted via networks of genes and molecular pathways and can have influence at multiple phenotypic levels. With the generation of transcriptome data sets, it is now possible to analyze gene expression on a global scale and identify gene networks that may be the molecular correlates of organism level phenotypes (Bystrykh et al., 2005; Chesler et al., 2005; Schadt et al., 2005). This has led to the conception of systems genetics, an integrative analytical approach that 
attempts to develop a more comprehensive view of an organism by collectively studying many phenotypes and multiple genetic and environmental factors (Williams, 2006). Systems genetics has been defined as "the integrative study of a multitude of gene variants and a rich admixture of phenotypes." Each phenotype is considered within the backdrop of many other interrelated phenotypes, and associated gene networks.

Such an integrative approach to complex traits has become feasible only with the production of large genomics resources such as dense genotype and SNP sequences, and transcriptome and proteome data sets. Another area that has been vital to systems genetics has been the development of bioinformatics resources that curate large data sets, and provide sophisticated computational tools and analytical systems (e.g. the genome browser at http://genome.ucsc.edu, Mouse Phenome at http://www.phenome.jax.org, GeneNetwork at http://www.genenetwork.org).

\subsection{Organization of the Dissertation}

The work presented in this dissertation is a systems genetics approach to study neuroanatomical traits in relation to emotion-related behavior. The main focus is on the basolateral amygdala (BLA), but several other brain regions, including the prefrontal cortex, hippocampus, and striatum, have also been considered.

To begin with, Chapter 2 examines the morphology and cytoarchitecture of the BLA in a panel of 35 BXD RI and parental strains, and F1 hybrids (Mozhui et al., 2006). This analysis revealed significant variation in structural traits of the BLA, and identified a significant QTL on chromosome associated with the volume of the BLA. In addition to the QTL on chromosome 8, few other suggestive QTLs were also detected.

In Chapter 3, the natural variations in the BLA volume are associated with stress reactivity and fear learning behavior (Yang et al., 2008). This study showed that mice with smaller BLA exhibited more pronounced fear learning and released more corticosterone in response to stress. This demonstrates that structural trait of a brain region can have a significant influence on behavior.

Chapter 4 examines the effect of chronic stress on subsequent behavior and endocrine response. To introduce more genetic diversity, seven inbred strains, including B6 and D2, were tested using behavioral assays of anxiety- and depression-related behavior. Gene expression in the BLA, PFC, and hippocampus of B6 and D2 mice was also evaluated. The expression study detected different sets of stress-sensitive genes in B6 and D2. This study especially highlighted several genes involved in controlling the circadian rhythm (e.g. Per1, Per2, Per3). Many of the stress sensitive genes are also known to be associated with neurological disorders in human.

In Chapter 5, the focus is shifted to a genetic region that harbors many QTLs. This chapter examines the QTL rich on mouse distal chromosome 1 or Qrrl (Mozhui et al., 2008). This QTL region appears to harbor multiple loci that control both gene 
expression and several neural traits, including the volume of the hippocampus, and several emotion-related behavioral traits. Many genes detected to be differentially expressed between B6 and D2 are also located in this region. By apply high-resolution mapping and by examining multiple crosses, Qrrl was subdivided into two broad QTL regions. Formin $2(F m n 2)$ was identified as a high priority candidate gene that may be the chief modulator of expression traits that map to Qrrl.

Finally, Chapter 6 summarises the results and gives a concluding discussion of the work reported in this dissertation. 


\section{CHAPTER 2. GENETIC AND STRUCTURAL ANALYSIS OF THE BASOLATERAL AMYGDALA COMPLEX IN BXD RECOMBINANT INBRED MICE*}

\subsection{Synopsis}

The amygdala integrates and coordinates emotional and autonomic responses. The genetics that underlie variation in amygdala structure may be coupled to variation in levels of aggression, fear, anxiety, and affiliated behaviors. We systematically quantified the volume and cell populations of the basolateral amygdala complex (BLA) across 35 BXD recombinant inbred (RI) lines, the parental strains - C57BL/6J (B6) and DBA/2J (D2) — and F1 hybrids (n cases $=199$, bilateral analysis). Neuron number and volume vary 1.7- to 2-fold among strains (e.g., neuron number ranged from 88,000 to170,000). Glial and endothelial populations ranged more widely (5- to 8-fold), in part because of higher technical error. A quantitative trait locus (QTL) for the BLA size is located on chromosome (Chr) 8 near the Large gene. This locus may also influence volume of other regions including hippocampus and cerebellum. Cell populations in the BLA appear to be modulated more weakly by loci on Chrs 11 and 13. Candidate genes were selected on the basis of correlation with BLA traits, chromosomal location, SNP density, and expression patterns in the Allen Brain Atlas. Neurod2, a gene shown to be significant for the formation of the BLA by knockout studies, is among the candidate genes. Other candidates include Large, Neurocan, and Thra. Responses to drugs of abuse and locomotor activity were the most notable behavioral correlates of the BLA traits.

\subsection{Introduction}

The amygdala is a composite of functionally distinct nuclear groups in the forebrain. It is neuroanatomically intricate and comprised of as many as 13 different nuclei connected by complex intrinsic, and afferent and efferent connections (Pitkänen et al., 1997). It is a major constituent of the limbic system and is regarded as a crucial neural component underlying the experience of emotions and emotion-related cognitive functions (McIntyre et al., 2003; McGaugh, 2004). Evidence supports its role in the mediation of aversive and rewarding emotions (Rogan \& LeDoux, 1996; LeDoux, 2000; Calder et al., 2001; Baxter \& Murray, 2002; Lee et al., 2004), and the extensive connections it shares with the dopaminergic reward systems (Friedman et al., 2002) indicates an amygdalar contribution in the neurophysiology of reward, anxiety, motivation, and addiction (Phillips et al., 2003; Ciano \& Everitt, 2004).

A number of human psychopathological disorders are accompanied by alterations in the size and cytoarchitecture of the amygdala. Examples include abnormal reductions

\footnotetext{
* Adapted with permission from Springer. Mozhui K, Hamre KM, Holmes A, Lu L, Williams RW (2007). Genetic and structural analysis of the basolateral amygdala complex in BXD recombinant inbred mice. Behav Genet 37:223-243.
} 
in volume among patients with schizophrenia (Niu et al., 2004), bipolar disorder (Blumberg et al., 2003), depression (Rosso et al., 2005), and Williams Syndrome (Reiss et al., 2004); and abnormal increases in volume and cell density among autistic patients (Abell et al., 1999). Interestingly, recent studies suggest that functional abnormalities in the amygdala of certain patient groups may be partly of genetic origin. For example, polymorphic variants in the human serotonin transporter and monoamine oxidase A genes are associated with trait differences in amygdala volume and/or functional responses to aversive stimuli (Hariri \& Holmes, 2006; Meyer-Lindenberg et al., 2006). Thus genetic analysis of the structural variations of the amygdala may provide insights into the functional and behavioral significance of such variances.

Genetic heterogeneity generates significant variations in phenotypes ranging from expression levels of mRNAs and proteins, the size and shape of neurons and neural structures, through to innate and learned behaviors. We are just beginning to understand relations among sequence differences, environmental factors, and diverse multiscale phenotypes. Mice provide an excellent population model to study such genetic and phenotypic variability. The environment of diverse sets of strains (a test population) can be fixed or systematically varied. In particular, the set of BXD strains (Peirce et al., 2004) has proved to be an extremely valuable reference population to study networks of phenotypes and their modulation by gene variants (Chesler et al., 2003; Chesler et al., 2005). The parental strains, B6 and D2, have been sequenced, and approximately 2 million single nucleotide polymorphisms (SNPs) between them have been identified.

Complex trait studies with the BXDs are also bolstered by the availability of a broad web-based data compilation of these strains' genotypes, numerous behavioral and physiological phenotypes, and microarray expression profiles, and integrated analytical tools maintained in GeneNetwork (GN, http://www.genenetwork.org). Previous studies have used the BXD RI panel to analyze the genetic determinants of differences in size and cell number of brain structures including the cerebellum, hippocampus, neocortex, striatum, and olfactory bulb (Neumann et al., 1993; Rosen \& Williams, 2001; Airey et al., 2002; Seecharan et al., 2003; Airey et al., 2005; Li et al., 2005). In the present study, we have undertaken a complex trait analysis of the BLA. Its volume and densities of three cell types - neurons, glia, and endothelial cells - were measured from 35 BXD strains and their parental strains and F1 hybrids. In addition to identifying QTLs that may be significant for the development and structure of the BLA, we performed correlative analysis with mRNA expression levels in the brain, and with behavioral phenotypes catalogued in GN. Such an integrated study can potentially determine modulatory roles of multiple genetic factors in establishing brain morphology, and their influence on neurophysiology, function, and behavior (Chesler et al., 2003). 


\subsection{Materials and Methods}

\subsubsection{Subjects}

Measurements were taken from brain sections of a set of 35 BXD RI strains, the parental B6 and D2 strains, and F1 hybrids. The serial sections used in this study are all part of the Mouse Brain Library (MBL) collection of physical sections. Images of these sections can also be downloaded from http://www.mbl.org (Lu et al., 2001; Rosen et al., 2003; Seecharan et al., 2003). The BXD strains are completely inbred and have been generated by the repeated mating between siblings starting from the F2 stage (Taylor, 1989; Taylor et al., 1999). The animals were purchased from the Jackson Laboratory (Bar Harbor, ME) and housed in a pathogen free colony at the University of Tennessee Health Science Center. Animals were acclimatized for at least two weeks in the University of Tennessee animal facility before use. Number of animals housed in a single cage did not exceed 5 and the average cage density was 3 per cage. They were maintained on a 14/10h light-dark cycle at 20-24 $\mathrm{C}$ and fed 5\% fat Agway Prolab 3000 rat and mouse chow. The average age of mice from which data were collected was 96 days (ranging from 30 to 500 days), and a total of 96 females and 103 males were used. All procedures followed the guidelines of institutional animal care and use committee.

\subsubsection{Fixation and Sectioning}

As described previously (Airey et al., 2001; Lu et al., 2001; Seecharan et al., 2003), mice were deeply anaesthetized with Avertin, and perfused through the heart with $0.1 \mathrm{M}$ phosphate buffered saline. This was followed by solutions of: $1.25 \%$ glutaraldehyde and $1.0 \%$ paraformaldehyde in phosphate buffer $(0.1 \mathrm{M})$; and $2.5 \%$ glutaraldehyde and $2.0 \%$ paraformaldehyde in phosphate buffer. Brains were dissected out and weighed. Brain tissues were subsequently embedded in celloidin and sectioned along the coronal or horizontal planes at $30 \mu \mathrm{m}$ thickness with a sliding microtome, and stained with cresyl violet. Every tenth section was mounted on a slide and coverslipped. An interval thickness of $300 \mu \mathrm{m}$ separates adjacent sections on each slide (Rosen \& Williams, 2001). In the MBL, the majority of the cases have two sets of serial section slides per brain (slide A and slide B).

\subsubsection{Volumetric Measurement}

Volumetric measurements were taken from a total of 199 brains. An average of 5 subjects was used per strain, and with the exception of BXD37, all other strains' means are representatives of at least four subjects. For BXD37, measurements were taken from only 2 individuals due to limited availability. We decided to include the BXD37 data because its inclusion or exclusion did not have a major affect on the subsequent linkage analysis. Of the 199 cases, 175 were coronal sections and 24 were horizontal sections. 
The limited use of horizontal sections is because the outline of the BLA is more clearly definable in the coronal sections.

The amygdala is a complex neural structure and has been divided into distinct nuclear complexes, and further sub-divided into nuclei and sub-nuclei regions (Pitkänen, 2000). For this study, we took measurements from the portion of the BLA complex that is bordered laterally by the amygdalar capsule (amc) (Swanson \& Petrovich, 1998), and medially by the external capsule (ec). These clear demarcations and definability would ensure consistent morphometric measurements. This portion of the BLA complex includes the lateral nucleus, which is further subdivided into the dorsolateral (LaDL), ventrolateral ( $\mathrm{LaVL}$ ) and ventromedial (LaVM) sub-nuclei, and the basolateral nucleus, which is further subdivided into the anterior magnocellular (BLA) and posterior parvicellular (BLP) sub-nuclei (Figure 2-1A). These divisions have been made according to a standard atlas of the brain of B6 (Franklin \& Paxinos, 2001). The basomedial nucleus is also a part of the BLA complex but it was not included in our measurements because its border is less clearly definable and may have added to data inaccuracy.

NIH Image (version 1.63) was used for volumetric analysis. Images from serial sections were captured from a Zeiss light microscope to a computer. After calibrating the instrument to a standard length of $1 \mathrm{~mm}$, the border of the amygdala delineating the BLA was manually traced and area determined. Such manual measurements were taken along the entire rostrocaudal thickness of the BLA (approximately 6-9 sections for each brain) at an interval thickness of $300 \mu \mathrm{m}$. Measurements were taken bilaterally and the final values represent the left-right average. Because the majority of the cases have two sets of serial sections, the average BLA complex volume for most individual cases was

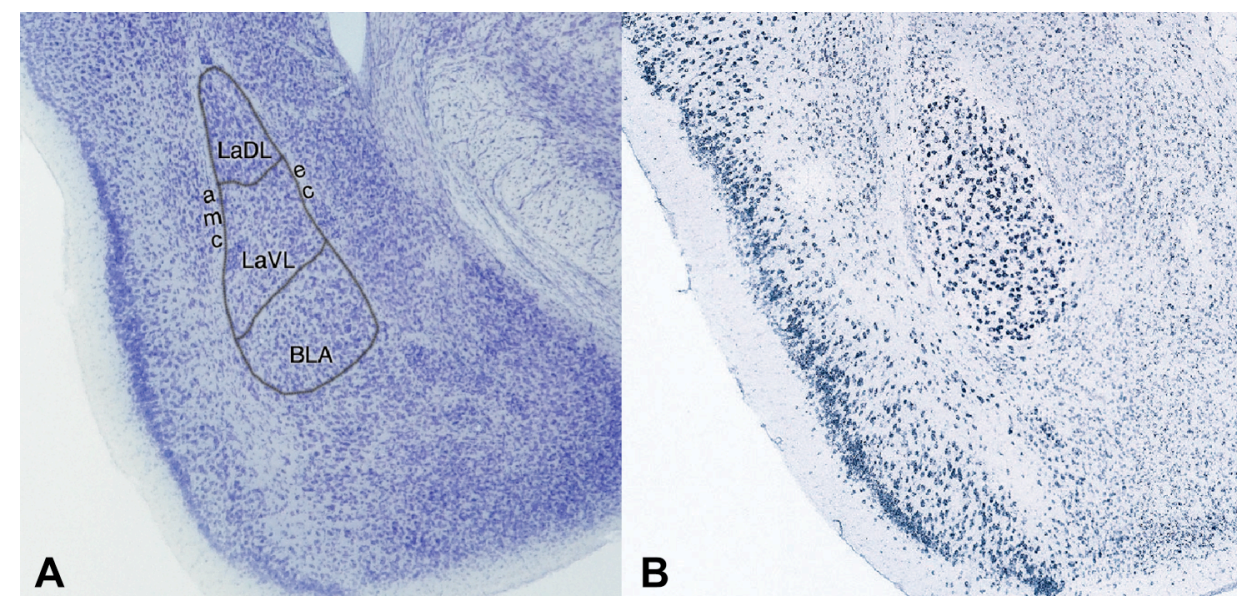

Figure 2-1 Neuroanatomy of the basolateral amygdala

(A) Nissl stained coronal section from MBL (bregma:-1.8). Outlined is the portion of the BLA complex that included the lateral nucleus (LaDL, LaVL) and the basolateral nucleus (BLA); amc: amygdalar capsule; ec: external capsule. (B) Expression of the Large gene in the BLA complex (in situ taken from Allen Brain Atlas; Large_277_030609127-2, positon: 6925). 
calculated from a total of four sets of measurements, i.e. bilateral measurement from slides $\mathrm{A}$ and $\mathrm{B}$.

To correct for the volumetric shrinkage caused by histological processing, the measured values were divided by the total brain volume and multiplied by the brain volume expected for that brain weight. The brain density of the MBL tissues is taken to be $1.05 \mathrm{mg}$ per $\mathrm{mm} 3$ of fixed tissue. The post-processing total brain volume was determined by point counting and the method is described in Williams (2000). After each individual volume measurement was corrected for shrinkage, the strain averages were determined.

\subsubsection{Stereological Methods}

We applied the three-dimensional direct cell counting technique that was developed by Williams and Rakic (1988) to estimate cell density and total number. We used the whole-section method as it gives a relatively unbiased estimate of cell number from a tissue section and avoids the error caused by differential shrinkage along the $\mathrm{z}$ axis (von Bartheld, 2001). A count box of $32 \times 33 \times 30 \mu \mathrm{m}$ was superimposed over the live image of the section, and we followed the standard counting rules defined by Gundersen (1977), and Williams and Rakic (1988). The same counting protocol was used as described previously by Seecharan et al. (2003). Distinction between cell populations was based on established criteria (Ling et al., 1973; Satorre et al., 1986) and as used by Seecharan et al. (2003). Cells were classified into three groups: neurons, glial cells, and endothelial/other cells. No distinction was made between projection neurons and interneuron. Cells whose identity could not be discerned with certainty were included in the endothelial/other group.

Stereological measurements were limited only to coronal sections from which the volumetric measurements were taken and the horizontal sections were excluded. From each serial section slide, the section that had the most complete representation of the BLA complex was chosen for cell counting. A total of 10 sites were sampled from each side of the BLA complex (2 sites for each sub-nuclei) by focusing the x100 oil objective (NA 1.25) over each sub-nuclei (LaDL, LaVL, LaVM, BLP, BLA). Counts were taken bilaterally and for each case, the final cell number represents the left and right average. The number of subjects used for each strain is lower for the stereological data at an average of 4 subjects per strain. The data for BXD37 and BXD11 represent the average of only two individuals.

To assess the validity of stereological method we compared counts obtained from right and left sides. While there will be some genuine left-right asymmetry, most variation is likely to be due to sampling and technical errors (Williams et al., 1996). Paired t-test showed no significant difference between the cell counts taken from the left and right BLA $\left(\mathrm{t}_{165}=1.72, p=0.08\right.$ for glia; $\mathrm{t}_{165}=1.15, p=0.25$ for endothelial cells; $\mathrm{t}_{166}=1.54, p=0.13$ for neurons). However, BLA volume - which we consider to be the 
most reliable data with a low coefficient of error-has a modest but statistically significant left-right asymmetry $\left(\mathrm{t}_{195}=2.5, p=0.01\right)$.

\subsubsection{Statistics}

Data were organized using a spreadsheet program (Excel) and most exploratory analysis and statistical tests were performed using Data Desk (http://www.datadesk.com). To examine effects of cofactors such as brain weight, body weight, age, and sex on the BLA, we applied multiple linear regression. In the case of the volume data, the plane of section was also included as a factor. In addition, the effect of each variable as a single factor was also examined by simple regression analysis, and the percentage of variance explained by a covariate was computed from the adjusted correlation coefficient. Individual trait values rather than strain means were used for regression analysis. Residual values were calculated only for those factors that were significant predictors. The corrected data, adjusted for these effects (residual + mean), provide a more accurate estimate of variability specific to the BLA. The adjusted data were also used for linkage analysis.

Effect of strain on trait variance was used as an estimate of trait heritability. The intra-class correlation coefficient was computed by performing an analysis of variance (ANOVA) with strain as a single factor and this provides an estimate of strain effect on trait variance.

\subsubsection{Genotyping and QTL Mapping}

The B6 and D2 strains differ significantly in sequence and approximately 2 million informative SNPs have been defined across the genome (see http://www.genenetwork.org/beta/snpBrowser.py). A subset of approximately 14,000 of these sequence variants (SNPs and microsatellites) have been used to genotype the BXD strains (Williams et al., 2001b; Peirce et al., 2004; Shifman et al., 2006; http://www.well.ox.ac.uk/mouse/INBREDS/). The BLA trait data were entered into GN and QTL mapping was performed using the WebQTL mapping module.

For linkage analysis, WebQTL performs intervals mapping using 3,795 SNP and microsatellites as markers. The non-parametric $p$-value (alpha of 0.05 ) of the linkage ratio statistic (LRS) is computed by performing 1000 or more permutations. This threshold is denoted by the upper horizontal line in linkage maps. A suggestive LRS threshold is set at a genome-wide alpha of 0.63 and is denoted by the lower horizontal line in the linkage map. Two-thousand or more bootstraps are also performed by GN to estimate the confidence limit of the location of the QTL peak interval, and the results are represented as a "frequency of peak LRS value" histogram. Another parameter computed by WebQTL is the additive effect, which is an estimate of the quantitative effect of an allele on the measured phenotype. In the case of the BXDs, a locus can have a B6 allele $(B)$ or a D2 allele $(D)$ and the additive effect is half of the difference between the means 
of homozygous cases and is calculated as: (mean of $B B$ cases)-(mean of $D D$ cases)/2. The red plot in the linkage graphs denotes a positive effect by the $B$ allele and for computational purpose, GN gives it a nominal negative value; a positive effect by $D$ allele is denoted by the green plot and is assigned a positive value. These values are in the same units as the data, in this case $\mathrm{mm}^{3}$ for volume, and number $/ \mathrm{mm}^{3}$ for cell density. Using the additive effect value, the effect size of a QTL and the percentage of variance attributed to a QTL are approximated.

Correlative analysis with behavioral phenotypes and gene transcript expression The GN analytical tools are integrated with data sets of numerous BXD behavioral and physiological phenotypes, and microarray data of gene expression in the brain (Chesler et al., 2003; Chesler et al., 2005), and this facilitates correlative analyses across a wide range of data sets. Transcript expression levels were treated as complex traits and their covariance with the BLA neuroanatomical traits analyzed. Both Pearson's productmoment and Spearman's rank correlation can be computed. For this study, we calculated the Pearson's product-moment correlation between the BLA traits and the mRNA expression level data "INIA Brain mRNA M430 (Jan06)". We also referred to the in situ gene expression data provided by Allen Brain Atlas (ABA) (http://www.brain-map.org) to check for the expression levels of the candidate genes within the BLA. Correlation analysis was also done with BXD neuroanatomical and behavioral traits.

\subsection{Results}

\subsubsection{Volume of the BLA Complex}

The BXD strains exhibit a wide range of variation in the volume of the BLA. BXD19 has the largest mean volume at $1.8 \pm 0.08 \mathrm{~mm}^{3}$ and BXD29 has the smallest at $1.07 \pm 0.03 \mathrm{~mm}^{3}$ (Table 2-1). This amounts to a 1.7-fold difference in size. However, there is no significant difference between the parental strains (B6 at $1.628 \pm 0.1 \mathrm{~mm}^{3}, \mathrm{D} 2$ at $\left.1.55 \pm 0.06 \mathrm{~mm}^{3}\right)$. Relatively small phenotypic difference between parental strains while the progeny RI strains display a wider range of variation is attributed to random assortment of multiple alleles at different loci (Neumann et al., 1993). Depending on the combination of alleles at multiple loci inherited from the parents, a phenotype may be either enhanced or diminished in the offspring. Approximately $35 \%$ of the variance in BLA volume is due to strain genotype $\left(\mathrm{F}_{(37,198)}=2.16, p=0.0005\right)$.

F1 hybrids have the largest BLA and this is consistent with the F1s possessing larger traits for the lateral geniculate nucleus (LGN) (Seecharan et al., 2003), olfactory bulb (Williams et al., 2001a), body weight, and brain weight. More robust traits in the F1s can be explained by positive heterosis and hybrid-vigor (Falconer \& Mackay, 1996).

While the size of the BLA may be modulated by specific genetic factors, the BLA may also be under the influence of genes that have more widespread effects. It is possible that variation in its size could partially be accounted for by variations in the overall brain 
Table 2-1 Basolateral amygdala volume and cell number

\begin{tabular}{lllllllll}
\hline Group & $\mathbf{N}$ & $\begin{array}{c}\mathbf{V}^{\mathbf{A}} \\
\left(\mathbf{m m}^{\mathbf{3}}\right)\end{array}$ & $\begin{array}{c}\mathbf{V}^{\mathbf{B}} \\
\left(\mathbf{m m}^{\mathbf{3}}\right)\end{array}$ & $\begin{array}{c}\mathbf{V}^{\mathbf{C}} \\
\left(\mathbf{m m}^{\mathbf{3}}\right)\end{array}$ & $\mathbf{N}^{\mathbf{D}}$ & $\begin{array}{c}\mathbf{N e u r} \\
(\times \mathbf{K} \mathbf{)}\end{array}$ & $\begin{array}{c}\text { Gli } \\
(\times \mathbf{1 K})\end{array}$ & $\begin{array}{c}\text { Endl } \\
(\times \mathbf{K})\end{array}$ \\
\hline C57BL/6J & 6 & 1.63 & 1.49 & 1.61 & 5 & 119 & 17 & 19 \\
DBA/2J & 6 & 1.55 & 1.63 & 1.56 & 5 & 116 & 14 & 14 \\
BXD1 & 6 & 1.57 & 1.46 & 1.58 & 5 & 146 & 25 & 23 \\
BXD2 & 5 & 1.43 & 1.4 & 1.39 & 5 & 131 & 16 & 14 \\
BXD5 & 6 & 1.60 & 1.36 & 1.6 & 4 & 129 & 16 & 18 \\
BXD6 & 5 & 1.22 & 1.32 & 1.26 & 4 & 88 & 7 & 15 \\
BXD8 & 5 & 1.51 & 1.51 & 1.5 & 5 & 134 & 10 & 11 \\
BXD9 & 5 & 1.51 & 1.5 & 1.52 & 3 & 145 & 7 & 15 \\
BXD11 & 5 & 1.48 & 1.47 & 1.5 & 2 & 158 & 26 & 7 \\
BXD12 & 6 & 1.47 & 1.44 & 1.45 & 5 & 169 & 19 & 15 \\
BXD13 & 6 & 1.39 & 1.41 & 1.38 & 6 & 145 & 20 & 9 \\
BXD14 & 5 & 1.49 & 1.44 & 1.44 & 5 & 134 & 17 & 14 \\
BXD15 & 5 & 1.42 & 1.35 & 1.35 & 3 & 152 & 4 & 13 \\
BXD16 & 6 & 1.55 & 1.48 & 1.54 & 5 & 130 & 14 & 11 \\
BXD18 & 6 & 1.48 & 1.5 & 1.48 & 5 & 135 & 9 & 13 \\
BXD19 & 6 & 1.80 & 1.82 & 1.81 & 5 & 151 & 14 & 15 \\
BXD20 & 4 & 1.52 & 1.59 & 1.53 & 4 & 141 & 9 & 19 \\
BXD21 & 4 & 1.45 & 1.45 & 1.45 & 5 & 128 & 11 & 15 \\
BXD22 & 8 & 1.57 & 1.5 & 1.55 & 5 & 126 & 15 & 17 \\
BXD23 & 5 & 1.48 & 1.44 & 1.45 & 5 & 102 & 15 & 13 \\
BXD24 & 5 & 1.52 & 1.55 & 1.49 & 4 & 140 & 9 & 13 \\
BXD25 & 5 & 1.40 & 1.48 & 1.47 & 5 & 111 & 11 & 21 \\
BXD27 & 5 & 1.43 & 1.6 & 1.43 & 4 & 127 & 11 & 25 \\
BXD28 & 4 & 1.37 & 1.45 & 1.39 & 3 & 117 & 17 & 24 \\
BXD29 & 5 & 1.07 & 1.19 & 1.09 & 4 & 115 & 7 & 25 \\
BXD30 & 5 & 1.14 & 1.34 & 1.19 & 4 & 121 & 9 & 16 \\
BXD31 & 5 & 1.52 & 1.56 & 1.49 & 3 & 169 & 14 & 24 \\
BXD32 & 6 & 1.61 & 1.56 & 1.61 & 6 & 132 & 16 & 22 \\
BXD33 & 5 & 1.32 & 1.34 & 1.35 & 5 & 140 & 15 & 23 \\
BXD34 & 6 & 1.54 & 1.56 & 1.53 & 6 & 148 & 17 & 22 \\
BXD35 & 5 & 1.47 & 1.47 & 1.48 & 5 & 113 & 16 & 24 \\
BXD36 & 6 & 1.44 & 1.49 & 1.48 & 4 & 150 & 17 & 11 \\
BXD37 & 2 & 1.40 & 1.42 & 1.34 & 2 & 117 & 25 & 5 \\
BXD38 & 5 & 1.43 & 1.47 & 1.44 & 3 & 111 & 9 & 15 \\
BXD39 & 5 & 1.34 & 1.4 & 1.38 & 5 & 97 & 12 & 18 \\
BXD40 & 6 & 1.50 & 1.5 & 1.54 & 5 & 117 & 19 & 22 \\
BXD42 & 5 & 1.44 & 1.37 & 1.45 & 4 & 106 & 8 & 23 \\
\hline
\end{tabular}

$\mathrm{N}$ : number of cases per strain for volumetric data; $\mathrm{V}^{\mathrm{A}}$ : raw BLA volume; $\mathrm{V}^{\mathrm{B}}$ : corrected volume after adjusting for brain weight; $\mathrm{V}^{\mathrm{C}}$ : corrected volume after adjusting for body weight; $\mathrm{N}^{\mathrm{D}}$ : number of cases per strain for stereology; Neur: number of neurons; Gli: number of glial cells; Endl: number of endothelial/other cells. 
weight, body weight, age, or sex. The degree of covariance of the BLA volume with these biological factors was evaluated by regression analyses. Multiple linear regression showed brain weight to be a significant predictor of the BLA volume $\left(\mathrm{t}_{195}=4.96, \mathrm{p} \leq\right.$ $0.0001)$. In contrast, body weight, age, and sex of an animal, and the plane of section, are not significant factors for the BLA volume. However, body weight, when used as a single regression factor, correlates significantly with the BLA volume $\left(\mathrm{t}_{195}=3.1, \mathrm{p}=0.002\right)$ but is estimated to account for only $\sim 5 \%$ of the variance. As a single factor, brain weight accounts for $\sim 17 \%$ of the variance $\left(\mathrm{t}_{197}=6.27, \mathrm{p} \leq 0.0001\right)$. Sex, age, and plane of section continue to be insignificant predictors when used as single regression factors. After adjusting the data for the effects of brain weight, and body weight, the corrected data were used for linkage analyses (Table 2-1).

\subsubsection{BLA Cell Counts}

Neurons account for $80 \%$ of the cell population in the BLA. There is about a 2-fold difference in both the number and density of neurons among the BXD strains. BXD12 has the highest population at 169,200 $\pm 14,300$; BXD6 has the lowest at 88,100 \pm 8000 (Table 2-1). As was true for volume, parental strains do not differ significantly $(118,800 \pm 7,500$ in $\mathrm{B} 6$ versus $115,600 \pm 6,200$ in D2). The number and density of nonneuronal cells vary greatly among BXDs with as much as an 8-fold range in the population of glial cells (lowest for BXD15 at 3,700 $\pm 1,300$ and highest for BXD11 at $26,400 \pm 6,500$ ) and a 5-fold range in the population of endothelial cells (lowest for BXD37 at $5200 \pm 3500$ and highest for BXD27 at 25,200 \pm 6400 ). We suspect that some of this variation must be technical, but there is no reciprocal relation between glial cell and endothelial cell counts expecting if cell identification were a major problem. As expected given its large volume, F1 hybrids possess the highest group means for both glial and endothelial cells (Table 2-1). However, the neuron population does not appear to follow this trend and F1s possess average number of neurons and somewhat lower neuron density. The effect of strain on variability in neuron number is over $40 \%(\mathrm{~F}(37,166)$ $=2.88, p<0.0001)$. Strain also accounts for $30-40 \%$ of the variance in glial cell number $\left(\mathrm{F}_{(37,166)}=1.87, p=0.005\right)$.

For the neuron population, multiple linear regression with brain weight, body weight, age, and sex showed none of these factors to be significant predictors. However, regression analysis with body weight as a single factor revealed it to be a statistically significant predictor of the neuron number $\left(\mathrm{t}_{158}=2.34, p=0.02\right)$. Used as single regression factors, brain weight, age, and sex do not show significant covariance with neuron number.

From the regression analyses, we note a great deal of similarity between the BLA volume and glial cell traits. As in the case of the volume, when multiple linear regression is done using brain weight, body weight, age, and sex as co-factors, only the brain weight serves as a significant predictor of the glial cell number, but body weight becomes a significant predictor of glial cell number when it is used as a single regression factor $\left(\mathrm{t}_{158}\right.$ $=3.28, p=0.0013$ ) and is estimated to account for $6 \%$ of the variance. The brain weight 
is estimated to account for $7 \%$ of variance $\left(\mathrm{t}_{164}=3.52, p=0.0006\right)$. Age and sex are insignificant predictors of the glial cell population. The endothelial cell population appears to be unaffected by variations in brain weight, body weight, age and sex. We note a rather large coefficient of error $(\mathrm{CE}=$ standard error/group mean $)$ in the case of the glial cells (average CE of 0.3) and endothelial cells (average CE of 0.2). This high $\mathrm{CE}$ is suggestive of high error rate in data collection, though there is also the possibility that there may be higher within-strain variation for the glial and endothelial cell populations than for neurons. The CE for both the BLA volume and neuronal population averages at only 0.06 .

\subsubsection{QTL Analysis for BLA Volume}

The genome-wide linkage map for the raw BLA volume revealed a significant linkage interval on mid-Chr 8 (Figure 2-2A). This linkage interval is located between 65 and $75 \mathrm{Mb}$, and has a peak LRS of 14, just above the significant threshold. At this locus, the $D$ allele has the positive additive effect and is estimated to increase the volume by $\sim 10 \%$. Two suggestive linkages are also detected on proximal Chr 1 (between 50 and 60 $\mathrm{Mb}$; LRS 9.0) and mid-proximal Chr 19 (between 22 and $28 \mathrm{Mb}$; LRS 9.2). At each of these suggestive linkage intervals, the $B$ allele increases volume by $\sim 10 \%$.

To assess global effects of brain weight and body weight on the linkage map, the BLA volume was regressed against these two factors and the adjusted data were used for interval mapping. Differences in QTLs between the adjusted and raw data would indicate the level of specificity of a particular QTL for the BLA. Adjusting for brain weight eliminates the suggestive linkages on Chr 1 and Chr 19 (Figure 2-2B). The linkage to Chr 8, though weakened, persists at a suggestive LRS of 10.7. The location of the linkage interval is unchanged (between $65-75 \mathrm{Mb}$ ) and the $\mathrm{D}$ allele continues to have the positive additive effect. Regression against body weight also decreases the linkages to Chr 1 and 19, but augments the linkage to mid-Chr 8 to an LRS of 15 (Figure 2-2C). Thus the linkage to mid-Chr 8 is consistently detected on all the linkage maps for the BLA volume, and the significant strength of association (genome-wide $\mathrm{p} \leq 0.05$ ) qualifies it as a QTL for the BLA volume. We will refer to this QTL as Vol8a. However, the reduction in its strength upon adjusting the data for the effect of brain weight indicates a pleiotropic interaction of Vol8a with the size of the BLA and the overall brain weight. Vol8a lies only $\sim 10 \mathrm{Mb}$ proximal to the Cerebellar size $8 a(C b s 8 a$ at $45 \mathrm{cM})$ QTL (Airey et al., 2001).

Simple interval mapping with the stereological data yielded only suggestive QTLs for the neuron (Figure 2-3A) and glial cell populations (Figure 2-3B). No linkage interval was uncovered for the endothelial cells. Both the neuron and glial cell densities share suggestive linkages to mid-proximal Chr 13. Though the overlap in not precise, the linkage intervals for both the neuron and glial traits reside at close proximity, the suggestive LRS peaking at 11.4 for the neuron density and at 10.2 for the glial density. It is noted that this linkage interval is also close to the hippocampal volume QTL, HipV13a (Peirce et al., 2003). At this locus, it is the $D$ allele with the positive additive effect for 

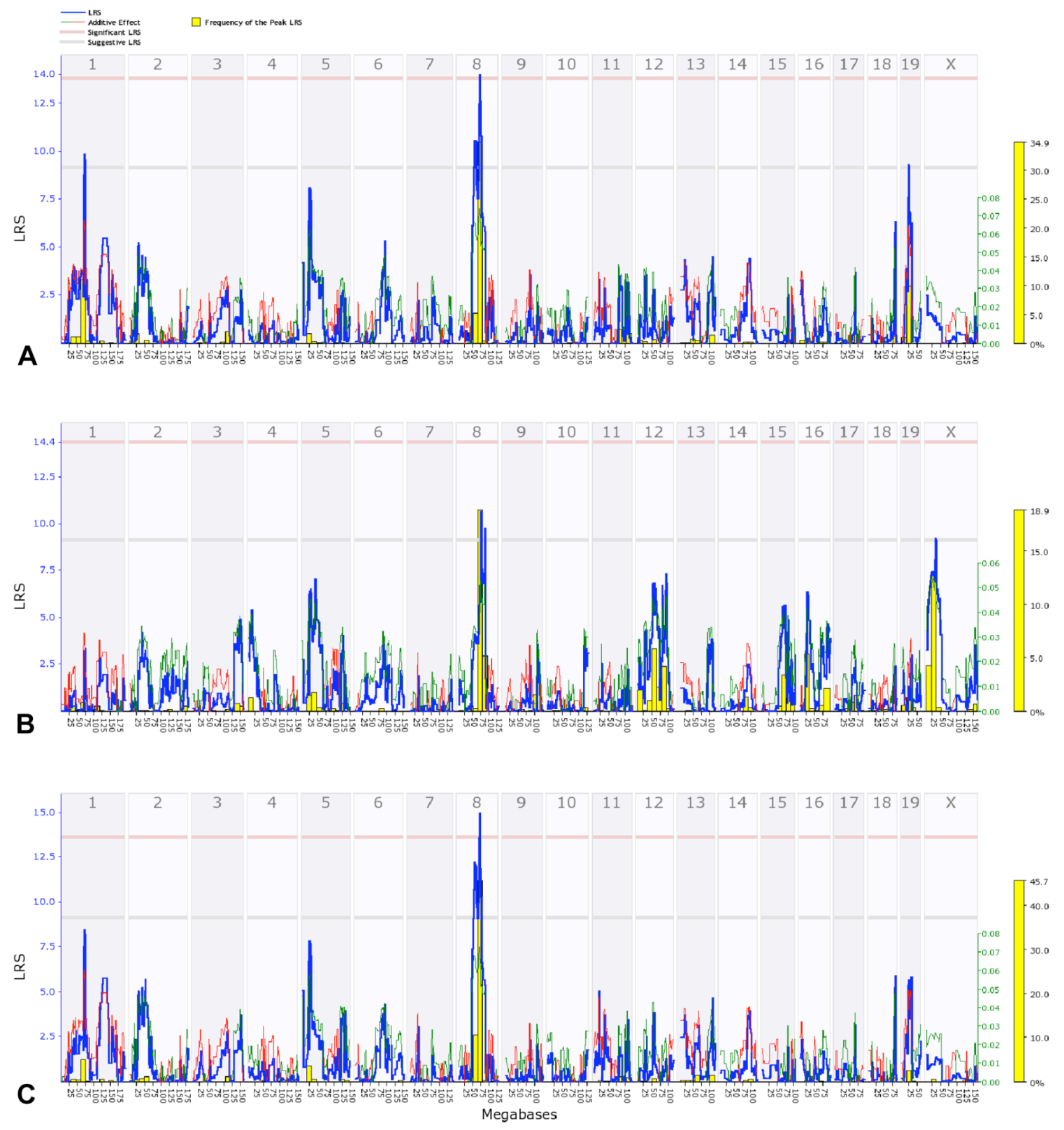

\section{Figure 2-2 QTL analysis of BLA volume}

(A) BLA volume has a significant QTL on Chr 8 (LRS 14) and suggestive peaks on Chrs 1 (LRS 9) and 19 (LRS 9.2). (B) Adjusting BLA volume for brain weight reduces QTL on Chr 8 but linkage remains above suggestive threshold (LRS 10.7). (C) Adjusting BLAc volume for effect of body weight increases QTL on Chr 8 (LRS 15). Blue plot: LRS; green plot indicates positive additive effect for $D$ allele and red plot indicates positive additive effect for $B$ allele; yellow histogram: frequency of peak LRS; pink horizontal line: significant LRS threshold at genome-wide $p \leq 0.05$; grey horizontal line: suggestive LRS threshold at genome-wide $p \leq 0.63$. 

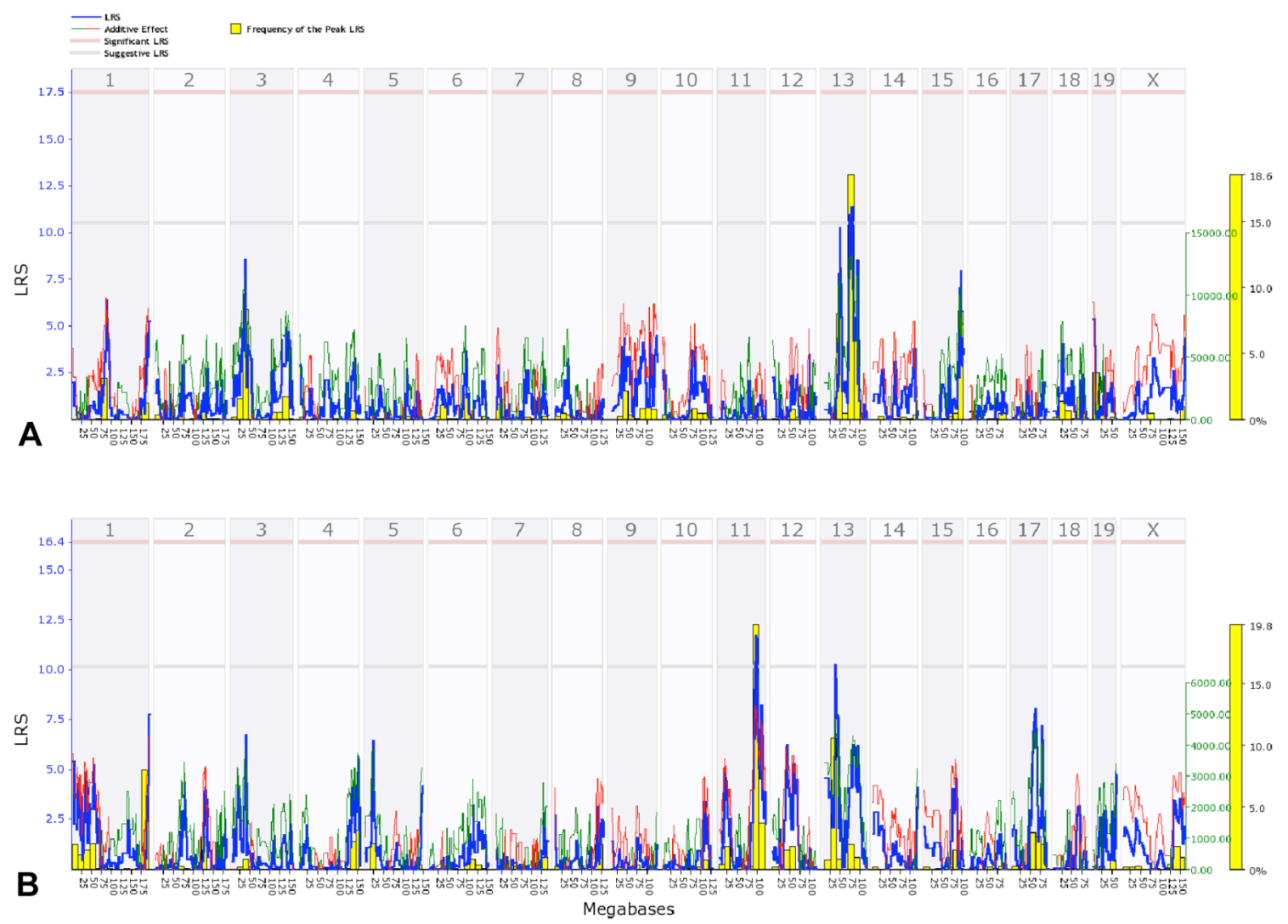

Figure 2-3 QTL analysis of BLA cell counts

(A) BLA neuron density has a suggestive linkage on Chr 13 (LRS 11.4). (B) Glial cell density in the BLA has suggestive linkages on Chrs 11 (LRS 11.4) and 13 (LRS 10.3). 
both neuron and glial cell densities and is estimated to increase the neuron density by $12 \%$ and the glial density by more than $35 \%$.

Another suggestive association is observed for the density of glial cells on distal Chr 11. The suggestive LRS peaks at $\sim 11.4$ between 90 and $105 \mathrm{Mb}$. Glial density, after correction for the effect of brain weight, maps to this locus at a nearly significant LRS of 14.8. The LRS peaks almost precisely over the NeuroD2 gene located at $\sim 98 \mathrm{Mb}$. The B allele at this locus is estimated to increase the glial cell density by more than $25 \%$. Other then the effect of increasing the peak over NeuroD2, regression of the cell counts data against brain weight and body weight do not cause major alterations in the linkage maps.

\subsubsection{QTL Specificity}

Brain weight and body weight are the two most significant predictors of the BLA structure. A genome wide linkage analysis was performed for brain weight (Figure 2-4A) and body weight (Figure 2-4B) to identify any genetic loci that may primarily modulate these global traits but are also linked with the size or cell populations of a specific brain structure such as the BLA. This is a cautionary step to avoid erroneous identification of a more general QTL with widespread effects as being specific for the BLA.

The brain weight variation is associated with a suggestive QTL on Chr 19. For the body weight, suggestive linkages occur on Chrs 7, 10, and 19. This indicates the linkage interval on Chr 19 to primarily modulate gross structures, with a non-specific association with the BLA. Both brain weight and body weight also have linkage peaks of Chr 1, and though both are below the suggestive thresholds, these linkage peaks may account for the suggestive LRS on Chr 1 for the BLA volume that was weakened by regression against brain weight and body weight.

\subsubsection{Correlations between BLA Volume and Cell Populations}

The strongest correlation among the BLA neuroanatomical traits is between its volume and glial number (Figure 2-5A) with a positive Pearson's product correlation $r=$ $0.5, p=0.0007$. Weaker but significant positive correlations exist between the volume and glial cell density (Figure 2-5B; $r=0.4, p=0.03$ ), and volume and neuron number

(Figure 2-5C; $r=0.4, p=0.02$ ). Neuron density in the BLA has the tendency to correlate negatively with volume, and though this does not reach statistical significance (Figure 2-5D; $r=-0.3, p=0.1$, the negative association indicates that smaller BLA will have slightly higher neuronal density. As expected, strong positive correlations relate cellular densities with number. This is especially true for the near perfect correlation between the density and number of glial cells (Figure 2-5E; $r=0.9$ ). Correlation between the number and density of neurons is also highly significant but less stringent compared to glial cells (Figure 2-5F; $r=0.7, p=1.2 \times 10-6$ ). 

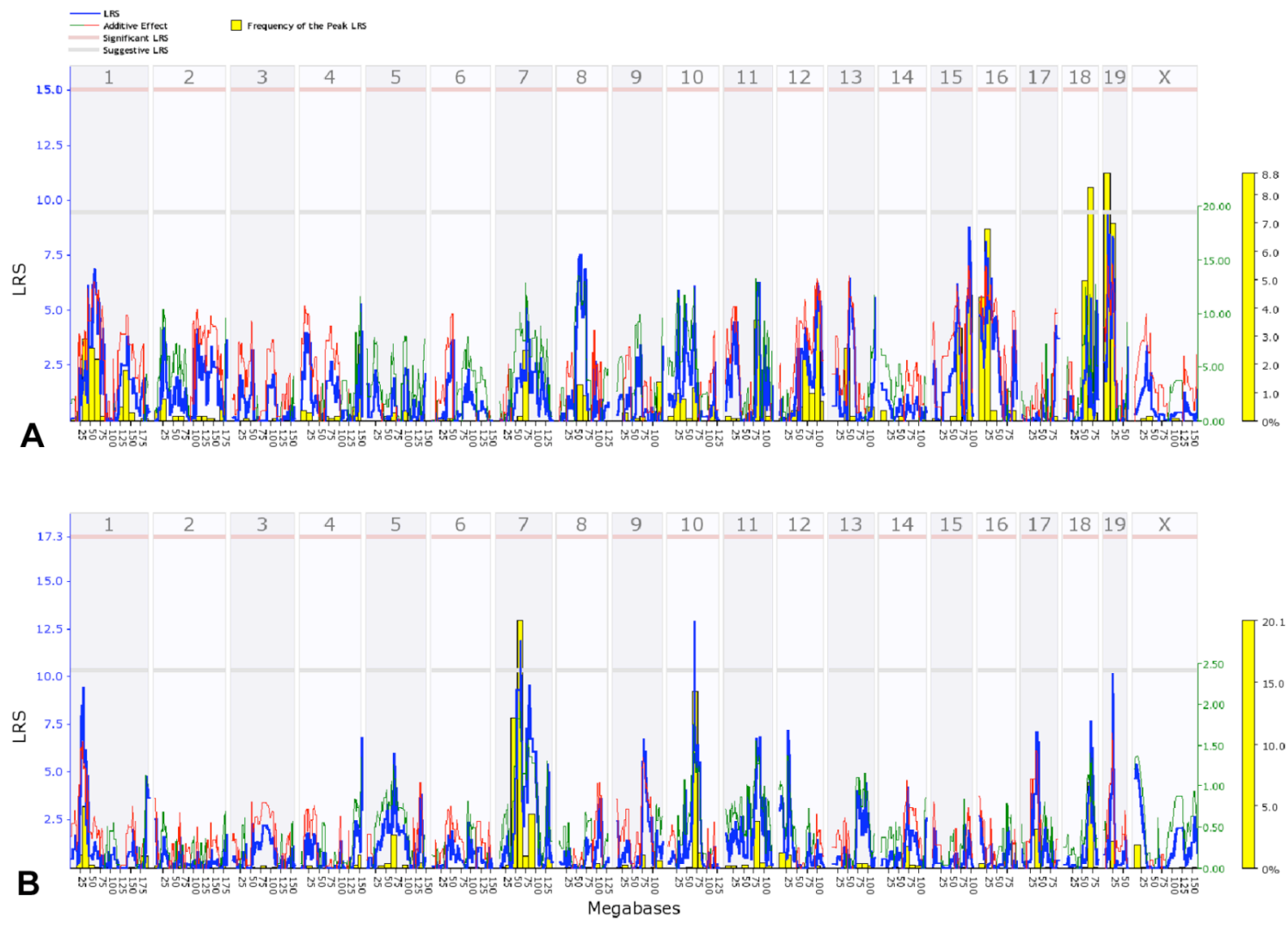

Figure 2-4 QTL maps of brain and body weight

Genome-wide linkage map for (A) brain weight: suggestive linkage on Chr 19; and (B) body weight: suggestive linkages on Chrs 7, 8, 19. The linkage on Chr 19 overlaps with the suggestive linkage for the BLA volume on Chr 19. 


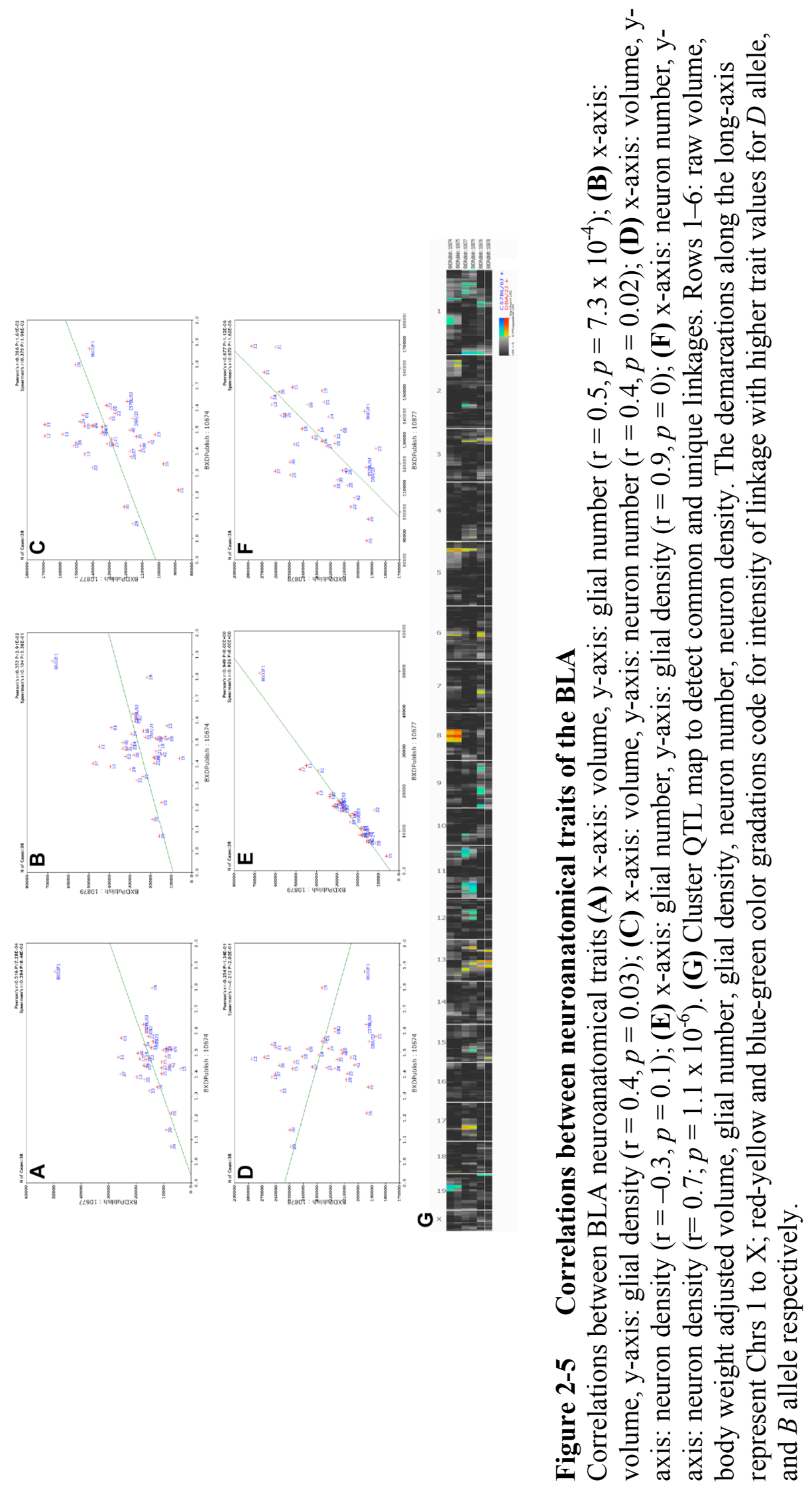


To provide an overview of linkage intervals that may be common for the BLA neuroanatomical traits, a cluster map was generated for, from rows 1 to 6 , the raw volume, body weight adjusted volume, glial number, glial density, neuron number, and neuron density (Figure 2-5G). In addition to the overlapping linkage interval of Chr 13 for the neuron and glial cell populations, closely aligned linkage intervals can be detected on Chr 1 for all traits, on Chr 3 for the neurons and glial cells, on Chr 5 for the volume and glial cells, and on Chr 6 for the volume and neuron number. These linkages are below the significant threshold but may indicate potential shared QTLs.

The complex trait analysis of the BLA uncovered only one significant QTL, Vol8a, associated with its volume, and two suggestive linkage intervals on Chr 11 and Chr 13 associated with the cellular traits.

\subsubsection{Neuroanatomical Correlates of BLA and Comparative QTL Analysis}

GN maintains an archive of diverse phenotypes (BXD Published Phenotypes database) previously collected from the BXD strains by different researchers. This makes it possible to search for traits with significant covariance with the BLA. The "Trait Correlation" tool was use to compute correlations between the BLA structural traits and a variety of other BXD phenotypes that range from physiology, neuroanatomy of other brain regions, to behavior. This analysis showed that the top 15 strongest correlates are all neuroanatomical traits. The strongest association is between the BLA volume and the volume of the hippocampus proper (Figure 2-6A; $\mathrm{r}=0.7, p=4.5 \times 10-6, \mathrm{~N}=34$ ) and its sub-regions such as the dentate gyrus $(\mathrm{r}=0.5, p=0.001)$ and pyramidal cell layer $(\mathrm{r}=$ $0.5, p=0.003$ ). The volume of the BLA also correlates significantly with the sizes of the striatum (Figure 2-6B; $\mathrm{r}=0.6, p=1.2 \times 10-5, \mathrm{~N}=36$ ), and the lateral geniculate nucleus (Figure 2-6C; $\mathrm{r}=0.5, p=4.5 \times 10-3, \mathrm{~N}=35$ ).

To determine if the linkage intervals identified in this study are specific for the BLA, or if common QTLs may be involved in modulating the sizes of different brain structures, we retrieved the volumetric data for the cerebellum and internal granular layer (IGL) (Airey et al., 2001), total hippocampus, hippocampus proper, dentate gyrus and pyramidal cell layer of the hippocampus (Lu et al., 2001; Peirce et al., 2003), LGN (Seecharan et al., 2003), striatum (Rosen et al., 2009), and olfactory bulb weight (Williams et al., 2001a), and performed multiple QTL mapping for these traits. To display the overlapping linkage intervals, a cluster QTL map was generated for, from rows 1 to 10, the IGL, IGL adjusted for brain weight, striatum, hippocampus proper, dentate gyrus, BLA, LGN, olfactory bulb weight, BLA neuron density, and BLA glial density (Figure 2-6D). This analysis confirms Vol8a as a major neuroanatomical QTL but makes it less specific for the BLA. Vol8a is linked to the volumes of the IGL and the whole cerebellum, striatum, and hippocampus proper, but not with the volumes of the dentate gyrus, and LGN. A hint of linkage is also visible between the olfactory bulb weight and Vol8a. In all these cases, it is the $D$ allele in Vol8a that has the positive additive effect. The $C b s 8 a$ QTL reported previously (45 cM; Airey et al., 2001) lies only 


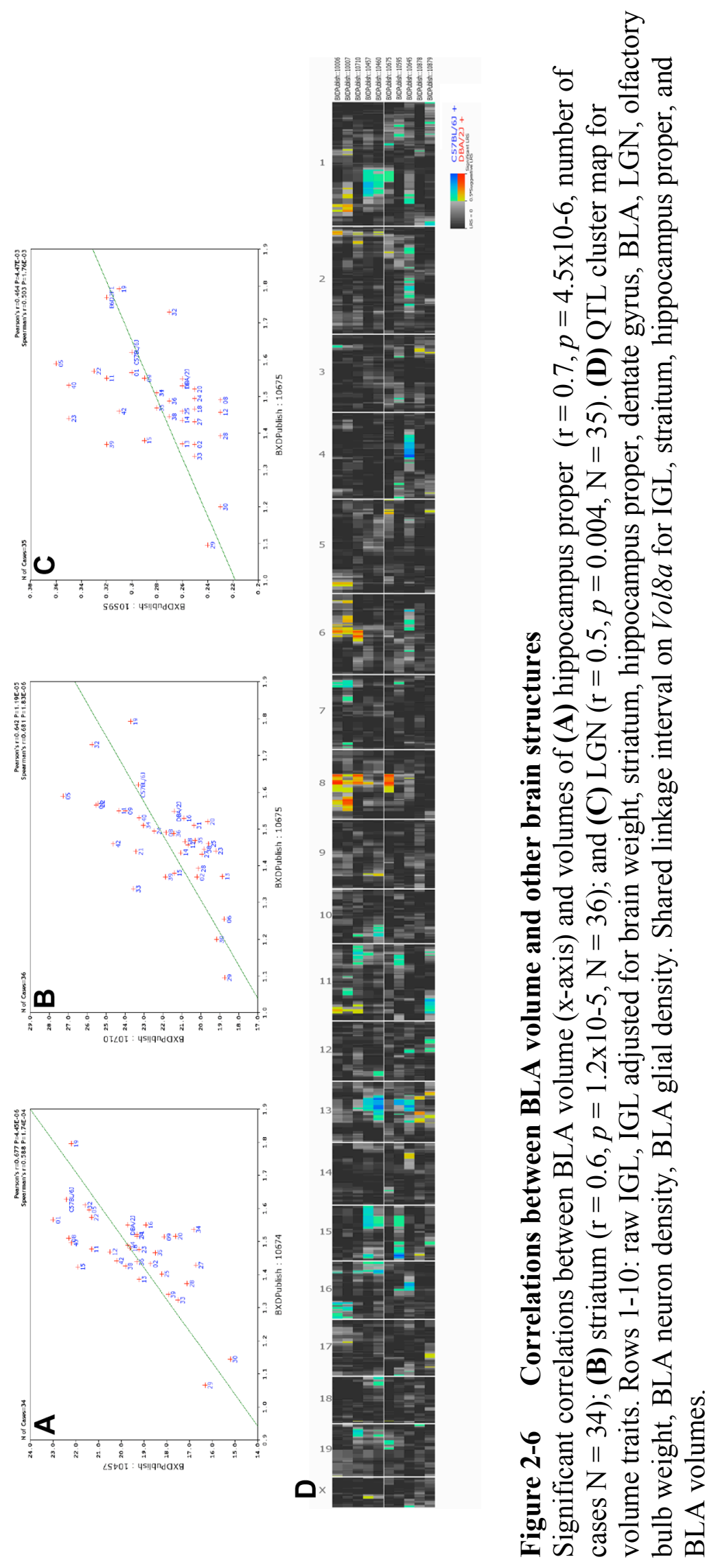


slightly distal to Vol8a. After the cerebellum and IGL volumes are adjusted for brain weight, their linkage interval is shifted from Vol8a to Cbs8a. It is uncertain if Vol8a and $C b s 8 a$ are two separate QTLs with $C b s 8 a$ specific for the cerebellum, or if they represent the same QTL but appear separate due to low mapping resolution. If the later is the case, then there is consensus for the linkage interval to be located on Vol8a. Vol8a may be selectively modulating the sizes of more than one brain structure but not of other brain structures such as the LGN and the dentate gyrus.

\subsubsection{Behavioral Correlates of BLA and Comparative QTL Analysis}

After the neuroanatomical traits, another category of phenotypes that show significant covariance with the BLA structure is behavioral phenotypes related to addiction, and locomotor activity (Table 2-2; Figure 2-7A). Among these, cocaine open field center time behavior (GN trait ID 10333), which assesses the effect of an addictive substance on a behavioral measure of locomotor activity and emotionality, shows the most significant correlation with the BLA volume, and the same trait also correlates significantly with the neuron number $(\mathrm{r}=-0.5, \mathrm{p}=0.02, \mathrm{~N}=23)$. To search for possible common QTLs shared between the BLA and its correlated behavioral phenotypes, a cluster QTL analysis was performed and the most striking overlap of QTLs was found between the BLA volume and the cocaine open field behavior. Another correlated behavior that shares a linkage interval on Vol8a is the locomotor behavioral response measured by Palmer et al. (2002; GN trait ID 10453). A multiple QTL map was generated for these three traits (Figure 2-7B; red: cocaine open field, green: locomotor response, blue: BLA volume). Three overlapping linkage peaks are observed for BLA volume and cocaine open field behavior: (1) though the LRS is only of suggestive strength, the cocaine open field behavior has a linkage overlapping Vol8a, (2) significant peak for the behavioral trait on mid-proximal Chr 1 overlaps the peak that was observed for the BLA traits on Chr 1, (3) though below the suggestive threshold for both traits, another shared peak in LRS is observed on proximal $\mathrm{Chr} 5$. Another region of overlap, but with a much weaker LRS is observed on the distal end of Chr 18. A peak on Volsa is also present for the trait 10453, which measures the effect of 2-hydroxypropyl-betacyclodextrin on locomotor activity (Palmer et al., 2002).

Other significant correlates of the BLA volume are: distance traveled in open field $(\mathrm{r}=0.6, p=0.003, \mathrm{~N}=17$; trait ID 10843; Yang et al., 2008); conditioned place preference ( $\mathrm{r}=-0.6, p=0.003, \mathrm{~N}=22$; GN trait ID 10093, Cunningham, 1995); baseline locomotor activity in grid test $(\mathrm{r}=-0.5, p=0.02, \mathrm{~N}=26$; GN trait ID 10503, Phillips et al., 1996); saccharin preference $(\mathrm{r}=-0.5, p=0.02, \mathrm{~N}=22$; GN trait ID 10550, Risinger et al., 1998); pentylenetetrazol induced seizure $(\mathrm{r}=-0.5, p=0.02, \mathrm{~N}=24$; GN trait ID 10614, Wakana et al., 2000); ethanol induced locomotor response $(\mathrm{r}=0.4, p=0.02, \mathrm{~N}=$ 28; GN trait ID 10790; Demarest et al., 2001); ethanol intake $(\mathrm{r}=0.5, p=0.03, \mathrm{~N}=19$; GN trait ID 10074, Crabbe et al., 1983). 
Table 2-2 Behavioral correlates of BLA

\begin{tabular}{|c|c|c|c|c|}
\hline $\begin{array}{l}\text { X-Axis } \\
\text { BLAc Traits }\end{array}$ & $\begin{array}{l}\text { Y-Axis } \\
\text { Behavioral Traits }\end{array}$ & $\mathbf{r}$ & $p$ & $\mathbf{N}$ \\
\hline \multicolumn{5}{|l|}{ Volume } \\
\hline 10674 & $\begin{array}{l}\text { 10333: Cocaine open filed-center time } \\
\text { (Jones et al., 1999) }\end{array}$ & -0.6 & 0.001 & 23 \\
\hline 10674 & $\begin{array}{l}\text { 10095: Locomotor activity (Cunningham, } \\
\text { 1995) }\end{array}$ & -0.6 & 0.002 & 22 \\
\hline 10674 & $\begin{array}{l}\text { 10490: Cocaine induced sensitization of } \\
\text { locomotor response (Phillips et al., 1998) }\end{array}$ & 0.5 & 0.01 & 27 \\
\hline 10674 & $\begin{array}{l}\text { 10453: Locomotor response (Palmer et al., } \\
\text { 2002) }\end{array}$ & 0.5 & 0.005 & 26 \\
\hline \multicolumn{5}{|l|}{ Glial cells } \\
\hline 10879 & $\begin{array}{l}\text { 10038: Distance traveled (Bolivar \& } \\
\text { Flaherty, 2003) }\end{array}$ & 0.6 & 0.002 & 27 \\
\hline 10879 & $\begin{array}{l}\text { 10783: Novel food preference (Bolivar \& } \\
\text { Flaherty, } 2004\end{array}$ & -0.5 & 0.009 & 26 \\
\hline
\end{tabular}




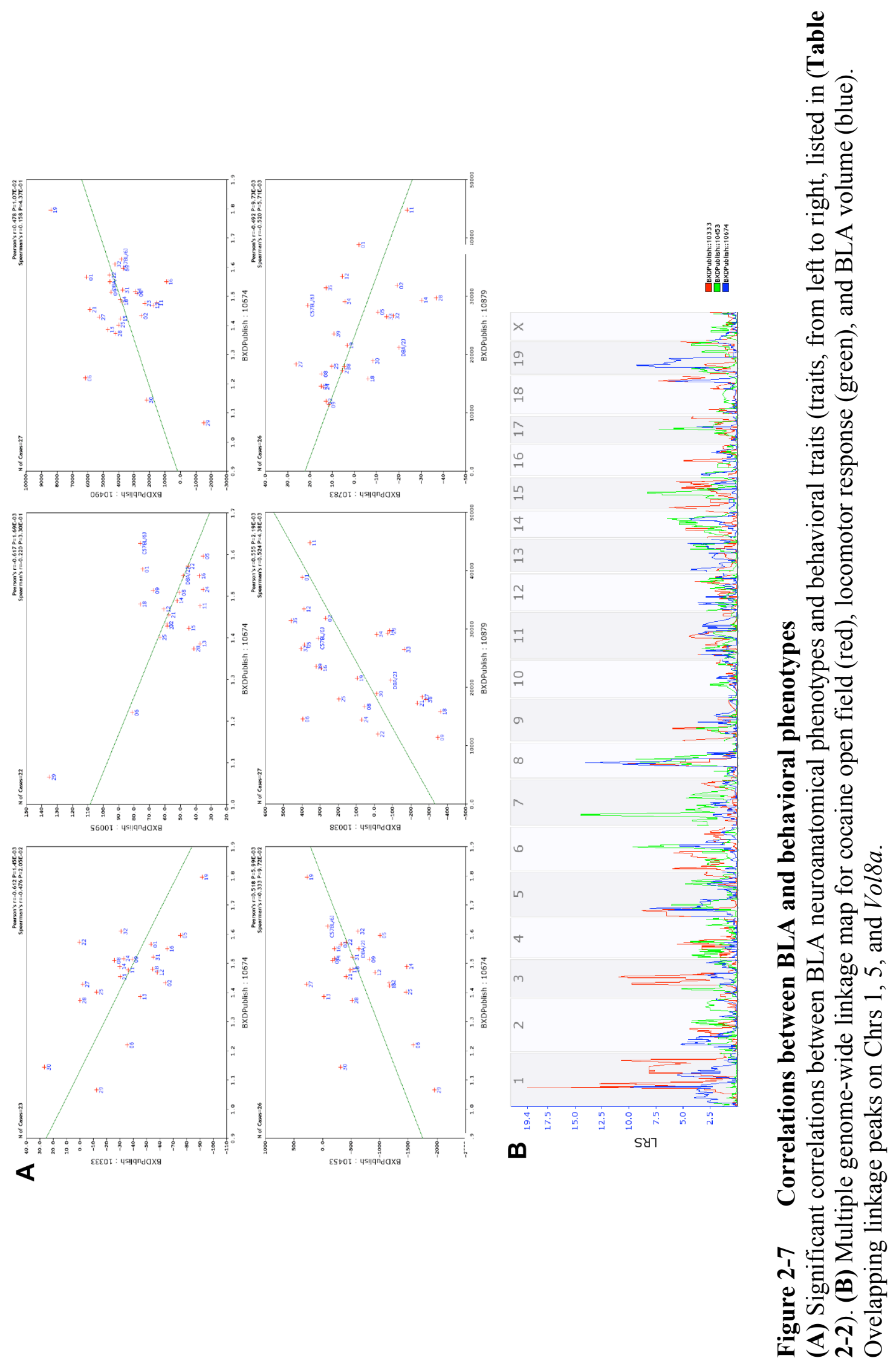




\subsubsection{Candidate Genes}

To narrow the search for candidate genes located within a linkage interval, we selected only those genes that have SNPs between the parental alleles and whose wholebrain expression levels correlate significantly with the BLA traits. Cis-acting genes, which self-regulate their own expression, are considered to be stronger candidates for complex traits as the association between trans-acting genes and complex traits tends to be more complicated (Chesler et al., 2003; Chesler et al., 2005; Kempermann et al., 2006). A criterion for cis-regulated genes is that the genome-wide linkage maps for the expression levels of these genes should have a significant LRS peak within $10 \mathrm{Mbs}$ of its chromosomal location (Chesler et al., 2003; Bystrykh et al., 2005; Chesler et al., 2005).

Several cis-acting genes located in Vol8a correlate with the BLA size and cell counts. Of these, Large (like-glycosyltransferase) shows a very high expression specifically in the BLA (referred to Allen Brain Atlas, Figure 2-1B). It is implicated in the glycosylation of extracellular matrix (ECM) components such and the Large mutant mouse serves as a model for muscular dystrophy and exhibits abnormalities in neuronal migration (Brockington et al., 2005). Other Vol8a candidates include Ssbp4, Zfp617, Sfrs 14, and Fchol. Proteoglycan components of the neural ECM are considered to provide the supporting microenvironment vital for proliferation, migration and differentiation of cells in the nervous system (Oohira et al., 2000; Ida et al., 2005). Chondroitin sulfate proteoglycan 3 (Cspg3) or Neurocan, and Spock3 or Testican3, are two such neural proteoglycan genes located in Volsa. Both Cspg3 and Spock3 are expressed in the BLA (ABA), and though neither are cis-acting genes, they are also considered as possible candidates.

The expression of NeuroD2 (neurogenic differentiation 2) correlates significantly with all aspects of the BLA structure though it is only the glial cell population that maps, at a suggestive LRS, to its locus on Chr 11. The whole-brain expression level of NeuroD2 correlates significantly with the density of neurons $(\mathrm{r}=0.5, p=0.002, \mathrm{~N}=29$; Figure 2-8A), density of glial cells $(\mathrm{r}=0.5, p=0.007, \mathrm{~N}=29$; Figure 2-8B $)$, and volume of BLA $(\mathrm{r}=0.4, p=0.03, \mathrm{~N}=29$; Figure 2-8C). NeuroD2 belongs to the bHLH transcription factor family, and these transcription factors have been implicated in guiding neuron versus glial fate choice of cortical cells (Cai et al., 2000). Specific loss of the BLA has been shown in NeuroD2-null mice and the heterozygotes develop smaller BLA and exhibit aggressive and lowered anxiety behaviors (Lin et al., 2005). The top four transcripts that correlate with BLA volume all belong to $G H$ (Growth Hormone) located on $\mathrm{Chr} 11$ at $\sim 106 \mathrm{Mb}(\mathrm{r}=0.7, p=0.0000009)$. This may be significant as this is only $\sim 8 \mathrm{Mb}$ from NeuroD2. However, NeuroD2 does not appear to be cis-regulated, and other genes located within this interval also correlate with the BLA traits. These include Dlgh4, Thra, Igf2bp and Plxdcl. Based on a higher SNP density and a tendency for cisregulation, Thra (thyroid hormone receptor alpha), which lies within $1 \mathrm{Mb}$ of NeuroD2, is also a prime candidate gene. Thra is strongly expressed in the BLA (ABA) and animals with mutant Thra have been reported to have anxiety, memory, and locomotor impairments (Venero et al., 2005), and abnormal fear and open field behaviors (GuadañoFerraz et al., 2003). 

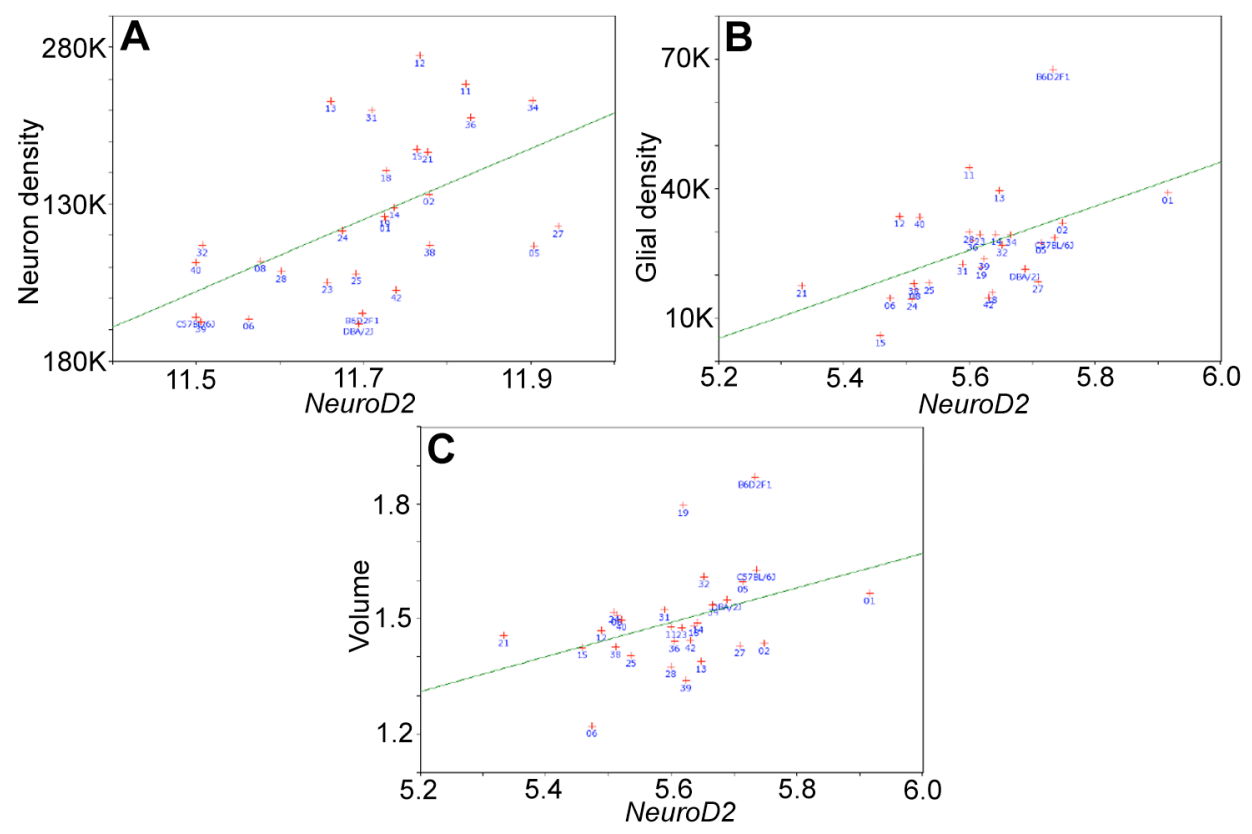

Figure 2-8 Correlations between NeuroD2 and BLA traits

Whole-brain expression levels of NeuroD2 (x-axis) correlates significantly with BLA (A) neuron density (y-axis) $(\mathrm{r}=0.5, p=0.002, \mathrm{~N}=29)$, $(\mathbf{B})$ glial density (y-axis) $(\mathrm{r}=0.5, p=$ $0.007, \mathrm{~N}=29)$, and $(\mathbf{C})$ volume $(\mathrm{r}=0.4, p=0.03, \mathrm{~N}=29)$. 


\subsection{Discussion}

\subsubsection{Summary}

We evaluated the genetic modulation of anatomical variation of the BLA using a diverse panel of strains. Variation for many key parameters among normal strains is often 2-fold or greater. We uncovered a significant QTL on mid-Chr 8 (Vol8a) strongly linked to BLA volume. This region of Chr 8 also contains loci that modulate volume of the cerebellum, striatum, and several hippocampal regions (e.g., the pyramidal cell layer), but not the size of the hippocampal dentate gyrus or the LGN. The most parsimonious explanation is that a single gene variant modulates the size of several regions, but given the size of the Chr 8 QTL interval $(\sim 10 \mathrm{Mb})$ and the large number of candidate genes, this may be a chance colocalization of a set of gene variants with independent mechanisms of affecting the volume of diverse brain regions. A suggestive linkage interval on Chr 13 that may modulate neuron and glial populations in the BLA also overlaps a hippocampal volume QTL (HipV13a).

The most BLA-specific linkage interval is on Chr 11 and it associates specifically with the glial cell population, but the linkage is only suggestive. However, this locus is particularly interesting because it overlaps the chromosomal location of NeuroD2, a gene that is already known to control the BLA development (Lin et al., 2005). Expression of this gene also correlates significantly with the volume of the BLA and its glial and neuronal cell populations.

\subsubsection{Technical Issues}

Estimates of glial cell and endothelial cell numbers have comparatively high errors - CE averaging at 0.3 and 0.2 respectively. While there is the possibility that the higher $\mathrm{CE}$ reflects greater intra-strain variation among isogenic mice, it may also indicate technical limitations in accurate data collection. A possible factor may be the lower average packing density and cell count of the glial and endothelial cell populations relative to the neuron population. The glial and endothelial cells are also considerably smaller in size than the neurons. Despite these differences, we used the same counting criteria for all three cell-types. Thus the volume of tissue through which counts were taken may provide a less adequate representation of the glial and endothelial cell distribution in the BLA because fewer of these cells would be included, and this may have resulted in higher sampling variance. A count box of larger dimensions would have reduced the variance observed in these data (Rosen \& Williams, 2001).

Another factor that may have contributed to the high CE is the low sample sizes from which the cell counts were collected. Adding more subjects would have provided a better representation of a strain's average trait and have lowered the error rate. We used only the coronal sections for stereological measurements as the cell counting process required a more discrete identification of the sub-nuclear regions within the BLA and this 
proved to be a difficult task with the horizontal sections. Despite the low sample size, the error rate is low for the neuron data with a CE of only 0.06 . Nevertheless, we consider the volumetric measurements to be the most reliable trait data we have gathered for the BLA, and it is the linkage map and the QTL yielded by this data that we place more confidence on. However, a concern with the volumetric data is the use of both coronal and horizontal sections. Unlike the cell counting process, the volumetric measurements required the identification of the gross outline of the amygdala from multiple serial sections and summing of these areas to obtain the volume, and measurements could be taken from both coronal and horizontal sections. More coronal sections than horizontal sections were used because the BLA structure is more clearly distinguishable in the coronal sections, but for the final data, strain means were calculated from both types of sections. The regression analysis does not indicate the inclusion of horizontal sections to have a significant effect on the volume data.

\subsubsection{Interactions between QTLs}

Are genetic effects on BLA volume and cell populations specific to this region or are they merely secondary effects of variance in brain weight? Variation in volume of specific brain regions may correlate with variation in brain weight. For example, overall BLA volume correlates with brain weight (explained variance of 16\%). At a finer grain of analysis, we can now begin to resolve the sources of this type of covariance. The strongest and most significant BLA volume locus, Vol8a, also modulates the volumes of the cerebellum and hippocampus, but does not appear to modulate total forebrain volume (Beatty \& Laughlin, 2006). This suggests that the covariance between BLA volume and brain weight can actually be decomposed into an amygdala-hippocampus-cerebellum shared QTL.

An interesting point from this analysis is the quantitative effects of the B and D alleles on the size of the BLA. In the case of Vol8a, the D allele has the positive additive effect and contributes to a larger BLA. In contrast, the suggestive linkage on Chr 19 has the $\mathrm{B}$ allele with a positive additive effect for both the BLA size and brain weight. This may be reflected by the larger brain weight and the generally higher BLA trait values in B6 compared to D2 mice (Table 2-1). However, it is worth noting that after regression correction for brain weight, the relative volume of B6 BLA is actually smaller than that of $\mathrm{D} 2$, though this difference does not reach statistical significance. This trend is consistent with the surprising finding that despite having a much smaller brain than B6, the area of the neocortical barrel field (rostral and dorsolateral neocortex) of D2 is significantly larger than that of B6 (Airey et al., 2005; Li et al., 2005).

Linkage of variation in glial cell density to Chr 11 appears to be more BLA specific. In contrast to most other traits, regression of this trait against brain weight strengthens the LRS. 


\subsubsection{Relations with Other CNS Structures}

The amygdala and hippocampus are two closely associated limbic structures and the covariance in their sizes and possible common genetic linkages is not unexpected. However, the most intense linkage of Vol8a is with the sizes of the BLA and the raw IGL, and Vol8a lies only slightly proximal of Cbs8a (Airey et al., 2002). Although the cerebellum is not a limbic structure, it has been attributed with emotion-related cognitive functions and shares connections with the limbic system (Lee et al., 2004). In addition, both the amygdala and cerebellum have been implicated in the etiology of autism (Abell et al., 1999). Interestingly, in a study on the coordinated evolution of functionally and neuroanatomically related brain regions, the amygdala and cerebellum were the two structures that were unexpectedly indicated to have undergone correlated evolutionary changes in size across animal species (Barton \& Harvey, 2000). These observations point to a more intimate relation between the amygdala and cerebellum.

\subsubsection{Implications for Behavioral Phenotypes}

A large literature implicates the amygdala in a range of behavioral processes (McIntyre et al., 2003; McGaugh, 2004), and mediation of behavioral responses to drugs of abuse (Ryabinin et al., 1999; Floyd et al., 2003; Sharpe et al., 2005). Of particular salience to the present findings, Lin et al. (2005) recently found that mutant mice lacking NeuroD2 have reduced cell number and volume of the BLA, but not other amygdala nuclei. These mice also exhibit low levels of anxiety and impaired emotional learning. This provides an example of how genetically-driven abnormalities in BLA volume may translate to disturbances in complex behavioral phenotypes (Rosvold et al., 1954; Prather et al., 2001). In this context, a correlative analysis of BLA traits with a range of behavioral phenotypes catalogued in GN highlights significant associations between BLA and responses to drugs of abuse, and emotionality. The association is especially significant between the BLA volume and cocaine induced behavioral responses as measured by the open field behavior (Jones et al., 1999; GN trait 10333). The shared linkage intervals between the brain structure and behavioral phenotype signify multiple genetic loci that may modulate the formation and structure of a neural substrate, which, in

turn, underlies a complex behavior. Several studies have underscored the role of the BLA in chronic addiction to drugs of abuse and cocaine seeking behavior (See et al., 2003; Fuchs et al., 2006), and psychostimulants which alter locomotor activity levels in animals have been shown to have specific effects on the basolateral complex (Trinh et al., 2003). A genetic dissection of the amygdala may provide further insights into genetics of these behaviors.

\subsubsection{Conclusion}

In this study we combined three complimentary methods to study the BLA: (1) a classic quantitative dissection of the BLA; (2) an analysis of gene expression across the same BXD strains, as well as high resolution spatial data on gene expression using in situ 
gene expression data provided by ABA; and (3) a correlative analysis using 30 years of studies on the BXD strains. On the basis of transcript expression covariance with the BLA size and cell populations, and the presence of SNPs, the following three genes are prime candidates: Large, NeuroD2, and Thra. 


\section{CHAPTER 3. VARIATION IN MOUSE BASOLATERAL AMYGDALA VOLUME IS ASSOCIATED WITH DIFFERENCES IN STRESS REACTIVITY AND FEAR LEARNING*}

\subsection{Synopsis}

A wealth of research identifies the amygdala as a key brain region mediating negative affect, and implicates amygdala dysfunction in the pathophysiology of anxiety disorders. Although there is a strong genetic component to anxiety disorders such as posttraumatic stress disorder (PTSD) there remains debate about whether abnormalities in amygdala function predispose to these disorders. In the present study, groups of C57BL/6 $\mathrm{x} \mathrm{DBA} / 2(\mathrm{~B} \times \mathrm{D})$ recombinant inbred strains of mice were selected for differences in volume of the basolateral amygdala complex (BLA). Strains with relatively small, medium, or large BLA volumes were compared for Pavlovian fear learning and memory, anxiety-related behaviors, depression-related behavior, and glucocorticoid responses to stress. Strains with relatively small BLA exhibited stronger conditioned fear responses to both auditory tone and contextual stimuli, as compared to groups with larger BLA. The small BLA group also showed significantly greater corticosterone responses to stress than the larger BLA groups. BLA volume did not predict clear differences in measures of anxiety-like behavior or depression-related behavior, other than greater locomotor inhibition to novelty in mice with smaller BLA. Neither striatal, hippocampal nor cerebellar volumes correlated significantly with any behavioral measure. The present data demonstrate a phenotype of enhanced fear conditioning and exaggerated glucocorticoid responses to stress associated with small BLA volume. This profile is reminiscent of the increased fear processing and fear reactivity that is associated with amygdala excitability and reduced amygdala volume in humans carrying loss of function polumorphism in the serotonin transporter and monoamine oxidase A genes. Our study provides a unique example of how natural variation in amygdala volume associates with specific fear- and stress-related phenotypes in rodents, and further supports the role of amygdala dysfunction in anxiety disorders such as PTSD.

\subsection{Introduction}

The past decade has seen great advances in understanding how genetically driven individual differences in the anatomy and function of specific brain regions confer risk for a variety of neuropsychiatric diseases (Caspi \& Moffitt, 2006; Hariri et al., 2006). This literature is based upon the assumption that associations between genes, neural function, and clinical end points are causative in nature. However, associations between clinical symptoms and measures of neural activation are invariably made $a$ posteriori:

\footnotetext{
* Adapted with permission from Nature Publishing Group. Yang RJ, Mozhui K, Karlsson RM, Cameron HA, Williams RW, Holmes A (2008). Variation in mouse basolateral amygdala volume is associated with differences in stress reactivity and fear learning. Neurophychopharmacology 22:2595-2604.
} 
that is, after the phenotype is observed. By contrast, there has been little demonstration that variation in a brain region of interest can successfully predict, a priori, the likely prevalence of a clinical or behavioral trait.

A pertinent case in point is posttraumatic stress disorder (PTSD). There is a significant genetic component to risk for PTSD (Kendler, 2001), as well as neurological abnormalities in unaffected probands that are suggestive of antecedent genetic or familial risk factors (Gurvits et al., 2006). Functional neuroimaging studies have identified hyperactivityof the amygdala as a neural correlate for the clinical symptoms seen in PTSD (Rauch et al., 2006; Shin et al., 2006). In addition, some but not all structural imaging and postmortem studies have found a trend for smaller left amygdala volume in patients with the disorder (Bremner et al., 1997; Bonne et al., 2001; De Bellis et al., 2001, 2002; Fennema-Notestine et al., 2002; Lindauer et al., 2004; Wignall et al., 2004; Karl et al., 2006). Taken together, these findings suggest that amygdala dysfunction may predispose individuals to PTSD and other stress-related disorders. However, while there are data showing that smaller hippocampal volume can precede the development of PTSD (Gilbertsob et al., 2002), the hypthesis that variation in amygdala anatomy can lead to PTSD has not been directly tested.

A major role for the amygdala in PTSD is consistent with a large corpus of data from animal models demonstrating amygdala mediation of a spectrum of behavioral and mnemonic functions that include, but are not limited to, the regulation of emotion and negative affect (Davis \& Whalen, 2001; Robbins \& Everitt, 2002; Fanselow \& Poulos, 2005; Phelps \& LeDoux, 2005). Ablation of specific subnuclei of the rat amygdala produces alterations in certain fear-, anxiety-, and stress-related behaviors. Specifically, the basolateral amygdala complex (BLA) appears necessary for the formation and/or expression of associative fear memories. This has led to a model in which the lateral nucleus within BLA serves as a convergence site for sensory and aversive information that is relayed eitherdirectly or via the basal nucleus to the major output center of the amygdala, the central nucleus (CeA), to drive fear-related behaviors (Fanselow \& LeDoux, 1999; Maren \& Quirk, 2004). Thus, rodents receiving pre-training lesions of the lateral, but not basal, nucleus fail to form fear memories (Goosens \& Maren, 2001; Nader et al., 2001; Koo et al., 2004), while post-training lesions of the basal nucleus prevent the expression of fear memory but not the ability to acquire new memories (AngladaFigueroa \& Quirk, 2005). These findings provide clear evidence that the BLA is necessary for certain forms of learned fear behavior. They do not, however, address the issue of whether natural variation in amygdala anatomy and function is sufficient to predict differences in these behaviors.

In the present study, we sought to address this issue by utilizing a genetically defined panel of C57BL/6 x DBA/2 (BXD) recombinant inbred strains that we have recently shown to exhibit up to 2-fold variation in BLA volume in large sample of $37 \mathrm{RI}$ lines (Mozhui et al., 2007). BXD RI mice are inbred strains derived from C57BL/6 and DBA/2 (Taylor, 1989; Chesler et al., 2003) that have been used to identify chromosomal loci underlying fear- and anxiety-related phenotypes (Caldarone et al., 1997; Owen et al., 1997; Wehneret et al., 1997; Valentinuzzi et al., 1998), as well as volume and cell 
number variation in various brain regions (Neumannet et al., 1993; Airey et al., 2001, 2002; Lu et al., 2001; Rosen \&Williams, 2001; Williams et al., 2001a; Seecharan et al., 2003). We selected 17 BXD RI lines and assigned them to subgroups with relatively small, medium, and large BLA volume. Our objective was to test whether a priori categorization of mice according to BLA volume differences within a natural range was sufficient to predict differences in fear-, anxiety-, and stress-related phenotypes. The results obtained provide some of the strongest evidence to date that genetically driven variation in amygdala volume is sufficient to predict the magnitude of fear- and stressrelated phenotypes.

\subsection{Materials and Methods}

\subsubsection{Histology}

Histological analysis of the BXD RI lines was performed previously at the Department of Anatomy and Neurobiology, University of Tennessee Health Science Center, and is described in detail by Mozhui et al. (2007). Briefly, the BLA complex was defined as the portion between the external capsule and the amygdalar capsule, as described by Swanson and Petrovich (1998). This includes the lateral and basolateral, but not basomedial, nuclei (Figure 3-1A). Images from bilateral $30 \mathrm{~mm}$ thick serial sections were captured from a Zeiss light microscope to a computer and the BLA was manually traced to determine cross-sectional area $\left(\mathrm{mm}^{2}\right)$ along the entire rostrocaudal thickness of the BLA (B6-9 sections for each brain). Volume was calculated by multiplying the interval thickness between serial sections (which was $300 \mathrm{~mm}$ ) and correcting for volumetric shrinkage caused by the histological process ((measured values/total brain volume) $\mathrm{x}$ expected brain volume for given brain weight). Post-processing total brain volume was determined by the point-counting method described by Williams (2000). These data can be viewed at an anatomical database at http://www.genenetwork.org.

\subsubsection{Selection of BXD RI Lines and Assignment to BLA Volume Groups}

In an earlier study, we conducted a comprehensive analysis of 35 BXD RI lines (plus the C57BL/6J and DBA/2J parental lines) for BLA volume (Mozhui et al., 2007). This revealed up to 2-fold variation in BLA volume across this panel of RI lines. In the current study we obtained a subset of this larger panel. We selected lines on the basis of a desire to obtain a range of relatively small, medium, and large BLA volumes; but this was limited by the availability of lines from a single commercial vendor (The Jackson Laboratory, Bar Harbor, ME), which was necessary to avoid a potentially confounding source of variability (ie supplier). Of the $15 \mathrm{BXD}$ RI lines (plus the C57BL/6J and DBA/2J parental lines) obtained, we cross-referenced with the dataset (Mozhui et al., 2007) to assign an approximately equal number of lines to 'small,' 'medium,' and 'large' groups. This resulted in five lines in the small group (BXD13, BXD21, BXD28, BXD38, 

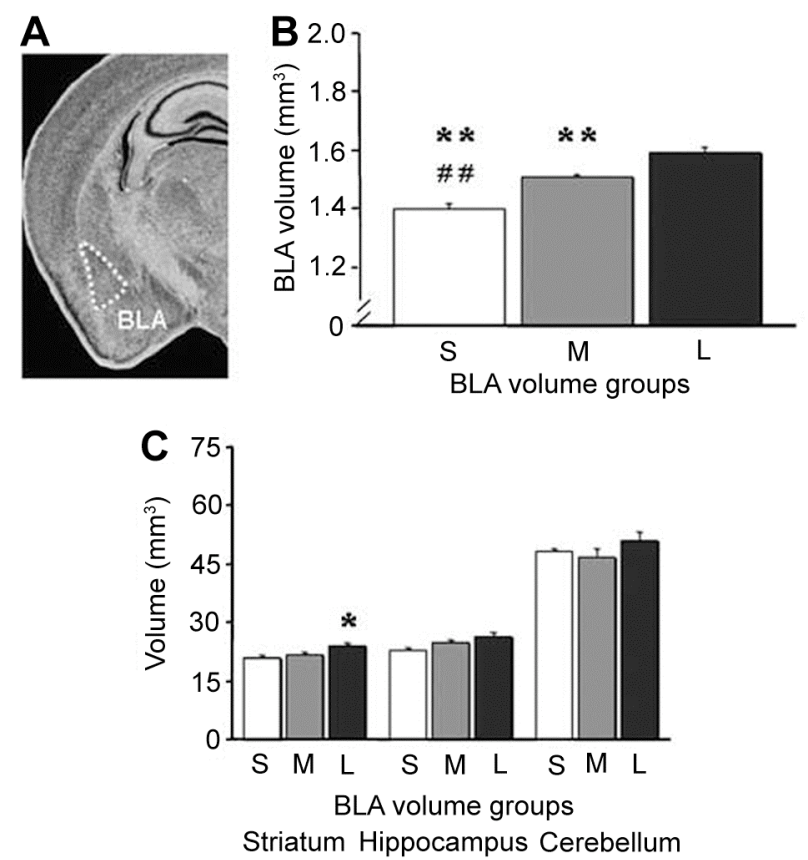

Figure 3-1 Basolateral amygdala volume differences in BXD RI lines

(A) Neuroantomical definitions of the BLA complex. (B) BLA volume differences in mice selected for relatively small (S), medium (M), and large (L) BLA. (C) Dorsal striatal, total hippocampal, and total cerebellar volume differences in mice selected for BLA volume differences ( $\mathrm{n}=24-34$ per volume group). ${ }^{* *} p<0.01 \mathrm{vs} \mathrm{L},{ }^{\# \#} p<$ vs M. 
and BXD39), seven lines in the medium group (BXD8, BXD11, BXD12, BXD23, BXD31, BXD34, and DBA/2J), and five lines in the large group (BXD16, BXD19, BXD22, BXD32, and C57BL/6J). Next, we took the BLA volume data for each line (an average of $n=4-8$ mice per line) from the dataset (Mozhui et al., 2007) and calculated the mean average and within-group variance of BLA volume within each of our three volume groupings. Volume differences between the subgroups were then analyzed using one-way analysis of variance (ANOVA) followed by Newman-Keuls post hoc analysis. This showed that the subgroups differed significantly from one another (main effect of volume group: $\mathrm{F}_{2,14}=18.58$, post hoc comparisons: all $p<0.01$ ). For depiction of mean differences and within-group variance as measured by standard error, see Figure 3-1B.

To determine whether BLA volume differences in our groups generalized to other brain regions, we obtained the volume values for dorsal striatum (caudate putamen), total hippocampus, and total cerebellum in each of the RI lines we tested from http://www.genenetwork.org. These data were originally described in the following sources: for dorsal striatum, Rosen and Williams (2001) and Lu et al. (2001); for hippocampus, including dentate gyrus, excluding subiculum, Peirce et al. (2003); and for cerebellum, Airey et al. (2001). One-way ANOVA and post hoc tests showed that the subset of RI lines we designated as the large BLA groups also had significantly greater striatal volume than the group designated as the small BLA group (main effect of group: $\mathrm{F}_{2,14}=3.78, p<0.05$, post hoc comparison: $p<0.05$; Figure 3-1C). ANOVA showed that the BLA subgroups did not significantly differ in either total hippocampal or cerebellar volume (Figure 3-1C).

\subsubsection{Animal Husbandry and Behavioral Phenotyping}

Mice were bred at The Jackson Laboratory and transported to the NIH at $\sim 8$ weeks of age and housed 1-4 per cage in same-strain groupings in a temperature- and humidity-controlled vivarium under a $12 \mathrm{~h}$ light-dark cycle (lights on 0600 hours). There were a total of 190 male mice at the start of the study, with at least $n=8-12 /$ RI line except for BXD28 $(n=5)$. This resulted in a total of 47 mice in the small BLA group, 82 in the medium BLA group, and 61 in the large BLA group. Behavioral testing was conducted during the light phase between 0900 and 1700 hours. Given evidence of qualitative differences between rodent tests for anxiety-like behavior (Holmes et al., 2003), three separate tests were employed. Mice were tested on a battery of tests in the following order with the putatively more stressful assays at the end of the sequence and at least 1 week between tests: exploratory and anxiety-like behavior (novel open-field, elevated plus-maze, light-dark exploration tests), Pavlovian fear conditioning, depression-related behavior (forced swim test, FST), hot plate (as a control for pain perception in fear conditioning), and glucocorticoid responses to stress (corticosterone levels following swim stress). All experimental procedures were performed in accordance with the National Institutes of Health Guide for Care and Use of Laboratory Animals and were approved by the local Animal Care and Use Committee. 


\subsubsection{Pavlovian Fear Conditioning}

Pavlovian fear conditioning (Kim \& Fanselow, 1992) was conducted in a chamber with transparent walls and a metal rod floor based on methods described previously (Wellman et al., 2007). To provide a distinctive olfactory environment, the chamber was cleaned between subjects with a 79.5\% water/19.5\% ethanol $/ 1 \%$ vanilla extract solution. After an initial $120 \mathrm{~s}$ acclimation period, the mouse received three pairings (60-120 s interval after each pairing) between the conditioned stimulus (CS; $30 \mathrm{~s}, 80 \mathrm{~dB}, 3 \mathrm{kHz}$ tone) and the unconditioned stimulus (US; $2 \mathrm{~s}, 0.6 \mathrm{~mA}$ scrambled footshock), in which the US was presented during the last $2 \mathrm{~s}$ of the CS. The presentation of stimuli was controlled by a San Diego Instruments (San Diego Instruments, San Diego, CA) or Med Associates fear conditioning system (Med Associates, Burlington, VT).

After $24 \mathrm{~h}$, expression of the fear memory for the CS was tested in a novel context, in a different room from training. The novel context had black/white checkered walls and a solid Plexiglas, opaque floor. This chamber was cleaned between subjects with a $50 \%$ ethanol $/ 50 \%$ water solution. After an initial $180 \mathrm{~s}$ acclimation period, the CS was continuously presented for $180 \mathrm{~s}$. After $24 \mathrm{~h}$, expression of the fear memory for the conditioned context was tested in the same room as conditioning by placing the mouse in the training chamber for 5 min. Freezing was defined as absence of any visible movement except that required for respiration, and was scored at $5 \mathrm{~s}$ intervals by an observer who was blind to BXD line. The number of observations scored as freezing was converted to a percentage ((number of freezing observations/total number of observations) x 100) for analysis.

The hot plate test was conducted as a control for group differences in pain perception (Boyce-Rustay \& Holmes, 2006). The apparatus was a flat plate (Columbus Instruments, Columbus, $\mathrm{OH}$ ) heated to $551 \mathrm{C}$ on which the mouse was placed. The latency to show a hind paw shake or lick was timed by an observer, with a maximum response latency of $30 \mathrm{~s}$.

\subsubsection{Depression-Related Behavior and Glucocorticoid Responses to Stress}

The FST was conducted as previously described (Porsolt et al., 1978; Hefner \& Holmes, 2007). The apparatus was a transparent Plexiglas cylinder $(25 \mathrm{~cm}$ high, $20 \mathrm{~cm}$ diameter) filled halfway with water $\left(24 \pm 1^{\circ} \mathrm{C}\right)$ into which the mouse was gently lowered for a 10 min trial. The presence/absence of immobility (cessation of limb movements except minor involuntary movements of the hind limbs) was manually observed using an instantaneous sampling technique every $5 \mathrm{~s}$ during the last $8 \mathrm{~min}$ and expressed as a percentage of total observations. The result was converted to a percentage ((number of immobility observations/total number of observations) x 100) for analysis.

Glucocorticoid responses to stress were measured after a 4-week rest period following the FST described above. The mouse was subjected to a 10 min forced swim stress trial and then returned to the home cage. After $30 \mathrm{~min}$, mice were killed via rapid 
cervical dislocation and decapitation to collect trunk blood. Nonstressed controls were killed within $30 \mathrm{~s}$ of removal from the home cage. To minimize disturbance of nonstressed controls, all mice were individually housed $24 \mathrm{~h}$ prior to killing. Blood samples were centrifuged at 13000 r.p.m. for 30 s. Serum was extracted and assayed for total corticosterone (bound and free) using the Coat-a-Count RIA TKRC1 kit (limit of detection 5.7 ng/ml; Diagnostic Products Corp, Los Angeles) as previously described (Boyce-Rustay et al., 2007).

\subsubsection{Anxiety-Related Behavior}

The novel open-field test was conducted as previously described (Wiedholz et al., 2007). The apparatus was a square arena $(39 \mathrm{~cm} \times 39 \mathrm{~cm} \times 35 \mathrm{~cm}$ ) with opaque white Plexiglas walls and floor that was evenly illuminated to B20 lux. The mouse was placed in a corner and allowed to freely explore for $30 \mathrm{~min}$. Distance traveled, time spent moving, time spent in the $(20 \mathrm{~cm} \times 20 \mathrm{~cm})$ center, and entries into the center were measured using the Ethovision videotracking system (Noldus Information Technology Inc., Leesburg, VA). The apparatus was cleaned with $70 \% \mathrm{EtOH}(\mathrm{v} / \mathrm{v})$ and dried between subjects.

The elevated plus-maze test was conducted as previously described (Handley \& Mithani, 1984; Boyce-Rustay \& Holmes, 2006). The apparatus consisted of two open arms (30 cm x $5 \mathrm{~cm} ; 55 \mathrm{lux})$ and two closed arms (30 cm x $5 \mathrm{~cm}$ x $15 \mathrm{~cm} ; 5 \mathrm{lux})$ extending from a $5 \mathrm{~cm} \times 5 \mathrm{~cm}$ central area and elevated $20 \mathrm{~cm}$ from the ground (San Diego Instruments). The walls were made from black ABS plastic and the floor from white ABS plastic. A $0.5 \mathrm{~cm}$ raised lip around the perimeter of the open arms prevented mice from falling off the maze. The mouse was placed in the center facing an open arm and allowed to explore the apparatus for $5 \mathrm{~min}$. Time spent in the open arms, and entries into the open and closed arms were measured by the Ethovision videotracking system (Noldus Information Technology Inc.). The apparatus was cleaned with $70 \% \mathrm{EtOH}(\mathrm{v} / \mathrm{v})$ and dried between subjects.

The light-dark exploration test was conducted as previously described (Crawley, 1981; Boyce-Rustay \& Holmes, 2006). The apparatus consisted of two compartments (each $17 \mathrm{~cm} \times 13 \mathrm{~cm} \times 13 \mathrm{~cm}$ ), one with white Plexiglas walls and clear Plexiglas floor (40 lux) ('light' compartment) and the other with black Plexiglas walls and clear Plexiglas floor (0 lux) ('dark' compartment), which were connected by a partition at floor level with a small opening $(5 \mathrm{~cm})$ (Med Associates, Georgia, VT, Model ENV-3013). The mouse was placed into the dark compartment facing away from the aperture and allowed to explore the apparatus for $10 \mathrm{~min}$. Time spent and full-body transitions into the light compartment, and total full-body transitions between the light and dark compartments were measured by photocells connected to Med Associates software. The apparatus was cleaned with $70 \% \mathrm{EtOH}(\mathrm{v} / \mathrm{v})$ and dried between subjects. 


\subsubsection{Statistics}

BLA group effects were analyzed using ANOVA and Newman-Keuls post hoc tests where appropriate. One mouse was an extreme outlier (45 standard deviation from the mean) for preconditioning freezing and was excluded from the study. Genetic correlations were performed (treating each of the 17 strains as an individual data point) between BLA, dorsal striatal, total hippocampal, and cerebellar volume, and behavioral and neuroendocrine measures using Spearman's test. The threshold for statistical significance was set at $p<0.05$.

\subsection{Results}

\subsubsection{Pavlovian Fear Conditioning}

There was no significant difference between BLA groups for percent freezing during the (2-min) preconditioning or (2-min) postconditioning periods (Figure 3-2A). BLA groups significantly differed in percent freezing to the $\mathrm{CS}\left(\mathrm{F}_{2,158}=7.74, p<0.01\right)$ and context $\left(\mathrm{F}_{2,159}=5.37, p<0.01\right)$. The small BLA group froze more in response to the CS (Figure 3-2B) and to the context (Figure 3-2C) than the medium or large groups. There was a significant genetic correlation between freezing to CS and volume of BLA, but not striatum, hippocampus, or cerebellum (Table 3-1).

There were no group differences in the hot plate test for nociception (mean \pm SEM latency to respond: small $=6.42 \pm 0.47 \mathrm{~s}$, medium $=7.39 \pm 0.48 \mathrm{~s}$ and large $=6.14$ $\pm 0.37 \mathrm{~s})$.

\subsubsection{Depression-Related Behavior and Glucocorticoid Responses to Stress}

There was no effect of BLA group on percent immobility in the FST (Figure 3-3A). Corticosterone levels were affected by stress in a BLA group-dependent manner (stress $\mathrm{x}$ group interaction: $\mathrm{F}_{2,41}=3.41, p<0.05$ ). While there were no group differences in corticosterone in nonstressed mice, corticosterone levels following stress were significantly greater in the small BLA group than the medium or large groups (Figure 3-3B). There was a significant baseline correlation between nonstressed corticosterone and striatal, but not BLA, hippocampus, or cerebellar, volume (Table 3-1).

\subsubsection{Tests for Anxiety-Related Behavior}

There was a significant effect of BLA group on distance traveled $\left(\mathrm{F}_{2,186}=8.21, p\right.$ $<0.01)$, but not percent time in the center in the novel open-field test. The small BLA volume group traveled less than the medium or large BLA volume groups (Figure 3-4A), while percent time in the center was no different between groups (Figure 3-4B). There 

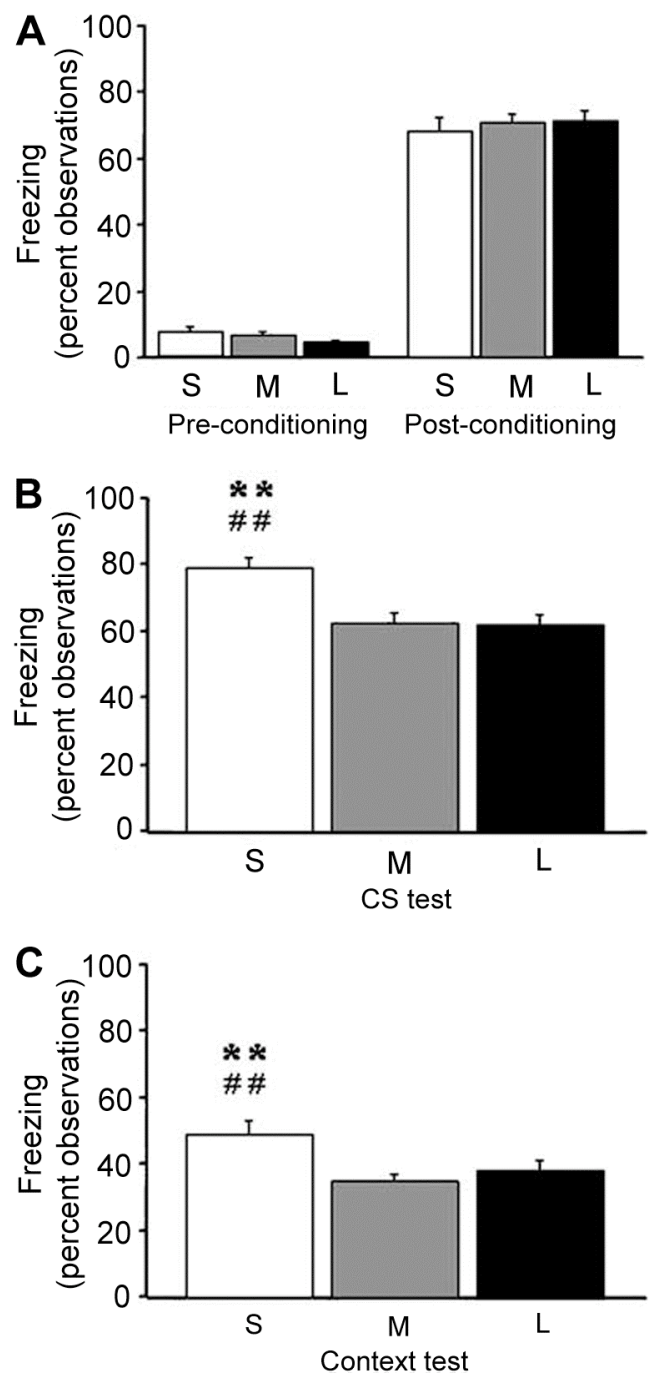

Figure 3-2 Pavlovian fear conditioning in mice differing in BLA volume

(A) There were no differences in freezing during the preconditioning and immediate postconditioning periods between mice with relativel small (S), medium (M), or large (L) BLA volume. The small BLA group froze more than medium or large BLA groups during exposure to the conditioned stimulus (CS) (B) and conditioned context (C) (n= $35-75$ per volume group). ${ }^{* *} p<0.01,{ }^{*} p<0.05$ vs L, ${ }^{\# \#} p<0.01$ vs. M. 
Table 3-1 Genetic correlations between BLA, striatum, hippocampus, cerebellum and behavior.

\begin{tabular}{|c|c|c|c|c|}
\hline Behavior & BLA & Striatum & Hippocampus & Cerebellum \\
\hline \multicolumn{5}{|l|}{ Pavlovian fear conditioning } \\
\hline Preconditioning freezing & -0.23 & -0.04 & -0.33 & 0.52 \\
\hline Postconditioning freezing & -0.03 & -0.05 & -0.17 & 0.20 \\
\hline Freezing to conditioned cue & $-0.50 *$ & -0.21 & -0.42 & -0.03 \\
\hline $\begin{array}{l}\text { Freezing to conditioned } \\
\text { context }\end{array}$ & -0.29 & 0.06 & -0.06 & 0.21 \\
\hline \multicolumn{5}{|l|}{ Forced swim test } \\
\hline Immobility & -0.13 & -0.30 & 0.02 & -0.25 \\
\hline \multicolumn{5}{|l|}{ Corticosterone } \\
\hline Baseline & -0.10 & $-0.42 *$ & -0.24 & - \\
\hline Poststress & -0.49 & 0.18 & 0.18 & 0.37 \\
\hline \multicolumn{5}{|l|}{ Novel open field } \\
\hline Distance traveled & $0.50 *$ & 0.04 & 0.30 & -0.41 \\
\hline Center time & 0.34 & 0.18 & 0.39 & -0.12 \\
\hline \multicolumn{5}{|l|}{ Elevated plus maze } \\
\hline Time in open arms & 0.04 & -0.47 & -0.31 & -0.12 \\
\hline Time in center square & $0.65^{*}$ & 0.43 & 0.16 & -0.05 \\
\hline Total arm entries & 0.38 & 0.21 & -0.02 & -0.07 \\
\hline Open arm entries & 0.02 & -0.35 & -0.06 & -0.23 \\
\hline Closed arm entries & 0.43 & 0.42 & 0.06 & 0.22 \\
\hline \multicolumn{5}{|l|}{ Dark-light exploration } \\
\hline Time in light & 0.37 & -0.12 & 0.27 & -0.24 \\
\hline Light-dark transitions & 0.34 & -0.20 & 0.11 & -0.24 \\
\hline
\end{tabular}

BLA, basolateral amygdala complex, ${ }^{*} p<0.05$ 

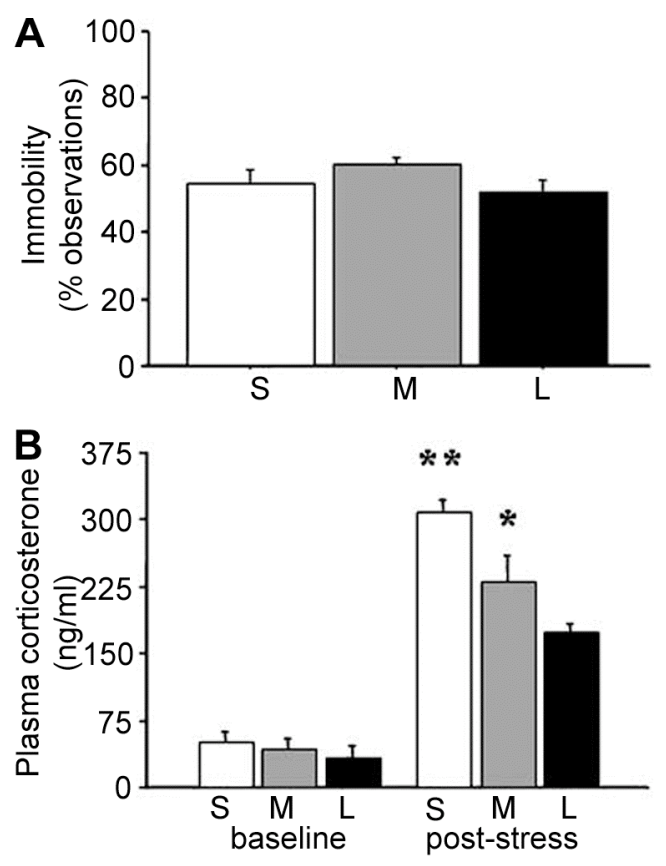

Figure 3-3 Depression-related behavior and glucocorticoid responses to stress in mice differing in BLA volume

(A) BLA volume was not associated with differences in percent immobility in the forced swim test ( $\mathrm{n}=25-49$ per volume group). (B) Mice with relatively small (S) BLA showed normal baseline corticosterone, but higher corticosterone responses to swim stress than medium $(\mathrm{M})$ or large $(\mathrm{L})$ BLA groups $(\mathrm{n}=5-\beta 11$ per volume group per stress condition). ${ }^{* *} p<0.01, \stackrel{*}{p}<0.05$ vs. L. 

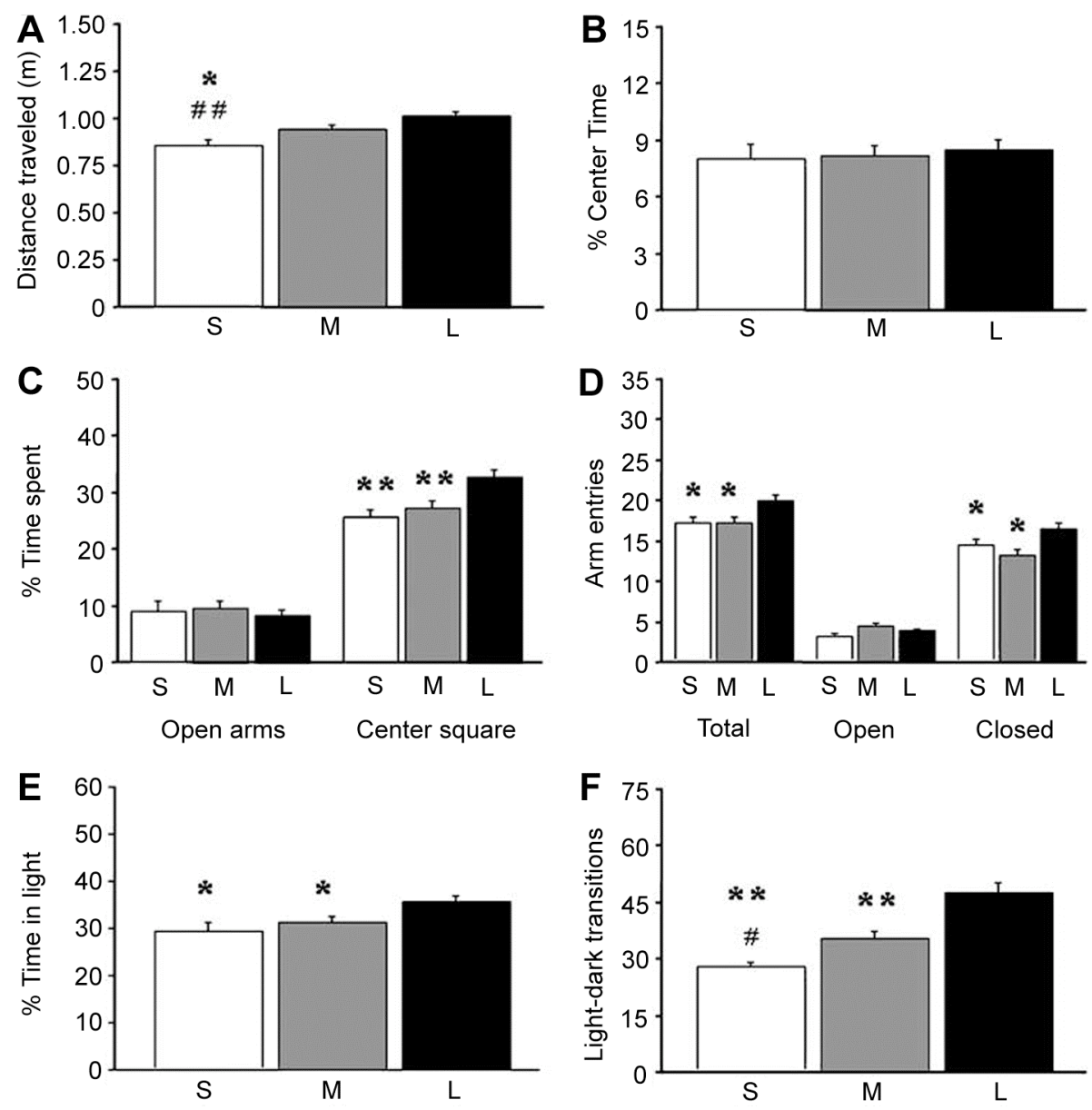

Figure 3-4 Exploratory- and anxiety-like behaviors in mice differing in BLA volume

Mice with relatively small (S) BLA traveled less distance than mice with medium (M) or large (L) BLA (A) while groups showed equivalent percent center time (B). Small and medium BLA groups spent less time in the center square but not open arms $(\mathbf{C})$ and made fewer total and closed but not open arm entries (D) in the elevated plus-maze than the large BLA group. Small and medium BLA groups spent less time in the light compartment (E) and made fewer light-dark transitions (F) in the light-dark exploration test than the large BLA group ( $\mathrm{n}=35-84$ per volume group) ${ }^{* *} p<0.01,{ }^{*} p<0.05$ vs. $\mathrm{L}$, $\# p<0.01, \stackrel{\# p}{p}<0.05$ vs. M. 
was a significant genetic correlation between distance traveled and volume of BLA, but not striatum, hippocampus or cerebellum (Table 3-1).

There was a significant effect of BLA group on total $\left(\mathrm{F}_{2,183}=4.24, p<0.05\right)$ and closed arm entries $\left(\mathrm{F}_{2,183}=6.93, p<0.01\right)$ and percent time in the center square $\left(\mathrm{F}_{2,183}=\right.$ $8.28, p<0.01)$, but not percent open arm time or open arm entries in the elevated plusmaze. The medium and small BLA groups spent less time in the center square (Figure 3-4C) and made fewer total and closed arm entries (Figure 3-4D) than the large BLA volume group. There was a significant genetic correlation between percent center time and volume of BLA, but not striatum, hippocampus, or cerebellum (Table 3-1).

There was a significant effect of BLA group on percent time in the light compartment $\left(\mathrm{F}_{2,175}=4.14, p<0.05\right)$ and light-dark transitions $\left(\mathrm{F}_{2,175}=13.16, p<0.01\right)$ in the light-dark exploration test. The medium and small BLA groups spent less time in the light compartment (Figure 3-4E) and made fewer light-dark transitions (Figure 3-4F) than the large BLA volume group, while the medium group made fewer transitions than the large group. Neither measure significantly correlated with volume of BLA, striatum, hippocampus, or cerebellum (Table 3-1).

\subsection{Discussion}

The major finding of the present study was that variation in volume of the BLA complex in a panel of BXD RI inbred strains was associated with differences in specific measures of mouse fear-, anxiety-, depression-, and stress-related phenotypes.

There is compelling evidence that the BLA is necessary for the formation and expression of associative fear memories, as typically evidenced by the effects of lesions on the type of Pavlovian fear conditioning task employed in our study (Davis \& Whalen, 2001; Fanselow \& Poulos, 2005; Phelps \& LeDoux, 2005). The present findings reinforce and extend this literature by showing that mice with naturally occurring variation in BLA volume exhibit significant differences in fear conditioning. BXD RI strains selected for BLA volumes that were relatively small showed significantly greater levels of conditioned freezing than strains with a larger BLA. There were no differences in levels of freezing immediately prior to or immediately after conditioning, demonstrating that differences between BLA groups in the recall/expression of fear memory were not due to differences in unconditioned freezing or in capacity to express short-term fear memory. Moreover, pain perception measured in the hot plate test did not differ between the groups, excluding variation in pain perception as a confounding influence.

Differences in fear expression between BLA volume groups were found both for conditioned fear to discrete (auditory cue) and compound (context) stimuli, which is consistent with the ability of BLA lesions (Goosens \& Maren, 2001) and gene knockoutinduced gross disruption of BLA (Lin et al., 2005) to impair both forms of associative fear memory. Interestingly however, there was a significant genetic correlation between 
BLA volume-varying BXD lines and cued, but not contextual, conditioned fear. A relatively greater correlation for cued conditioning is in agreement with evidence from lesion studies demonstrating the importance of the BLA for cued relative to contextual fear conditioning, the latter involving greater recruitment of additional structures, notably the hippocampus (Maren \& Quirk, 2004). These data also concur with gene-mapping studies in BXD RIs indicating that cued and contextual conditioning are under partly dissociable genetic control (Owen et al., 1997; Wehner et al., 1997). Finally, the fact that we were able to detect two correlating phenotypic traits (BLA volume and fear memory) across two laboratories (University of Tennessee, NIH) speaks both to the strength and replicability of the underlying genetic component (Wahlsten et al., 2006).

BLA volume-related variation in fear conditioning was dissociated from differences in anxiety-like behavior. The small BLA volume group showed less locomotor exploration in the novel open-field and elevated plus-maze than the larger BLA groups, but there were no differences in the principal measures of anxiety-like behavior (open-field center time, elevated plus-maze open arm exploration). In a third test of anxiety-like behavior, the light-dark exploration test, the small and medium BLA groups spent less time in the light compartment and made fewer light-dark intercompartment transitions than the large BLA group. Different mouse tests for anxiety likely measure different forms of behavior controlled by distinct genetic factors (Turri et al., 1999; Holmes et al., 2003; Kliethermes \& Crabbe, 2006) and it is possible that the profile of the small and medium BLA groups in the light-dark exploration test reflects a high anxiety-like phenotype that is not seen in the other tests. Taken together however, the differences between BLA volume groups on the anxiety-related assays (particularly the open-field and light-dark exploration tests) were marginal and are most parsimoniously interpreted as modest locomotor inhibition in response to novelty rather than a clear high anxiety-like phenotype in mice with smaller BLA volumes. Indeed, the absence of anxiety-related differences would be consistent with the lack of effects of BLA lesions or temporary inactivation on anxiety-like behaviors in rats tested under the standard task conditions used in the current study (Decker et al., 1995; Moller et al., 1997; Herry et al., 2007; Moreira et al., 2007).

To our knowledge, there has been little investigation of the role of BLA in rodent depression-related behaviors; with one study finding no effect of BLA lesions in the rat FST for depression-related behavior (Shimazoe et al., 1988). The present data found no link between variation in BLA volume and immobility in the mouse FST. By contrast, BLA groups differed markedly in their glucocorticoid responses to swim stress. Thus, while baseline levels of corticosterone were no different between the groups, corticosterone levels following swim stress were significantly greater in small BLA mice than in the larger BLA groups.

A pathway from the main output nucleus of the amygdala, the CeA, to the paraventricular nucleus of the hypothalamus (Swanson \& Petrovich, 1998) is thought to modulate hypothalamic-pituitary-adrenal (HPA) axis responses to stress (reviewed in Herman et al., 2005). Lesions encompassing the CeA can inhibit HPA-axis responses to at least some forms of stress (Beaulieu et al., 1986; Van de Kar et al., 1991), whereas 
amygdala stimulation can increase glucocorticoid release (Redgate \& Fahringer, 1973; Dunn \& Whitener, 1986). As such, the relatively greater HPA-axis response in small BLA mice could be another manifestation of abnormal amygdala function.

Corticosterone also exerts effects within the amygdala via glucocorticoid receptors (Aronsson et al., 1988). Of particular interest in this context, BLA has been identified as a critical site of action through which glucocorticoids modulate associative fear memory, (for example) glucocorticoid receptor agonists administered systemically or directly into the BLA enhance fear conditioning in rats (Zorawski \& Killcross, 2002; Hui et al., 2004; Roozendaal et al., 2006). This raises the possibility that increased glucocorticoid receptor activity in smaller BLA mice may be a factor driving enhanced fear conditioning, although this remains to be tested. A related issue is whether BLA variation is a consequence rather than cause of differences in stress reactivity. While we do not discount this possibility it seems unlikely given evidence that chronic stress increases rather than decreases spine density and dendritic arborization in rats and mice (Vyas et al., 2002; Mitra et al., 2005; Govindarajan et al., 2006). On the other hand, because stress causes dendritic shrinkage in rodent ventromedial prefrontal cortex (vmPFC) (Wellman, 2001; Izquierdo et al., 2006; Radley et al., 2006), it would be of interest to examine vmPFC and fear-related behaviors mediated by vmPFC such as fear extinction (Quirk \& Mueller, 2007) in mice with different BLA volumes.

BLA groups were selected for differences in BLA volume; and the fact that groups comprised multiple RI strains, each with a unique genotype, reduces the likelihood that differences in fear conditioning and stress reactivity were due to spurious idiosyncrasies (for example poor vision, hearing, nociception) of specific strains. However, this approach cannot exclude the potential contribution of other phenotypic traits that covary with BLA volume. We have recently shown that a common genetic locus may underlie the structures of multiple forebrain regions including amygdala, cerebellum, dorsal striatum, and portions of the hippocampus (Mozhui et al., 2007). Analysis of the volume of these three structures in our three BLA volume groups indicated volume differences in dorsal striatal but not hippocampal or cerebellar volume, raising the possibility that striatal volume variation contributed to behavioral differences between BLA groups, (for example via affects on motor function). Arguing against this possibility, however, there were no significant correlations between striatal volume and any behavioral measure (c.f. baseline corticosterone), while BLA volume significantly correlated with novel open-field locomotion. Moreover, BLA volume predicted corticosterone responses to stress, unlikely to be influenced by locomotion, while other measures predicted to be affected by locomotor differences, such as FST immobility, were unrelated to BLA volume. Nonetheless, although present data suggest a specific influence of amygdala function on fear conditioning and stress reactivity, we cannot rule out the possible contribution of differences in other brain regions or other, as yet undetermined, traits genetically correlated with BLA volume. In fact, given the highly integrated nature of the neural circuitry subserving stress, fear, and anxiety behaviors (Amat et al., 2005; Hariri \& Holmes, 2006; Quirk \& Mueller, 2007; Ressler \& Mayberg, 2007; Wellman et al., 2007), it is unlikely that the amygdala would act in isolation to mediate these complex functions. 
The finding that relatively smaller BLA is associated with increased fear and stress reactivity may at first appear counterintuitive given the effects of amygdala lesions, but this is in fact entirely consonant with recent findings from genetic studies in humans. For example, functional neuroimaging studies of two gene variants implicated in stressrelated disorders, the low-expressing forms of the serotonin transporter and monoamine oxidase A, demonstrate that these variants produce exaggerated amygdala reactivity during fear processing and that this is coupled with lesser amygdala volume (Pezawas et al., 2005; Meyer-Lindenberg et al., 2006). The volume differences underlying these fearrelated phenotypes were modest, as they were in our mouse sample. Thus, it appears that relatively modest variation in BLA volume is sufficient to determine fear- and stressrelated phenotypes.

Elucidating the molecular and physiological factors associated with volume differences is likely to be key to understanding this relationship between BLA volume and behavior. A pertinent observation in this regard is that the total number of neurons (and non-neuronal cells) in BLA was equivalent across BLA volume groups (Mozhui et al., 2007), indicating that rather than having a relative loss of neurons, the smaller BLA mice had a slightly denser packing of neurons (Mozhui et al., 2007). An important question for future studies will be how variation in this and other intrinsic properties of BLA, such as neuronal morphology and the ratio of excitatory over inhibitory cells, might contribute to the behavioral differences between BLA volume groups. Another interesting question is whether phenotypic differences between BLA groups are localized to specific subnuclei within the BLA complex. For example, akin to the small BLA phenotype observed in our study, lesions of the lateral nucleus prior to fear conditioning block acquisition (Goosens \& Maren, 2001; Nader et al., 2001; Koo et al., 2004), postconditioning lesions of the basal nucleus (corresponding to basolateral nucleus in Figure 3-1A) impair the expression, but not acquisition, of fear memory (AngladaFigueroa \& Quirk, 2005).

In summary, the present study has shown that variation in BLA volume is associated with differences in fear memory, glucocorticoid responses to stress, and locomotor inhibition in response to novelty. The present findings also provide parallels with clinical data, to the extent that PTSD patients also exhibit enhanced fear memory (American Psychiatric Association, 1994; Peri et al., 2000) and exaggerated glucocorticoid responses to stress (Yehuda, 2002; de Kloet et al., 2006), as well as amygdala hyperactivity and possibly smaller amygdala volume (Bremner et al., 1997; Wignall et al., 2004; Karl et al., 2006; Rauch et al., 2006; Shin et al., 2006), relative to controls. Thus, the present study not only provides a novel extension to the literature linking the amygdala with fear- and stress-related processing with possible implications for understanding amygdala dysfunction in PTSD, but also provides one of the first examples of how structural variation in a specific brain region can successfully predict differences in specific phenotypic traits in the rodent. 


\section{CHAPTER 4. GENETIC VARIATION IN STRESS SUSCEPTIBILITY IS ASSOCIATED WITH DIVERGENT CORTICOLIMBIC GENE EXPRESSION}

\subsection{Synopsis}

Genetic factors have significant influence in determining an individual's susceptibility to the adverse effects of stress. In this study, we used diverse inbred mouse strains as models to examine the genotype dependent effect of stress. Seven different inbred strains - 129/SvImJ, A/J, BALB/cJ, BALB/cByJ, C57BL/6J (B6), DBA2/J (D2), and $\mathrm{FVBN} / \mathrm{J}$ - were subjected to repeated restraint stress. Their relative vulnerability to stress was then assessed using different behavioral and physiological indices. We found significant strain differences in anxiety- and depression-like phenotypes, and differences in blood corticosterone level in response to stress. Of particular interest was the behavioral divergence between B6 and D2. D2 showed a much higher basal anxiety-like behavior than B6, and anxiety level was heightened by restraint stress. In contrast, B6 appeared more resilient to stress and did not show a significant difference in anxiety-like behavior before and after the restraint treatment. We further assayed global gene expression in the prefrontal cortex, amygdala, and hippocampus of B6 and D2 mice. Our analysis detected substantial expression difference between B6 and D2 in all three regions. We also identified a large set of genes that showed expression change in response to restraint stress, including several genes that have been associated with psychiatric illnesses in human (e.g., Homerl, $\mathrm{Nr} 4 a$ 2, Gal). In addition, several clock genes involved in regulating the circadian rhythm (Per1, Per2, Per3, Dbp) showed an upregulation following restraint, suggesting possible links between the molecular mediators of stress and the sleep cycle.

\subsection{Introduction}

Stress is a known major risk factor for neuropsychiatric diseases ranging from mood and anxiety disorders to schizophrenia and drug addiction. Susceptibility to stress varies considerably between individuals and is under the influence of genetic factors (Caspi \& Moffitt, 2006; Hariri \& Holmes, 2006; Yehuda \& LeDoux, 2007; Uher \& McGuffin, 2008). However, despite being the subject of enormous research efforts, the specific genes underlying vulnerability and resilience to stress have not yet been well established. This is in part due to the complexity that arises from multiple genetic, lifestyle and environmental factors in human populations.

Rodents provide important model systems for identifying genetic factors that influence vulnerability to stress (Ramos \& Mormede, 1998; Cryan \& Holmes, 2005). Of considerable value are inbred mouse strains. A panel of inbred strains can represent a restricted degree of environmental variance, within which each individual strain provides a stable genetic pool. Previous studies have shown that inbred strains differ in their responses to stress on various neural, neuroendocrine and behavioral measures. For example, acute exposure to stress produces heightened anxiety-like behavior and 
hypothalamic-pituitary-adrenal (HPA)-axis activation in some strains, such as BALB/cJ and D2, but less so or not at all in others, such as B6 (Belzung \& Griebel, 2001; Anisman \& Matheson, 2005; Jacobson \& Cryan, 2007; Millstein \& Holmes, 2007). Inbred mouse strains have also been shown to differ in their responses to repeated stress exposure (Tannenbaum \& Anisman, 2003; Anisman \& Matheson, 2005; Mineur et al., 2006), although there have been surprisingly few studies of this kind to date. This differential sensitivity to repeated stress is particularly pertinent to attempts at modeling human neuropsychiatric diseases because risk for these disorders is often associated with history of repeated trauma (Caspi et al., 2002; Berton \& Nestler, 2006; Yehuda \& LeDoux, 2007; Holmes \& Wellman, 2009).

In the present study, we surveyed anxiety-, depression- and HPA-axis responses following repeated stress in genetically diverse inbred mice. This multiple strain comparison was done using a panel of seven inbred mice. The aim was to identify strains that differ in anxiety-related traits and sensitivity to repeated stress. To this end, we employed a repeated restraint stress protocol that has been shown to cause significant alterations in the morphology of principal neurons in three key corticolimbic regions known to mediate the effects of stress: medial prefrontal cortex (mPFC), amygdala and hippocampus (e.g., Vyas et al., 2002; Vyas et al., 2003; Govindarajan et al., 2006; Bennur et al., 2007; Shansky et al., 2009). Based on the results obtained, our second major aim was to examine gene expression in $\mathrm{mPFC}$, amygdala and hippocampus in two strains (B6 and D2) that differed in anxiety-like behavior before and after restraint.

\subsection{Materials and Methods}

\subsubsection{Subjects}

Subjects were male mice of the 129/SvImJ (129S1), A/J, BALB/cJ, BALB/cByJ, C57BL/6J (B6), DBA2/J (D2), and FVBN/J inbred strains. These strains were selected on the basis of 1) their frequent use in behavioral neuroscience and as genetic backgrounds for mouse mutant lines, 2) inclusion as "group A" priority strains in the Mouse Phenome Project, an international effort to provide the biomedical research community with phenotypic data on the most commonly used mouse strains (http://www.jax.org/phenome), and 3) prior studies demonstrating differential trait anxiety- and depression-related and stress-sensitivity phenotypes (Anisman \& Matheson, 2005; Millstein \& Holmes, 2007). Mice were obtained from The Jackson Laboratory (Bar Harbor, ME) to reduce a potential source (i.e., supplier) of genetic and behavioral variation. Mice were aged 8-9 wks at the start of the study, housed 2/cage (by strain and stress condition), with cages placed side-by-side in a temperature- $\left(72 \pm 5^{\circ} \mathrm{C}\right)$ and humidity- $(45 \pm 15 \%)$ controlled vivarium under a 12 hour light/dark cycle (lights on 0600 hour). Testing was conducted in a manner counterbalanced for strain and stress condition. The number of mice used is given in the figure legends. All experimental procedures were approved by the National Institute on Alcohol Abuse and Alcoholism Animal Care and Use Committee and followed the National Institute of Health guidelines 
outlined in 'Using Animals in Intramural Research' and the local Animal Care and Use Committees.

\subsubsection{Repeated Restraint Stress}

Previous studies have shown that 10 days of immobilization in 'immobilization bags' produces significant changes in neuronal morphology in the $\mathrm{mPFC}$, basolateral amygdala and CA3 region of the hippocampus in rats and mice (Vyas et al., 2003; Govindarajan et al., 2006; Shansky et al., 2009). We adopted a modified version of this protocol in which mice were placed in ventilated $50 \mathrm{~mL}$ Falcon tubes for 2 hour/day $(1000-1200 \mathrm{hr})$ for 10 consecutive days. To be consistent with earlier studies using this stressor in rats and mice (Vyas et al., 2002; Govindarajan et al., 2006; Shansky et al., 2009), non-stressed controls remained in the home cage. We reasoned that restraint in tubes would be a less severe stressor than restraint in immobilization bags and would therefore allow us to better detect differential sensitivity to stress across strains than a severe stressor that might cause profound changes across all strains.

\subsubsection{Effect of Repeated Restraint on Body Weight}

To obtain a systemic measure of the effects of restraint, we measured reductions in body weight (Willner et al., 1996; Pothion et al., 2004; Krishnan et al., 2007; Shansky et al., 2009). Changes in body weight over the 10-day restraint period were compared between restrained and non-restrained groups. Statistical significance for this and all other analyses was set at $p \leq 0.05$.

\subsubsection{Effect of Repeated Restraint on Anxiety-Related Behavior}

Twenty-four hours after the final restraint, mice were tested for anxiety-like behavior using the dark-light emergence test, modified from a procedure previously described (Holmes et al., 2003). We employed this task rather than more commonly used tests for anxiety-like behavior, such as the elevated plus-maze (Cryan \& Holmes, 2005), because under baseline conditions in our laboratory, B6 mice typically display lesser anxiety-like behavior in the emergence test $(\sim 25 \%$ time out of shelter (e.g., this study) than in the elevated plus-maze ( $\sim 10 \%$ open arm time (e.g., Norcross et al., 2008). Thus, we predicted that emergence test would facilitate the detection of increases in anxietylike behavior caused by stress by avoiding a 'floor effect.'

The mouse was placed in an opaque black Plexiglas shelter $(39 \times 13 \times 16 \mathrm{~cm})$ with a $13 \times 8 \mathrm{~cm}$ aperture at floor level that opened onto a large white Plexiglas square arena $(39 \times 39 \times 35 \mathrm{~cm})$ illuminated to $\sim 90$ lux. Latency to first exit the shelter, the number of shelter exits (defined as all 4 paws out of the shelter) and time spent out of the shelter in the open field over a 15 min session was recorded by an observer using Hindsight (Hindsight, Services, Wokingham, UK). To dissociate the anxiety-related and 
general exploratory phases of the test session, data were separately analyzed during the first $5 \mathrm{~min}$ and last $5 \mathrm{~min}$, respectively. The effects of strain and stress were analyzed using 2-factor analysis of variance (ANOVA) followed by Newman Keuls post hoc tests.

Given the high number of strains tested and the resultant reduction of power in our analysis, we conducted planned post hoc comparisons of strain and/or stress effects in the presence of significant ANOVA main effects regardless of whether a significant strain $\mathrm{x}$ stress interaction effect was also found.

\subsubsection{Effect of Repeated Restraint on Depression-Related Behavior}

Twenty-four hours after the emergence test, mice were assessed for depressionrelated behavior in the forced swim test (Porsolt et al., 1977; Cryan \& Holmes, 2005). Mice were gently lowered into a transparent Plexiglas cylinder $(20 \mathrm{~cm}$ diameter $)$ filled halfway with water $\left(24 \pm 1^{\circ} \mathrm{C}\right)$ for a 6 min session, as previously described (BoyceRustay \& Holmes, 2006). The presence/absence of immobility (cessation of limb movements except minor involuntary movements of the hind limbs) was manually observed using an instantaneous sampling technique every $5 \mathrm{sec}$ during min 2-6 and expressed as a percentage of total observations. The effects of strain and stress were analyzed using 2-factor ANOVA followed by Newman Keuls post hoc tests.

\subsubsection{Effect of Repeated Restraint on Swim Stress Activation of HPA-Axis}

Mice exposed to repeated social defeat stress exhibit an augmented HPA-axis response to swim stress (Krishnan et al., 2007). We tested whether repeated restraint stress similarly sensitized the HPA-axis response to swim stress. Restraint stressed mice and non-restrained controls were returned to the home cage after the forced swim stress session described above. Thirty min later, mice were sacrificed via rapid cervical dislocation and decapitation to collect trunk blood. An additional set of experimentallynaïve mice were sacrificed at the same time to provide non-swim-stressed baseline corticosterone data. Blood samples were centrifuged at 13,000 rpm for $30 \mathrm{sec}$. Serum was extracted and assayed for total corticosterone (bound and free) using the Coat-a-Count RIA TKRC1 kit (limit of detection: 5.7 ng/ml; Diagnostic Products Corp, Los Angeles) as previously described (Boyce-Rustay et al., 2007). The effects of strain and stress were analyzed using 2-factor ANOVA followed by Newman Keuls post hoc tests.

\subsubsection{Sample Preparation for Expression Analysis}

We next performed genome-wide gene expression analysis of B6 and D2 mice in order to compare changes in gene expression following restraint stress in the two strai. Mice were exposed to repeated restraint (as above), and then sacrificed via cervical dislocation and rapid decapitation 4 hour after the final restraint stress. A set of naïve D2 and B6 mice was also sacrificed at the same time. Brains were quickly removed and 
stored in RNAlater (Ambion, Austin, TX). Three corticolimbic brain regions mediating stress- emotion-related behaviors, the medial prefrontal cortex (mPFC), amygdala (principally the basolateral nucleus) and hippocampus, were microdissected within 48 hour of brain collection. The brain was placed in a coronal matrix and sectioned 1.4-2.4 $\mathrm{mm}$ from Bregma to obtain the mPFC (tissue medial to the forceps minor, mainly comprising the infralimbic and prelimbic cortices), and 1.0-2.0 $\mathrm{mm}$ from Bregma to obtain the amygdala and hippocampus. The amygdala was visualized under dark-field microscope and dissected using the external and internal capsules as a guide to obtain the basolateral nucleus (although we cannot exclude some inclusion of tissue from the central nucleus and striatum). The whole hippocampus was then dissected.

Tissue was immediately frozen and stored at $-80^{\circ} \mathrm{C}$. Samples from each mouse were stored and analyzed separately (i.e., no pooling). Total RNA was isolated using RNAqueous Micro kit (Ambion, Austin, TX). RNA purity and concentration was evaluated with a spectrophotometer using a 260/280 $\mathrm{nm}$ absorbance ratio, and RNA quality was checked using Agilent Bioanalyzer 2100 (Agilent Technologies, Santa Clara, CA). Samples were processed according to the manufacturer's instructions and hybridized onto a total of 36 Illumina Mouse-6.1 arrays (Illumina, Inc, San Diego, CA). Samples that differed by strain, region, and treatment were interleaved on arrays on the same slide (6 arrays per slide) to avoid batch confounds. Raw microarray data were normalized using the rank invariant and background subtraction protocols provided by Illumina as part of its BeadStation software suite. We log-transformed the expression values and stabilized the variance of each array. The Illumina Mouse-6.1 array consists of $>46,000$ probes. We extensively re-annotated these probes and the improved annotations were incorporated into the data analysis for the current study.

\subsubsection{Microarray Data Analysis}

Probes were initially filtered by expression: i.e., expression signals below background in half or more of the samples were excluded resulting in 25,908 probes for analyses. This set of expressed genes was analyzed by hierarchical clustering, using the TM4 MeV software (Saeed et al., 2003), to sort the samples based on global expression patterns. Baseline strain effects were defined as gene expression values in non-restrained B6 mice that showed both a fold-difference $\geq 1.3$ and a significant difference as determined by Student's t-test relative to values in non-restrained D2 mice. Restraint effects were defined as gene expression values in restrained vs. non-restrained mice of the same strain that showed both a fold-difference $\geq 1.3$ and a significant difference as determined by Student's t-test. 


\subsection{Results}

\subsubsection{Effect of Repeated Restraint on Body Weight}

We measured loss of body weight as a systemic measure of the stress caused by repeated restraint. ANOVA revealed a significant strain $\mathrm{x}$ restraint interaction for change in body weight over the 10 -day stress period $\left(\mathrm{F}_{6,84}=3.57, p<0.01\right)$. Post hoc tests showed that all strains except $129 \mathrm{~S} 1$ and D2 showed a significant difference in body weight change between the restrained and non-restrained groups (Figure 4-1). The lack of a statistically significant difference in D2 was likely due to high variability in the restrained D2 group in this experiment.

\subsubsection{Effect of Repeated Restraint on Anxiety-Related Behavior}

We measured behavior during the first 5 minutes of the dark-light emergence test as an assay for the effects of repeated restraint and strain differences in anxiety-like behavior. ANOVA found a significant stress $\mathrm{x}$ strain interaction $\left(\mathrm{F}_{6,98}=2.71, p<0.05\right)$ for the number of shelter exits. Post hoc analysis showed that all strains except FVBN/J made significantly fewer shelter exits than the reference B6 strain, while restrained D2 and FVBN/J mice made significantly fewer exits than their non-restrained D2 and

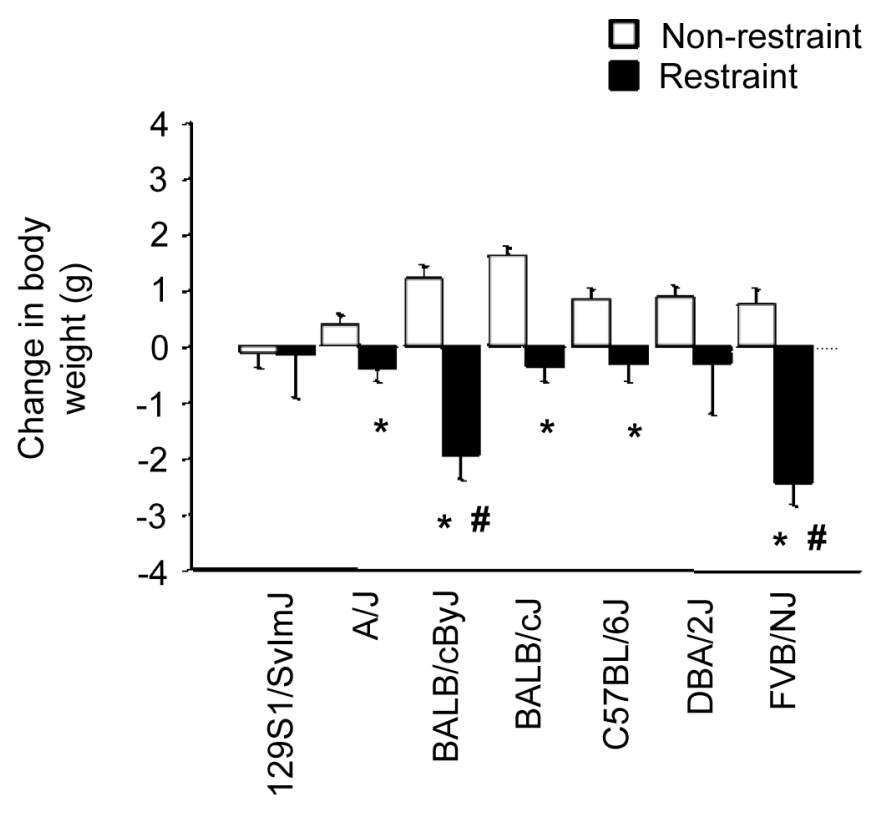

Figure 4-1 Differences in repeated restraint-induced changes in body weight Restraint stress reduced body weight in a strain-dependent manner, with significantly greater net reductions in $\mathrm{BALB} / \mathrm{cByJ}$ and $\mathrm{FVBN} / \mathrm{J}$ than the reference $\mathrm{C} 57 \mathrm{BL} / 6 \mathrm{~J}$ strain $\left(\mathrm{N}=5-9\right.$ per strain and condition). ${ }^{*} p<0.05$ vs. non-restraint mice of the same strain, $\# p$ $<0.05$ vs. C57BL/6J. Data are Means \pm SEM. 
FVBN/J counterparts (Figure 4-2A). There was also significant effect of strain $\left(\mathrm{F}_{6,98}\right.$ $=29.64, p<0.01$ ), but no main effect of stress and or strain $\mathrm{x}$ stress interaction, for percent time spent out of the shelter during the first $5 \mathrm{~min}$. Post hoc comparison between restraint and non restraint groups found that only restrained D2 spent significantly lesser time out of the shelter than non-restrained counterpart. Under baseline conditions, 129S1, $\mathrm{A} / \mathrm{J}, \mathrm{BALB} / \mathrm{cByJ}$, and BALB/cJ spent significantly less time, and FVB/NJ spent more time, out of the shelter than B6 (Figure 4-2B). Lastly, there was a significant effect of strain $\left(\mathrm{F}_{6,98}=18.45, p<0.01\right)$ but not stress, and no strain $\mathrm{x}$ stress interaction, for latency to first exit the shelter. At baseline, 129S1, A/J, BALB/cByJ, and BALB/cJ were significantly slower to exit the shelter than B6 (Figure 4-2C). Latency did not differ between restrained and non-restrained mice of any strain.

We reasoned that the final 5 minutes of the 15 minutes test session would be less sensitive than the first 5 minutes to true-positive strain- or restraint-related effects on anxiety-like behavior. During this period, there was a significant effect of strain for shelter exits $\left(\mathrm{F}_{6,98}=36.15, p<0.01\right)$ and for percent time out of the shelter $\left(\mathrm{F}_{6,98}=47.02\right.$, $p<0.01$ ), but no significant main effect of stress and no strain $\mathrm{x}$ stress interaction for either measure. Post hoc comparisons showed that all strains except D2 and FVBN/J made significantly fewer less shelter exits and spent less time out of the shelter, as compared to B6. Repeated restraint had no effect on either measure in any strain during this period.

\subsubsection{Effect of Repeated Restraint on Depression-Related Behavior}

We next examined depression-related behavior using the forced swim test. There was a significant effect of strain $\left(\mathrm{F}_{6,98}=58.24, p<0.01\right)$ and stress $\left(\mathrm{F}_{1,98}=5.61, p<0.05\right)$ but no strain $\mathrm{x}$ stress interaction for percent immobility. Post hoc analysis showed that D2 and FVB/NJ showed significantly less immobility than the B6 reference strain, and that restrained BALB/cByJ showed significantly less immobility than non-restrained BALB/cByJ counterparts (Figure 4-3).

\subsubsection{Effect of Repeated Restraint on the HPA-Axis}

Serum corticosterone levels were measured 30 minutes after forced swim stress. There was a significant stress $\mathrm{x}$ strain interaction $\left(\mathrm{F}_{6,152}=4.82, p<0.01\right)$, as well as main effects of stress $\left(\mathrm{F}_{2,12}=228.74, p<0.01\right)$ and strain $\left(\mathrm{F}_{6,12}=7.00, p<0.01\right)$, for corticosterone levels. Post hoc analysis showed that swim stress significantly increased corticosterone in all strains as compared to non-swim baseline, regardless of prior repeated restraint stress (Figure 4-4). In addition, swim stress-induced corticosterone levels were significantly higher in BALB/cJ, B6 and D2 following repeated restraint relative to their non-restrained counterparts. Conversely, repeatedly restrained 129S1 showed a significantly lesser corticosterone response to swim stress than non-restrained 129S1. 

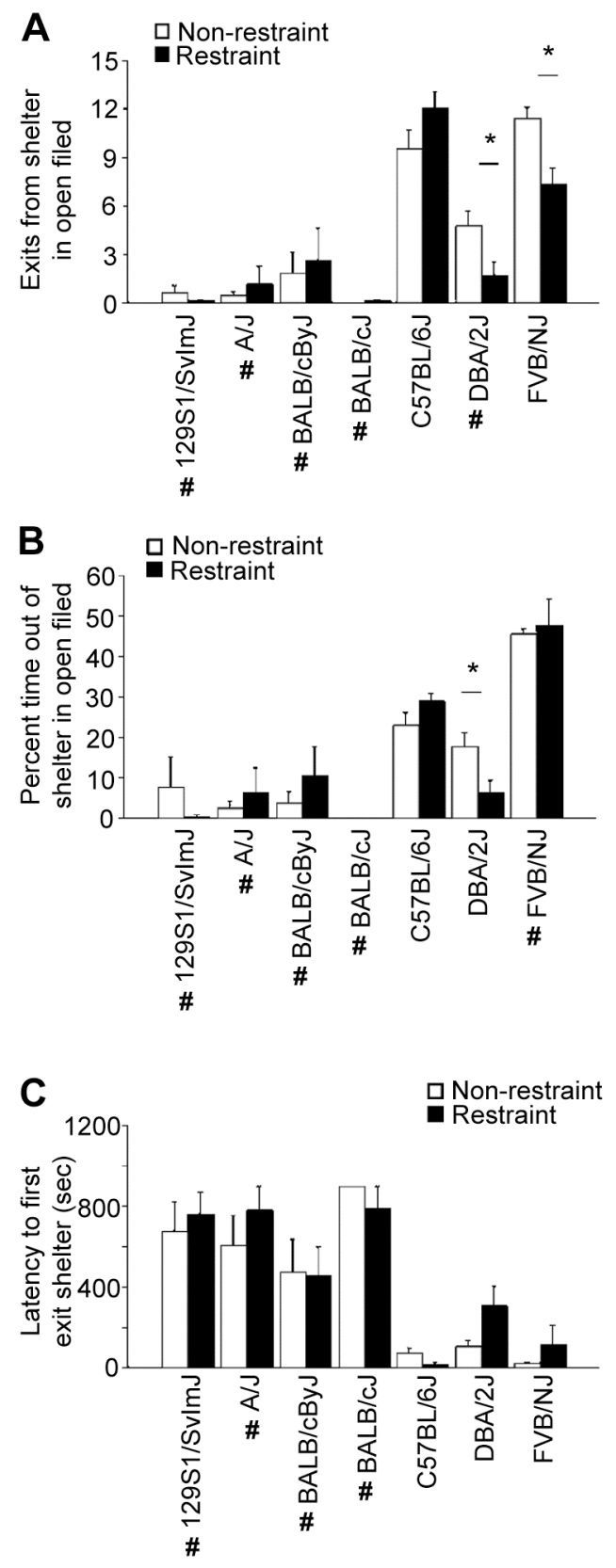

Figure 4-2 Differences in anxiety-like behavior in emergence test

(A) All strains except FVBN/J made significantly fewer shelter exits than the reference C57BL/6J strain, irrespective of stress. DBA/2J and FVBN/J mice, but no other strain, showed significantly fewer shelter exits following repeated restraint, as compared to nonrestrained mice. (B) DBA/2J mice, but no other strain, spent significantly less time out of the shelter following repeated restraint, as compared to non-restrained DBA/2J mice. (C) There was no difference between restraint and non-restraint groups in latency to first exit shelter. 129S1, A/J, BALB/cByJ, and BALB/cJ were significantly slower to exit the shelter than $\mathrm{B} 6 . \mathrm{N}=7-9$ per strain and condition. ${ }^{*} p<0.05$ vs. non-stressed controls of same strain; $\# p<0.05$ vs. C57BL/6J. Data are Means \pm SEM. 


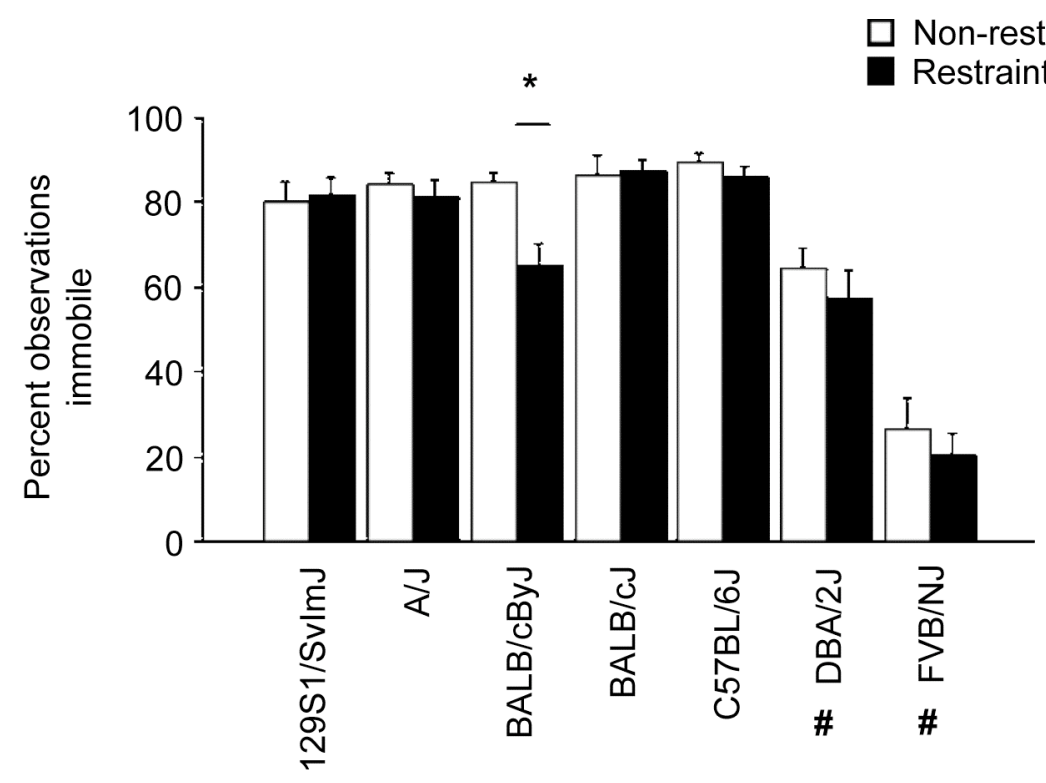

Figure 4-3 Differences in depression-related behavior

$\mathrm{DBA} / 2 \mathrm{~J}$ and FVBN/J mice were significantly less immobile than the reference C57BL/6J strain, regardless of stress. BALB/cByJ mice, but no other strain, showed significantly less immobility as compared to non-restrained BALB/cByJ mice. $\mathrm{N}=7-9$ per strain and condition. ${ }^{*} p<0.05 ; \# p<0.05$ vs. C57BL/6J. 


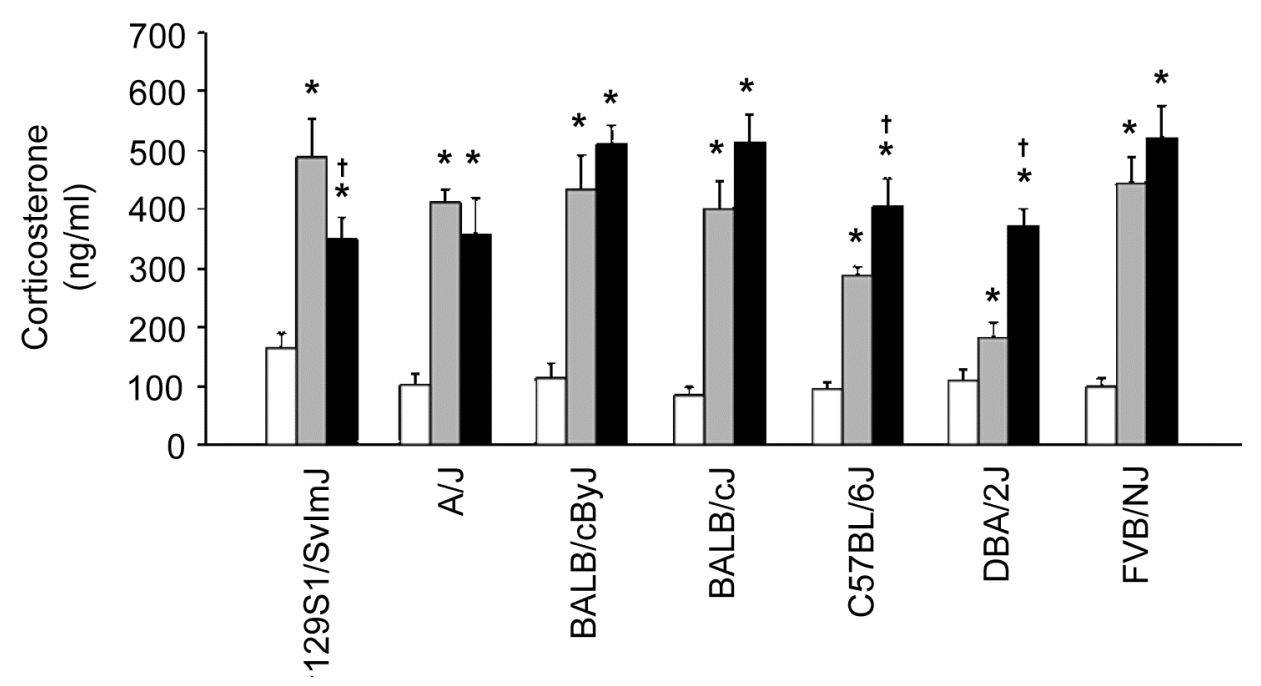

Figure 4-4 Differences in corticosterone responses to swim stress

Exposure to swim stress significantly increased serum corticosterone levels, relative to non-swim/non-restraint baseline, in all strains regardless of repeated restraint stress history. Swim stress-induced corticosterone levels were significantly lower in 129S1 and significantly higher in BALB/cJ, C57BL/6J and DBA/2J mice following repeated restraint, as compared to non-restrained counterparts. $\mathrm{N}=5-15$ per strain and condition. $* p<0.05$ vs. non-restrained of same strain; $\uparrow p<0.05$ vs. non-restrained and swim stressed of same strain. 


\subsubsection{Corticolimbic Gene Expression in B6 and D2}

Our behavioral analyses showed that B6 and D2 exhibited differences in anxietylike behavior, consistent with previous studies (Millstein \& Holmes, 2007). These differences further diverged following exposure to repeated restraint. On the basis of these observations, we conducted a genome-wide analysis of baseline gene expression differences between B6 and D2 in the amygdala, mPFC and hippocampus, as well as gene expression changes in each strain following repeated restraint.

Hierarchical cluster of all probes with gene expression signal above background showed that gene expression data segregated as a function of brain region, with relatively greater similarity in the amygdala and $\mathrm{mPFC}$ than the hippocampus (Figure 4-5). Within each brain region, samples segregated by strain and, to a lesser extent, restraint, demonstrating a stronger effect of strain than restraint on gene expression.

Basal gene expression differed markedly between B6 and D2 in all 3 regions, albeit with a greater number of differences in the hippocampus (2540 probes representing 2154 known genes) followed by the amygdala (1786 probes/1524 known genes) and then the mPFC (1712/1491 known genes) (Figure 4-6A). There was also considerable overlap across regions in the genes differentially expressed by the 2 strains, with 560 probes detecting differences common to all 3 regions.

We then assessed the extent of overlap in restraint-induced expression changes in the three brain regions. We considered all probes that detected expression changes following repeated restraint and compared them across tissues (Figure 4-6B). In contrast to the effect of strain, only a small subset of expression changes associated with restraint overlapped across 2 brain regions ( 36 between amygdala and hippocampus, 22 between amygdala and $\mathrm{mPFC}, 13$ between hippocampus and $\mathrm{mPFC}$ ) and only 5 probes detected expression changes in all 3 regions. Of these 5, 3 target known genes: Period 2 (Per2), D

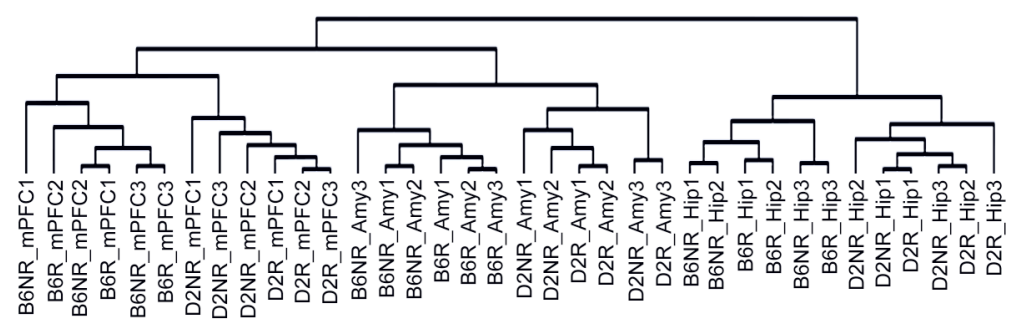

\section{Figure 4-5 Hierarchical cluster analysis of expression data}

Hierarchical clustering was done using 25,908 probes that detected expression signals above background in at least 18 out of 36 samples. The cluster analysis was able to group the samples by brain region and strain. Based on global gene expression patterns, the samples segregated into 3 main region-specific branches - medial prefrontal cortex (mPFC), amygdala (Amy), and hippocampus (Hip). These further bifurcated into C57BL/6J (B6) and DBA/2J (D2) branches. Samples from the same treatment conditions, restraint-stressed (R) or non-restraint (NR), also tended to group together. 


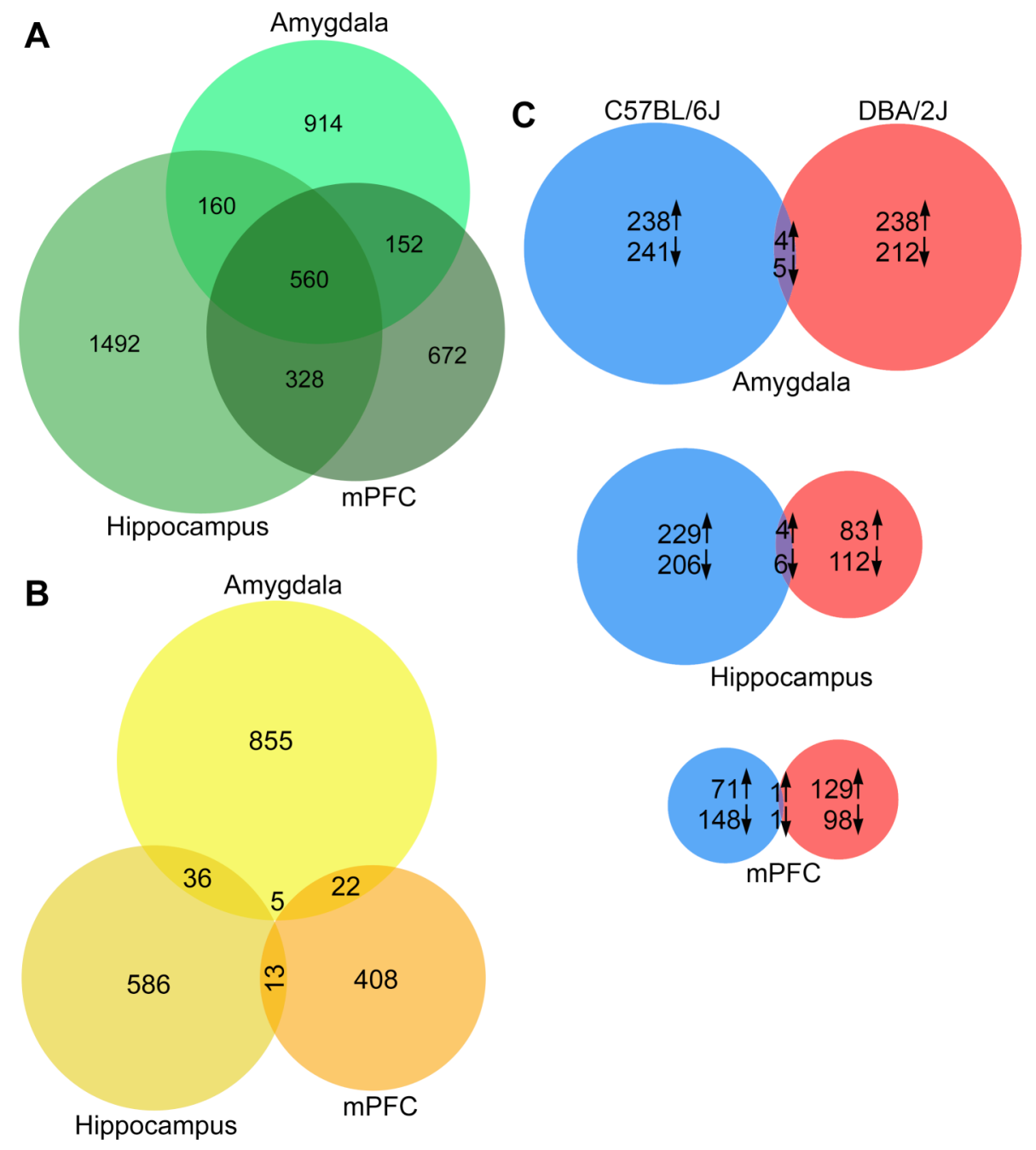

Figure 4-6 Differences in corticolimbic gene expression

(A) Basal gene expression differed markedly between C57BL/6J and DBA/2J. Relatively more strain difference in expression traits was evident in the hippocampus than the amygdala and mPFC. There was significant overlap in C57BL/6J and DBA/2J differentially expressed genes across the 3 region in the genes. (B) Overall, in both $\mathrm{C} 57 \mathrm{BL} / 2 \mathrm{~J}$ and DBA/2J, repeated restraint was associated with significant more gene expression changes in the amygdala, with significant but relatively fewer, changes in the hippocampus and medial prefrontal cortex (mPFC). A limited subset of expression changes overlapped across any 2 brain regions, and only 5 changes overlapped across all 3 regions. (C) C57BL/6J and DBA/2J mice showed approximately equal number of restraint-stress induced expression changes following repeated restraint in the amygdala and $\mathrm{mPFC}$, while C57BL/6J showed more changes in the hippocampus than DBA/2J. Gene expression changes associated with restraint were highly divergent between C57BL/6J and DBA/2J, with very few genes showing alterations in both these strains in any region. $\mathrm{N}=3$ per strain per condition. 
site of albumin promoter binding protein ( $D b p)$, and Claudin 5 (Cldn5). Notably, both Per 2 and $D b p$ are circadian rhythm regulator genes and have been associated with bipolar disorder (Shi et al., 2008). Cldn5 codes for a component of tight junctions and this gene has been implicated in susceptibility to schizophrenia (Sun et al., 2004). Both Per2 and $D b p$ are consistently upregulated by stress and $C l d n 5$ is downregulated by stress in all three regions (Table 4-1).

We then examined gene expression changes following restraint in B6 and D2. This revealed significant divergence between the two strains (Figure 4-6C). In both strains the amygdala showed the greatest number of restraint-induced gene expression changes (488/447 known genes in B6, and 439 probes/408 known genes in D2) but only 9 probes were common to both strains. In the hippocampus, B6 showed noticeably more expression changes after restraint than D2 (445 probes/401 known genes in B6, 205 probes/187 known genes in D2), and only 10 of these were common to both strains. For both the amygdala and hippocampus in B6 and D2, there were an approximately equal number of upregulation and downregulation following repeated restraint. In the $\mathrm{mPFC}$, B6 and D2 showed similar number of expression changes (221 probes/202 known genes in B6, 229 probes/211 known genes in D2); only 2 of these changes were in both the strains. In this region, there were more downregulation than upregulation for B6, but more upregulation than downregulation for D2.

\subsubsection{RT-PCR Analysis}

We used RT-PCR to confirm 16 gene expression differences associated with strain and/or restraint. Eight of these genes were selected on the basis of prior evidence linking the gene to stress-related behaviors, as in the case of Gal (Karlsson \& Holmes, 2006), Atpla2 (Ikeda et al., 2003), Bdnf (Nestler et al., 2002), Nr4a2 (Rojas et al., 2007), Chrna4 (Labarca et al., 2001), Comt (Zubieta et al., 2003; Papaleo et al., 2008) Drdla (Hains \& Arnsten, 2008), and Rgs2 (Yalcin et al., 2004). Four genes (Grikl, Grin1, Grial, and Homer I) are key components of glutamate-mediated neurotransmission and neural plasticity (Malenka \& Bear, 2004; Szumlinski et al., 2004; Szumlinski et al., 2005). A third set are circadian genes (Per 1, Per2, Per 3 , and Dbp) are increasingly implicated in neuropsychiatric diseases, notably mania (McClung, 2007).

Table 4-1 Restraint-stress induced upregulation of clock genes

\begin{tabular}{|c|c|c|c|c|c|c|}
\hline \multirow[b]{2}{*}{ Gene } & \multicolumn{2}{|c|}{ mPFC } & \multicolumn{2}{|c|}{ BLA } & \multicolumn{2}{|c|}{ Hippocampus } \\
\hline & B6 & D2 & B6 & D2 & B6 & D2 \\
\hline Per2 & $\uparrow 2.2^{* *}$ & $\uparrow 1.9^{*}$ & $\uparrow 1.8^{* *}$ & $\uparrow 1.5^{*}$ & ns & $\uparrow 1.6^{* *}$ \\
\hline$D b p$ & $\uparrow 2.0^{* * *}$ & $\uparrow 1.8^{*}$ & $\uparrow 1.8^{* *}$ & ns & $\uparrow 2.5^{* * *}$ & $\uparrow 1.8^{* *}$ \\
\hline Cldn5 & $\downarrow 1.8^{* *}$ & ns & $\downarrow 1.5^{* *}$ & ns & $\downarrow 1.7^{* *}$ & $\downarrow 2.2^{* *}$ \\
\hline
\end{tabular}

$$
{ }^{*} p<0.05, * * p<0.01
$$


In the amygdala, we found that basal expression of Grin1 was lower in B6 relative to D2 (Table 4-2). In addition, basal hippocampal expression of Grial was lower, and $B d n f$ higher, in $\mathrm{B} 6$ relative to D2. As compared to D2, microarray analysis found that B6 hippocampal expression of Atpla2 was lower, while RT-PCR analysis found that opposite pattern. As the microarray probes and RT-PCR primers do not perfectly target the same sequence, this inconsistency may be due to spliced variants (Korostynski et al., 2006). Finally, basal mPFC expression of Comt was higher, and expression of Chrna4 and Rgs2 lower, in B6 relative to D2.

Regarding expression differences between restrained and non-restrained B6, we found Grikl and Gal were downregulated, while Grinl was upregulated in the amygdala following restraint (Table 4-2). In the hippocampus, expression of $\mathrm{Nr} 2 \mathrm{a} 2$ was downregulated in B6 following restraint. Chrna4, Drdla and Per2 were all upregulated in the $\mathrm{mPFC}$ after restraint in B6. Atpla2 was upregulated according to the microarray analysis but downregulated according to RT-PCR, again suggesting the possibility of spliced variants.

We confirmed 4 expression differences between restrained and non-restrained D2 mice. In the amygdala, the expression of Perl was upregulated in restrained relative to non-restrained (Table 4-1) while in the hippocampus, Dbp was upregulated and Homerl was downregulated following restraint. Lastly, in the mPFC of D2, Per2 was upregulated in after restraint.

\subsubsection{Candidate Genes in BLA Volume QTL}

We previously identified a significant QTL for BLA volume on mid-chromosome 8, Vol8a (Mozhui et al., 2007). Using the BLA expression data, we searched for genes located in Vol8a that show significant basal expression difference between B6 and D2. Among the candidates that were previously identified in Mozhui et al. (2007), only Neurocan (Ncan or Cspg3) showed significant differential expression between B6 and D2 with 2.4-fold higher expression in B6 BLA than in D2 BLA $(p<0.001)$. Two other genes with substantial expression difference are sterol-C4-methyl oxidase-like ( $\mathrm{Sc} 4 \mathrm{~mol})$ and occluding/ELL domain containing 1 (Ocel1). While Sc4mol showed 4.3-fold higher expression in B6 than in D2 $(p<0.0001)$, Ocell showed 4-fold higher expression in D2 than in B6 $(p=0.0001)$. These genes that have strong strain difference in expression in the BLA are high priority candidates for differences in BLA volume.

\subsection{Discussion}

In this study, we analyzed anxiety-like behavior prior to, and following repeated restraint in seven inbred strains of mice. The major finding of the current study was that different strains of mice exhibit varying levels of vulnerability to restraint stress, and such differences may be associated with divergent corticolimbic expression of genes 
Table 4-2 Fold-difference scores for microarray and quantitative PCR

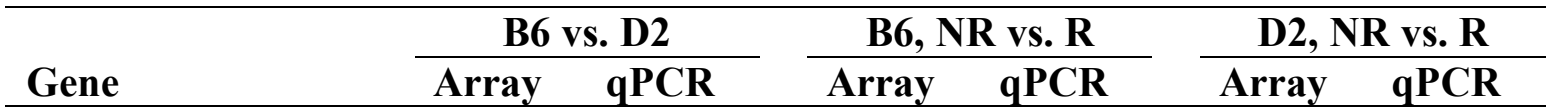

\section{Amygdala}

Grik 1 (glutamate

$\begin{array}{llll}- & - & \downarrow 2.2^{* *} & \downarrow 1.3^{*} \\ & & & \\ \downarrow 1.7^{*} & \downarrow 2.2^{*} & \uparrow 1.9^{* *} & \uparrow 2.2^{*}\end{array}$

receptor, ionotropic,

kainate 1)

Grinl (glutamate

receptor, ionotropic,

NMDA1)

Gal (galanin)

Perl (period

$\uparrow 4.9^{* *} \quad \mathrm{~ns}$

homolog 1)

\section{Prefrontal cortex}

Chrna4 (cholinergic

receptor, nicotinic, alpha polypeptide 4) Comt (catechol-Omethyltransferase) Drdla (dopamine receptor D1A)

Per2 (period

$$
\downarrow 1.7^{* *} \quad \downarrow 1.6^{*}
$$

$\uparrow 1.3 * *$

homolog 2)

$\operatorname{Rgs} 2$ (regulator of G-

$\uparrow 1.6^{* *} \quad \uparrow 1.4^{*}$

$\uparrow 1.3^{* *} \quad \mathrm{~ns}$

protein signaling 2)

\section{Hippocampus}

Atpla2 (ATPase,

$$
\downarrow 2.0^{* *} \quad \uparrow 1.5^{* *}
$$

$\uparrow 2.2^{* *}$

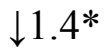

$\mathrm{Na}+/ \mathrm{K}+$ transporting, alpha 2 polypeptide)

$B d n f$ (brain-derived neurotrophic factor)

$$
\uparrow 1.9^{* *} \quad \uparrow 1.4^{* *} \quad \downarrow 1.6^{*} \quad \mathrm{~ns}
$$

$D b p$ (D site albumin

$\uparrow 2.5^{* *} \quad \mathrm{~ns}$

$\uparrow 1.3^{* *} \uparrow 2.4^{* *}$

promoter binding protein)

Grial (glutamate receptor, ionotropic,

\begin{tabular}{|c|c|c|c|c|}
\hline$\downarrow 1.4^{*} \quad \downarrow 1.2^{* *}$ & $\uparrow 1.4 * *$ & ns & - & - \\
\hline - & $\downarrow 2.9^{* *}$ & ns & $\downarrow 1.6^{* *}$ & $\downarrow 1.3^{*}$ \\
\hline
\end{tabular}
AMPA1 (alpha 1)

Homerl (homer homolog 1) 
Table 4-2 (continued)

\begin{tabular}{|c|c|c|c|c|c|c|}
\hline \multirow[b]{2}{*}{ Gene } & \multicolumn{2}{|c|}{ B6 vs. D2 } & \multicolumn{2}{|c|}{ B6, NR vs. R } & \multicolumn{2}{|c|}{ D2, NR vs. R } \\
\hline & Array & qPCR & Array & qPCR & Array & qPCR \\
\hline $\begin{array}{l}N r 4 a 2 \text { (nuclear } \\
\text { receptor subfamily } 4, \\
\text { group } \mathrm{A}, \text { member } 2 \text { ) }\end{array}$ & $\uparrow 1.5^{*}$ & ns & $\downarrow 1.4^{*}$ & $\downarrow 1.4^{* *}$ & - & $1.6^{* *}$ \\
\hline $\begin{array}{l}\text { Rora (RAR-related } \\
\text { orphan receptor } \\
\text { alpha) }\end{array}$ & - & - & $\downarrow 1.3^{* *}$ & ns & - & $1.6^{* *}$ \\
\hline $\begin{array}{l}\text { Per3 (period } \\
\text { homolog } 3)\end{array}$ & - & - & $\uparrow 1.2^{*}$ & $\uparrow 1.4^{*}$ & $\uparrow 1.5^{*}$ & $1.6 * *$ \\
\hline
\end{tabular}


previously implicated in stress-related behavior as well as neural plasticity and circadian regulation.

Our initial strain survey of the effects of repeated restraint stress resulted in a number of important observations. As in previous studies from other laboratories using various repeated stressor, including ten-day restraint, in both rats and mice (e.g., Willner et al., 1996; Pothion et al., 2004; Krishnan et al., 2007; Shansky et al., 2009), we examined relative loss of body weight as an indicator of the efficacy of our stress regimen. As expected, restraint produced a net loss of body weight relative to nonrestrained controls and this was clearly strain-dependent. While, 129S1 mice showed minimal loss, BALB/cByJ and FVBN/J showed the most marked net reduction in body weight, as compared to B6 (which we considered our reference strain).

The 129S1, A/J, BALB/cJ, and BALB/cByJ strains displayed a heightened trait anxiety-like phenotype in the dark/light emergence test under non-restraint basalconditions, as compared to the reference B6 strain, a finding largely consistent with a number of previous studies (for reviews, see Belzung \& Griebel, 2001; Anisman \& Matheson, 2005; Jacobson \& Cryan, 2007; Millstein \& Holmes, 2007). The strong basal phenotype in these strain did, however, prevented the detection of further increases in this behavior as a result of chronic restraint. By contrast, basal anxiety-like behavior in the FVBN/J strain was no different from B6, but unlike B6, FVBN/J mice displayed an anxiogenic-like profile (albeit only on one measure only-shelter exits) following repeated restraint, as compared to non-restrained FVBN/J mice. Of even greater interest, the D2 strain showed both a higher basal level of anxiety-like behavior, as compared to B6, and a further increase in anxiety-like behavior following repeated restraint. Thus, D2 and B6 showed divergent trait and restraint-induced anxiety-like behavior, suggestive of a susceptible and resilient phenotype, respectively. That the B6 strain displayed a relatively stress resilient phenotype concurs with previous reports (for reviews see, Anisman \& Matheson, 2005; Holmes et al., 2005).

In contrast to the marked trait and restraint-induced differences in emergence test anxiety-like behavior between strains, there were fewer clear strain- or restraint-related differences in depression-related behavior as measured via the forced swim test. Perhaps the most notable observation was a decrease in immobility in the BALB/cByJ strain following restraint. A decrease in immobility is similar to that seen with antidepressant treatment (Cryan \& Holmes, 2005) and was therefore an unexpected consequence of restraint, especially in this characteristically high-anxiety and stress-reactive strain (Anisman et al., 2001; Belzung \& Griebel, 2001; Dulawa et al., 2004; Norcross et al., 2008), and additional studies will be needed to clarify the precise nature of this effect.

All strains, including BALB/cByJ, showed robust HPA-axis activation, as measured by serum corticosterone levels, to forced swim stress. Moreover, following repeated restraint, both B6 and D2 showed an augmented HPA-axis response to swim stress, as compared to non-restrained counterparts. This is consistent with a restraintinduced sensitization of the HPA-axis response to a novel stressor in these strains. A similar sensitization was recently found in B6 mice exposed to ten days of social defeat 
(Krishnan et al., 2007). Although none of the other inbred strains we tested showed this effect this may simply have been due to a 'ceiling' level of swim-induced corticosterone obscuring the detection of further increases in the restrained mice. Interestingly however, restrained 129S1 mice actually showed a significantly decreased corticosterone response to swim stress relative to non-restrained counterparts. This finding is intriguing because there is evidence of augmented HPA-axis inhibition in patients with post-traumatic stress disorder (PTSD) (Yehuda, 2002), and we have recently found that the 129S1 strain has an impairment in fear extinction (Hefner et al., 2008); a major symptomatic characteristic of PTSD.

Notwithstanding, the main conclusion from these analyses was that the divergent anxiety-related phenotypes between B6 and D2 did not generalize to measures of depression-related behavior or HPA-axis activation, on which the two strains did not diverge following repeated restraint. Krishnan and colleagues made a similar observation in a study of the effects of repeated social defeat (Krishnan et al., 2007). These authors found that a subgroup of B6 mice that showed decreased body weight and increased social avoidance after repeated defeat did not show abnormalities in forced swim immobility or swim stress-induced corticosterone. Along similar lines, Stekalova et al. (2004) found that C57BL/6N mice exposed to four weeks of a combination of stressors (rat exposure, restraint, tail suspension) had reduced body weight and increased anxietylike behavior (tested in the elevated plus-maze) but normal forced swim performance (Strekalova et al., 2004). Taken together with the current data, these findings indicate that depression-related behavior measured by the forced swim test is insensitive to the effects of repeated stress in most mouse strains.

Genome-wide analysis of gene expression in subpopulations of B6 mice classified as susceptible or resilient to repeated social defeat was recently used as a strategy to identify molecular candidates underlying these differences (Krishnan et al., 2007). We applied a similar strategy to explore the molecular basis of the differences in trait and restraint-induced anxiety-like behavior between B6 and D2 by examining patterns of gene expression in three key regional loci of the corticolimbic circuit mediating stress responses and stress-induced changes in behavior - the $\mathrm{mPFC}$, amygdala, and hippocampus. Consistent with previous studies (Kerns et al., 2005; Grice et al., 2007), our analysis detected substantial expression difference between B6 and D2 with several genes differentially expressed in all three regions.

Amongst the differentially expressed genes confirmed with RT-PCR, B6 had lower amygdala levels of Grinl (encoding the ionotropic N-methyl-D-aspartate (NMDAR) 1 subtype). Interestingly, reduced NMDAR function hs anxiolytic-like effects in rodents (Boyce-Rustay \& Holmes, 2006; Cryan \& Dev, 2007). In addition, B6 had higher hippocampal levels of $B d n f$ (encoding brain-derived neurotrophic factor) and lower levels of Grial (GluR1 AMPA glutamate receptor) than D2. GluR1 is a principal mediator of glutamatergic excitatory neurotransmission (Malenka \& Bear, 2004) and gene knockout of GluR1 produces altered reward and schizophrenia-related disturbances (Bannerman et al., 2006; Wiedholz et al., 2008). A number of interesting strain differences were also found in the $\mathrm{mPFC}$. These included relatively higher mRNA 
expression of Comt (catechol-O-methyltransferase) and lower expression of Chrna4 ( $\alpha 4$ nicotinic receptor) in B6 mice. Both a high COMT activity gene variant (val158met) in humans (Zubieta et al., 2003) and transgenic overexpression of Comt in mice (Papaleo et al., 2008) are associated with reduced stress reactivity. On the other hand, a point mutation causing Chrna4 hypersensitivity increases anxiety-like behavior in mice (Labarca et al., 2001). Of further note, B6 had lower levels of $\operatorname{Rgs} 2$ (regulator of Gprotein signaling 2) in the $\mathrm{mPFC}$. Rgs 2 was recently identified a candidate gene underlying variation in anxiety-like behavior in both mice (Yalcin et al., 2004) and humans (Smoller et al., 2008). Taken together, these basal gene expression differences between B6 and D2 are highly convergent with previous data implicating the same genes in anxiety- and stress-related behaviors, and identify a number of possible candidates underlying the trait differences in anxiety between the two strains.

Repeated restraint produced a pattern of corticolimbic gene expression differences that was very different from that associated with the trait anxiety-related differences between B6 and D2 mice. The amygdala was by far the most stress-sensitive region in terms of number of expression changes produced by restraint, with a lesser although still significant, number of changes in the hippocampus and mPFC. Moreover, in contrast to the considerable regional overlap in expression difference associated with strain, there was a very limited set of genes differentially expressed after restraint in any two regions ( $<$ four percent of the total number of probes). This indicates that restraint drove the expression of quite unique sets of genes in the three regions we examined. An important additional finding was that these restraint-sensitive gene sets were (with some notable exceptions, see below) by and large highly divergent between the B6 and D2 strains; i.e., few of the same expression changes seen in B6 were also seen in D2.

B6 mice showed a significant upregulation of amygdala Grinl (encoding the NMDAR1 glutamate receptor subtype) and a parallel downregulation of Grik1 (kainate 1 glutamate receptor subtype) and Gal (galanin). Numerous studies have pointed to a role for galanin in stress-mediation (Karlsson \& Holmes, 2006), including recent work showing that galanin is upregulated in the ventral tegmental area by repeated social defeat in a 'stress-susceptible' subgroup of C57BL/6J mice (Krishnan et al., 2007).

A striking cluster of gene expression changes associated with repeated restraint involved the circadian genes encoding the period homologs Per 1, Per2 and Per3 and a modulator of their expression, $\mathrm{Dbp}$ (D site albumin promoter binding protein) (Ueda et al., 2005). The diurnal dynamics of these clock genes are modulated by intricate transcriptional and post-translational regulatory loops and are tightly synchronized with glucocorticoid levels (Maywood et al., 2007; Son et al., 2008). Several circadian genes, including Perl, are known to have glucocorticoid response elements in their regulatory regions (Reddy et al., 2007). It is plausible that in the CNS, the persistent stress-induced perturbations in glucocorticoid levels may be mediating the expression changes in the circadian genes. These circadian genes may in turn have downstream effect on the expression of genes such as Homerl and the glutamate receptors (Maret et al., 2007). Interesting, $\mathrm{Nr} 4 \mathrm{a} 2$, a nuclear receptor that interacts directly with the glucocorticoid 
receptor to co-regulate gene expression (Carpentier et al., 2008), was decreased in expression following restraint stress in $\mathrm{C} 57 \mathrm{BL} / 6 \mathrm{~J}$.

Unlike the expression changes discussed above, both strains showed significant upregulation in the clock genes. These changes were particularly interesting in the context of growing evidence linking circadian genes alcoholism and mood disorders (McClung, 2007; Takahashi et al., 2008). For example, mice with knockout of Dbp exhibit locomotor hyperactivity after chronic heterogeneous stress (Le-Niculescu et al., 2008), while Per 2 inactivation increases extracellular levels of glutamate and promotes alcohol drinking (Spanagel et al., 2005), and Perl and Per2 inactivation alters sensitivity to cocaine (Abarca et al., 2002). These data raise the intriguing possibility that repeated restraint might alter the behavioral effects of psychostimulant drugs of abuse and alcohol in mice, as has been found with other forms of repeated stress (Boyce-Rustay et al., 2007).

In summary, the results of the current study provide further evidence of marked strain differences in anxiety-like behavior and identify divergent behavioral effects of repeated stress between two strains, B6 and D2. These differential effects were specific to a measure of anxiety-like behavior and did not generalize to a measure of depressionrelated behavior or sensitization of the HPA-axis to stress. Restraint-induced strain variation in behavior was associated with marked differences in gene expression in corticolimbic brain regions mediating stress-related behaviors. The hippocampus showed that largest number of gene expression differences between B6 and D2 at baseline, while the amygdala was the most sensitive region to repeated restraint. We identified a number of candidate genes underlying differences in anxiety-like behavior between B6 and D2 (e.g., Rgs2, Comt, Grin 1) and the differential response to repeated restraint between the strains (e.g., Grin1, Grik1, Gal). Significant upregulation of circadian clock genes (Perl, Per2, Per3, Dbp) were seen in both strains. 


\section{CHAPTER 5. DISSECTING A QTL HOTSPOT ON MOUSE DISTAL CHROMOSOME 1 THAT MODULATES NEUROBEHAVIORAL PHENOTYPES AND GENE EXPRESSION*}

\subsection{Synopsis}

A remarkably diverse set of traits maps to a region on mouse distal chromosome 1 (Chr 1) that corresponds to human Chr 1q21-q23. This region is highly enriched in quantitative trait loci (QTLs) that control neural and behavioral phenotypes, including motor behavior, escape latency, emotionality, seizure susceptibility (Szsl), and responses to ethanol, caffeine, pentobarbital, and haloperidol. This region also controls the expression of a remarkably large number of genes, including genes that are associated with some of the classical traits that map to distal Chr 1 (e.g., seizure susceptibility). Here we ask whether this QTL-rich region on Chr 1 ( $\mathrm{Qrrl}$ ) consists of a single master locus or a mixture of linked, but functionally unrelated QTLs. To answer this question and to evaluate candidate genes, we generated and analyzed several gene expression, haplotype, and sequence data sets. We exploited six complementary mouse crosses, and combed through 18 expression data sets to determine class membership of genes modulated by Qrrl. Qrrl can be broadly divided into a proximal part ( $\operatorname{rrrlp})$ and a distal part (Qrrld), each associated with the expression of distinct subsets of genes. Qrrld controls RNA metabolism and protein synthesis, including the expression of $\sim 20$ aminoacyl-tRNA synthetases. Qrrld contains a tRNA cluster, and this is a functionally pertinent candidate for the tRNA synthetases. Rgs7 and Fmn2 are other strong candidates in Qrrld. FMN2 protein has pronounced expression in neurons, including in the dendrites, and deletion of Fmn2 had a strong effect on the expression of a subset of genes modulated by Qrrld. Our analysis revealed a highly complex gene expression regulatory interval in $\mathrm{Qrrl}$, composed of multiple loci modulating the expression of functionally cognate sets of genes.

\subsection{Introduction}

The distal part of mouse Chr 1 harbors a large number of QTLs that generate differences in behavior. Open field activity (DeFries et al., 1978), fear conditioning (Caldarone et al., 1997), rearing behavior (Gershenfeld et al., 1997), and several other measures of emotionality (Flint et al., 1995) have been repeatedly mapped to distal Chr 1. This region is also notable because it appears to influence responses to a wide range of drugs including ethanol (Crabbe, 1996), caffeine (Casley et al., 1999), pentobarbital (Buck et al., 1999), and haloperidol (Patel et al., 1999). In addition to the behavioral traits, a number of metabolic, physiological and immunological phenotypes have been mapped to this region (e.g., Beamer et al., 2001; Wang et al., 2004). This QTL rich

* Adapted with permission. Mozhui K, Ciobanu DC, Schikorski T, Wang X, Lu L, Williams RW (2008). Dissection of a QTL hotspot on mouse distal chromosome 1 that modulates neurobehavioral phenotypes and gene expression. PLoS Genet 4:e1000260. 
region on mouse distal $\mathrm{Chr} 1$ exhibits reasonably compelling functional and genetic concordance with the orthologous region on human Chr 1q21-q23. Prime examples of genes in this region that have been associated with similar traits in mouse and human are Rgs2 (anxiety in both species), Apoa2 (atherosclerosis), and Kcnj10 (seizure susceptibility) (Ferraro et al., 2004; Wang et al., 2004; Yalcin et al., 2004; Smoller et al., 2008; Takada et al., 2008).

Studies of gene expression in the central nervous system (CNS) of mice have revealed major strain differences in the expression level of numerous genes located on distal Chr 1, e.g., Copa, Atpla2, and Kcnj9 (Sandberg et al., 2000; Hitzemann et al., 2003; Kerns et al., 2006). These differentially expressed genes are strong candidates for the behavioral and neuropharmacological traits that map to this region. We have recently shown that sequence variants near each of these candidate genes are often responsible for the prominent differences in expression (Hitzemann et al., 2003; Chesler et al., 2005; Peirce et al., 2006). In other words, sequence differences near genes such as Kcnj 9 cause expression to differ, and variation in transcript level maps back to the location of the source gene itself. Transcripts of this type are associated with cis-QTLs.

These expression genetic studies have also uncovered another unusual characteristic of mouse distal $\mathrm{Chr}$ 1. In addition to the extensive cis-effects, a large number of transcripts of genes located on other chromosomes map into this same short interval on distal Chr 1 (Matthews et al., 2005; Peirce et al., 2006). These types of QTLs are often referred to as trans-QTLs. The clustering of trans-QTLs to distal Chr 1 has been replicated in multiple crosses and CNS microarray data sets (Peirce et al., 2006). We refer to this region of Chr 1, extending from $F \operatorname{cgr} 3(172.5 \mathrm{Mb})$ to $\operatorname{Rgs} 7(177.5 \mathrm{Mb})$ as the QTL-rich region on Chr 1, or Qrrl. It is possible that these modulatory effects on expression are the first steps in a cascade of events that are ultimately responsible for many of the prominent differences in behavior and neuropharmacology. For example, Qrrl modulates the expression of several genes that have been implicated in seizure (e.g., Scnlb, Pnpo, Cacnalg), and this may be a basis for the strong influence Qrrl has on seizure susceptibility (Ferraro et al., 2004).

In this study, we exploited 18 diverse array data sets derived from different mouse crosses to systematically dissect the expression QTLs in Qrrl. The strong trans effects are consistently detected in CNS tissues of C57BL/6J (B6) x DBA/2J (D2) and B6 x $\mathrm{C} 3 \mathrm{H} / \mathrm{HeJ}(\mathrm{C} 3 \mathrm{H})$ crosses, but are largely absent in ILS/Ibg (ILS) x ISS/Ibg (ISS) and C57BL/6By (B6y) x BALB/cBy (BALB), and in all non-neural tissues we have examined. We applied high-resolution mapping and haplotype analysis of Qrrl using a large panel of BXD recombinant inbred (RI) strains that included highly recombinant advanced intercross RI lines. Our analyses revealed multiple distinct loci in Qrrl that regulate gene expression specifically in the CNS. The distal part of Qrrl (Qrrld) has a strong effect on the expression of numerous genes involved in RNA metabolism and protein synthesis, including more than half of all aminoacyl-tRNA synthetases. Fmn2 and $\operatorname{Rgs} 7$, and a cluster of tRNAs are the strongest candidates in Qrrld. 


\subsection{Materials and Methods}

\subsubsection{Microarray Datasets}

The microarray data sets used in this study (Table 5-1) were generated by collaborative efforts (Hitzemann et al., 2004; Chesler et al., 2005; Bystrykh et al., 2006; Peirce et al., 2006; Yang et al., 2006; Gatti et al., 2007). All data sets can be accessed from http://www.genenetwork.org. They provide estimates of global mRNA abundance in neural and non-neural tissues in the BXD, LXS, and CXB RI strains, B6D2F2 intercrosses, and B6C3HF2 intercrosses. Detailed description of each set, tissue acquisition, RNA extraction and array hybridization methods, and data processing and normalization methods are provided in the "Info" page linked to each data set. In brief, the data sets are:

1) BXD CNS transcriptomes: The BXD CNS data sets measure gene expression in the forebrain and midbrain (INIA Forebrain), striatum (HBP/Rosen Striatum and HQF Striatum), hippocampus (Hippocampus Consortium and UMUTAffy Hippocampus), cerebellum (SJUT Cerebellum mRNA), and eye (Hamilton Eye) of BXD RI strains. The INIA Brain and HBP/Rosen Striatum data sets have been described in Peirce et al. (2006). The Hippocampus Consortium data set measures gene expression in the adult hippocampus of 69 BXD RI strains, the parental B6 and D2 strains, and F1 hybrids. The SJUT Cerebellum data set measures gene expression in the adult cerebellum of 45 BXD RI strains, parental strains, and F1 hybrids. The Eye dataset measures gene expression in the eyes of $64 \mathrm{BXD}$ RI strains, parental strains, and F1 hybrids. The HQF BXD Striatum is one of the newest data sets and was generated on Illumina Sentrix Mouse-6.1 arrays. It is similar to the HBP/Rosen Striatum and measures gene expression in the striatum of 54 BXD RI strains, parental strains, and F1 hybrids.

2) BXD non-neural transcriptomes: The non-neural $\mathrm{BXD}$ array sets measure gene expression in the liver (UNC Liver) of 40 BXD strains, kidney (Kidney Consortium) of 53 BXD strains, and hematopoietic stem cells (GNF Hematopoietic Cells) of 30 BXD strains (Bystrykh et al., 2006; Gatti et al., 2007).

3) LXS hippocampus transcriptome: The LXS Hippocampus data set measures gene expression in the adult hippocampus of 75 LXS RI strains and the parental ILS and ISS strains.

4) B6D2F2 CNS transcriptomes: The B6D2F2 data sets measure gene expression in the whole brain (OHSU/VA Brain), and striatum (OHSU/VA Striatum) of B6xD2 F2 intercrosses (Hitzemann et al., 2004; Peirce et al., 2006). The whole brain data set comprises of samples from $56 \mathrm{~F} 2$ animals, and the striatum data set comprises of samples from $58 \mathrm{~F} 2$ animals.

5) $\mathrm{B} 6 \mathrm{C} 3 \mathrm{HF} 2$ transcriptomes: These data sets were generated from large numbers of $\mathrm{B} 6 \mathrm{xC} 3 \mathrm{H}$ F2 intercross progeny and assayed using Agilent arrays (Yang et al., 2006). These data sets have been described in Yang et al. (2006). 
Table 5-1 Expression QTLs in Qrr1 in different crosses and tissues

\begin{tabular}{|c|c|c|c|c|c|c|c|c|}
\hline \multirow[b]{2}{*}{ Cross } & \multirow[b]{2}{*}{$\mathrm{N}^{\mathrm{A}}$} & \multirow{2}{*}{$\begin{array}{c}\text { Dataset } \\
\text { Normali- } \\
\text { zation } \\
\end{array}$} & \multirow[b]{2}{*}{ Tissue } & \multirow[b]{2}{*}{ Array } & \multicolumn{4}{|c|}{ LOD $\geq 3$} \\
\hline & & & & & $\mathbf{T}^{\mathbf{B}}$ & $\mathbf{C}^{\mathbf{B}}$ & $\% \mathrm{~T}^{\mathrm{C}}$ & $\% \mathrm{C}^{\mathrm{D}}$ \\
\hline $\mathrm{B} 6 \mathrm{D} 2 \mathrm{~F} 2$ & 58 & $\begin{array}{l}\text { OHSU/VA } \\
\text { (Sep05) } \\
\text { PDNN }\end{array}$ & Striatum & $\begin{array}{l}\text { Affymetrix } \\
\text { M430v2 }\end{array}$ & 197 & 56 & 8 & 5 \\
\hline $\mathrm{B} 6 \mathrm{D} 2 \mathrm{~F} 2$ & 56 & $\begin{array}{l}\text { OHSU/VA } \\
\text { (Aug05) } \\
\text { PDNN }\end{array}$ & $\begin{array}{l}\text { Whole } \\
\text { brain }\end{array}$ & $\begin{array}{l}\text { Affymetrix } \\
\text { M430 }\end{array}$ & 79 & 30 & 1 & 2 \\
\hline BXD & 45 & $\begin{array}{l}\text { SJUT } \\
\text { (Mar05) } \\
\text { PDNN }\end{array}$ & $\begin{array}{l}\text { Cerebel- } \\
\text { lum }\end{array}$ & $\begin{array}{l}\text { Affymetrix } \\
\text { M430 }\end{array}$ & 439 & 44 & 9 & 2 \\
\hline BXD & 69 & $\begin{array}{l}\text { Hippocampus } \\
\text { Consortium } \\
\text { (Dec05) } \\
\text { PDNN }\end{array}$ & $\begin{array}{l}\text { Hippocam- } \\
\text { pus }\end{array}$ & $\begin{array}{l}\text { Affymetrix } \\
\text { M430v2 }\end{array}$ & 345 & 54 & 7 & 1 \\
\hline BXD & 39 & $\begin{array}{l}\text { INIA } \\
\text { (Jan06) } \\
\text { PDNN }\end{array}$ & $\begin{array}{l}\text { Fore- } \\
\text { brain }\end{array}$ & $\begin{array}{l}\text { Affymetrix } \\
\text { M430 }\end{array}$ & 279 & 39 & 5 & 1 \\
\hline BXD & 64 & $\begin{array}{l}\text { Hamilton } \\
\text { Eye } \\
\text { Institute } \\
\text { (Sep06) } \\
\text { RMA }\end{array}$ & Eye & $\begin{array}{l}\text { Affymetrix } \\
\text { M430v2 }\end{array}$ & 156 & 43 & 2 & 1 \\
\hline BXD & 54 & $\begin{array}{l}\text { HQF } \\
\text { (Nov 07) } \\
\text { RankInv }\end{array}$ & Striatum & $\begin{array}{l}\text { Illumina } \\
\text { M6.1 }\end{array}$ & 97 & 31 & 1 & 1 \\
\hline BXD & 29 & $\begin{array}{l}\text { HBP/Rosen } \\
\text { (Apr05) } \\
\text { PDNN }\end{array}$ & Striatum & $\begin{array}{l}\text { Affymetrix } \\
\text { M430v2 }\end{array}$ & 94 & 25 & 2 & 1 \\
\hline BXD & 63 & $\begin{array}{l}\text { UMUTAffy } \\
\text { (Mar08) } \\
\text { RMA }\end{array}$ & $\begin{array}{l}\text { Hippocam- } \\
\text { pus }\end{array}$ & $\begin{array}{l}\text { Affymetrix } \\
\text { Exon } 1.0 \\
\text { ST }\end{array}$ & 700 & 302 & 0.4 & 1 \\
\hline BXD & 40 & $\begin{array}{l}\text { UNC } \\
\text { (Jan06) } \\
\text { LOWESS }\end{array}$ & Liver & $\begin{array}{l}\text { Agilent } \\
\text { G4121A }\end{array}$ & 9 & 20 & 0.3 & 1 \\
\hline BXD & 53 & $\begin{array}{l}\text { Kidney } \\
\text { Consortium } \\
\text { (Aug06) } \\
\text { PDNN }\end{array}$ & Kidney & $\begin{array}{l}\text { Affymetrix } \\
\text { M430v2 }\end{array}$ & 8 & 33 & 0.2 & 1 \\
\hline
\end{tabular}


Table 5-1 (continued)

\begin{tabular}{|c|c|c|c|c|c|c|c|c|}
\hline \multirow[b]{2}{*}{ Cross } & \multirow[b]{2}{*}{$\mathbf{N}^{\mathbf{A}}$} & \multirow{2}{*}{$\begin{array}{l}\text { Dataset } \\
\text { Normali- } \\
\text { zation }\end{array}$} & \multirow[b]{2}{*}{ Tissue } & \multirow[b]{2}{*}{ Array } & \multicolumn{4}{|c|}{ LOD $\geq 3$} \\
\hline & & & & & $\mathbf{T}^{\mathbf{B}}$ & $\mathbf{C}^{\mathbf{B}}$ & $\% \mathrm{~T}^{\mathrm{C}}$ & $\% \mathrm{C}^{\mathrm{D}}$ \\
\hline BXD & 30 & $\begin{array}{l}\text { GNF } \\
\text { (Mar03) } \\
\text { MAS5 }\end{array}$ & $\begin{array}{l}\text { Hemato- } \\
\text { poietic } \\
\text { Cells }\end{array}$ & $\begin{array}{l}\text { Affymetrix } \\
\text { U74Av2 }\end{array}$ & 0 & 6 & 0 & 3 \\
\hline LXS & 75 & $\begin{array}{l}\text { NIAAA } \\
\text { INIA } \\
\text { (May07) } \\
\text { RankInv }\end{array}$ & $\begin{array}{l}\text { Hippocam- } \\
\text { pus }\end{array}$ & $\begin{array}{l}\text { Illumina } \\
\text { M6.1 }\end{array}$ & 10 & 28 & 0.4 & 1 \\
\hline $\mathrm{B} 6 \mathrm{C} 3 \mathrm{~F} 2$ & 238 & $\begin{array}{l}\text { UCLA } \\
\text { (2005) } \\
\text { mlratio }\end{array}$ & Brain & Agilent & 516 & 51 & 14 & 3 \\
\hline $\mathrm{B} 6 \mathrm{C} 3 \mathrm{~F} 2$ & 306 & $\begin{array}{l}\text { UCLA } \\
\text { (2005) } \\
\text { mlratio }\end{array}$ & Muscle & Agilent & 15 & 33 & 0.3 & 2 \\
\hline $\mathrm{B} 6 \mathrm{C} 3 \mathrm{~F} 2$ & 298 & $\begin{array}{l}\text { UCLA } \\
\text { (2005) } \\
\text { mlratio }\end{array}$ & Liver & Agilent & 63 & 46 & 0.7 & 3 \\
\hline $\mathrm{B} 6 \mathrm{C} 3 \mathrm{~F} 2$ & 282 & $\begin{array}{l}\text { UCLA } \\
(2005) \\
\text { mlratio }\end{array}$ & Adipose & Agilent & 56 & 34 & 0.5 & 3 \\
\hline CXB & 13 & $\begin{array}{l}\text { Hippocampus } \\
\text { Consortium } \\
\text { (Dec05) } \\
\text { PDNN }\end{array}$ & $\begin{array}{l}\text { Hippocam- } \\
\text { pus }\end{array}$ & $\begin{array}{l}\text { Affymetrix } \\
\text { M430v2 }\end{array}$ & 7 & 12 & 0.08 & 2 \\
\hline
\end{tabular}

${ }^{A}$ Number of RI strains or F2 mice; ${ }^{\mathrm{B}}$ Number of trans-QTLs (T) and cis-QTLs (C); ${ }^{\mathrm{C}}$ Percent of trans-QTLs in Qrr1 = [(number of trans-QTLs in Qrr1)/(total number of

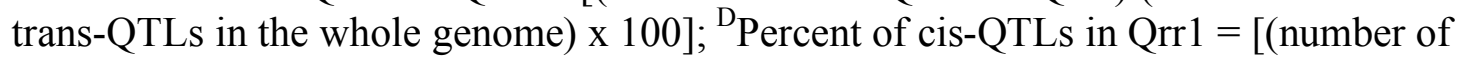
cis-QTLs in Qrr1)/(total number of cis-QTLs in the whole genome) $\mathrm{x} 100]$. 


\subsubsection{Mouse Strains and Genotype Data}

The conventional BXD RI strains were derived from the B6 and D2 inbred mice (Taylor, 1989; Taylor et al., 1999). The newer sets of advanced RI strains were derived by inbreeding intercrosses of the RI strains (Peirce et al., 2004). The parental B6 and D2 strains differ significantly in sequence and have approximately 2 million informative SNP. A subset of 14,000 SNPs and microsatellite markers have been used to genotype the BXD strains (Williams et al., 2001b; Shifman et al., 2006). We used 3,795 informative markers for QTL mapping. Thirty such informative markers are in Qrr1 and we queried these markers to identify strains with recombinations in Qrrl; genes with strong cisQTLs (Sdhc, Atpla2, Dfy, and Fmn2) were used as additional markers. Smaller sub-sets of markers were used to genotype the two F2 panels (total of 306 markers for the whole brain, and 75 markers for the striatum F2 datasets).

The LXS RI strains were derived from the ILS and ISS inbred strains. They have been genotyped using 13,377 SNPs, and some microsatellite markers (Williams et al., 2004). 2,659 informative SNPs and microsatellite markers were used for QTL mapping. The CXB panel consists of $13 \mathrm{RI}$ strains derived from C57BL/6By and BALB/cBy inbred strains. A total of 1384 informative markers were used for QTL mapping.

The $\mathrm{B} 6$ x C3H/HeJ F2 intercrosses have been genotyped using 13,377 SNPs and microsatellite markers, and 8,311 informative markers were used for QTL mapping.

\subsubsection{Animals and Tissue Acquisition}

Majority of the BXD and LXS tissues (cerebellum, eye, forebrain, hippocampus, kidney, liver, and striatum for the HQF Illumina data set) were dissected at the University of Tennessee Health Science Center (UTHSC). Mice were housed at the UTHSC in pathogen-free colonies, at an average of three mice per cage. All animal procedures were approved by the Animal Care and Use Committee. Mice were killed by cervical dislocation, and tissues were rapidly dissected and placed in RNAlater (Ambion, http://www.ambion.com) and kept overnight at $4^{\circ} \mathrm{C}$, and subsequently stored at $-80^{\circ} \mathrm{C}$. Tissue were then processed at UTHSC or shipped to other locations for processing.

\subsubsection{RNA Isolation and Sample Preparation}

For the tissues that were processed at UTHSC (all BXD and LXS CNS tissues except HBP Affymetrix striatum), RNA was isolated using RNA STAT-60 (Tel-Test Inc., http://www.tel-test.com) as per manufacturer's instructions. Samples were then purified using standard sodium acetate methods prior to microarray hybridization. The eye samples required additional purification steps to remove eye pigment; this was done using the RNeasy MinElute Cleanup Kit (Qiagen, http://www.qiagen.com). RNA purity and concentration was evaluated with a spectrophotometer using $260 / 280 \mathrm{~nm}$ absorbance 
ratio, and RNA quality was checked using Agilent Bioanalyzer 2100 prior to hybridization. Array hybridizations were then done according to standard protocols.

\subsubsection{Microarray Probe Set Annotation}

We have re-annotated a majority of Affymetrix probe sets to ensure more accurate description of probe targets. Each probe set represents a concatenations of eleven 25-mer probes, and these have been aligned to the NCBI built 36 version of the mouse genome (mm8 in UCSC Genome Browser) by BLAT analysis. We have also re-annotated the Illumina probes and incorporated these annotations into GeneNetwork. Each probe in the Illumina Mouse-6 and Mouse-6.1 arrays is 50 nucleotides in length, and these have been aligned to NCBI built 36.

\subsubsection{QTL Mapping}

We used the strain average expression signal detected by a probe or probe set. QTL mapping was done for all transcripts using QTL Reaper (Peirce et al., 2006). QTL Reaper performs up to a million permutations of an expression trait to calculate the genome-wide empirical $p$ and the LOD score associated with a marker. We selected only those transcripts that have highest LOD scores, i.e., genome-wide adjusted best $p$-values, on markers located on Chr 1 from 172 to $178 \mathrm{Mb}$. This selected transcripts that are primarily modulated by Qrrl but excluded transcripts that have QTLs in Qrrl but have higher LOD scores on markers located on other chromosomal regions. Cis- and transQTLs were distinguished based on criteria described by Peirce et al. (2006). To identify trans-QTLs common to multiple data sets, we selected probes/probe sets that target the same genes and have peak LOD scores within $10 \mathrm{Mb}$ in the different data sets.

\subsubsection{Screening Local QTLs}

We screened all Affymetrix probe sets with cis-QTLs in Qrr1 for SNPs in target sequences. This step was taken to identity false cis-QTLs caused by differences in hybridization. As probe design is based on the B6 sequence, such spurious cis-QTLs show high expression for the $B$ allele, and low expression for the $D$ allele. Our screening identified only two probe sets in which SNPs result in spurious local QTLs-1429382_at (Tomm40l), and 1452308_a_at (Atpla2). The majority of cis-QTLs in Qrrl are likely to be due to actual differences in mRNA abundance. We did not detect a bias in favor of the $\mathrm{B}$ allele on cis-regulated expression and the ratio of transcripts with B- and D- positive additive effects is close to $1: 1$. 


\subsubsection{Analysis of Allele Specific Expression Difference}

To measure expression difference between the B and D alleles, we exploited transcribed SNPs to capture allelic expression difference in F1 hybrids (Cowles et al., 2006) using a combination of RT-PCR and a single base extension technology (SNaPshot, Applied Biosystems, http://www.appliedbiosystems.com). For each transcript we analyzed, Primer 3 (Rozen et al., 2000) was used to design a pair of PCR primers that target sequences on the same exon and flanking an informative SNP.

We prepared four pools of RNA from the hippocampus, and four pools of genomic DNA from the spleen of F1 hybrids (male and female B6xD2 and D2xB6 F1 hybrids). To avoid contamination by genomic DNA, the four RNA pools were treated with Turbo DNase (Ambion, http://www.ambion.com), and then first strand cDNA was synthesized (GE Healthcare, http://www.gehealthcare.com). The genomic DNA samples were used as controls, and both cDNA and genomic DNA samples were tested concurrently using the same assay to compare expression levels of $B$ and $D$ transcripts.

We amplified the cDNA and genomic DNA samples using GoTaq Flexi DNA polymerase (Promega Corporation, http://www.promega.com). PCR products were purified using ExoSap-IT (USB Corporation, http://www.usbweb.com) followed by SNaPshot to extend primer by a single fluorescently labeled ddNTPs. Fluorescently labeled products were purified using calf intestinal phosphatase (CIP, New England BioLabs, http://www.neb.com) and separated by capillary electrophoresis on ABI3130 (Applied Biosystems). Quantification was done using GeneMapper v4.0 software (Applied Biosystems), and transcript abundance was measured by peak intensities associated with each allele. Ratio of $B$ and $D$ allele in both cDNA and gDNA pools was computed, and t-test (one tail, unequal variance) was done to validate expression difference and polarity of parental alleles.

\subsubsection{SNP Analysis in Multiple Crosses}

GeneNetwork has compiled SNP data from different sources - Celera (http://www.celera.com), Perlegen/NIEHS (http://mouse.perlegen.com/mouse/download.html), BROAD institute (http://www.broad.mit.edu/snp/mouse), Wellcome-CTC (Shifman et al., 2006), dbSNP, and Mouse Phenome Database (http://www.jax.org/phenome/SNP). SNP counts were done on the GeneNetwork SNP browser.

\subsubsection{Partial Correlation Analysis}

A partial correlation is the correlation between $X$ and $Y$ conditioned on one or more control variables. In this study, first order partial correlation was used to detect the interaction between trans-regulated transcripts and cis-regulated candidate genes conditioned on the genotype (marker rs8242481 at $175.058 \mathrm{Mb}$ ). If $\mathrm{x}, \mathrm{y}$ and $\mathrm{z}$ are trans- 
regulated transcripts, cis-regulated transcript, and genotype in the QTL, respectively, then the first order partial correlation coefficient is calculated as -

$$
r_{x y . z}=\frac{r_{x y}-r_{x z} r_{y z}}{\sqrt{\left(1-r_{x z}^{2}\right)\left(1-r_{y z}^{2}\right)}}
$$

where $r_{x y}$ can be either Pearson correlation or Spearman's rank correlation between $\mathrm{x}$ and y. We employed the Spearman's rank correlation because the expression levels of many transcripts do not follow a normal distribution. The significance of a partial correlation with $n$ data points was assessed with a two-tailed $t$ test-

$$
t=r \sqrt{\frac{n-2-k}{1-r^{2}}} \sim t_{n-2-k}
$$

where $r$ is the first order correlation coefficient, and $k$ is the number of variables on which we are conditioning.

\subsubsection{Immunocytochemistry}

Cultured hippocampal neurons from male B6 mice, prepared as described in Schikorski et al. (2007) and cultured for 23 days, were fixed with 4\% paraformaldehyde and $0.1 \%$ glutaraldehyde in HEPES buffered saline (pH7.2) for 15 min. Cell membranes were permeabilized with $0.1 \%$ triton X-100 and unspecific binding sites were quenched with $10 \%$ BSA for $20 \mathrm{~min}$ at room temperature (RT). Neurons were incubated with a polyclonal anti-FMN2 antibody (Protein Tech Group, http://www.ptglab.com) diluted to $0.3 \mu \mathrm{g} / \mathrm{ml}$ at RT overnight. An anti-rabbit antibody raised in donkey (1:500, http://www.invitrogen.com) conjugated with the fluorescent dye Alexa488 was used for the detection of the first antibody. All regions of interest were photographed with identical illumination and camera settings to allow for a direct comparison of the staining in labeled and control neurons.

\subsubsection{Microarray Analysis of Fmn2-Knockout Mice}

The Fmn2 $2^{-/}$mice were generated using 129/SvEv (now strain 129S6/SvEvTac) derived TC-1 embryonic stem cells. Chimeric mice were backcrossed to $129 / \mathrm{SvEv}$ (Leader et al., 2000). The Fmn2-null and littermate controls are therefore coisogenic. To validate the isogenicity of regions surrounding the targeted locus (Gerlai, 1996), we genotyped the $\mathrm{Fmn2}^{+/+}, \mathrm{Fmn}^{+/-}$, and $\mathrm{Fmn}^{-/-}$mice using ten microsatellite markers located on, and flanking Fmn2 (markers distributed from $172 \mathrm{Mb}$ to $182 \mathrm{Mb}$ ). These markers are D1Mit455, D1Mit113, D1Mit456, D1Mit356, D1Mit206, D1Mit355, D1Mit150, D1Mit403,D1Mit315, and D1Mit426. With the exception of a marker at Fmn2 (D1Mit150), all alleles in null, heterozygote, and wildtype animals were identical. 
RNA was isolated from whole brain samples of $F m n 2^{+/+}$and $F m n 2^{-/-}$mice, and assayed on Illumina Mouse-6 array slides (six samples per slide). We compared five samples from $\mathrm{Fmn} 2^{-/-}$nulls, and five samples from $\mathrm{Fmn}^{+/+}$wildtype. Equal numbers of each genotypes were placed on each slide to avoid batch confounds. Microarray data were processed using both raw and rank invariant protocols provided by Illumina as part of the BeadStation software suite (http://www.illumina.com). We subsequently log-transformed expression values and stabilized the variance of each array. To identify genes with significant expression difference between the $\mathrm{Fmn}^{-/-}$and $\mathrm{Fmn}^{+/+}$cases, we carried out two-tailed $t$-tests and applied a Bonferroni correction for multiple testing, and selected probes with a minimum adjusted $p<0.05$.

\subsubsection{Bioinformatics Tools}

The April 2008 version of Mouse Genome Informatics was used to survey classical QTLs in Qrrl (MGI: http://www.informatics.jax.org) (Eppig et al., 2005). Search for tRNAs was done using tRNAscan-SE 1.21 (http://lowelab.ucsc.edu/tRNAscan-SE/) (Lowe et al., 1997). GO analysis was done using the analytical tool DAVID 2007 (http://david.abcc.ncifcrf.gov/) (Dennis et al., 2003). Overrepresented GO terms were identified and statistical significance of enrichment was calculated using a modified Fisher's Exact Test or EASE score (Hosack et al., 2003). We used the Allen Brain Atlas to analyze expression pattern in the brain of young C57BL/6J male mice (http://www.brain-map.org) (Lein et al., 2007; Lau et al., 2008).

\subsubsection{Control for Non-Syntenic Association and Paralogous Regions}

In RI strains, non-syntenic associations can lead to LD between distant loci (Petkov et al., 2006; Williams et al., 2001b). In the BXDs, we detected such non-syntenic associations between markers in Qrrl and markers on distal Chr 2 and proximal Chr 15. As a result of these associations, some transcripts that have strong cis- or trans-QTLs in Qrrl tend to have weak LOD peaks, usually below the suggestive threshold, on distal Chr 2 and proximal Ch15. However, there is no bias for genes located in these intervals in LD with Qrrl to have trans-QTLs in Qrrl.

The Qrrl segment has been reported to have paralogues on mouse Chrs 1 (proximal region), 2, 3, 6, 7, 9, and 17 (Katsanis et al., 1996; Stanier et al., 1998). We examined if the trans-QTLs in Qrrl are of genes located in these paralogous regions. However, genes located in the paralogous regions are not overrepresented among the trans-QTL. 


\section{$5.4 \quad$ Results}

\subsubsection{Enrichment in Expression QTLs in Neural Tissues}

In this section, we summarize the number of expression phenotypes that map to Qrrl in different tissues and mouse crosses. The results are based on the analysis of 18 array data sets that provide estimates of global mRNA abundance in neural and nonneural tissues from six different crosses. These crosses are-(i) BXD RI and advanced intercross RI strains derived from B6 and D2, (ii) CXB RI strains derived from B6y x BALB, (iii) LXS RI strains derived from ILS and ISS, (iv) B6xC3H F2 intercrosses, and (v \& vi) two separate B6xD2 F2 intercrosses. These data sets were generated by collaborative efforts over the last few years (Hitzemann et al., 2004; Bystrykh et al., 2005; Chesler et al., 2005; Peirce et al., 2006; Yang et al., 2006; Gatti et al., 2007) and some were generated more recently (e.g., the Illumina data sets for BXD striatum and LXS hippocampus, and BXD Hippocampus UMUTAffy Exon Array data set). All data sets can be accessed from GeneNetwork (http://www.genenetwork.org).

We mapped loci that modulate transcript levels and selected only those transcripts that have peak QTLs in Qrrl with a minimum LOD score of 3. This corresponds to a generally lenient threshold with genome-wide $p$ of 0.1 to 0.05 , but corresponds to a highly significant pointwise $p$. Because we are mainly interested in testing a short segment on Chr 1, a pointwise (region-wise) threshold is more appropriate to select those transcripts that are likely to be modulated by Qrr 1. Qrrl covers approximately $0.2 \%$ of the genome and extends from Fcgr3 (more precisely, SNP rs8242852 at $172.887364 \mathrm{Mb}$ using Mouse Genome Assembly NCBI m36, UCSC Genome Browser mm8) through to Rgs7 (SNP rs4136041 at 177.273526 Mb). We defined this region on the basis of the large number of transcripts that have maximal LOD scores associated with markers between these SNPs.

Hundreds of transcripts map to Qrrl with LOD scores $\geq 3$ in neural tissue data sets of BXD RI strains, B6D2F2 intercrosses, and B6C3HF2 intercrosses (Table 5-1). The QTL counts in Qrrl are far higher than the average of 15 to 35 expression QTLs in a typical $6 \mathrm{Mb}$ interval. The fraction of QTLs in Qrrl is as high as 14\% of all trans-QTLs, and 5\% of all cis-QTLs in the whole genome. The enrichment in trans-QTLs in Qrrl is even more pronounced when the QTL selection stringency is increased to a LOD threshold of 4 (genome-wide $p$ of approximately 0.01 ). For example, $27 \%$ of all highly significant trans-QTLs in the BXD cerebellum data set are in Qrrl. The BXD hippocampus data set that was assayed on the Affymetrix Exon ST array is an exception-there are over a million probe sets in this array, and the percent enrichment of QTLs in Qrrl appears to be relatively low. Nevertheless, about 1000 transcripts map to Qrrl in this exon data set.

In contrast to the CNS data sets, relatively few transcripts map to Qrrl in nonneural tissues of the BXD strains and B6C3HF2 intercrosses. While the number of cis- 
QTLs is still relatively high (1-3\%), Qrrl has limited or no trans-effect in these data sets (Table 5-1).

Qrr1 does not have a strong trans-effect in the LXS and CXB hippocampus data sets (Table 5-1). This indicates that the sequence variants underlying the trans-QTLs do not segregate to nearly the same extent in the LXS and CXB RI panels as they do in $\mathrm{B} 6 \mathrm{xD} 2$ and $\mathrm{B} 6 \mathrm{xC} 3 \mathrm{H}$ crosses. This contrast among crosses can be exploited to parse Qrr 1 into sub-regions and identify stronger candidate genes.

\subsubsection{Replication of Trans-QTLs in Multiple Data Sets}

The trans-QTLs in Qrr1 are highly replicable. A large fraction of the transcripts, in some cases represented by multiple probes or probe sets, map to Qrrl in multiple CNS data sets. For example, there are 747 unique trans-QTLs with LOD scores greater than 4 (genome-wide $p \leq 0.01$ ) in the BXD hippocampus data set (assayed on Affymetrix M430v2 arrays). Out of these highly significant trans-QTLs, 155 are in Qrrl and the remaining 592 are distributed across the rest of the genome (Figure 5-1). We compared the trans-QTLs in the hippocampus data set with a similar collection of trans-QTLs $(\mathrm{LOD} \geq 4)$ in the cerebellum data set (assayed on Affymetrix M430 arrays). Only 101 trans-QTLs in the hippocampus are replicated in the cerebellum (for trans-QTLs that were declared as common, the average distance between peak QTL markers in the two data sets is $1.6 \mathrm{Mb}$ ). But it is remarkable that of the subset of common trans-QTLs, 64 are in $\operatorname{Qrrl}$ (Figure 5-1). The replication rate of trans-QTLs in Qrrl is therefore about 6fold higher relative to the rest of the genome. When we compared the BXD hippocampus data set with the B6C3HF2 brain dataset (assayed on Agilent arrays), we found 54 transQTLs common to both data sets (for the common trans-QTLs, the average distance between peak markers in the two data sets is $2.7 \mathrm{Mb}$ ). Strikingly, out of the 54 transQTLs common to both crosses, 52 are in $\operatorname{Vrrl}$ (Figure 5-1).

Among the transcripts with the most consistent trans-QTLs are glycyl-tRNA synthetase (Gars), cysteinyl-tRNA synthetase (Cars), asparaginyl-tRNA synthetase (Nars), isoleucyl tRNA synthetase (Iars), asparagine synthetase (Asns), and activating transcription factor 4 (Atf4). These transcripts map to Qrrl in almost all data sets in which the strong trans-effect is detected. Gars, Cars, and Nars are aminoacyl-tRNA synthetases (ARS) that charge tRNAs with amino acids during translation. Asns and Atf 4 are also involved in amino acid metabolism-Asns is required for asparagines synthesis and is under the regulation of Atf4, which in turn is sensitive to cellular amino acid levels (Sui et al., 2002). Other transcripts that consistently map as trans-QTLs to Qrrl include brain expressed X-linked 2 (Bex2), splicing factor Sfrs3, ribonucleoproteins Snrpc and Snrpd1, ring finger protein 6 (Rnf6), and RAS oncogene family member Rab2. 


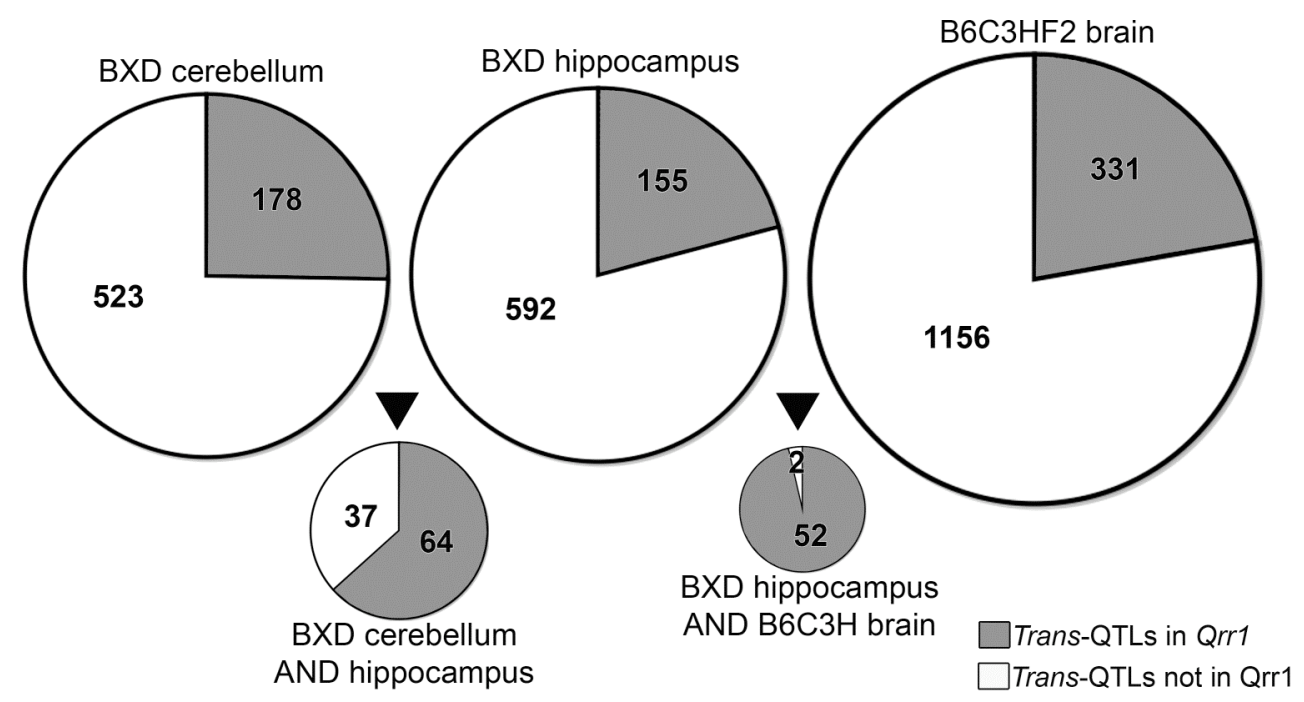

Figure 5-1 Highly replicable trans-QTLs in Qrr1

The charts illustrate the total number of trans-QTLs (LOD $\geq 4$ ) in Qrrl (shaded) and in other regions of the genome (non-shaded) in three data sets-BXD cerebellum, BXD hippocampus, and $\mathrm{B} 6 \mathrm{C} 3 \mathrm{H} \mathrm{F} 2$ brain. The smaller charts represent the trans-QTLs in BXD hippocampus that are also detected in BXD cerebellum, and B6C3HF2 brain data sets. Out of the 101 trans-QTLs common to both BXD hippocampus and cerebellum, 64 are in Qrrl and the remaining 37 are located in other regions of the genome. The BXD hippocampus and B6C3HF2 brain data sets have 54 common trans-QTLs, and almost all (52 out of 54) are in Qrrl. 


\subsubsection{Candidates in Qrr1}

Qrrl contains 164 known genes. The proximal part of Qrrl is gene-rich and has several genes with high expression in the CNS (e.g. Pea15, Kcnj9, Kcnj10, Atpla2). The middle to distal part of Qrrl is relatively gene sparse and consists mostly of clusters of olfactory receptors and members of the interferon activated Ifi200 gene family. Though comparatively gene sparse, the middle to distal part of Qrrl contains a small number of genes that have high expression in the CNS - Igsf4b, Dfy, Fmn2, and Rgs 7 .

A subset of 35 genes were initially selected as high priority candidates based on the number of known and inferred sequence differences between the B6 allele $(B)$ and D2 allele $(D)$ and based on expression levels in multiple CNS data sets (Table 5-2). Eleven of these candidates contain missense SNPs segregating in B6xD2 crosses. We also scanned Qrrl for variation in copy number (Li et al., 2004; Graubert et al., 2007). Graubert et al. (2007) reported segmental duplication in Qrrl with a copy number gain in D2 compared to B6 near the intelectin $1($ It $\ln a)$ gene at $173.352 \mathrm{Mb}$. We failed to detect any expression signatures of a copy number variation around Itlna in any of the GeneNetwork data sets. However, we did identify an apparent $150 \mathrm{~kb}$ deletion across the Ifi200 gene cluster (175.584-175.733 Mb). Affymetrix probe sets 1426906 at, 1452231_x_at, and 1452349_x_at detect Ifi204 and Mnda transcripts in B6 but not in D2. The expression difference is robust enough to generate cis-QTLs with very high LOD scores $(>40)$. This gene cluster has low expression in the CNS (Affymetrix declares this probe sets to be "not present"), but high expression in tissues such as hematopoietic stem cells and kidney, in which the trans-effect of Qrrl is not detected. The Ifi200 gene cluster was therefore excluded as a high priority candidate.

\subsubsection{Cis-QTLs in Qrr1}

Transcripts of 26 of the 35 selected candidate genes map as cis-QTLs (LOD $\geq 3$ ) in the BXD CNS data sets (Table 5-2). These putatively cis-regulated genes are among the strongest candidates in the QTL interval. The $D$ allele in $\operatorname{Qrr} 1$ has the positive effect on the expression of Sdhc, Ndufs2, Adamts4, Dedd, Pfdn2, Ltap, Pea15, Atpla2, Kcnj9, $K c n j 10, \operatorname{Igsf4b}$, and Grem2. Increase in expression caused by the $D$ allele ranges from about $10 \%$ for Adamts 4 to over 2-fold for Atpla2. In contrast, the $B$ allele has the positive effect on the expression of Pcp4l1, Fcerlg, B4galt3, Ppox, Ufc1, Nit1, Usf1, Copa, Pex 19, Wdr42a, Igsf8, Dfy, Fmn2, and Rgs7. Increase in expression caused by the $B$ allele ranges from about $7 \%$ for $U s f 1$ to $40 \%$ for Pex 19 .

Individual probes were screened to assess if the strong cis-effects are due to hybridization artifacts caused by SNPs in probe targets. Thirteen candidate genes with cis-QTLs were then selected for further analysis and validation of cis-regulation by measuring allele specific expression (ASE) difference (Cowles et al., 2002). This method exploits transcribed SNPs, and uses single base extension to assess expression difference in F1 hybrids. By means of ASE, we validated the cis-regulation of 10 candidate genesNdufs2, Nit1, Pfdn2, Usf1, Copa, Atpla2, Kcnj9, Kcnj10, Dfy, and Fmn2 (Table 5-3). 
Table 5-2 Candidate genes in Qrr1

\begin{tabular}{|c|c|c|c|c|c|c|}
\hline Gene & SNP $^{A}$ & $\operatorname{Exp}^{B}$ & $\mathbf{B X D}^{\mathrm{C}}$ & $\begin{array}{l}\text { B6C3H } \\
\text { F2 }^{\text {C }} \\
\end{array}$ & $\mathrm{CXB}^{\mathrm{C}}$ & $\mathbf{L X S}^{\mathrm{C}}$ \\
\hline Fcgr 3 & 2 & 8.2 & & & & $c i s$ \\
\hline$S d h c$ & 2 & 12.3 & cis & & & cis \\
\hline Pcp $4 l 1$ & & 8.7 & $c i s$ & cis & & \\
\hline Tomm40l & & 9.67 & $c i s$ & & $c i s$ & \\
\hline Apoa 2 & & 7.2 & & cis & $c i s$ & cis \\
\hline Fcerlg & & 8.5 & cis & & & cis \\
\hline$N d u f_{s} 2$ & 2 & 13.6 & cis & & & \\
\hline Adamts4 & 1 & 8.1 & $c i s$ & cis & $c i s$ & cis \\
\hline B4galt3 & & 9.5 & cis & & & \\
\hline Pрох & & 7.8 & cis & cis & & cis \\
\hline Usp21 & & 9.0 & & & & cis \\
\hline Uf & & 10.8 & $c i s$ & $c i s$ & $c i s$ & $c i s$ \\
\hline Dedd & & 9.7 & cis & & & \\
\hline Nit1 & 1 & 9.8 & cis & cis & cis & cis \\
\hline$P f d n 2$ & & 12.8 & $c i s$ & cis & cis & \\
\hline Arhgap 30 & 4 & 7.6 & & & & \\
\hline Usf1 & & 7.5 & cis & cis & & cis \\
\hline Refbp 2 & 2 & 9.7 & & cis & & $c i s$ \\
\hline Vangl2 & & 7.6 & cis & cis & cis & cis \\
\hline Ncstn & & 8.5 & & cis & & cis \\
\hline Сора & 1 & 12.7 & $c i s$ & $c i s$ & & $c i s$ \\
\hline Pex19 & 1 & 9.9 & $c i s$ & & $c i s$ & cis \\
\hline$W d r 42 a$ & & 10.3 & cis & cis & & \\
\hline Peal5 & & 14.1 & & cis & & \\
\hline Atpla2 & & 15.4 & $c i s$ & cis & $c i s$ & $c i s$ \\
\hline $\operatorname{Igsf} 8$ & & 12.1 & $c i s$ & & & \\
\hline Kcnj9 & & 9.1 & $c i s$ & cis & cis & $c i s$ \\
\hline Kcnj10 & 1 & 11.2 & cis & cis & cis & \\
\hline Tagln 2 & & 8.8 & & & & \\
\hline Dusp23 & & 7.4 & & cis & & \\
\hline Dfy & & 10.3 & $c i s$ & & cis & cis \\
\hline $\operatorname{Igs} f 4 b$ & & 10.6 & $c i s$ & & & \\
\hline Fmn2 & 3 & 10.4 & cis & cis & cis & \\
\hline Grem 2 & & 8.2 & $c i s$ & & & \\
\hline $\operatorname{Rgs} 7$ & & 11.5 & cis & cis & & \\
\hline
\end{tabular}


Table 5-3 Validation of cis-QTLs

\begin{tabular}{|c|c|c|c|c|c|c|}
\hline Gene & ProbeSet ID & SNP ID & $\begin{array}{l}\text { Cis- } \\
\text { LOD }\end{array}$ & $\begin{array}{c}\text { Add. } \\
\text { Effect } \\
\text { (QTL) }^{\mathbf{A}}\end{array}$ & $\begin{array}{c}\text { High } \\
\text { Allele } \\
\text { (ASE) }\end{array}$ & $\boldsymbol{P}$ \\
\hline$N d u f_{s} 2$ & 1451096_at & rs8245216 & 12 & 0.172 & $D$ & $2.4 \times 10^{-5}$ \\
\hline Adamts4 & 1455965_at & rs31537832 & 25 & -0.376 & & 0.2 \\
\hline$U f_{c} l$ & 1416327_at & rs 13470410 & 21 & -0.262 & $D$ & 0.02 \\
\hline Nit1 & 1417468_at & rs31552469 & 15 & -0.154 & $B$ & 0.01 \\
\hline$P f d n 2$ & 1421950_at & rs31549998 & 5 & 0.174 & $D$ & $4.1 \times 10^{-7}$ \\
\hline$U_{s f 1}$ & 1426164_a_at & rs31542370 & 5 & -0.166 & $B$ & 0.004 \\
\hline Copa & 1415706_at & rs13461812 & 9 & -0.148 & $B$ & $3.9 \times 10^{-5}$ \\
\hline Atpla2 & 1455136 at & rs31570902 & 49 & 1.186 & $D$ & 0.02 \\
\hline Kcnj9 & 1450712_at & rs31569118 & 19 & 0.511 & $D$ & 0.01 \\
\hline Kcnj10 & 1419601_at & rs30789204 & 28 & 0.349 & $D$ & 0.003 \\
\hline Dfy & 1432273_a_at & rs31616337 & 24 & -0.337 & $B$ & 0.006 \\
\hline $\operatorname{Igs} f 4 b$ & 1418921_at & rs31613626 & 7 & 0.171 & & 0.3 \\
\hline Fmn2 & 1450063 at & rs33800912 & 17 & -0.286 & $B$ & $5.5 \times 10^{-6}$ \\
\hline
\end{tabular}

${ }^{\mathbf{A}}$ Additive effect is computed as [(mean expression in $D D$ homozygote) - (mean expression in $B B$ homozygote) $] / 2$ on a $\log _{2}$ scale. Positive value means $D$ high expression, and negative value means $B$ high expression. 
Adamts 4 and Igsf $4 b$ failed to show significant allelic expression difference. In the case of $U f_{c} 1$, the polarity of the allele effect failed to agree with the ASE result ( $D$ positive at $p=$ $0.02)$.

\subsubsection{High-Resolution Cis-QTL Mapping}

The BXD CNS data sets were generated from a combined panel of conventional RI strains and advanced RI strains that were derived by inbreeding advanced intercross progeny. The advanced RIs have approximately twice as many recombinations compared to standard RIs and the merged panel offers over a 3-fold increase in mapping resolution (Peirce et al., 2004). This expanded RI set combined with the relatively high intrinsic recombination rate within Qrrl (Paigen et al., 2008) provides comparatively high mapping resolution. Mapping precision can be empirically determined by analyzing cisQTLs in multiple large data sets, particularly the BXD Hippocampus Consortium, UMUTAffy Hippocampus, and Hamilton Eye data sets. These three data sets were selected because they have expression measurements from six BXD strains with recombinations in Qrr1. These strains-BXD8, BXD29, BXD62, BXD64, BXD68, and BXD84 - collectively provide six sets of informative markers and divide Qrrl into six non-recombinant segments, labeled as segments 1-6 (haplotype structures shown in Figure 5-2).

As cis-acting regulatory elements are usually located within a few kilobases of a gene's coding sequence (Cowles et al., 2002), we used the cis-QTLs as an internal metric of mapping precision by measuring the offset distance between a cis-QTL (position of peak QTL marker) and the parent gene (Figure 5-3). For cis-QTLs with LOD scores between 3-4 (genome-wide $p$ of 0.1-0.01) the mean gene-to-QTL peak distance is 900 $\mathrm{kb}$. The offset decreases to a mean of $640 \mathrm{~kb}$ for $c i s$-QTLs with LOD scores greater than $4(p<0.001)$. Very strong cis-QTLs with LOD scores greater than $11\left(p<10^{-6}\right)$ have a mean gene-to-QTL peak distance of only $450 \mathrm{~kb}$. In all, $60 \%$ of cis-QTLs we examined have peak linkage on markers located precisely in the same non-recombinant segment as the parent gene, and $30 \%$ have peak linkage on markers in a segment adjacent to the parent gene (dataset S1). These cis-QTLs provide an empirical metric of mapping precision within Qrrl.

Parsing trans-QTLs by high-resolution mapping and gene functions Mapping precision of cis-QTLs is comparatively higher in the BXD hippocampus data set (average offset of only $410 \mathrm{~kb}$ ), and we used this set to examine the trans-QTLs $(\mathrm{LOD} \geq 3$ ) at higher resolution. The trans-QTLs in $Q r r 1$ were parsed into subgroups based on the location of peak LOD score markers. This method of resolving trans-QTLs effectively grouped subsets of transcripts into functionally related cohorts. For instance, all the QTLs for the aminoacyl-tRNA synthetases (ARS) have peak LOD scores only within the distal three segments of Qrrl (Figure 5-4). This consistency in QTL peaks for transcripts of the same gene family is itself a good indicator of mapping precision. In addition to the ARS, numerous other genes involved in amino acid metabolism and translation map to the distal part of Qrr1 (e.g., Atf4, Asns, Eif4g2, and Pum2). 

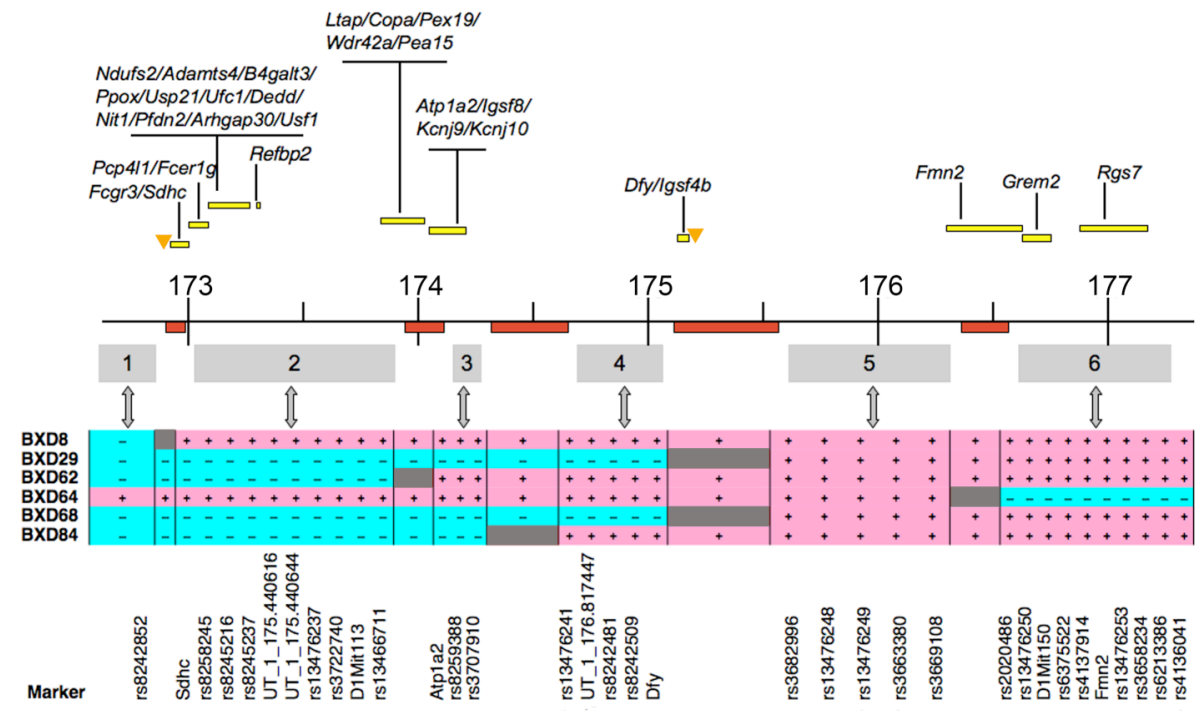

Figure 5-2 Haplotype maps of Qrr1 recombinant BXD strains

BXD8, BXD29, BXD62, BXD64, BXD68, and BXD84 have recombinations in Qrr1. B haplotype is assigned blue $(-), D$ haplotype is assigned pink $(+)$, and recombination regions are shown in grey. The Qrrl interval (in Mb scale) is shown above and approximate positions of recombination are highlighted (red). The recombinant strains collectively divide Qrr1 into six segments (labeled 1-6), and provide six sets of informative markers. Markers are shown below and approximate positions of candidate genes (yellow bars) and tRNA clusters (orange triangles) are indicated. 


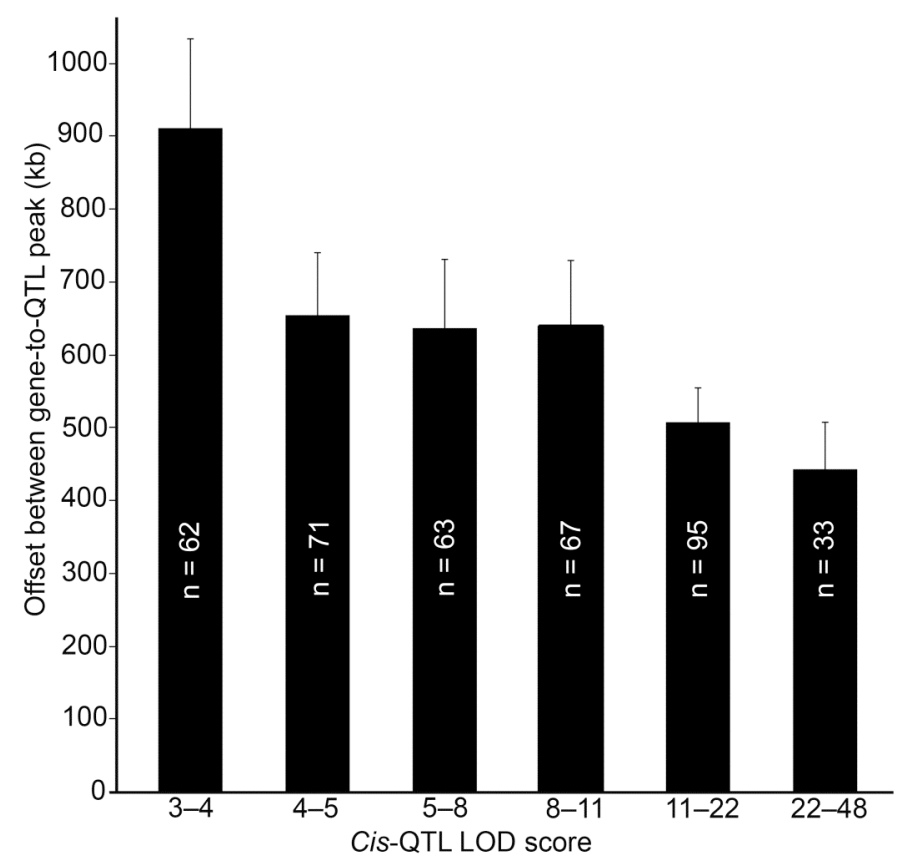

Figure 5-3 QTL mapping precision in Qrr1

Mapping precision was empirically determined by measuring the distance between a cisQTL peak and location of parent gene. Cis-QTLs in BXD Hippocampus Consortium, UMUTAffy Hippocampus, and Hamilton Eye data sets were used for this purpose. Mean gene-to-QTL peak distance (y-axis) was plotted as a function of LOD score (LOD score range on $\mathrm{X}$-axis). Number of probe sets in each LOD range is shown. Mapping precision increases with increase in LOD score. The mean offset for cis-QTLs with LOD scores 34 (genome-wide adjusted p-value of $0.1-0.01$ ) is $900 \mathrm{~kb}$, and the offset decreases to 650 $\mathrm{kb}$ at 4-5 LOD scores (p-value of 0.01-0.001). Cis-QTLs with LOD scores greater than 11 ( $\mathrm{p}$-value $<10^{-6}$ ) have mean offset of only $450 \mathrm{~kb}$. 


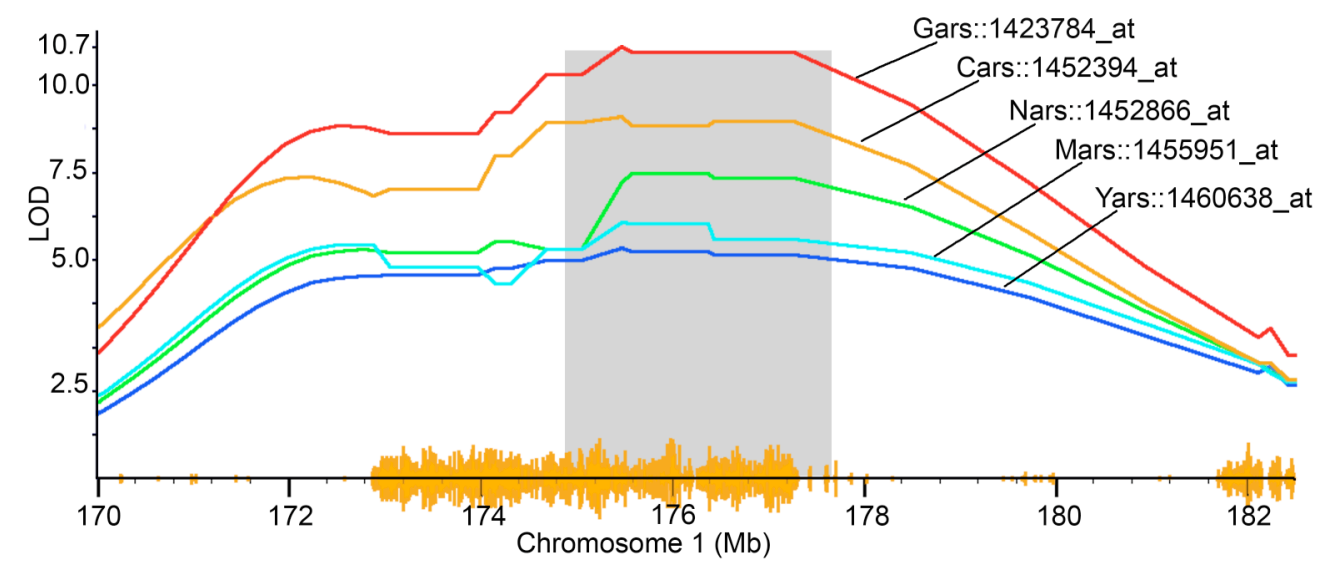

Figure 5-4 QTL for aminoacyl-tRNA synthetase in distal $\operatorname{Qrr1}$

Transcripts of Gars, Cars, Nars, Mars, and Yars map as trans-QTLs to Qrr1 at LOD > 4 (genome-wide $p<0.01$ ) in the BXD hippocampus data set. The trans-QTLs have peak LOD precisely on markers in distal part of Qrrl, $\sim 175-177.5 \mathrm{Mb}$ (shaded regions). Yellow seismograph on Chr 1 (x-axis) tracks SNP density between B and D alleles. Affymetrix probe set ID for each transcript is shown.

We divided the trans-QTLs into two broad subgroups - those with peak QTLs on markers in the proximal part of $\operatorname{Qrrl}(\mathrm{Qrrlp} ; 172-174.5 \mathrm{Mb}$ or segments 1, 2, 3 in Figure 5-2), and those with peak QTLs on markers in the distal part of Qrr1 (Qrrld; 174.5-177.5 $\mathrm{Mb}$ or segments 4, 5, and 6 in Figure 5-2). While Qrrlp is relatively gene-rich, only 35\% of the trans-QTLs (129 out of 365 probe sets) have peak LOD scores in this region. The majority of trans-QTLs - about 65\% (236 out of 365 probe sets) - have peak QTLs in the relatively gene-sparse $\operatorname{Qrrld}$.

The two subsets of transcripts - those with trans-QTLs in Qrrlp and those with trans-QTLs in Qrrld - were analyzed for overrepresented gene functions using the DAVID functional annotation tool (http://david.abcc.ncifcrf.gov/). This revealed distinct gene ontology (GO) categories enriched in the two subsets. Enriched GOs among the transcripts modulated by Qrrlp include GTPase-mediate signal transduction (modified Fisher's exact test $p=0.001)$, and structural constituents of ribosomes $(p=0.003)$. Transcripts modulated by Qrrld are highly enriched in genes involved in RNA metabolism $\left(p=4 \times 10^{-7}\right)$, tRNA aminoacylation $\left(p=1 \times 10^{-5}\right)$ and translation $(p=2 \times$ $\left.10^{-5}\right)$, RNA transport $(p=0.003)$, cell cycle $(p=0.004)$, and ubiquitin mediated protein catabolism $(p=0.006)$. Other GO categories show enrichment in both Qrrlp and Qrrld. For example, genes involved in RNA metabolism and ubiquitin-mediated protein catabolism are also overrepresented among the transcripts modulated by $\operatorname{Qrr} l p$ ( $p=$ 0.002 for RNA metabolism and $p=0.005$ for ubiquitin-protein ligases). This may either be due to limitations in QTL resolution, or due to multiple loci in Qrrlp and Qrrld controlling these subsets of transcripts. 


\subsubsection{An Aminoacyl-tRNA Synthetase Trans-QTL in Distal Qrr1}

A remarkable number of transcripts of the ARS gene family map to Qrrl. A total of 16 ARS transcripts have trans-QTLs at a minimum LOD score of 3 in one or multiple BXD, B6D2F2, and B6C3H CNS data sets (Table 5-4). In almost all cases, QTLs peak on markers on the distal part of Qrrl. Except for Hars, the B allele in Qrrl consistently increases expression by $10 \%$ to $30 \%$. In the case of Hars, the $D$ allele has the positive additive effect and increases expression by about $10 \%$.

We examined all probes or probe sets that target ARS and ARS-like genes in the B6xD2 CNS data sets. The Affymetrix platform measures the expression of 34 ARS and ARS-like genes; 24 of these map to Qrrl at LOD scores ranging from a low of 2 to a high of 12. Even in the case of the suggestive trans-QTLs (i.e., LOD values between 2 and 3), the $B$ allele in $\operatorname{Qrr} 1$ has the positive effect on expression. The ARS family is also highly represented among trans-QTLs in the B6C3HF2 brain data set. Thirty-seven probes in this data set target the tRNA synthetases, eleven of these have trans-QTLs in Qrrld (LOD scores ranging from 2 to 20), and almost all have a $B$ positive additive effect (exceptions are Hars and Qars). The co-localization of trans-QTLs to Qrrld, the general consensus in parental allele effect, and their common biological function indicate that there is a single QTL in the distal part of Qrrl modulating the expression of the ARS. It is crucial to note that this genetic modulation is only detected in CNS tissues.

In the LXS hippocampus data set, Qrr 1 has only a limited trans-effect on gene expression. Despite the weak effect, expression of Dars2 (probe ID ILM580427) maps to the distal part of $\operatorname{Qrr} 1$ at a LOD of 3. Although this is only a weak detection of the ARS QTL in the LXS data set, it nonetheless demonstrates the strong regulatory effect of Qrrl on the expression of this gene family. In the case of the CXB hippocampus data set, not a single trans-QTL for the ARS is detected in Qrrl.

\subsubsection{Trans-QTLs for Transcripts Localized in Neuronal Processes}

In addition to the high overrepresentation of transcripts involved in translation and RNA metabolism, several transcripts known to be transported to neuronal processes or involved in RNA transport also map to Qrrld, including Camk2a, Bdnf, Cdc42, Eif4e, Eif4g2, Hnrpab, Ppplcc, Pabpc1, Eif5, Kpnb1, Rhoip3, Stau2, and Pum2 (Duchaîne et al., 2002; Eberwine et al., 2002; Poon et al., 2006; Vessey et al., 2006). An interesting example is provided by the brain derived neurotrophic factor $(B d n f)$. Two alternative forms of $B d n f$ mRNA are known — one isoform has a long 3' UTR and is specifically transported into the dendrites; the other isoform has a short 3' UTR and remains primarily in the somatic cytosol (An et al., 2008) The Affymetrix M430 arrays contain two different probe sets that target these $B d n f$ isoforms. Probe set 1422169_a at targets the distal 3' UTR and is essentially specific for the dendritic isoform, and probe set 1422168_a_at targets a coding sequence common to both isoforms. Although both probe sets detect high expression signal in the hippocampus, only the dendritic isoform maps as a trans-QTL to Qrrld. This enrichment in transcripts that are transported to neuronal 
Table 5-4 Aminoacyl-tRNA synthetases that have trans-QTLs in Qrr1

\begin{tabular}{|c|c|c|c|c|c|c|}
\hline Gene & Name & ProbeID $^{A}$ & $\mathrm{Chr}^{\mathrm{B}}$ & Data set $^{\mathrm{C}}$ & LOD $^{\text {D }}$ & $\mathbf{B} / \mathbf{D}^{\mathrm{C}}$ \\
\hline Nars & $\begin{array}{l}\text { asparaginyl- } \\
\text { tRS }\end{array}$ & $\begin{array}{l}\text { 1452866_at } \\
\text { A }\end{array}$ & Chr 18 & $\begin{array}{l}\text { BXD } \\
\text { cerebellum }\end{array}$ & 12.0 & $B$ \\
\hline Gars & glycyl-tRS & 1423784_at & Chr 6 & $\begin{array}{l}\text { BXD } \\
\text { hippocampus }\end{array}$ & 10.6 & $B$ \\
\hline Rars & arginyl-tRS & 1416312_at & Chr 11 & $\begin{array}{l}\text { BXD } \\
\text { forebrain }\end{array}$ & 8.9 & $B$ \\
\hline Cars & cysteinyl-tRS & $\begin{array}{l}100244060 \\
01\end{array}$ & Chr 7 & $\begin{array}{l}\text { B6C3HF2 } \\
\text { brain }\end{array}$ & 8.9 & $B$ \\
\hline Yars & tyrosyl-tRS & $\begin{array}{l}100243998 \\
42\end{array}$ & Chr 4 & $\begin{array}{l}\text { B6C3HF2 } \\
\text { brain }\end{array}$ & 8.0 & $B$ \\
\hline Iars & isoleucine-tRS & $\begin{array}{l}1426705 \_s \\
\text { at }\end{array}$ & Chr 13 & $\begin{array}{l}\text { BXD } \\
\text { cerebellum }\end{array}$ & 7.8 & $B$ \\
\hline Sars & seryl-tRS & $\begin{array}{l}1426257 \_a \\
\text { at }\end{array}$ & Chr 3 & $\begin{array}{l}\text { BXD } \\
\text { cerebellum }\end{array}$ & 6.9 & $B$ \\
\hline Mars & $\begin{array}{l}\text { methionine- } \\
\text { tRS }\end{array}$ & 1455951_at & Chr 10 & $\begin{array}{l}\text { BXD } \\
\text { hippocampus }\end{array}$ & 6.5 & $B$ \\
\hline Hars & histidyl-tRS & $\begin{array}{l}\text { 1438510_a } \\
\text { at }\end{array}$ & Chr 18 & $\begin{array}{l}\text { BXD } \\
\text { hippocampus }\end{array}$ & 5.2 & $D$ \\
\hline Iars2 & isoleucine-tRS & 1426735_at & Chr 1 & $\begin{array}{l}\text { BXD } \\
\text { hippocampus }\end{array}$ & 4.3 & $B$ \\
\hline Tars & threonyl-tRS & $\begin{array}{l}100243956 \\
55\end{array}$ & Chr 15 & $\begin{array}{l}\text { B6C3HF2 } \\
\text { brain }\end{array}$ & 4.0 & $B$ \\
\hline Aars & alanyl-tRS & $\begin{array}{l}1451083 \_s \\
\text { at }\end{array}$ & Chr 8 & $\begin{array}{l}\text { BXD } \\
\text { eye }\end{array}$ & 3.9 & $B$ \\
\hline Lars & leucyl-tRS & $\begin{array}{l}1448403 \text { at } \\
\text { A }\end{array}$ & Chr 18 & $\begin{array}{l}\text { BXD } \\
\text { cerebellum }\end{array}$ & 3.7 & $B$ \\
\hline Ears2 & glutmyl-tRS & $\begin{array}{l}\text { ILM52904 } \\
46\end{array}$ & Chr 7 & $\begin{array}{l}\text { BXD ILM } \\
\text { striatum }\end{array}$ & 3.7 & $B$ \\
\hline $\begin{array}{l}\text { Aarsd } \\
1\end{array}$ & $\begin{array}{l}\text { alanyl-tRS } \\
\text { domain } 1\end{array}$ & 1424006_at & Chr 11 & $\begin{array}{l}\text { B6D2F2 } \\
\text { brain }\end{array}$ & 3.5 & $B$ \\
\hline Dars & aspartyl-tRS & $\begin{array}{l}\text { 1423800_at } \\
\text { A }\end{array}$ & Chr 1 & $\begin{array}{l}\text { BXD } \\
\text { cerebellum }\end{array}$ & 3.2 & $B$ \\
\hline
\end{tabular}


processes raises the possibility that this CNS specific trans-effect may be related to local protein synthesis.

\subsection{8 tRNAs in Qrrl}

Prompted by the many ARS transcripts that consistently map to Qrrld, we searched the genomic tRNA database (Lowe et al., 1997) for tRNAs in this region. Interestingly, distal Chr 1 is one of many tRNA hotspots in the mouse genome and several predicted tRNAs are clustered in the non-coding regions of Qrrl (Figure 5-2). The majority of these tRNA sequences are in the proximal end of Qrrl, over $2 \mathrm{Mb}$ away from Qrrld. We scanned the intergenic non-coding regions in Qrrld for tRNAs using the tRNAscan-SE software (Lowe et al., 1997) and uncovered tRNAs for arginine and serine, and three pseudo-tRNA sequences between genes Igsf4b and Aim2 (175.204-175.257

$\mathrm{Mb}$ ) in Qrrld (dataset S3). Transfer RNAs are involved in regulating transcription of the ARS in response to cellular amino acid levels (Rychelynck et al., 2005) and are functionally highly relevant candidates in Qrrld. Polymorphism in the tRNA clusters (e.g., possible copy number variants, differences in tRNA species) may have significant impact on the expression of the ARS.

\subsubsection{Sequence Analysis of Multiple Crosses}

Trans-regulation of large number of transcripts by Qrrl is a strong feature of crosses between B6 and D2 - both the BXD RI set and B6D2F2 intercrosses - and in the $\mathrm{B} 6$ and $\mathrm{C} 3 \mathrm{H}$ intercrosses. The feature is much weaker in the large LXS RI set and in the small CXB panel. The effect specificity demonstrates that a major source of the Qrrl signal is generated by variations between $B$ and $D$, and $B$ and $\mathrm{C} 3 \mathrm{H}$ alleles $(H)$ but not by variations between the ILS and ISS alleles ( $L$ and $S$, respectively), and $B$ and BALB alleles $(C)$. This contrast can be exploited to identify sub-regions that underlie the transQTLs (Hitzemann et al., 2002).

SNPs were counted for all four pairs of parental haplotypes $-B$ vs $D, B$ vs $H, B$ vs $C$, and $L$ vs $S$ - and SNP profiles for the four crosses were compared (Figure 5-5). Qrr 1 is a highly polymorphic interval in the $\mathrm{B} 6 \mathrm{xD} 2$ crosses. The flanking regions, however, have few SNPs (170-172.25 Mb proximally, and 177.5-179.5 Mb distally) and are almost identical-by-descent between B6 and D2. The B6xBALB crosses, despite being negative for the trans-effect, have moderate to high SNP counts in Qrrl and share a SNP profile somewhat similar to $\mathrm{B} 6 \mathrm{xD} 2$ crosses. The $\mathrm{B} 6 \mathrm{xC} 3 \mathrm{H}$ crosses also have moderate to high SNP counts in Qrrl, with a relatively higher SNP count in Qrrld compared to Qrr 1p. In contrast, in the LXS, Qrr 1p is more SNP-rich than Qrrld. Most notably, the segments that harbor the tRNAs and candidates Fmn2, Grem2, and Rgs 7 are almost identical by descent between ILS and ISS. This SNP comparison indicates that the strongest trans-effect is from Qrrld. A possible reason why the trans-effect is not detected in the CXB RI strains, despite being SNP rich in Qrr 1, is that the crucial SNPs underlying the trans-QTLs may not be segregating in this cross or that undetected copy 


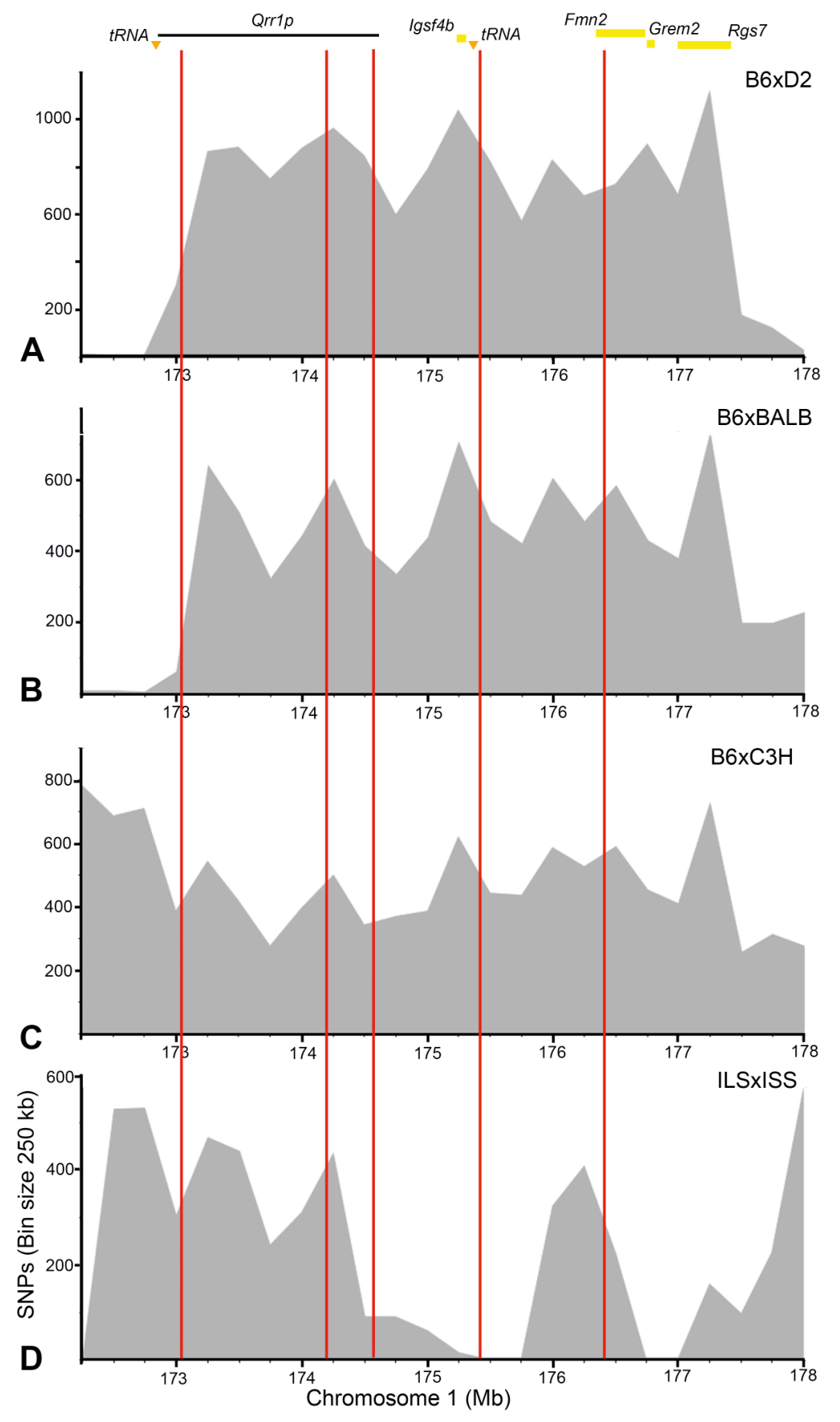

Figure 5-5 SNP comparison of multiple crosses

SNPs in Qrr1 were counted for (A) C57BL/6J (B6) x DBA/2J (D2), (B) B6 x BALB/cBy (BALB), (C) $\mathrm{B} 6 \times \mathrm{C} 3 \mathrm{H} / \mathrm{HeJ}(\mathrm{C} 3 \mathrm{H})$, and (D) ILS x ISS. Vertical red lines mark the approximate positions of recombination. Region covered by $\operatorname{Qrrlp}$ (horizontal line), candidate genes in Qrrld (yellow bars), and position of tRNA clusters (triangles) are shown above the graphs. The $\mathrm{B} 6 \mathrm{xD} 2, \mathrm{~B} 6 \mathrm{xBALB}$, and $\mathrm{B} 6 \mathrm{xC} 3 \mathrm{H}$ crosses have moderate to high SNP counts throughout $Q r r 1$. In the ILSxISS cross, Qrrlp is relatively SNP-rich but Qrrld is SNP-sparse. 
number variants make important contributions to the Qrrl effects. A final explanation may be that the small CXB data set (13 strains) is simply underpowered.

\subsubsection{High-Ranking Candidates Based on Cross-Specificity of Cis-QTLs}

We used the specificity of cis-QTLs in the multiple crosses to identify higher priority candidates in $\mathrm{Qrrl}$. The assumption is that candidate genes whose transcripts have cis-QTLs (LOD score above 3) in the $\mathrm{B} 6 \mathrm{xD} 2$ and $\mathrm{B} 6 \mathrm{xC} 3 \mathrm{H}$ crosses but not in the LXS and CXB RI strains are stronger candidates for trans-QTLs that are detected in the former two crosses but not in the latter two crosses. In contrast, cis-QTLs with the inverse cross specificity are less likely to underlie these trans-QTLs. Based on this criterion, there are four high-ranking candidates in Qrrlp-Purkinje cell protein 4-like 1 $(P c p 4 l 1)$, prefoldin $(P f d n 2)$, WD repeat domain 42 a (Wdr42a), and Kcnj10 (Table 5-2). There are only two high-ranking candidates in Qrrld-formin 2 (Fmn2), an actin binding protein involved in cytoskeletal organization, and regulator of G-protein signaling 7 (Rgs7) (Table 5-2).

Both Fmn2 and Rgs 7 are almost exclusively expressed in the CNS and are high priority candidates for the CNS specific trans-QTLs. A point of distinction between the two candidates is that while expression of $R g s 7$ maps as a cis-QTL only in the B6xD2 and $\mathrm{B} 6 \mathrm{xC} 3 \mathrm{H}$ crosses, expression of Fmn2 maps as a cis-QTL in $\mathrm{B} 6 \mathrm{xD} 2$ and $\mathrm{B} 6 \mathrm{xC} 3 \mathrm{H}$ crosses, and in the CXB RI strains in which the trans-effect is not detected (Table 5-2). Based on the pattern of specificity of cis-QTLs in multiple crosses, $\operatorname{Rgs} 7$ is a more appealing candidate. However, Fmn2 has known missense SNPs that segregate in the B6xD2 (Glu610Asp, Pro1077Leu, Asp1431Glu) and B6xC3H crosses (Val372Ala). There are no known missense mutations in Fmn2 in the CXB and LXS RI strains, and no known missense mutation in $\operatorname{Rgs} 7$ in any of the four crosses.

\subsubsection{Partial Correlation Analysis}

Linkage disequilibrium (LD) is a major confounding factor that limits fine-scale discrimination among physically linked candidates in a QTL. To further evaluate the two high-priority candidates in Qrrld-Fmn2 and $\mathrm{Rgs} 7$ - we implemented a partial correlation analysis (de La Fuente et al., 2004) in which the effect of genotype at Qrrld was controlled. For this analysis, we computed the partial correlation coefficient between cis-regulated transcripts and each trans-regulated transcript after regression against the Qrrld genotype. This partial correlation reveals residual variance that links cis candidates with trans targets, independent of genetic variance at Qrrld. We computed the partial correlation between $R g s 7$ and Fmn2, and 14 transcripts representative of the different GOs that map to Qrrld. The highest partial correlations are between Fmn2 and Rnf6 ( $r=$

$\left.0.68, p<10^{-13}\right)$, Atf4 $\left(r=0.6, p<10^{-9}\right)$, Asns $\left(r=0.55, p<10^{-7}\right)$, Ube2d3 $(r=0.5, p<$ $\left.10^{-6}\right)$, Hnrpk $\left(r=0.5, p=10^{-5}\right)$, Rab2 $\left(r=-0.5, p=10^{-5}\right)$, and Gars $\left(r=0.5, p=10^{-5}\right)$. The strongest correlate of $F m n 2$ is $R n f 6$, a gene involved in regulating actin dynamics in 
axonal growth cones (Tursun et al., 2005) Although not unequivocal, this analysis provides stronger support for Fmn2 than for Rgs 7 .

\subsubsection{Effect of Fmn2-Deletion on Gene Expression}

Fmn2 is almost exclusively expressed in the nervous system (Leader et al., 2000) and is a strong candidate for a trans-effect specific to neural tissues. However, its precise function in the brain has not been established. Fmn2-null mice do not have notable CNS abnormalities (Leader et al., 2002), but to evaluate a possible role of $F m n 2$ on expression of genes that map to Qrrld, we generated array data from brains of $F m n 2$-null $\left(F m n 2^{-/}\right)$ and coisogenic $\left(\mathrm{Fmn}^{+/+}\right)$129/SvEv controls. At a stringent statistical threshold (Bonferroni corrected $p<0.05$ ), only eight genes have significant expression differences between $\mathrm{Fmn}_{2} \mathrm{~F}^{-/}$and $\mathrm{Fmn} 2^{+/+}$genotypes (Table 5-5). Five out of the eight genes, including Pou6f1, Usp53, and Slc1 la, have trans-QTLs in Qrrld. Deletion of Fmn2 had the most drastic effect on the expression of the transcription factor gene Pou6fl, a gene implicated in CNS development and regulation of brain-specific gene expression (Cui et al., 1998; Drews et al., 2007). Expression of Pou6f1 maps as a trans-QTL (at LOD score of 3) to Qrrld in the hippocampus data set, and its expression was down-regulated more than 44-fold in the Fmn2 $2^{-/-}$line. While the expression analysis of Fmn2-null mice does not definitively link all the trans-QTLs to Fmn2, variation in this gene is likely to underlie some of the trans-QTLs in Qrrld. The possible compensatory mechanism in the Fmn2-null CNS, and the different genetic background of the mice (129/SvEv) are factors that may have contributed to the weak detection of trans-effects in the knockout line.

\subsubsection{Sub-Cellular Localization of FMN2 in Hippocampal Neurons}

We examined the intracellular distribution of FMN2 protein in neurons using immunocytochemical techniques. All hippocampal pyramidal neurons on a culture dish exhibited distinct and fine granular immunoreactivity for FMN2. The cell body itself had the strongest signal (Figure 5-6). This fine punctate labeling extended into proximal dendrites and could be followed into distal dendrites. In some instances very thin processes, possibly the axons, were also labeled.

\subsubsection{Linking Expression and Classical QTLs: Szs1}

The strong trans-effect that Qrrl has on gene expression is a likely basis for the classical QTLs that map to this region. For example, the major seizure susceptibility QTL (Szs1) has been precisely narrowed to Qrrlp (Ferraro et al., 2007). We found that 10 genes already known to be associated with seizure or epilepsy have trans-QTLs with peak LOD scores near Szsland in Qrrlp. These include Scn1b, Cacnalg, Pnpo, and Dapkl (Table 5-6). In every case, the $D$ allele has the positive additive effect on the expression of these seizure related transcripts, increasing expression $5 \%$ to $20 \%$. The two potassium channel genes, Kcnj9 and Kcnj10, are the primary candidates (Ferraro et al., 
Table 5-5 Genes differentially expressed between Fmn2 $2^{+/+}$and Fmn2

\begin{tabular}{|c|c|c|c|c|c|c|c|}
\hline Gene & $\mathrm{Chr}^{\mathrm{A}}$ & $\begin{array}{c}\boldsymbol{F m n} \\
2^{+/+\mathrm{B}} \\
\end{array}$ & $\begin{array}{c}F m n \\
2^{-/-\mathrm{B}} \\
\end{array}$ & Fold $^{C}$ & $P^{\mathbf{D}}$ & $\mathbf{L O D}^{\mathbf{E}}$ & Dataset $^{\mathrm{E}}$ \\
\hline Pou6f1 & 15 & 11.96 & 6.48 & 45 & $\underset{-6}{3 X} 10$ & 3.0 & $\begin{array}{l}\text { BXD } \\
\text { Hippocampus }\end{array}$ \\
\hline Zfp 420 & 7 & 10.12 & 7.70 & 5 & 0.002 & & \\
\hline Txnll & 18 & 10.72 & 6.70 & 16 & 0.002 & 3.0 & $\begin{array}{l}\text { B6D2F2 } \\
\text { striatum }\end{array}$ \\
\hline Usp53 & 3 & 7.17 & 9.32 & 4 & 0.009 & 3.3 & $\begin{array}{l}\text { BXD } \\
\text { Hippocampus }\end{array}$ \\
\hline $\begin{array}{l}\text { LOC } 33113 \\
9\end{array}$ & 4 & 14.45 & 10.59 & 15 & 0.01 & & \\
\hline Slcl1a2 & 15 & 9.92 & 9.17 & 2 & 0.02 & 3.9 & $\begin{array}{l}\text { BXD } \\
\text { Hippocampus }\end{array}$ \\
\hline Pgbd5 & 8 & 13.40 & 12.12 & 2 & 0.02 & 3.3 & $\begin{array}{l}\text { BXD HBP } \\
\text { Striatum }\end{array}$ \\
\hline $\begin{array}{l}\text { 6330569M2 } \\
\text { 2Rik }\end{array}$ & 3 & 6.42 & 10.63 & 18 & 0.03 & & \\
\hline
\end{tabular}

${ }^{\text {A }}$ Physical location of gene; ${ }^{\mathbf{B}}$ Average expression signal in Fmn2-null and wild-type lines;

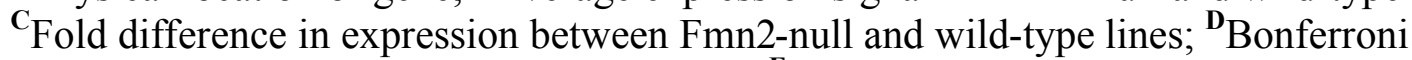
adjusted $p$-values; corrected for 46,620 tests; ${ }^{\mathbf{E}}$ Highest LOD in Qrrl and data set in which transcript has highest LOD in Qrrl. 


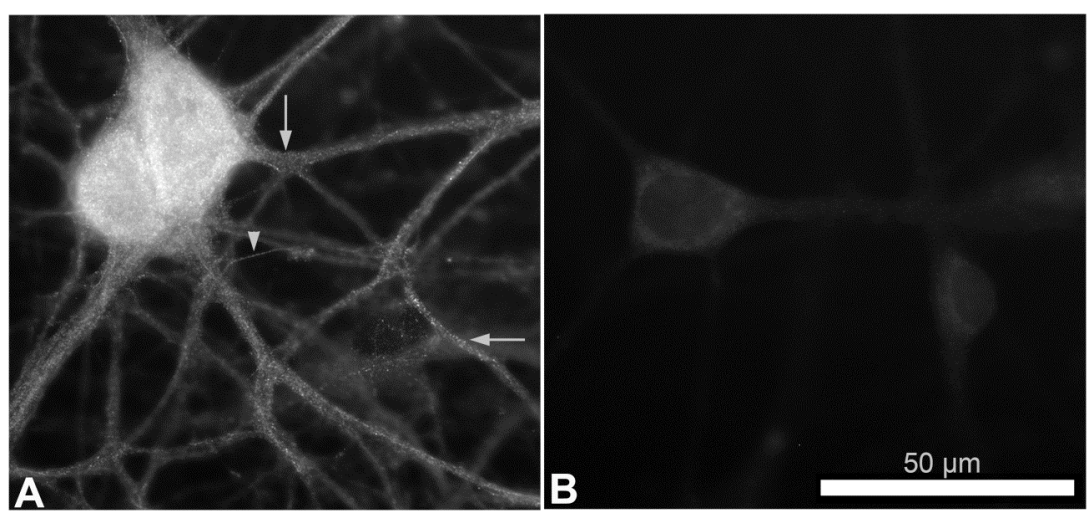

Figure 5-6 Expression of FMN2 protein in hippocampal neurons

(A) Neurons exhibited pronounced fine granular immunoreactivity for FMN2. The cell body had the strongest signal. The fine granular staining extended into apical and distal dendrites (arrows). Thin axon-like processes were also labeled (arrow head). (B) The fine granular staining is not detected in controls of sister cultures processed in parallel without the first antibody. 
Table 5-6 Seizure associated genes with trans-QTLs in Qrr1p

\begin{tabular}{llllll}
\hline \multicolumn{1}{c}{ Gene } & Chr $^{\mathbf{A}}$ & \multicolumn{1}{c}{ Data set $^{\mathbf{B}}$} & LOD $^{\mathbf{C}}$ & B/D $^{\mathbf{D}}$ & \multicolumn{1}{c}{ Reference } \\
Scn1b & Chr 7 & Forebrain & 3.5 & & \\
Akt1 & Chr 12 & Forebrain & 3.0 & $\mathrm{D}$ & Wallace et al., 1998 \\
Cacnalg & Chr 11 & Forebrain & 2.8 & D & Sonshall et al., 2002 \\
Pnpo & Chr 11 & Cerebellum & 29 & $\mathrm{D}$ & Mills et al., 2004 \\
Dapk1 & Chr 13 & Cerebellum & 6.3 & $\mathrm{D}$ & Henshall et al., 2004 \\
Kcnma1 & Chr 14 & Cerebellum & 3.7 & $\mathrm{D}$ & Du et al., 2005 \\
Socs2 & Chr 10 & Cerebellum & 3.7 & $\mathrm{D}$ & Rosell et al., 2003 \\
Nsf & Chr 11 & Cerebellum & 3.3 & $\mathrm{D}$ & Yin et al., 2005 \\
Grm2 & Chr 9 & Cerebellum & 3.3 & $\mathrm{D}$ & Thomsen et al., 1994 \\
Adora1 & Chr 1 & Hippocampus & 3.0 & D & Fedele et al., 2006 \\
\hline
\end{tabular}

${ }^{\mathrm{A}}$ Physical location of gene; Adoral is located on Chr 1 at $136 \mathrm{Mb} ;{ }^{\mathrm{B}}$ Dataset in which transcript has highest trans-QTL in Qrrlp; ${ }^{\mathbf{C}}$ Highest LOD scores in Qrrlp; ${ }^{\mathbf{D}}$ Allele that increases expression.

2007). Both are strongly cis-regulated. The tight linkage between these genes (within 100 $\mathrm{kb}$ ) limits further genetic dissection, but in situ expression data from the Allen Brain Atlas (ABA, http://www.brain-map.org) provides us with a powerful complementary approach to evaluate these candidates (Lau et al., 2008). Kcnj9 (Figure 5-7A) is expressed most heavily in neurons within the dentate gyrus, whereas Kcnj10 (Figure 5-7B) is expressed diffusely in glial cells in all parts of the CNS. The seizure-related transcripts with trans-QTLs near Szs 1 are most highly expressed in neurons, and all have comparatively high expression in the hippocampus. Furthermore, expression patterns of six of the seizure transcripts that map to Qrrlp show spatial correlations with Kcnj9.

Dapkl and Cacnalg (Figure 5-7C) have expression pattern that match Kcnj9 with strong labeling in the dentate gyrus and CA1, and weaker labeling in CA2 and CA3. In contrast, Socs2 (Figure 5-7D), Adoral, Pnpo, and Kcnmal complement the expression of Kcnj9 with comparatively strong expression in CA2 and CA3, and weak expression in CA1 and dentate gyrus.

\subsection{Discussion}

Qrrl is a complex regulatory region that modulates expression of many genes and classical phenotypes. By exploiting a variety of microarray data sets and by applying a combination of high-resolution mapping, sequence analysis, and multiple cross analysis, we have dissected Qrrl into segments that are primarily responsible for variation in the expression of functionally coherent sets of transcripts. The distal portion of $\mathrm{Qrrl}$ ( $\mathrm{Qrrld}$ ) has a strong trans-effect on RNA metabolism, translation, tRNA aminoacylation, and transcripts that are transported into neuronal dendrites. Fmn2, Rgs 7, and a cluster of tRNAs are strong candidates in Qrrld. We analyzed gene expression changes in the CNS of Fmn2-null mice and detected a profound effect on the expression of a small number of 


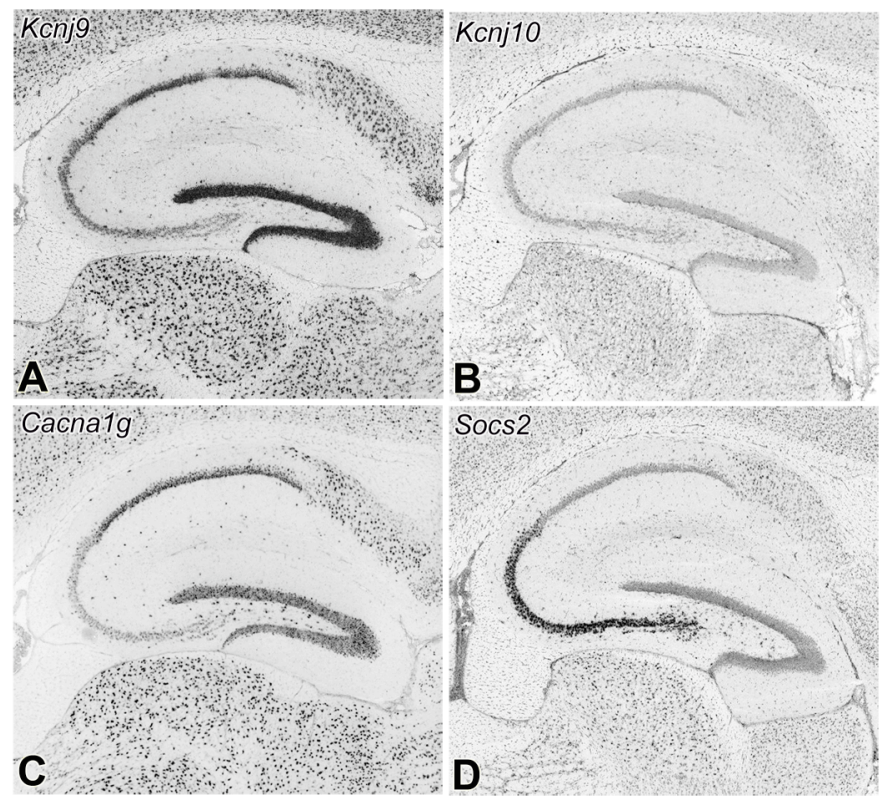

Figure 5-7 Expression pattern of seizure-related genes with QTLs in Qrr1 Candidate gene Kcnj9 (A) has heavy expression in neurons. Kcnj9 shows a regionally restricted expression in the hippocampus with intense labeling in dentate gyrus, strong labeling in CA1, and relatively weak labeling in CA2 and CA3. Candidate gene Kcnj10 (B) has a more diffused pattern and expressed primarily in glial cells. There is almost no labeling for Kcnj10 in the hippocampus. Transcripts of seizure-related genes, Cacnalg (C) and Socs2 (D), have trans-QTLs in Qrrlp. Both genes show high expression in neurons. Cacnalg matches the expression of Kcnj9 with strong labeling in dentate gyrus and CA1, and weak labeling in CA2 and CA3. Socs 2 complements the expression of Kcnj9 and Cacnalg with intense labeling in CA2 and CA3. In situ expression data are from the Allen Brain Atlas. 
transcripts that map to Qrrld, particularly on the expression of the transcription factor Pou6f1. We have shown that the FMN2 protein is highly expressed in the cell body and processes of neurons, and is a high priority candidate in Qrrld.

\subsubsection{Kcnj9 vs. Kncj10 and Seizure Susceptibility}

The two inwardly rectifying potassium channel genes - Kcnj9 and Kcnj10 - are strong candidates for the seizure susceptibility QTL in Qrrlp that has been unambiguously narrowed to the short interval from Atpla 2 to Kcnj10 (Ferraro et al., 2004). In BXD CNS data sets, Qrr1 also modulates the expression of a set of genes implicated in the etiology of seizure and epilepsy, including Pnpo, Scn1b, Kcnmal, Socs2, and Cacnalg. Polymorphisms in the Kcnj9/Kcnj10 interval that influence expression of these genes are excellent candidates for the Szsl locus.

The in situ expression data in the ABA shows a striking spatial correlation between expression of Kcnj9 and other seizure-related transcripts that have trans-QTLs in $Q r r 1 p$. The complementary expression of Kcnj9 and the seizure-related transcripts make Kcnj9 a stronger candidate than Kcnj10. Kcnj9 has over a 2-fold higher expression in D2 (Hitzemann et al., 2003), a seizure prone strain, compared to B6, a relatively seizure resistant strain, suggesting that the proximal cause of Szs 1 may be high expression of this gene, perhaps due to the promoter polymorphism discovered by Hitzemann and colleagues (2003).

\subsubsection{Multiple Loci in a Major QTL Interval}

Fine mapping of complex traits have often yielded multiple constituent loci within a QTL interval (Legare et al., 2000; Demarest et al., 2001). Our mapping analyses of expression traits also show that multiple gene variants, rather than one master regulatory gene, cause the aggregation of expression QTLs in Qrrl. Subgroups of genes with tight coexpression can be dissected from the dense cluster of QTLs. Most notable is the strong trans-regulatory effect of Qrrld on genes involved in amino acid metabolism and translation, including a host of ARS transcripts. However, there are limits to our ability to dissect $Q r r 1$, and genes associated with protein degradation and RNA metabolism map throughout the region. In part this may be due to inadequate mapping resolution, but it may also reflect clusters of functionally related loci and genes (Petkov et al., 2006). At this stage we are also unable to discern whether there is a single or multiple QTLs within Qrrld. While it is likely that a single QTL modulates the expression of the ARS, there may be additional gene variants in Qrrld that modulate other transcripts involved in translation and RNA metabolism. With increased resolving power it may be possible to further subdivide transcripts that map to Qrrlp and Qrrld into smaller functional modules.

There may be multiple loci in Qrrl that modulate different stages of protein metabolism in the CNS. Maintenance of cellular protein homeostasis requires finely 
tuned cross talk between transcription and RNA processing, the translation machinery, and protein degradation (Ding et al., 2007), gene functions highly overrepresented among the transcripts that map to Qrrl. While these are generic cellular processes, there are unique demands on protein metabolism in the nervous system. Neurons are highly polarized cells and specialized mechanisms are in place to manage local protein synthesis and degradation in dendrites and axons (Steward et al., 2003). The nervous system is also particularly sensitive to imbalances in protein homeostasis (Malgaroli et al., 2006), a possible reason why the trans-effects of Qrrl are detected only in neural tissues.

\subsubsection{Candidates in Qrrld and Possible Link with Local Protein Synthesis}

Transfer RNAs are direct biological partners of the ARS, and the cluster of tRNAs in the highly polymorphic intergenic region of Qrrld is an enticing candidate. In addition to their role in shuttling amino acids, tRNAs also act as sensors of cellular amino acid levels and regulate transcription of genes involved in amino acid metabolism and the ARS (Ryckelynck et al., 2005). There is tissue specificity in the expression of different tRNA isoforms (Dittmar et al., 2006) and we speculate that the tRNA cluster in Qrrld is specifically functional in neural tissues.

$\operatorname{Rgs} 7$, a member of the RGS (regulator of G-protein signaling) family, is another high-ranking candidate in Qrrld. RGS proteins are important regulators of G-protein mediated signal transduction. $\operatorname{Rgs} 7$ is predominantly expressed in the brain and has been implicated in regulation of neuronal excitability and synaptic transmission (Saitoh et al., 1999; Hunt et al., 2003). Although RGS proteins are usually localized in the plasma membrane, RGS7 has been found to shuttle between the membrane and the nucleus (Drenan et al., 2005). This implies a role for RGS7 in gene expression regulation in response to external stimuli.

Our final high-ranking candidate in Qrrld is Fmn2. It codes for an actin binding protein exclusively expressed in the CNS and oocytes, and is involved in the establishment of cell polarity (Leader et al., 2000; Leader et al., 2002). In Drosophila, the formin homolog, cappuccino, has a role in RNA transport and in localizing the staufen protein to oocyte poles (Emmons et al., 1995; Johnstone et al., 2001). It is possible that FMN2 has parallel functions in mammalian neurons. Interestingly, Staufen 2 (Stau2), a gene involved in RNA transport to dendrites (Duchaîne et al., 2002), maps to Qrrld in BXD CNS data sets. Furthermore, deletion of formin homologs in yeast results in inhibition of protein translation (Gross \& Kinzy, 2007), compelling evidence for an interaction between the protein translation system and formins. Evidence for a role for Fmn2 in dendrites also comes from our immunocytochemical analysis that clearly demonstrates the expression of FMN2 protein in dendrites. Taken together, Fmn2 is a functionally relevant candidate gene in Qrrld and may be related to RNA transport and protein synthesis in the CNS. 


\section{CHAPTER 6. SUMMARY AND CONCLUDING REMARKS}

The work presented in this dissertation is an integrative, systems genetics approach to explore the molecular factors and brain structures that underlie emotional processing and behavior. The overarching question that was pursued in this study was how does information in the DNA sequence give rise to the rich variants of neural and behavioral traits? A combination of analytical methods, including morphometric analysis, classic QTL mapping, behavioral assays, gene expression analysis, and expression QTL mapping was used to identify the genetic modulators of the corticolimbic brain structures, particularly the basolateral amygdala (BLA), and associated emotion-related behavior.

The molecular origins of psychological conditions such as stress, anxiety and fear, and the resultant behavioral traits are extraordinarily complex and arise from the interaction of a host of genetic, epigenetic, and environmental factors. The integrative approach taken by system genetics is to consider each phenotype within the context of a vast network of causal factors and interrelated phenotypes. For example, multiple gene variants and molecular traits define the cellular and physiological traits that make up neural structures such as the BLA, which in turn give rise to higher-order cognitive and behavioral traits. In this light, genetic variations can be viewed as perturbations that resonate across multiple phenotypic levels.

Within the framework of systems genetics, each phenotype that was examined in this study was considered in relation to other phenotypes. The analytical strategy can be broken down into four basic steps: (1) exploit natural variations in neuroanatomy to uncover genes that may have pleiotropic influence on brain structure and behavior, (2) examine the association between variations in neuroanatomy and behavior, (3) examine gene expression (both baseline and in response to stressful experience) in the neuroanatomic substrates of behavior, and (4) examine genetic regulators of expression traits to identify genes and gene-networks that underlie higher-order structural and behavioral traits.

In the first step, variation in the size and cytoarchitecture of the BLA was analyzed to identify the underlying DNA sequence variants. As a central modulator of emotional processes, we reasoned that such variations associated with the BLA might be coupled to variations in fear, anxiety and stress responses. This analysis was done in a genetically segregating population of BXD RI mouse strains and harvests the power of natural variations to uncover multiple genes and gene-networks. This stands in contrast to the very reductive approach of examining a single or few genes at a time, as is the case with most gene knockout and transgenic studies. Genetic mapping of the BLA traits uncovered multiple QTLs linked to the BLA neuroanatomy (Vol8a in Chr 8, and loci on Chrs 11 and 13). This study implicated genes with no prior known association with the BLA (e.g., Large, Neurocan), and also genes already known to be involved in the development of the BLA, e.g., NeuroD2 (Lin et al., 2005). 
Following the morphometric and QTL analyses, the second step was to examine whether the variation in BLA neuroanatomy could indeed predict behavioral outcome. A correlative analysis with a variety of behavioral traits collected from the BXD strains showed significant correlations between the BLA and emotion- and addiction-related behavior. A systematic analysis was also done to compare the behaviors of BXD strains with small, medium, and large BLAs. The three groups of BXD strains showed significant differences in fear learning and stress reactivity. Mice with smaller BLA volumes showed significantly higher fear responses to conditioned auditory stimuli and contextual stimuli. The smaller BLA group also showed a more pronounced glucocorticoid response to stress. Given that the neuroanatomical data and behavioral data were collected from two different sites (UTHSC and NIH, respectively), this association between the traits demonstrates the robustness of the effect of BLA size on emotion-related processes.

To introduce more genetic diversity, we then went on to survey a panel of seven inbred strains (129/SvImJ, A/J, BALB/cJ, BALB/cByJ, FVBN/J, and B6 and D2) for emotion-related behavior. We also asked how these strains differed in their relative vulnerability to stressful experience by examining their behavior prior to, and following stress. We found that B6 and D2 showed the most interesting divergence in behavior. Consistent with previous studies, D2 showed a higher anxiety-like trait compared to B6. In addition, while B6 remained relatively resilient to restraint-stress (i.e., this strain showed no difference in anxiety-like behavior before and after restraint), D2 mice showed heightened anxiety-like behavior after restraint-stress.

In the third step, to elucidate the molecular correlates of such behavioral contrasts, we examined gene expression in the amygdala (primarily the BLA) of B6 and D2 mice. The prefrontal cortex and hippocampus were also included in the expression analysis as these two regions have also been implicated in stress-responsiveness and anxiety-related behavior. By comparing both baseline expression between B6 and D2, and expression changes following restraint-stress, we hoped to identify genes that contribute to the striking trait divergence that characterizes these two strains. This study revealed substantial quantitative differences in expression between B6 and D2. There was also marked strain difference in the set of genes that showed stress-induced changes in expression. Interestingly, several of the genes that were identified by this study have been implicated in human neuropsychiatric disorders (e.g. Rgs2, $\mathrm{Nr} 4 \mathrm{a} 2$, Comt, and clock genes such as Dbp, Per1, Per2, and Per3).

In the fourth step, we sought to identify DNA sequence variants that influence both molecular phenotypes as well as phenotypes at the structural and behavioral level. A remarkable region located on the distal end of mouse Chr $1(172-178 \mathrm{Mb})$ was the ideal subject for such an integrative study. This region, which we have named as Qrrl (QTL rich region on distal Chr 1), is known for its unusually high density of QTLs for neural and behavioral traits, e.g., traits like anxiety-related behavior, seizure, hippocampal volume, and alcohol preference consistently map to this region. Additionally, Qrrl is also an expression QTL hotspot and controls the expression of hundreds of mRNA transcripts in neural tissues. Our analysis of Qrrl uncovered much complexity in this QTL region. 
Rather than one "master-regulator" gene controlling multiple phenotypes, Qrrl appears to be a complicated cluster of QTLs controlling modules of gene-networks. At the current level of resolution, we were able to delineate a proximal region ( $Q r r l p)$ in which Kcnj9 is a strong candidate, and a distal region $($ Qrrld $)$ in which Fmn2 is the strongest candidate. Increasing the mapping resolution may be able to further subdivide the proximal and distal regions into even finer regulatory loci. This influence on gene expression may be the reason why so many classical QTLs have been mapped to Qrrl.

The complexity highlighted by Qrr1 may very well be the rule rather than the exception for loci that modulate complex traits. Efforts to fine-map a single QTL have often been confronted by clusters of multiple small effect QTLs within the original interval (Legare et al., 2000; Demarest et al., 2001). This poses a serious challenge, and to date, only a small handful of genes have been definitively identified for complex traits. Our own efforts to identify a causal gene were stymied by the compound nature of QTLs and the high gene density in Qrrl, and in Vol8a. Furthermore, it is now becoming clear that in addition to the canonical candidate genes, there are multiple spliced variants, microRNAs, and epigenetic factors to be considered.

With what appears to be an increasingly complex genomic landscape, it is now all the more necessary to apply the multipronged approach taken by systems genetics. The work expounded in this dissertation is an example of such an integrative approach and could be considered as the groundwork for even deeper analyses of the information coded in the DNA. Further advancement in genomic technology, higher resolution sequencing of the DNA, and more sophisticated statistical and analytical techniques will ultimately yield answers that, up to this point, may seem elusive. 


\section{LIST OF REFERENCES}

Abarca C, Albrecht U, Spanagel R (2002). Cocaine sensitization and reward are under the influence of circadian genes and rhythm. Proc Natl Acad Sci U S A 99:9026-9030.

Abell F, Krams M, Ashburner J, Passingham R, Friston K, Frackowiak R et al. (1999).

The neuroanatomy of autism: a voxel-based whole brain analysis of structural scans. Neuroreport 10:1647-1651.

Airey DC, Lu L, Shou S, Williams RW (2002). Genetic sources of individual differences in the cerebellum. Cerebellum 1:233-240.

Airey DC, Lu L, Williams RW (2001). Genetic control of the mouse cerebellum: identification of quantitative trait loci modulating size and architecture. J Neurosci 21:5099-5109.

Airey DC, Robbins AI, Enzinger KM, Wu F, Collins CE (2005). Variation in the cortical area map of $\mathrm{C} 57 \mathrm{BL} / 6 \mathrm{~J}$ and $\mathrm{DBA} / 2 \mathrm{~J}$ inbred mice predicts strain identity. BMC Neurosci $6: 18$.

Amat J, Baratta MV, Paul E, Bland ST, Watkins LR, Maier SF (2005). Medial prefrontal cortex determines how stressor controllability affects behavior and dorsal raphe nucleus. Nat Neurosci 8:365-371.

American Psychiatric Association 1994. Diagnostic and Statistical Manual of Mental Disorders IV. American Psychiatric Press, Washington, DC.

An JJ, Gharami K, Liao GY, Woo NH, Lau AG et al. (2008). Distinct role of long 3' UTR BDNF mRNA in spine morphology and synaptic plasticity in hippocampal neurons. Cell 134:175-187.

Anglada-Figueroa D, Quirk GJ (2005). Lesions of the basal amygdala block expression of conditioned fear but not extinction. J Neurosci 25:9680-9685.

Anisman H, Hayley S, Kelly O, Borowski T, Merali Z (2001). Psychogenic, neurogenic, and systemic stressor effects on plasma corticosterone and behavior: mouse straindependent outcomes. Behav Neurosci 115:443-454.

Anisman H, Matheson K (2005). Stress, depression, and anhedonia: caveats concerning animal models. Neurosci Biobehav Rev 29:525-546.

Aronsson M, Fuxe K, Dong Y, Agnati LF, Okret S, Gustafsson JA (1988). Localization of glucocorticoid receptor mRNA in the male rat brain by in situ hybridization. Proc Natl Acad Sci USA 85:9331-9335.

Bannerman DM, Rawlins JN, Good MA (2006). The drugs don't work-or do they? Pharmacological and transgenic studies of the contribution of NMDA and GluR-Acontaining AMPA receptors to hippocampal-dependent memory. Psychopharmacology (Berl) 188:552-566.

Barton RA, Harvey PH (2000). Mosaic evolution of brain structure in mammals. Nature 405:1055-1058. 
Baxter MG, Murray EA (2002). The amygdala and reward. Nature Rev Neurosci 3:563573.

Beamer WG, Shultz KL, Donahue LR, Churchill GA, Sen S et al. (2001). Quantitative trait loci for femoral and lumbar vertebral bone mineral density in C57BL/6J and $\mathrm{C} 3 \mathrm{H} / \mathrm{HeJ}$ inbred strains of mice. J Bone Miner Res 16:1195-1206.

Beatty J, Laughlin RE (2006). Genomic regulation of natural variation in cortical and noncortical brain volume. BMC Neurosci 7:16.

Beaulieu S, Di Paolo T, Barden N (1986). Control of ACTH secretion by the central nucleus of the amygdala: implication of the serotoninergic system and its relevance to the glucocorticoid delayed negative feedback mechanism. Neuroendocrinology 44: 247-254.

Belzung C, Griebel G (2001). Measuring normal and pathological anxiety-like behaviour in mice: a review. Behav Brain Res 125:141-149.

Bennur S, Shankaranarayana Rao BS, Pawlak R, Strickland S, McEwen BS, Chattarji S (2007). Stress-induced spine loss in the medial amygdala is mediated by tissueplasminogen activator. Neuroscience 144:8-16.

Berton O, Nestler EJ (2006). New approaches to antidepressant drug discovery: beyond monoamines. Nat Rev Neurosci 7:137-151.

Blumber HP, Kaufman J, Martin A, Whiteman R, Zhang JH, Gore JC et al. (2003). Amygdala and hippocampal volumes in adolescents and adults with bipolar disorder. Arch Gen Psychiatry 60:1201-1208.

Bolivar V, Flaherty L (2003). A region on chromosome 15 controls intersession habituation in mice. J Neurosci 23:9435-9438.

Bolivar VJ, Flaherty L (2004). Genetic control of novel food preference in mice. Mamm Genome 15:193-198.

Bonne O, Brandes D, Gilboa A, Gomori JM, Shenton ME, Pitman RK et al. (2001). Longitudinal MRI study of hippocampal volume in trauma survivors with PTSD. Am J Psychiatry 158:1248-1251.

Bouchard TJ Jr (1994). Genes, environment, and personality. Science 264:1700-1701.

Boyce-Rustay JM, Cameron HA, Holmes A (2007). Chronic swim stress alters sensitivity to acute behavioral effects of ethanol in mice. Physiol Behav 91:77-86.

Boyce-Rustay JM, Holmes A (2006). Genetic inactivation of the NMDA receptor NR2A subunit has anxiolytic- and antidepressant-like effects in mice.

Neuropsychopharmacology 31:2405-2414.

Brem RB, Yvert G, Clinton R, Kruglyak L (2002). Genetic dissection of transcriptional regulation in budding yeast. Science 296:752-755.

Bremner JD, Randall P, Vermetten E, Staib L, Bronen RA, Mazure C et al. (1997). Magnetic resonance imaging-based measurement of hippocampal volume in posttraumatic stress disorder related to childhood physical and sexual abuse - a preliminary report. Biol Psychiatry 41:23-32. 
Brockington M, Torelli S, Prandini P, Boito C, Dolatshad NF, Longman C et al. (2005). Localization and functional analysis of the LARGE family of glycosyltransferase: significance for muscular dystrophy. Hum Mol Genet 14:657-665.

Brunner HG, Nelen M, Breakefield XO, Ropers HH, van Oost BA (1993). Abnormal behavior associated with a point mutation in the structural gene for monoamine oxidase A. Science 262:578-580.

Buck K, Metten P, Belknap J, Crabbe J (1999). Quantitative trait loci affecting risk for pentobarbital withdrawal map near alcohol withdrawal loci on mouse chromosomes 1, 4, and 11. Mamm Genome 10:431-437.

Buck KJ, Metten P, Belknap JK, Crabbe JC (1997). Quantitative trait loci involved in genetic predisposition to acute alcohol withdrawal in mice. J Neurosci 17:3946-3955.

Buono RJ, Lohoff FW, Sander T, Sperling MR, O'Connor MJ, Dlugos DJ et al. (2004). Association between variation in the human KCNJ10 potassium ion channel gene and seizure susceptibility. Epilepsy Res 58:175-183.

Bystrykh L, Weersing E, Dontje B, Sutton S, Pletcher MT, Wiltshire T et al. (2005). Uncovering regulatory pathways that effect hematopoietic stem cell function using 'genetical genomics'. Nat Genet 37:225-232.

Cai L, Morrow EM, Cepko CL (2000). Misexpression of basic helix-loop-helix genes in the murine cerebral cortex affects cell fate choices and neuronal survival. Development 127:3021-3030.

Caldarone B, Saavedra C, Tartaglia K, Wehner JM, Dudek BC, Flaherty L (1997). Quantitative trait loci analysis affecting contextual conditioning in mice. Nat Genet $17: 335-337$.

Calder AJ, Lawrence AD, Young AW (2001). Neuropsychology of fear and loathing. Nature Rev Neurosci 2:352-363.

Carpentier R, Sacchetti P, Ségard P, Staels B, Lefebvre P (2008). The glucocorticoid receptor is a co-regulator of the orphan nuclear receptor Nurr1. J Neurochem 104:777789.

Casley WL, Menzies JA, Whitehouse LW, Moon TW (1999). Detection of quantitative trait loci affecting caffeine metabolism by interval mapping in a genome-wide scan of C3H/HeJ x APN F(2) mice. Drug Metab Dispos 27:1375-1380.

Caspi A, Moffitt TE (2006). Gene-environment interactions in psychiatry: joining forces with neuroscience. Nat Rev Neurosci 7:583-590.

Crawley JN (1981). Neuropharmacologic specificity of a simple animal model for the behavioral actions of benzodiazepines. Pharmacol Biochem Behav 15:695-699.

Charney DS, Manji HK (2004). Life stress, genes, and depression: multiple pathways lead to increased risk and new opportunities for intervention. Sci STKE 2004:re5.

Chesler EJ, Lu, L, Shou, S, Qu Y, Gu J, Wang J et al. (2005). Complex trait analysis of gene expression uncovers polygenic and pleiotropic networks that modulate nervous system function. Nat Genet 37:233-242. 
Chesler EJ, Wang J, Lu L, Qu Y, Manly KF, Williams RW (2003). Genetic correlates of gene expression in recombinant inbred strains: a relational model system to explore neurobehavioral phenotypes. Neuroinformatics 1:343-357.

Cowles CR, Hirschhorn JN, Altshuler D, Lander ES (2002). Detection of regulatory variation in mouse genes. Nat Genet 32:432-437.

Crabbe J (1996). Quantitative trait locus gene mapping: a new method for locating alcohol response genes. Addict Biol 1:229-235.

Crabbe JC, Kosobud A, Young ER, Janowsky JS (1983). Polygenic and single-gene determination of responses to ethanol in $\mathrm{BXD} / \mathrm{Ty}$ recombinant inbred mouse strains. Neurobehav Toxicol Teratol 5:181-187.

Cryan CF, Dev KK (2007). The glutamatergic system as a potential therapeutic target for the treatment of anxiety disorders. Handbook of Anxiety and Fear. (Blanchard RJ, Blanchard DC, Nutt DJ, eds) Elsevier, Amsterdam.

Cryan JF, Holmes A (2005). The ascent of mouse: advances in modelling human depression and anxiety. Nat Rev Drug Discov 4:775-790.

Cui H, Bulleit RF (1998). Expression of the POU transcription factor Brn-5 is an early event in the terminal differentiation of CNS neurons. J Neurosci Res 15:625-632.

Cunningham CL (1995). Localization of genes influencing ethanol-induced conditioned place preference and locomotor activity in BXD recombinant inbred mice.

Psychopharmacology (Berl) 120:28-41.

Damerval C, Maurice A, Josse JM, de Vienne D (1994). Quantitative trait loci underlying gene product variation: a novel perspective for analyzing regulation of genome expression. Genetics 137:289-301.

Davis M, Whalen PJ (2001). The amygdala: vigilance and emotion. Mol Psychiatry 6:1334.

De Bellis MD, Hall J, Boring AM, Frustaci K, Moritz G (2001). A pilot longitudinal study of hippocampal volumes in pediatric maltreatment-related posttraumatic stress disorder. Biol Psychiatry 50:305-309.

De Bellis MD, Keshavan MS, Shifflett H, Iyengar S, Beers SR, Hall J et al. (2002). Brain structures in pediatric maltreatment-related posttraumatic stress disorder: a sociodemographically matched study. Biol Psychiatry 52:1066-1078.

de Kloet CS, Vermetten E, Geuze E, Kavelaars A, Heijnen CJ, Westenberg HG (2006). Assessment of HPA-axis function in posttraumatic stress disorder: pharmacological and nonpharmacological challenge tests, a review. J Psychiatr Res 40:550-567.

de la Fuente A, Bing N, Hoeschele I, Mendes P (2004). Discovery of meaningful associations in genomic data using partial correlation coefficients. Bioinformatics 20:3565-3574.

de Vienne D, Maurice A, Josse JM, Leonardi A, Damerval C (1994). Mapping factors controlling genetic expression. Cell Mol Biol (Noisy-le-grand) 40:29-39. 
Decker MW, Curzon P, Brioni JD (1995). Influence of separate and combined septal and amygdala lesions on memory, acoustic startle, anxiety, and locomotor activity in rats. Neurobiol Learn Mem 64:156-168.

DeFries JC, Gervais MC, Thomas EA (1978). Response to 30 generations of selection for open-field activity in laboratory mice. Behav Genet 129:1432-1435.

Demarest K, Koyner J, McCaughran J Jr, Cipp L, Hitzemann R (2001). Further characterization and high-resolution mapping of quantitative trait loci for ethanol-induced locomotor activity. Behav Genet 31:79-91.

Dennis G, Jr., Sherman BT, Hosack DA, Yang J, Gao W, Lane HC, Lempicki RA (2003). DAVID: Database for Annotation, Visualization, and Integrated Discovery. Genome Biol 4:P3.

Di Ciano P, Everitt BJ (2004). Direct interactions between the basolateral amygdala and nucleus accumbens core underlie cocaine-seeking behavior by rats. J. Neurosci 24:71677173.

Ding Q, Cecarini V, Keller JN (2007). Interplay between protein synthesis and degradation in the CNS: physiological and pathological implications. Trends Neurosci 30:31-36.

Dittmar KA, Goodenbour JM, Pan T (2006). Tissue-specific differences in human transfer RNA expression. PLoS Genet 2:e221.

Drenan RM, Doupnik CA, Boyle MP, Muglia LJ, Huettner JE et al. (2005).

Palmitoylation regulates plasma membrane-nuclear shuttling of R7BP, a novel membrane anchor for the RGS7 family. J Cell Biol 169:623-633.

Drevets WC (2000). Functional anatomical abnormalities in limbic and prefrontal cortical structures in major depression. Prog Brain Res 126:413-431.

Drews VL, Shi K, de Haan G, Meisler MH (2007). Identification of evolutionarily conserved, functional noncoding elements in the promoter region of the sodium channel gene SCN8A. Mamm Genome 18:723-731.

Du W, Bautista JF, Yang H, Diez-Sampedro A, You SA et al. (2005). Calcium-sensitive potassium channelopathy in human epilepsy and paroxysmal movement disorder. Nat Genet 37:733-738.

Duchaîne TF, Hemraj I, Furic L, Deitinghoff A, Kiebler MA et al. (2002). Staufen2 isoforms localize to the somatodendritic domain of neurons and interact with different organelles. J Cell Sci 115:3285-3295.

Dulawa SC, Holick KA, Gundersen B, Hen R (2004). Effects of chronic fluoxetine in animal models of anxiety and depression. Neuropsychopharmacology 29:1321-1330.

Dunn JD, Whitener J (1986). Plasma corticosterone responses to electrical stimulation of the amygdaloid complex: cytoarchitectural specificity. Neuroendocrinology 42:211-217.

Eberwine J, Belt B, Kacharmina JE, Miyashiro K (2002). Analysis of subcellularly localized mRNAs using in situ hybridization, mRNA amplification, and expression profiling. Neurochem Ress 27:1065-1077. 
Einat H, Manji HK (2006). Cellular plasticity cascades: genes-to-behavior pathways in animal models of bipolar disorder. Biol Psychiatry 59:1160-1171.

Emmons S, Phan H, Calley J, Chen W, James B, Manseau L (1995). Cappuccino, a Drosophila maternal effect gene required for polarity of the egg and embryo, is related to the vertebrate limb deformity locus. Genes Dev 9:2482-2494.

Eppig JT, Bult CJ, Kadin JA, Richardson JE, Blake JA, Anagnostopoulos A et al. (2005). The Mouse Genome Database (MGD): from genes to mice - a community resource for mouse biology. Nucleic Acids Res 33:D471-D475.

Falconer DS, Mackay TFC (1996). Introduction to Quantitative Genetics, 4th edn. Prentice Hall, Harlow, UK.

Fanselow MS, LeDoux JE (1999). Why we think plasticity underlying Pavlovian fear conditioning occurs in the basolateral amygdala. Neuron 23:229-232.

Fanselow MS, Poulos AM (2005). The neuroscience of mammalian associative learning. Annu Rev Psychol 56:207-234.

Fedele DE, Li T, Lan JQ, Fredholm BB, Boison D (2006). Adenosine A1 receptors are crucial in keeping an epileptic focus localized. Exp Neurol 200:184-190.

Fennema-Notestine C, Stein MB, Kennedy CM, Archibald SL, Jernigan TL (2002). Brain morphometry in female victims of intimate partner violence with and without posttraumatic stress disorder. Biol Psychiatry 52:1089-1101.

Ferraro TN, Golden GT, Dahl JP, Smith GG, Schwebel CL et al. (2007). Analysis of a quantitative trait locus for seizure susceptibility in mice using bacterial artificial chromosome-mediated gene transfer. Epilepsia 48:1667-1677.

Ferraro TN, Golden GT, Smith GG, Martin JF, Lohoff FW, Gieringer TA et al. (2004). Fine mapping of a seizure susceptibility locus on mouse Chromosome 1: nomination of Kcnj10 as a causative gene. Mamm Genome 15:239-251.

Flint J (2003). Analysis of quantitative trait loci that influence animal behavior. J Neurobiol 54:46-77.

Flint J, Corley R, DeFries JC, Fulker DW, Gray JA, Miller S et al. (1995). A simple genetic basis for a complex psychological trait in laboratory mice. Science 269:14321435.

Floyd DW, Jung KY, McCool BA (2003). Chronic ethanol ingestion facilitates Nmethyl-D-aspartate receptor function and expression in rat lateral/basolateral amygdala neurons. J Pharmacol Exp Ther. 307:1020-1029.

Franklin KBJ, Paxinos G (2001). The Mouse Brain in Stereotaxic Coordinates. Academic Press, San Diego.

Friedman DP, Aggleton JP, Saunders RC (2002). Comparison of hippocampal, amygdala, and perirhinal projections to the nucleus accumbens: combined anterograde and retrograde tracing study in the Macaque brain. J Comp Neurol 450:345-365. 
Fuchs RA, Feltenstein MW, See RE (2006). The role of the basolateral amygdala in stimulus-reward memory and extinction memory consolidation and in subsequent conditioned cues reinstatement of cocaine seeking. Eur J Neurosci 23:2809-2813.

Gatti D, Maki A, Chesler EJ, Kirova R, Kosyk O, Lu L et al. (2007). Genome-level analysis of genetic regulation of liver gene expression networks. Hepatology 46:548-557. Gerlai R (1996). Gene-targeting studies of mammalian behavior: is it the mutation or the background genotype? Trends Neurosci 19:177-181.

Gershenfeld HK, Neumann PE, Mathis C, Crawley JN, Li X, Paul SM (1997). Mapping quantitative trait loci for open-field behavior in mice. Behav Genet 27:201-210.

Gershenfeld HK, Paul SM (1998). Towards a genetics of anxious temperament: from mice to men. Acta Psychiatr Scand Suppl 393:56-65.

Gilbertson MW, Shenton ME, Ciszewski A, Kasai K, Lasko NB, Orr SP et al. (2002). Smaller hippocampal volume predicts pathologic vulnerability to psychological trauma. Nat Neurosci 5:1242-1247.

Goldman D, Oroszi G, Ducci F (2005). The genetics of addictions: uncovering the genes. Nat Rev Genet 6:521-532.

Goosens KA, Maren S (2001). Contextual and auditory fear conditioning are mediated by the lateral, basal, and central amygdaloid nuclei in rats. Learn Mem 8:148-155.

Govindarajan A, Rao BS, Nair D, Trinh M, Mawjee N, Tonegawa S, Chattarji S (2006). Transgenic brain-derived neurotrophic factor expression causes both anxiogenic and antidepressant effects. Proc Natl Acad Sci U S A 103:13208-13213.

Graubert TA, Cahan P, Edwin D, Selzer RR, Richmond TA et al. (2007). A highresolution map of segmental DNA copy number variation in the mouse genome. PLoS Genet 3:e3.

Grice DE, Reenilä I, Männistö PT, Brooks AI, Smith GG, Golden GT et al. (2007). Transcriptional profiling of C57 and DBA strains of mice in the absence and presence of morphine. BMC Genomics 8:76.

Gross SR, Kinzy TG (2007). Improper organization of the actin cytoskeleton affects protein synthesis at initiation. Mol Cell Biol 27:1974-1989.

Guadaño-Ferraz A, Benavides-Piccione R, Venero C, Lancha C, Vennström B, Sandi C et al. (2003). Lack of thyroid hormone receptor $\alpha 1$ is associated with selective alterations in behavior and hippocampal circuits. Mol Psychiatry 8:30-38.

Gundersen HJG (1977). Notes on the estimation of the numerical density of arbitrary profiles: the edge effect. J Micros 111:21-23.

Gurvits TV, Metzger LJ, Lasko NB, Cannistraro PA, Tarhan AS, Gilbertson MW et al. (2006). Subtle neurologic compromise as a vulnerability factor for combat-related posttraumatic stress disorder: results of a twin study. Arch Gen Psychiatry 63:571-576.

Hains AB, Arnsten AF (2008). Molecular mechanisms of stress-induced prefrontal cortical impairment: implications for mental illness. Learn Mem 15:551-564. 
Hajek T, Carrey N, Alda M (2005). Neuroanatomical abnormalities as risk factors for bipolar disorder. Bipolar Disord 7:393-403.

Haley CS, Knott SA (1992). A simple regression method for mapping quantitative trait loci in line crosses using flanking markers. Heredity 69:315-324.

Handley SL, Mithani S (1984). Effects of alpha-adrenoceptor agonistsand antagonists in a maze-exploration model of 'fear'-motivated behaviour. Naunyn Schmiedebergs Arch Pharmacol 327:1-5.

Hariri AR, Drabant EM, Weinberger DR (2006). Imaging genetics: perspectives from studies of genetically driven variation in serotonin function and corticolimbic affective processing. Biol Psychiatry 59:888-897.

Hariri AR, Holmes A (2006). Genetics of emotional regulation: the role of the serotonin transporter in neural function. Trends Cogn Sci 10:182-191.

Hayes DM, Knapp DJ, Breese GR, Thiele TE (2005). Comparison of basal neuropeptide $\mathrm{Y}$ and corticotropin releasing factor levels between the high ethanol drinking C57BL/6J and low ethanol drinking DBA/2J inbred mouse strains. Alcohol Clin Exp Res 29:721729.

Hefner K, Holmes A (2007). Ontogeny of fear-, anxiety- and depression-related behavior across adolescence in C57BL/6J mice. Behav Brain Res 176:210-215.

Hefner K, Whittle N, Juhasz J, Norcross M, Karlsson RM, Saksida LM et al. (2008). Impaired fear extinction learning and cortico-amygdala circuit abnormalities in a common genetic mouse strain. J Neurosci 28:8074-8085.

Henshall DC, Araki T, Schindler CK, Lan JQ, Tiekoter KL, Taki W et al. (2002). Activation of Bcl-2-associated death protein and counter-response of Akt within cell populations during seizure-induced neuronal death. J Neurosci 22:8458-8465.

Henshall DC, Schindler CK, So NK, Lan JQ, Meller R, Simon RP (2004). Deathassociated protein kinase expression in human temporal lobe epilepsy. Ann Neurol 55:485-494.

Herman JP, Ostrander MM, Mueller NK, Figueiredo H (2005). Limbic system mechanisms of stress regulation: hypothalamo-pituitary-adrenocortical axis. Prog Neuropsychopharmacol Biol Psychiatry 29:1201-1213.

Herry C, Bach DR, Esposito F, Di Salle F, Perrig WJ, Scheffler K et al. (2007). Processing of temporal unpredictability in human and animal amygdala. J Neurosci 27:5958-5966.

Hitzemann R, Malmanger B, Cooper S, Coulombe S, Reed C, Demarest K et al. (2002). Multiple cross mapping (MCM) markedly improves the localization of a QTL for ethanol-induced activation. Genes Brain Behav 1:214-222.

Hitzemann R, Malmanger B, Reed C, Lawler M, Hitzemann B, Coulombe S et al. (2003). A strategy for the integration of QTL, gene expression, and sequence analyses. Mamm Genome 14:733-747. 
Hitzemann R, Reed C, Malmanger B, Lawler M, Hitzemann B, Cunningham B et al. (2004). On the integration of alcohol-related quantitative trait loci and gene expression analyses. Alcohol Cln Exp Res 28:1437-1448.

Holmes A, Kinney JW, Wrenn CC, Li Q, Yang RJ, Ma L et al. (2003). Galanin GAL-R1 receptor null mutant mice display increased anxiety-like behavior specific to the elevated plus-maze. Neuropsychopharmacology 28:1031-1044.

Holmes A, le Guisquet AM, Vogel E, Millstein RA, Leman S, Belzung C (2005). Early life genetic, epigenetic and environmental factors shaping emotionality in rodents. Neurosci Biobehav Rev 29:1335-1346.

Holmes A, Wellman CL (2009). Stress-induced prefrontal reorganization and executive dysfunction in rodents. Neurosci Biobehav Rev [Epub ahead of print]

Holmes A, Yang RJ, Lesch KP, Crawley JN, Murphy DL (2003). Mice lacking the serotonin transporter exhibit 5-HT(1A) receptor-mediated abnormalities in tests for anxiety-like behavior. Neuropsychopharmacology 28:2077-2088.

Hosack DA, Dennis G Jr, Sherman BT, Lane HC, Lempicki RA (2003). Identifying biological themes within lists of genes with EASE. Genome Biol 4:R70.

Hovatta I, Barlow C (2008). Molecular genetics of anxiety in mice and men. Ann Med 40:92-109.

Hui GK, Figueroa IR, Poytress BS, Roozendaal B, McGaugh JL, Weinberger NM (2004). Memory enhancement of classical fear conditioning by post-training injections of corticosterone in rats. Neurobiol Learn Mem 81:67-74.

Hunt RA, Edris W, Chanda PK, Nieuwenhuijsen B, Young KH (2003). Snapin interacts with the N-terminus of regulator of $\mathrm{G}$ protein signaling 7. Biochem Biophys Res Commun 303:594-599.

Ida M, Shuo T, Hirano K, Tokita Y, Nakanishi K, Matsui F et al. (2006). Identfication and function of chondroitin sulfate in the milieu of neural stem cells. J Biol Chem 281:5982-5991.

Ikeda K, Onaka T, Yamakado M, Nakai J, Ishikawa TO, Taketo MM et al. (2003). Degeneration of the amygdala/piriform cortex and enhanced fear/anxiety behaviors in sodium pump alpha2 subunit (Atp1a2)-deficient mice. J Neurosci 23:4667-4676.

Ingram VM (1958). Abnormal human haemoglobins. I. The comparison of normal human and sickle-cell haemoglobins by fingerprinting. Biochim Biophys Acta 28:539-545.

Izquierdo A, Wellman CL, Holmes A (2006). Brief uncontrollable stress causes dendritic retraction in infralimbic cortex and resistance to fear extinction in mice. J Neurosci 26:5733-5738.

Jacobson LH, Cryan JF (2007). Feeling strained? Influence of genetic background on depression-related behavior in mice: a review. Behav Genet 37:171-213.

Johnstone O, Lasko P (2001). Translational regulation and RNA localization in Drosophila oocytes and embryos. Annu Rev Genet 35:365-406. 
Jones BC, Tarantino LM, Rodriguez LA, Reed CL, McClearn GE, Plomin R et al. (1999). Quantitative-trait loci analysis of cocaine-related behaviours and neurochemistry. Pharmacogenetics 9:607-617.

Karl A, Schaefer M, Malta LS, Dorfel D, Rohleder N, Werner A (2006). A meta-analysis of structural brain abnormalities in PTSD. Neurosci Biobehav Rev 30:1004-1031.

Karlsson RM, Holmes A (2006). Galanin as a modulator of anxiety and depression and a therapeutic target for affective disease. Amino Acids 31:231-239.

Karlsson RM, Tanaka K, Heilig M, Holmes A (2008). Loss of glial glutamate and aspartate transporter (excitatory amino acid transporter 1) causes locomotor hyperactivity and exaggerated responses to psychotomimetics: rescue by haloperidol and metabotropic glutamate 2/3 agonist. Biol Psychiatry 64:810-814.

Katsanis N, Fitzgibbon J, Fisher EM (1996). Paralogy mapping: identification of a region in the human MHC triplicated onto human chromosomes 1 and 9 allows the prediction and isolation of novel PBX and NOTCH loci. Genomics 35:101-108.

Kempermann G, Chesler EJ, Lu L, Williams RW, Gage FH (2006). Natural variation and genetic covariance in adult hippocampal neurogenesis. Proc Natl Acad Sci USA 103:780-785.

Kendler KS (2001). Twin studies of psychiatric illness: an update. Arch Gen Psychiatry 58:1005-1014.

Kerns RT, Ravindranathan A, Hassan S, Cage MP, York T, Sikela JM et al. (2005). Ethanol-responsive brain region expression networks: implications for behavioral responses to acute ethanol in DBA/2J versus C57BL/6J mice. J Neurosci 25:2255-2266.

Kim JJ, Fanselow MS (1992). Modality-specific retrograde amnesia of fear. Science 256:675-677.

Kliethermes CL, Crabbe JC (2006). Genetic independence of mouse measures of some aspects of novelty seeking. Proc Natl Acad Sci USA 103:5018-5023.

Koo JW, Han JS, Kim JJ (2004). Selective neurotoxic lesions of basolateral and central nuclei of the amygdala produce differential effects on fear conditioning. J Neurosci 24:7654-7662.

Koob GF (2003). Alcoholism: allostasis and beyond. Alcohol Clin Exp Res 27:232-243.

Korostynski M, Kaminska-Chowaniec D, Piechota M, Przewlocki R (2006). Gene expression profiling in the striatum of inbred mouse strains with distinct opioid-related phenotypes. BMC Genomics 7:146.

Krishnan V, Han MH, Graham DL, Berton O, Renthal W, Russo SJ et al. (2007). Molecular adaptations underlying susceptibility and resistance to social defeat in brain reward regions. Cell 131:391-404.

Labarca C, Schwarz J, Deshpande P, Schwarz S, Nowak MW, Fonck C et al. (2001). Point mutant mice with hypersensitive alpha 4 nicotinic receptors show dopaminergic deficits and increased anxiety. Proc Natl Acad Sci U S A 98:2786-2791. 
Lau C, Ng L, Thompson C, Pathak S, Kuan L, Jones A et al. (2008). Exploration and visualization of gene expression with neuroanatomy in the adult mouse brain. BMC Bioinformatics 9:153.

Leader B, Leder P (2000). Formin-2, a novel formin homology protein of the cappuccino subfamily, is highly expressed in the developing and adult central nervous system. Mech Dev 93:221-231.

Leader B, Lim H, Carabatsos MJ, Harrington A, Ecsedy J, Pellman D et al. (2002). Formin-2, polyploidy, hypofertility and positioning of the meiotic spindle in mouse oocytes. Nat Cell Biol 4:921-928.

LeDoux JE (2000). Emotion circuits in the brain. Annu Rev Neurosci 23:155-184.

Lee GP, Meador KJ, Loring DW, Allison JD, Brown WS, Paul LK et al. (2004). Neural substrates of emotion as revealed by functional magnetic resonance imaging. Cog Behav Neurol 17:9-17.

Legare ME, Frankel WN (2000). Multiple seizure susceptibility genes on chromosome 7 in SWXL-4 congenic mouse strains. Genomics 70:62-65.

Lein ES, Hawrylycz MJ, Ao N, Ayres M, Bensinger A, Bernard A et al. (2007). Genomewide atlas of gene expression in the adult mouse brain. Nature 445:168-176.

Le-Niculescu H, McFarland MJ, Ogden CA, Balaraman Y, Patel S, Tan J et al. (2008). Phenomic, convergent functional genomic, and biomarker studies in a stress-reactive genetic animal model of bipolar disorder and co-morbid alcoholism. Am J Med Genet B Neuropsychiatr Genet 147B:134-166.

Li CX, Wei X, Lu, L, Peirce JL, Williams RW, Waters RS (2005). Genetic analysis of barrel field size in the first somatosensory area $(\mathrm{S} 1)$ in inbred and recombinant inbred strains of mice. Somatosens Mol Res 22:141-150.

Li J, Jiang T, Mao JH, Balmain A, Peterson L, Harris C et al. (2004). Genomic segmental polymorphisms in inbred mouse strains. Nat Genet 36:952-954.

Lin CH, Hansen S, Wang Z, Storm DR, Tapscott SJ, Olson JM (2005). The dosage of the neuroD2 transcription factor regulates amygdala development and emotional learning. Proc Natl Acad Sci USA 102:14877-14882.

Lindauer RJ, Vlieger EJ, Jalink M, Olff M, Carlier IV, Majoie CB et al. (2004). Smaller hippocampal volume in Dutch police officers with posttraumatic stress disorder. Biol Psychiatry 56:356-363.

Ling EA, Paterson JA, Privat A, Mori S, Leblond CP (1973). Investigation of glial cells in semi-thin sections. I. Identification of glial cells in the brain of young rats. J Comp Neurol 149:43-71.

Livak KJ, Schmittgen TD (2001). Analysis of relative gene expression data using realtime quantitative PCR and the 2(-Delta Delta C(T)) Method. Methods 25:402-408.

Lowe TM, Eddy SR (1997). tRNAscan-SE: a program for improved detection of transfer RNA genes in genomic sequence. Nucleic Acids Res 25:955-964. 
Lu L, Airey DC, Williams RW (2001). Complex trait analysis of the hippocampus: mapping and biometric analysis of two novel gene loci with specific effects on hippocampal structure in mice. J Neurosci 21:3503-3514.

Lynch M, Walsh B (1998). Genetics and Analysis of Quantitative traits. Sinauer, Sunderland MA.

Malenka RC, Bear MF (2004). LTP and LTD: an embarrassment of riches. Neuron 44:521.

Malgaroli A, Vallar L, Zimarino V (2006). Protein homeostasis in neurons and its pathological alterations. Curr Opin Neurobiol 16:270-274.

Maren S, Quirk GJ (2004). Neuronal signaling of fear memory. Nat Rev Neurosci 5: 844-852.

Maret S, Dorsaz S, Gurcel L, Pradervand S, Petit B, Pfister C et al. (2007). Homerla is a core brain molecular correlate of sleep loss. Proc Natl Acad Sci U S A 104:20090-20095.

Matthews DB, Bhave SV, Belknap JK, Brittingham C, Chesler EJ, Hitzemann RJ et al. (2005). Complex genetics of interactions of alcohol and CNS function and behavior. Alcohol Clin Exp Res 29:1706-1719.

Maywood ES, O'Neill JS, Reddy AB, Chesham JE, Prosser HM, Kyriacou CP et al. (2007). Genetic and molecular analysis of the central and peripheral circadian clockwork of mice. Cold Spring Harb Symp Quant Biol 72:85-94.

McBride WJ (2002). Central nucleus of the amygdala and the effects of alcohol and alcohol-drinking behavior in rodents. Pharmacol Biochem Behav 71:509-515.

McClung CA (2007). Circadian genes, rhythms and the biology of mood disorders. Pharmacol Ther 114:222-232.

McGaugh JL (2003). The amygdala modulates the consolidation of memories of emotionally arousing experiences. Annu Rev Neurosci 27:1-28.

McIntyre CK, Power AE, Roozendaal B, McGaugh JL (2003). Role of the basolateral amygdala in memory consolidation. Ann N Y Acad Sci 985:273-293.

Meyer-Lindenberg A, Buckholtz JW, Kolachana B, Hariri A, PezawasL, Blasi G et al. (2006). Neural mechanisms of genetic risk for impulsivity and violence in humans. Proc Natl Acad Sci USA 103:6269-6274.

Mills PB, Surtees RA, Champion MP, Beesley CE, Dalton N, Scambler PJ et al. (2005). Neonatal epileptic encephalopathy caused by mutations in the PNPO gene encoding pyridox(am).ine 5'-phosphate oxidase. Hum Mol Genet 14:1077-1086.

Millstein RA, Holmes A (2007). Effects of repeated maternal separation on anxiety- and depression-related phenotypes in different mouse strains. Neurosci Biobehav Rev 31:317.

Mineur YS, Belzung C, Crusio WE (2006). Effects of unpredictable chronic mild stress on anxiety and depression-like behavior in mice. Behav Brain Res 175:43-50. 
Mitra R, Jadhav S, McEwen BS, Vyas A, Chattarji S (2005). Stress duration modulates the spatiotemporal patterns of spine formation in the basolateral amygdala. Proc Natl Acad Sci USA 102:9371-9376.

Moller C, Wiklund L, Sommer W, Thorsell A, Heilig M (1997). Decreased experimental anxiety and voluntary ethanol consumption in rats following central but not basolateral amygdala lesions. Brain Res 760:94-101.

Moreira CM, Masson S, Carvalho MC, Brandao ML (2007). Exploratory behaviour of rats in the elevated plus-maze is differentially sensitive to inactivation of the basolateral and central amygdaloid nuclei. Brain Res Bull 71:466-474.

Mozhui K, Ciobanu DC, Schikorski T, Wang X, Lu L, Williams RW (2008). Dissection of a QTL hotspot on mouse distal chromosome 1 that modulates neurobehavioral phenotypes and gene expression. PLoS Genet 4:e1000260.

Mozhui K, Hamre KM, Holmes A, Lu L, Williams RW (2007). Genetic and structural analysis of the basolateral amygdala complex in BXD recombinant inbred mice. Behav Genet 37:223-243.

Nader K, Majidishad P, Amorapanth P, LeDoux JE (2001). Damage to the lateral and central, but not other, amygdaloid nuclei prevents the acquisition of auditory fear conditioning. Learn Mem 8:156-163.

Nestler EJ, Barrot M, DiLeone RJ, Eisch AJ, Gold SJ, Monteggia LM (2002). Neurobiology of depression. Neuron 34:13-25.

Neumann PE, Garretson JD, Skabardonis GP, Mueller GG (1993). Genetic analysis of cerebellar folial pattern in crosses of $\mathrm{C} 57 \mathrm{BL} / 6 \mathrm{~J}$ and $\mathrm{DBA} / 2 \mathrm{~J}$ inbred mice. Brain Res 619:81-88.

Niu L, Matsui M, Zhou SY, Hagino H, Takahashi T, Yoneyama E et al. (2004). Volume reduction of the amygdala in patients with schizophrenia: a magnetic resonance imaging study. Psychiatry Res 132:41-51.

Norcross M, Mathur P, Enoch AJ, Karlsson RM, Brigman J, Cameron HA et al. (2008). Fluoxetine treatment during adolescence does not cause lasting deficits in fear-, anxietyor stress-related behaviors in mice. Psychopharmacology (Berl) [Epub ahead of print]

Oohira A, Matsui F, Tokita Y, Yamauchi S, Aono S (2000). Molecular interactions of neural chondroitin sulfate proteoglycans in the brain development. Arch Biochem Biophys 374:24-34.

Owen EH, Christensen SC, Paylor R, Wehner JM (1997). Identification of quantitative trait loci involved in contextual and auditory-cued fear conditioning in BXD recombinant inbred strains. Behav Neurosci 111:292-300.

Paigen K, Szatkiewicz JP, Sawyer K, Leahy N, Parvanov ED, Ng SH et al. (2008). The recombinational anatomy of a mouse chromosome. PLoS Genet 4:e1000119.

Palmer AA, Miller MN, McKinnon CS, Phillips TJ (2002). Sensitivity to the locomotor stimulant effects of ethanol and allopregnanolone is influenced by common genes. Behav Neurosci 116:126-137. 
Papaleo F, Crawley JN, Song J, Lipska BK, Pickel J, Weinberger DR et al. (2008). Genetic dissection of the role of catechol-O-methyltransferase in cognition and stress reactivity in mice. J Neurosci 28:8709-8723.

Patel NV, Hitzemann RJ (1999). Detection and mapping of quantitative trait loci for haloperidol-induced catalepsy in a C57BL/6J x DBA/2J F2 intercross. Behav Genet 29:303-310.

Pauling L, Itano HA, Singer SJ, Wells IC (1949). Sickle cell anemia, a molecular disease. Science 110:543-548.

Peirce JL, Chesler EJ, Williams RW, Lu L (2003). Genetic architecture of the mouse hippocampus: identification of geneloci with selective regional effects. Genes Brain Behav 2:238-252.

Peirce JL, Li H, Wang J, Manly KF, Hitzemann RJ, Belknap JK et al. (2006). How replicable are mRNA expression QTL? Mamm Genome 17:643-656.

Peirce JL, Lu L, Gu J, Silver LM, Williams RW (2004). A new set of BXD recombinant inbred lines from advanced intercross population in mice. BMC Genetics 5:7.

Peirce JL, Williams RW (2006). Expression and brain structure: black boxes between genes and behaviors. Neurobehavioral Genetics: Methods and Applications, 2nd edn. (Jones B, Mormede P, eds) Taylor and Francis, Boca Raton.

Peri T, Ben-Shakhar G, Orr SP, Shalev AY (2000). Psychophysiologic assessment of aversive conditioning in posttraumatic stressdisorder. Biol Psychiatry 47:512-519.

Petkov PM, Graber JH, Churchill GA, DiPetrillo K, King BL, Paigen K (2006). Evidence of a large-scale functional organization of mammalian chromosomes. PLoS Genet 1:e33.

Pezawas L, Meyer-Lindenberg A, Drabant EM, Verchinski BA, Munoz KE, Kolachana BS et al. (2005). 5-HTTLPR polymorphism impacts human cingulated-amygdala interactions: a genetic susceptibility mechanism for depression. Nat Neurosci 8:828-834.

Phelps EA, LeDoux JE (2005). Contributions of the amygdala to emotion processing: from animal models to human behavior. Neuron 48:175-187.

Phillips AG, Ahn S, Howland JG (2003). Amygdalar control of the meso-corticolimbic dopamine system: parallel pathways to motivated behavior. Neurosci Biobehav Rev 27:543-554.

Phillips TJ, Huson MG, McKinon CS (1998). Localization of genes mediating acute and sensitized locomotor responses to cocaine in $\mathrm{BXD} / \mathrm{Ty}$ recombinant inbred mice. $\mathrm{J}$ Neurosci 18:3023-3034.

Phillips TJ, Lessov CN, Harland RD, Mitchell SR (1996). Evaluation of potential genetic associations between ethanol tolerance and sensitization in BXD/Ty recombinant inbred mice. J Pharmacol Exp Ther 277:613-623.

Pitkänen A (2000). Connectivity of the rat amygdaloid complex. The Amygdala, 2nd edn. (Aggleton JP, ed) Oxford University Press Inc., New York. 
Pitkänen A, Savander V, LeDoux JE (1997). Organization of intra-amygdaloid circuitries in the rat: an emerging framework for understanding functions of the amygdala. Trends Neurosci 20:517-523.

Poon MM, Choi SH, Jamieson CA, Geschwind DH, Martin KC (2006). Identification of process-localized mRNAs from cultured rodent hippocampal neurons. J Neurosci 26:13390-13399.

Porsolt RD, Bertin A, Jalfre M (1978). 'Behavioural despair' in rats and mice: strain differences and the effects of imipramine. Eur J Pharmacol 51:291-294.

Porsolt RD, Le Pichon M, Jalfre M (1977). Depression: a new animal model sensitive to antidepressant treatments. Nature 266:730-732.

Pothion S, Bizot JC, Trovero F, Belzung C (2004). Strain differences in sucrose preference and in the consequences of unpredictable chronic mild stress. Behav Brain Res 155:135-146.

Prather MD, Lavenex P, Mauldin-Jourdain ML, Mason WA, Capitanio JP, Mendoza SP et al. (2001). Increased social fear and decreased fear of objects in monkeys with neonatal amygdala lesions. Neuroscience 106:653-658.

Quirk GJ, Mueller D (2007). Neural mechanisms of extinction learning and retrieval. Neuropsychopharmacology 33:56-72.

Radley JJ, Rocher AB, Miller M, Janssen WG, Liston C, Hof PR et al. (2006). Repeated stress induces dendritic spine loss in the rat medial prefrontal cortex. Cereb Cortex 16:313-320.

Ramos A, Mormede P (1998). Stress and emotionality: a multidimensional and genetic approach. Neurosci Biobehav Rev 22:33-57.

Rauch SL, Shin LM, Phelps EA (2006). Neurocircuitry models of posttraumatic stress disorder and extinction: human neuroimaging research - past, present, and future. Biol Psychiatry 60:376-382.

Reddy AB, Maywood ES, Karp NA, King VM, Inoue Y, Gonzalez FJ et al. (2007). Glucocorticoid signaling synchronizes the liver circadian transcriptome. Hepatology 45:1478-1488.

Redgate ES, Fahringer EE (1973). A comparison of the pituitary-adrenal activity elicited by electrical stimulation of preoptic, amygdaloid and hypothalamic sites in the rat brain. Neuroendocrinology 12:334-343.

Reiss AL, Eckert MA, Rose FE, Karchemskiy A, Kesler S, Chang M et al. (2004). An experiment of nature: brain anatomy parallels cognition and behavior in Williams syndrome. J Neurosci 24:5009-5015.

Ressler KJ, Mayberg HS (2007). Targeting abnormal neural circuits in mood and anxiety disorders: from the laboratory to the clinic. Nat Neurosci 10:1116-1124.

Riley B, Kendler KS (2006). Molecular genetic studies of schizophrenia. Eur J Hum Genet 14:669-680. 
Risinger FO, Cunningham CL (1998). Ethanol-induced conditioned taste aversion in BXD recombinant inbred mice. Alcohol Clin Exp Res 22:1234-1244.

Robbins TW, Everitt BJ (2002). Limbic-striatal memory systems and drug addiction. Neurobiol Learn Mem 78:625-636.

Rogan MT, LeDoux JE (1996). Emotion: systems, cells, synaptic plasticity. Cell 85:469475.

Rojas P, Joodmardi E, Hong Y, Perlmann T, Ogren SO (2007). Adult mice with reduced Nurr1 expression: an animal model for schizophrenia. Mol Psychiatry 12:756-766.

Roozendaal B, Hui GK, Hui IR, Berlau DJ, McGaugh JL, Weinberger NM (2006). Basolateral amygdala noradrenergic activity mediates corticosterone-induced enhancement of auditory fear conditioning. Neurobiol Learn Mem 86:249-255.

Rosell DR, Akama KT, Nacher J, McEwen BS (2003). Differential expression of suppressors of cytokine signaling-1, -2 , and -3 in the rat hippocampus after seizure: implications for neuromodulation by gp130 cytokines. Neuroscience 122:349-358.

Rosen GD, La Porte NT, Diechtiareff B, Pung CJ, Nissanov J, Gustafson C et al. (2003). Informatics center for mouse genomics: the dissection of complex traits of the nervous system. Neuroinformatics 1:327-342.

Rosen GD, Pung CJ, Owens CB, Caplow J, Kim H, Mozhui K et al. (2008). Genetic modulation of striatal volume by loci on Chrs 6 and 17 in BXD recombinant inbred mice. Genes Brain Behav [Epub ahead of print]

Rosen GD, Williams RW (2001). Complex trait analysis of the mouse striatum: independent QTLs modulate volume and neuron number. BMC Neurosci 2:5.

Rosen JB (2004). The neurobiology of conditioned and unconditioned fear: a neurobehavioral system analysis of the amygdala. Behav Cogn Neurosci Rev 3:23-41.

Rosso IM, Cintron CM, Steingard RJ, Renshaw PF, Young AD, Yurgelun-Todd DA (2005). Amygdala and hippocampus volumes in pediatric major depression. Biol Psychiatry 57:21-26.

Rosvold HE, Mirsky AF, Pribram KH (1954). Influence of amygdalectomy on social behavior in monkeys. J Comp Physiol Psychol 47:173-178.

Rozen S, Skaletsky H (2000). Primer3 on the WWW for general users and for biologist programmers. Methods Mol Biol 132:365-386.

Ryabinin AE, Wang YM, Freeman P, Risinger FO (1999). Selective effects of alcohol drinking on restraint-induced expression of immediate early genes in mouse brain. Alcohol Clin Exp Res 23:1272-1280.

Ryckelynck M, Giegé R, Frugier M (2005). tRNAs and tRNA mimics as cornerstones of aminoacyl-tRNA synthetase regulations. Biochimie 87:835-845.

Saeed AI, Sharov V, White J, Li J, Liang W, Bhagabati N et al. (2003). TM4: a free, open-source system for microarray data management and analysis. Biotechniques 34:374-378. 
Saitoh O, Kubo Y, Odagiri M, Ichikawa M, Yamagata K, Sekine T (1999). RGS7 and RGS8 differentially accelerate $\mathrm{G}$ protein-mediated modulation of $\mathrm{K}+$ currents. J Biol Chem 274:9899-9904.

Sandberg R, Yasuda R, Pankratz DG, Carter TA, Del Rio JA, Wodicka L et al. (2000). Regional and strain-specific gene expression mapping in the adult mouse brain. Proc Natl Acad Sci USA 97:11038-11043.

Satorre J, Cano J, Reinoso-Suarez F (1986). Quantitative cellular changes during postnatal development of the rat dorsal lateral geniculate nucleus. Anat Embryol 174:321-327.

Schadt EE, Lamb J, Yang X, Zhu J, Edwards S, Guhathakurta D et al. (2005). An integrative genomics approach to infer causal associations between gene expression and disease. Nat Genet 37:710-717.

Schadt EE, Monks SA, Drake TA, Lusis AJ, Che N, Colinayo V et al. (2003). Genetics of gene expression surveyed in maize, mouse and man. Nature 422:297-302.

Schikorski T, Young SM Jr, Hu Y (2007). Horseradish peroxidase cDNA as a marker for electron microscopy in neurons. J Neurosci Methods 165:210-215.

See RE, Fuchs RA, Ledford CC, McLaughlin J (2003). Drug addiction, relapse, and the amygdala. Ann N Y Acad Sci 985:294-307.

Seecharan DJ, Kulkarni AL, Lu L, Rosen GD, Williams RW (2003). Genetic control of interconnected neuronal populations in the mouse primary visual system. J Neurosci 23:11178-11188.

Seyfried TN, Yu RK, Glaser GH (1980). Genetic analysis of audiogenic seizure susceptibility in C57BL/6J X DBA/2J recombinant inbred strains of mice. Genetics 94:701-718.

Shansky RM, Hamo C, Hof PR, McEwen BS, Morrison JH (2009). Stress-induced dendritic remodeling in the prefrontal cortex is circuit specific. Cereb Cortex [Epub ahead of print]

Sharpe AL, Tsivkovskaia NO, Ryabinin AE (2005). Ataxia and c-Fos expression in mice drinking ethanol in a limited access session. Alcohol Clin Exp Res 29:1419-1426.

Shifman S, Bell JT, Copley RR, Taylor MS, Williams RW, Mott R et al. (2006). A highresolution single nucleotide polymorphism genetic map of the mouse genome. PLoS Biol 4:e395.

Shimazoe T, Shibata S, Yatsugi S, Ueki S (1988). Involvement of the medial amygdaloid nucleus in the action of imipramine in rats subjected to the forced swimming test. $\mathrm{J}$ Pharmacobiodyn 11:137-139.

Shin LM, Rauch SL, Pitman RK (2006). Amygdala, medialprefrontal cortex, and hippocampal function in PTSD. Ann N Y Acad Sci 1071:67-79.

Siggins GR, Martin G, Roberto M, Nie Z, Madamba S, De Lecea L (2003). Glutamatergic transmission in opiate and alcohol dependence. Ann N Y Acad Sci 1003:196-211. 
Siu F, Bain PJ, LeBlanc-Chaffin R, Chen H, Kilberg MS (2002). ATF4 is a mediator of the nutrient-sensing response pathway that activates the human asparagine synthetase gene. J Biol Chem 277:24120-24127.

Sklar P, Smoller JW, Fan J, Ferreira MA, Perlis RH, Chambert K et al. (2008). Wholegenome association study of bipolar disorder. Mol Psychiatry 13:558-569.

Smoller JW, Paulus MP, Fagerness JA, Purcell S, Yamaki LH, Hirshfeld-Becker D et al. (2008). Influence of RGS2 on anxiety-related temperament, personality, and brain function. Arch Gen Psychiatry 65:298-308.

Son GH, Chung S, Choe HK, Kim HD, Baik SM, Lee H et al. (2008). Adrenal peripheral clock controls the autonomous circadian rhythm of glucocorticoid by causing rhythmic steroid production. Proc Natl Acad Sci U S A 105:20970-20975.

Song I, Kim D, Choi S, Sun M, Kim Y, Shin HS (2004). Role of the alpha1G T-type calcium channel in spontaneous absence seizures in mutant mice. J Neurosci 24:52495257.

Spanagel R, Pendyala G, Abarca C, Zghoul T, Sanchis-Segura C, Magnone MC et al. (2005). The clock gene Per2 influences the glutamatergic system and modulates alcohol consumption. Nat Med 11:35-42.

Stanier P, Abu-Hayyeh S, Murdoch JN, Eddleston J, Copp AJ (1998). Paralogous sm22alpha (Tagln). genes map to mouse chromosomes 1 and 9: further evidence for a paralogous relationship. Genomics 51:144-147.

Steward O, Schuman EM (2003). Compartmentalized synthesis and degradation of proteins in neurons. Neuron 40:347-59.

Strekalova T, Spanagel R, Bartsch D, Henn FA, Gass P (2004). Stress-induced anhedonia in mice is associated with deficits in forced swimming and exploration. Neuropsychopharmacology 29:2007-2017.

Sutcliffe JG (1988). mRNA in the mammalian central nervous system. Annu Rev Neurosci 11:157-198.

Swanson LW, Petrovich GD (1998). What is the amygdala? Trends Neurosci 21:323331.

Szumlinski KK, Dehoff MH, Kang SH, Frys KA, Lominac KD, Klugmann M et al. (2004). Homer proteins regulate sensitivity to cocaine. Neuron 43:401-413.

Szumlinski KK, Lominac KD, Kleschen MJ, Oleson EB, Dehoff MH, Schwarz MK et al. (2005). Behavioral and neurochemical phenotyping of Homer1 mutant mice: possible relevance to schizophrenia. Genes Brain Behav 4:273-288.

Takada D, Emi M, Ezura Y, Nobe Y, Kawamura K, Iino Y et al. (2002). Interaction between the LDL-receptor gene bearing a novel mutation and a variant in the apolipoprotein A-II promoter: molecular study in a 1135-member familial hypercholesterolemia kindred. J Hum Genet 47:656-664. 
Takahashi JS, Hong HK, Ko CH, McDearmon EL (2008). The genetics of mammalian circadian order and disorder: implications for physiology and disease. Nat Rev Genet 9:764-775.

Tannenbaum B, Anisman H (2003). Impact of chronic intermittent challenges in stressorsusceptible and resilient strains of mice. Biol Psychiatry 53:292-303.

Taylor BA (1989). Recombinant inbred strains. Genetic Variants and Strains of the Laboratory Mouse, 2nd edn. (Lyon ML, Searle AG, eds) Oxford University Press, Oxford.

Taylor BA, Wnek C, Kotlus BS, Roemer N, MacTaggart T, Phillips SJ (1999).

Genotyping new BXD recombinant inbred mouse strains and comparison of BXD and consensus maps. Mamm Genome 10:335-348.

Thomsen C, Klitgaard H, Sheardown M, Jackson HC, Eskesen K, Jacobsen P et al. (1994). (S)-4-carboxy-3-hydroxyphenylglycine, an antagonist of metabotropic glutamate receptor (mGluR). 1a and an agonist of $\mathrm{mGluR2}$, protects against audiogenic seizures in DBA/2 mice. J Neurochem 62:2492-2495.

Tolliver BK, Belknap JK, Woods WE, Carney JM (1994). Genetic analysis of sensitization and tolerance to cocaine. J Pharmacol Exp Ther 270:1230-1238.

Trinh JV, Nehrenberg DL, Jacobsen JR, Caron MG, Wetsel WC (2003). Differential psychostimulant-induced activation of neural circuits in dopamine transporter knockout and wild type mice. Neurosci 118:297-310.

Turri MG, Talbot CJ, Radcliffe RA, Wehner JM, Flint J (1999). High-resolution mapping of quantitative trait loci for emotionality in selected strains of mice. Mamm Genome 10:1098-1101.

Tursun B, Schlüter A, Peters MA, Viehweger B, Ostendorff HP, Soosairajah J et al. (2005). The ubiquitin ligase Rnf6 regulates local LIM kinase 1 levels in axonal growth cones. Genes Dev 19:2307-2319.

Ueda HR, Hayashi S, Chen W, Sano M, Machida M, Shigeyoshi Y et al. (2005). Systemlevel identification of transcriptional circuits underlying mammalian circadian clocks. Nat Genet 37:187-192.

Uher R, McGuffin P (2008). The moderation by the serotonin transporter gene of environmental adversity in the aetiology of mental illness: review and methodological analysis. Mol Psychiatry 13:131-146.

Valentinuzzi VS, Kolker DE, Vitaterna MH, Shimomura K, Whiteley A, Low-Zeddies S et al. (1998). Automated measurement of mouse freezing behavior and its use for quantitative trait locus analysis of contextual fear conditioning in (BALB/cJ C57BL/6J) F2 mice. Learn Mem 5:391-403.

Van de Kar LD, Piechowski RA, Rittenhouse PA, Gray TS (1991). Amygdaloid lesions: differential effect on conditioned stress and immobilization-induced increases in corticosterone and rennin secretion. Neuroendocrinology 54:89-95.

Venero C, Guadaño-Ferraz A, Herrero AI, Norström K, Manzano J, de Escobar GM et al. (2005). Anxiety, memory impairment, and locomotor dysfunction caused by a mutant 
thyroid hormone receptor $\alpha 1$ can be ameliorated by T3 treatment. Genes Dev 19:21522163.

Vermetten E, Schmahl C, Lindner S, Loewenstein RJ, Bremner JD (2006). Hippocampal and amygdalar volumes in dissociative identity disorder. Am J Psychiatry 163:630-636.

Vessey JP, Vaccani A, Xie Y, Dahm R, Karra D, Kiebler MA, Macchi P (2006).

Dendritic localization of the translational repressor Pumilio 2 and its contribution to dendritic stress granules. J Neurosci 26:6496-6508.

von Bartheld CS (2001). Comparison of 2-D and 3-D counting: the need for calibration and common sense. Trends Neurosci 24:504-506.

Vyas A, Bernal S, Chattarji S (2003). Effects of chronic stress on dendritic arborization in the central and extended amygdala. Brain Res 965:290-294.

Vyas A, Mitra R, Shankaranarayana Rao BS, Chattarji S (2002). Chronic stress induces contrasting patterns of dendritic remodeling in hippocampal and amygdaloid neurons. $\mathrm{J}$ Neurosci 22:6810-6818.

Wahlsten D, Bachmanov A, Finn DA, Crabbe JC (2006). Stability ofinbred mouse strain differences in behavior and brain size between laboratories and across decades. Proc Natl Acad Sci USA 103:16364-16369.

Wakana S, Sugaya E, Naramoto F, Yokote N, Maruyama C, Jin W et al. (2000). Gene mapping of SEZ group genes and determination of pentylenetetrazol susceptibility quantitative trait loci in the mouse chromosome. Brain Res 857:286-290.

Wallace RH, Wang DW, Singh R, Scheffer IE, George AL Jr, Phillips HA et al. (1998). Febrile seizures and generalized epilepsy associated with a mutation in the $\mathrm{Na}+$-channel beta1 subunit gene SCN1B. Nat Genet 19:366-370.

Wang X, Korstanje R, Higgins D, Paigen B (2004). Haplotype analysis in multiple crosses to identify a QTL gene. Genome Res 14:1767-1772.

Wehner JM, Radcliffe RA, Rosmann ST, Christensen SC, Rasmussen DL, Fulker DW et al. (1997). Quantitative trait locus analysis of contextual fear conditioning in mice. Nat Genet 17:331-334.

Weiss F, Ciccocioppo R, Parsons LH, Katner S, Liu X, Zorrilla EP et al. (2001).

Compulsive drug-seeking behavior and relapse. Neuroadaptation, stress, and conditioning factors. Ann N Y Acad Sci 937:1-26.

Wellman CL (2001). Dendritic reorganization in pyramidal neurons in medial prefrontal cortex after chronic corticosterone administration. J Neurobiol 49:245-253.

Wellman CL, Izquierdo A, Garret JE, Martin KP, Carroll J, Millstein R et al. (2007). Impaired stress-coping and fear extinction and abnormal corticolimbic morphology in serotonin transporter knock-out mice. J Neurosci 27:684-691.

Wiedholz LM, Owens WA, Horton RE, Feyder M, Karlsson RM, Hefner K et al. (2008). Mice lacking the AMPA GluR1 receptor exhibit striatal hyperdopaminergia and 'schizophrenia-related' behaviors. Mol Psychiatry 13:631-640. 
Wignall EL, Dickson JM, Vaughan P, Farrow TF, Wilkinson ID, Hunter MD et al. (2004). Smaller hippocampal volume in patients with recent-onset posttraumatic stress disorder. Biol Psychiatry 56:832-836.

Williams RW (2000). Mapping genes that modulate mouse brain development: a quantitative genetic approach. Mouse Brain Development. (Goffinet A, Rakic P, eds) Springer, Berlin.

Williams RW (2006). Expression genetics and the phenotype revolution. Mamm Genome 17:496-502.

Williams RW, Airey DC, Kulkarni A, Zhou G, Lu L (2001a). Genetic dissection of the olfactory bulbs of mice: QTLs on four chromosomes modulate bulb size. Behav Genet 31:61-77.

Williams RW, Bennett B, Lu L, Gu J, DeFries JC, Carosone-Link PJ et al. (2004). Genetic structure of the LXS panel of recombinant inbred mouse strains: a powerful resource for complex trait analysis. Mamm Genome 15:637-647.

Williams RW, Gu J, Qi S, Lu L (2001b). The genetic structure of recombinant inbred mice: high-resolution consensus maps for complex trait analysis. Genome Biol 2:RESEARCH0046.

Williams RW, Rakic P (1988). Three-dimensional counting: an accurate and direct method to estimate numbers of cells in sectioned material. J Comp Neurol 278:344-352.

Williams RW, Strom RC, Rice DS, Goldowitz D (1996). Genetic and environmental control of variation in retinal ganglion cell number in mice. J Neurosci 15:7193-7205.

Willis-Owen SA, Flint J (2007). Identifying the genetic determinants of emotionality in humans; insights from rodents. Neurosci Biobehav Rev 31:115-124.

Willner P, Moreau JL, Nielsen CK, Papp M, Sluzewska A (1996). Decreased hedonic responsiveness following chronic mild stress is not secondary to loss of body weight. Physiol Behav 60:129-134.

Wray GA, Hahn MW, Abouheif E, Balhoff JP, Pizer M et al. (2003). The evolution of transcriptional regulation in eukaryotes. Mol Biol Evol 20:1377-1419.

Yalcin B, Willis-Owen SA, Fullerton J, Meesaq A, Deacon RM, Rawlins JN et al. (2004). Genetic dissection of a behavioral quantitative trait locus shows that Rgs2 modulates anxiety in mice. Nat Genet 36:1197-11202.

Yang RJ, Mozhui K, Karlsson RM, Cameron HA, Williams RW, Holmes A (2008). Variation in mouse basolateral amygdala volume is associated with differences in stress reactivity and fear learning. Neuropsychopharmacology 33:2595-2604.

Yang X, Schadt EE, Wang S, Wang H, Arnold AP, Ingram-Drake L et al. (2006). Tissuespecific expression and regulation of sexually dimorphic genes in mice. Genome Res 16:995-1004.

Yehuda R (2002). Current status of cortisol findings in post-traumatic stress disorder. Psychiatr Clin North Am 25:341-368. 
Yehuda R, LeDoux J (2007). Response variation following trauma: a translational neuroscience approach to understanding PTSD. Neuron 56:19-32.

Yin S, Guan Z, Tang Y, Zhao J, Hong J, Zhang W (2005). Abnormal expression of epilepsy-related gene ERG1/NSF in the spontaneous recurrent seizure rats with spatial learning memory deficits induced by kainic acid. Brain Res 1053:195-202.

Zorawski M, Killcross S (2002). Posttraining glucocorticoid receptor agonist enhances memory in appetitive and aversive Pavlovian discrete-cue conditioning paradigms. Neurobiol Learn Mem 78:458-464.

Zubieta JK, Heitzeg MM, Smith YR, Bueller JA, Xu K, Xu Y et al. (2003). COMT val158met genotype affects mu-opioid neurotransmitter responses to a pain stressor. Science 299:1240-1243. 


\section{VITA}

Khyobeni Mozhui was born June 12, 1978, in Kohima, Nagaland. She belongs to an ethnic minority group of Tibetan-Burmese origins. She received her bachelor's degree in Biochemistry from Delhi University, India, in 2000. She then studied Life Sciences at the Jawaharlal Nehru University in Delhi, and received her master's degree in 2002. After her master's studies, she worked as a research assistant in the lab of Dr. B. N. Mallick where she studied the neural mechanism of REM sleep. In 2003, she joined the Neuroscience Program at the University of Tennessee Health Science Center and began her doctoral studies. The initial part of her research was done under the mentorship of the late Dr. David V. Smith. In 2006, she joined Dr. Robert Williams's lab to carry out her dissertation research. 